\title{
Subespaços e quocientes de $C(K)$ com poucos operadores
}

\author{
Alirio Gómez Gómez \\ TESE APRESENTADA \\ $\mathrm{AO}$ \\ Instituto DE MATEMÁticA E EstatísticA \\ DA \\ Universidade DE SÃo Paulo \\ PARA \\ OBTENÇÃO DO TÍTULO \\ $\mathrm{DE}$ \\ Doutor EM CiÊnCIAS
}

Programa: Matemática

Orientador: Prof. Dr. Rogério Augusto dos Santos Fajardo

Durante o desenvolvimento deste trabalho o autor recebeu auxílio financeiro da CAPES

São Paulo, Fevereiro de 2020 


\section{Título do trabalho a ser apresentado à CPG para a dissertação/tese}

Esta versão da tese contém as correções e alterações sugeridas pela Comissão Julgadora durante a defesa da versão original do trabalho, realizada em 20/01/2020. Uma cópia da versão original está disponível no Instituto de Matemática e Estatística da Universidade de São Paulo.

Comissão Julgadora:

- Prof. Dr. Rogério Augusto dos Santos Fajardo (orientador) - IME-USP

- Prof. Dr. Leonardo Pellegrini Rodrigues - IME-USP

- Prof. Dr. Leandro Cândido Batista - UNIFESP

- Prof. Dr. Samuel Gomes da Silva - UFBA

- Prof. Dr. Marcelo Dias PASSOS - UFBA 
Ao meu papai, José Martín (In memoriam), quem corrigiu os meus primeiros erros na matemática,

e despertou-me o amor pelo saber, À minha mamãe, Rosenda, que renunciou a todos os seus sonhos para que a minha formação fosse possível, Às minhas irmãs, Martha e Flor, pelo apoio incondicional, Ao meu sobrinho, Martín, pelas novas alegrias que trouxe à família. 


\section{Agradecimentos}

A meu orientador Prof. Dr. Rogério Augusto dos Santos Fajardo por todos os anos de dedicação como orientador, por sua paciência durante todo esse tempo e por sua cuidadosa supervisão do meu progresso em direção a esta tese, e pelos seus ensinamentos tanto na matemática quanto no português.

À Coordenação de Aperfeiçoamento de Pessoal de Nível Superior - Brasil (CAPES - Código de Financiamento 001) pelo apoio financeiro.

À Superintendência de Assistência Social - SAS da USP, pela bolsa moradia durante parte do período no qual não possuía bolsa.

A todos os professores do IME-USP que tive o prazer de conhecer. Especialemente, ao professor Cesar Polcino Milés pela oportunidade de vincular-me como monitor.

Agradeço também com especialidade aos professores, Alina Fedossova (UNAL - Bogotá) por sua confiança e apoio a longo destes anos desde a época prévia ao processo de admisão ao programa de doutorado, Gilberto Pérez (UPTC) por me apresentar à análise funcional, e Cesar Guauque por apoiar meus esforços em ir mais longe, durante o tempo de ensino fundamental.

À minha mamãe, Rosenda, e minhas irmãs Flor e Martha, por seu amor e apoio incondicional.

À minha namorada, Lara, por seu amor e dedicação. Também, por seu apoio e a sua paciência na etapa final desta jornada.

Aos meus amigos, Carlos Wilson Rodríguez, pelas inúmeras discussões sobre matemática, que me resultaram esclarecedoras, pelas conversas sobre outros tópicos fora da matemática que me permitiram aprender muito, e sobre tudo, por me fazer sentir parte de sua família; Leonardo Poveda por sua ajuda durante o processo de admissão ao programa de doutorado, e por assistir minhas apresentações preparatórias da qualificação e da defesa nas versões mais preliminares; Jackson Poveda, por sua recepção em minha chegada a São Paulo; Carito, por seu apoio e confidência nos dias difíceis. Também a os meus amigos: Sarita, Daniel, Adri, Bia (In memoriam), Edwin Pérez, Michael Rincón, Paola, Camilo Ángulo, Andrés Rosso, Wilson Cuellar, Iván, Cleber, Edwin Murcia, Lorena, Fernando, Jader, Laurita, Pablo, Raquel, Raibel, Hermeth, Verónica, e todos os que não nomeei aqui mas que fizeram mais leve esta jornada de meus estudos. 


\section{Resumo}

GÓMEZ-GÓMEZ, A. Subespaços e quocientes de $C(K)$ com poucos operadores. 2019. Tese (Doutorado) - Instituto de Matemática e Estatística, Universidade de São Paulo, São Paulo, 2019.

Um operador linear e contínuo $T: C(K) \longrightarrow C(K)$ é dito uma multiplicação fraca se é da forma $g I+S$, onde $g \in C(K), I$ é a identidade e $S$ é um operador fracamente compacto, e é dito multiplicador fraco se o operador adjunto $T^{*}$ em $C(K)^{*}$ é da forma $g I+S$, onde $g$ é uma função boreliana limitada e $S$ é fracamento compacto. O objetivo desta tese é estudar as propriedades de espaços de Banach da forma $C(K)$ em que todos os operadores são multiplicações fracas ou multiplicadores fracos, especialmente a respeito dos subespaços e quocientes desses espaços.

Neste trabalho são apresentadas algumas condições topológicas sobre $K$ relacionadas com a propriedade de $C(K)$ ter poucos operadores e é provada a existência de $C(K)$ indecomponível e contendo operadores que não são multiplicadores fracos. Assumindo Princípio $\diamond$, construímos $K$ contendo $\beta \mathbb{N}$ como subespaço e tal que $C(K)$ tem poucos operadores e contem $2^{\omega}$ quocientes indecomponíveis não isomorfos. Sob essa mesma hipótese conjuntística, apresentamos um exemplo de um espaço $C(K)$ contendo operadores não multiplicadores fracos e que $K$ não possui retrações não triviais. Mostramos, também, que $C(K)$ com poucos operadores não pode conter $c_{0}$ como quociente e, mais do que isso, se $c_{0}$ é quociente de $C(K)$, o conjunto dos operadores que não são multiplicadores fracos em $C(K)$, acrescidos do operador nulo, contém um subespaço de $\mathcal{L}(C(K))$ isomorfo a $l_{\infty}$.

Palavras-chave: Espaços fracamente Koszmider, multiplicador fraco, quociente indecomponível, espaçabilidade. 


\section{Abstract}

GÓMEZ-GÓMEZ, A. Subspaces and quotients of $C(K)$ spaces with few operators. 2019. Thesis (PhD) - Instituto de Matemática e Estatística, Universidade de São Paulo, São Paulo, 2019. A bounded linear operator $T: C(K) \longrightarrow C(K)$ is called a weak multiplication if there is $g \in C(K)$ such that $T=g I+S$, where $I$ is the identity operator and $S$ is a weakly compact operator, and it is called a weak multiplier if its adjoint operator $T^{*}$ in $C(K)^{*}$ can be written in the form $g I+S$, where $g$ is a bounded Borel function and $S$ is a weakly compact operator. The aim of this thesis is to study the Banach spaces $C(K)$ where all bounded linear operators are weak multiplications or weak multipliers, especially in relation to subspaces and quotients of those spaces.

In this work, some topological conditions on $K$, related to the property of $C(K)$ having few operators, are given, we also prove that there exists an indecomposable $C(K)$ that have some not weak multipliers operators. We assume axiom $\diamond$ and construct $K$ that contains an homeomophic copy of $\beta \mathbb{N}$ and $C(K)$ has few operators and it contains $2^{\omega}$ not isomorphic indecomposable quotients. Under the same set thoretical extra axiom we construct $C(K)$ that has some not weak multiplier operators and $K$ does not have non trivial retractions. Moreover, we prove that if $C(K)$ has few operators, it can not contain $c_{0}$ as a quotient, and furthermore, if $c_{0}$ is a quotient of $C(K)$, then the set of all not weak multiplier operators on $C(K)$, together with zero operator, contain a subspace of $\mathcal{L}(C(K))$ which is isomorphic to $l_{\infty}$.

Keywords: Weakly Koszmider space, weak multiplier, quotient indecomposable, spaceability. 


\section{Sumário}

$\begin{array}{ll}\text { Lista de Abreviaturas } & \text { ix }\end{array}$

Lista de Símbolos $\quad$ xi

$\begin{array}{ll}\text { Introdução } & 1\end{array}$

1 Preliminares $\quad 7$

1.1 Limites inversos de espaços topológicos . . . . . . . . . . . . . . . . . 7

1.2 Espaços de Banach da forma $C(K) \ldots \ldots \ldots \ldots$

1.3 Medidas de Radon e Representação de Riesz . . . . . . . . . . . . . . . . . . . . . . . . . . . . . . . .

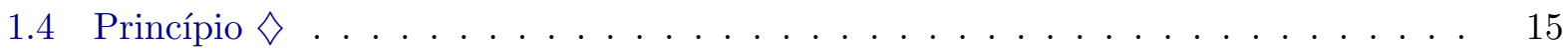

2 Espaços de Koszmider e fracamente Koszmider $\quad 17$

2.1 Operadores multiplicadores fracos e multiplicações fracas . . . . . . . . . . . . . . . . 17

2.2 Condições necessárias para $C(K)$ ter poucos operadores $\ldots \ldots \ldots$. . . . . . . . 22

2.3 Condições suficientes para $C(K)$ ter poucos operadores . . . . . . . . . . . . . 23

3 Subespaços complementados de espaços com poucos operadores 31

3.1 Espaços $C(K)$ indecomponíveis e espaços com poucos operadores . . . . . . . . . . 31

3.2 Subsespaços complementados de espaços $C(K)$ com $K$ fracamente Koszmider . . . 32

3.3 Espaços conexos fracamente Koszmider . . . . . . . . . . . . . . . . . . . 34

4 Conjunto dos Operadores Não Multiplicadores Fracos $\quad 37$

4.1 Lineabilidade e espaçabilidade do conjunto dos operadores não multiplicadores fracos de um espaço $C(K) \ldots \ldots \ldots \ldots \ldots \ldots$. . . . . . . . . . . . . . 37

4.2 Espaçabilidade dos operadores não multiplicadores fracos de $C(K)$ com $c_{0}$ como

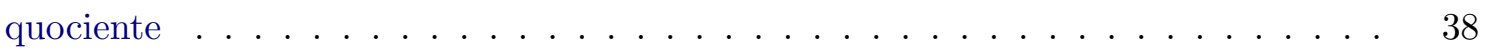

5 Extensões compactos por sequências de funções duas a duas disjuntas $\quad 43$

5.1 Extensão forte e extensão completa . . . . . . . . . . . . . . . . . 43

5.2 Construções indutivas usando extensões . . . . . . . . . . . . . . . . . . 45

6 Um espaço $C(K) \operatorname{com} 2^{\omega}$ quocientes indecomponíveis não isomorfos $\quad 67$

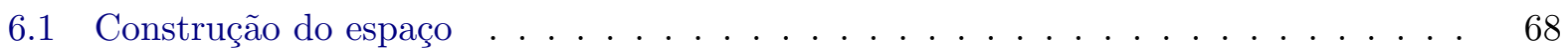

7 Um espaço não fracamente Koszmider e sem retrações não triviais $\quad \mathbf{7 7}$

7.1 Construção do espaço . . . . . . . . . . . . . . . . . . . . 77 
viii SUMÁRIO

8 Conclusões $\quad 87$

8.1 Comentários finais e problemas em aberto . . . . . . . . . . . . 89

$\begin{array}{ll}\text { Referências Bibliográficas } & 91\end{array}$

$\begin{array}{ll}\text { Índice Remissivo } & 94\end{array}$ 


\section{Lista de Abreviaturas}

$\mathrm{CH}$

$\operatorname{NWM}(C(K))$
Hipótese do continuum (continuum hypothesis)

Conjuntos dos operadores não multiplicadores fracos sobre $C(K)$

(Set of all operatos on $C(K)$ that are non weak multipliers) 


\section{Lista de Símbolos}

$\mathfrak{c}$

$\mathcal{L}(C(K))$

$\operatorname{supp}(f)$

$\lim _{\leftarrow}\left(X_{\alpha}, \pi_{\alpha, \beta}, I\right)$

$\mathcal{W}(X)$

$\approx$

$\mathcal{P}(\mathbb{N})$

$[\mathbb{N}]^{\omega}$

$\delta_{x}$
Cardinal do $\mathbb{R}$

Espaço de Banach conformado por todos os operadores lineares contínuous sobre $C(K)$.

Suporte da função $f$

Limite do sistema inverso $\left(X_{\alpha}, \pi_{\alpha, \beta}, I\right)$

Espaço dos operadores fracamente compactos em $X$

Isomorfismo entre espaços de Banach.

Conjunto das partes de $\mathbb{N}$

Coleção dos subconjuntos infinitos de $\mathbb{N}$

Medida de Dirac concentrada em $x$ 


\section{Introdução}

\section{Contexto histórico}

Uma das maneiras de entendermos a geometria de um espaço de Banach é analisando a diversidade de operadores lineares e limitados sobre este. A existência de classes de operadores específicos é garantia de algumas propriedades do espaço de Banach $X$. Por exemplo, a existência de projeções com imagem e núcleo de dimensão infinita implica que o espaço é decomponível, isto é, se decompõe como soma direta de dois subespaços de dimensão infinita. Nós dirigimos a nossa atenção neste trabalho para espaços que todos os seus operadores tem uma forma especifica. Doravante, sempre que nos referirmos a operadores sobre um espaço de Banach, referimos-nos aos operadores lineares e limitados.

Dentre as classes de operadores sobre um espaço de Banach merecem atenção aquelas formadas por operadores "pequenos" (no sentido da imagem produzida por esses operadores), como os operadores de imagem de dimensão finita, os operadores compactos, os fracamente compactos, os estritamente singulares (isto é, cuja restrição a um subespaço de dimensão infinita não é um isomorfismo sobre a imagem) e os de imagem separável (no contexto dos espaços de Banach não separáveis).

Naturalmente, espaços de dimensão infinita sempre possuem operadores que não são compactos, a saber, a identidade e seus múltiplos por escalares não nulos. A pergunta levantada por Lindestrauss em 1976 (veja [Lin75]), na lista de problemas em aberto nos espaços de Banach, conjeturava se existia algum espaço em que a identidade fosse essencialmente o único operador não compacto. Isto é, se existe um espaço de Banach onde todos os operadores sobre ele possam se escrever como a soma de um múltiplo escalar da identidade e um operador compacto.

Para exemplificar como o estudo dos operadores impacta nas propriedades de um espaço de Banach, é fácil verificar que projeçõs compactas possuem imagem de dimensão finita. Consequentemente, uma projeção da forma $c I+C$, onde $c$ é um escalar não nulo, $I$ é a identidade e $C$ um operador compacto, possui núcleo de dimensão finita. Dessa forma, em um espaço de Banach que responde afirmativamente a pergunta de Lindestrauss todo subespaço complementado ou possui dimensão finita ou codimensão finita. Um espaço dessa forma - que não se decompõe como soma direta de dois subespaços de dimensão infinita - é dito indecomponível.

Embora o problema postado por Lindestrauss só tenha sido resolvido afirmativamente em 2011, por Argyros e Haydon (veja [AH11]), avanços importantes feitos ao longo das décadas anteriores à aparição deste artigo deixaram intrigantes linhas de pesquisa. Procurou-se respostas parciais desse problema através de outras noções de operadores que são "pequenas perturbações da identidade". Isto é, espaços de Banach em que todos operadores são multiplicações por um escalar mais um operador "pequeno". 
O primeiro resultado nesse sentido, e inteiramente em ZFC, foi obtido em 1988, por Shelah e Steprāns, em [SS88]. Eles apresentaram um exemplo de um espaço de Banach $X_{S S}$ não separável em que todos os operadores $T$ são da forma

$$
T=\lambda I+R,
$$

onde $\lambda \in \mathbb{R}$ e $R$ é um operador com imagem separável. Shelah e Steprāns chamaram o espaço $X_{S S}$ de espaço com poucos operadores. Notamos que os operadores compactos possuem imagem separável, e nesse sentido o espaço $X_{S S}$ está relacionado ao problema de Lindestrauss.

Posteriormente, em 1993, Gowers e Mourey, em [GM93], apresentaram um exemplo de um espaço de Banach - usualmente denotado por $X_{G M}$ - no qual todos os operadores são da forma $T=\lambda I+S$, onde $\lambda \in \mathbb{R}$ e $S$ é um operador estritamente singular. No mesmo artigo, provaram, ainda, que, se $X$ é um espaço de Banach complexo Hereditariamente Indecomponível (i.e. Todo subespaço fechado é indecomponível, abreviado como H.I.), então todo operador $T$ de $X$ em $X$ pode ser escrito como $T=\lambda I+S$, onde $\lambda \in \mathbb{C}$ e $S$ é estritamente singular (ver Teorema 18, [GM93]).

Como corolário desse teorema, Gowers e Maurey deduziram que um espaço de Banach complexo H.I não é isomorfo a nenhum de seus subespaços próprios e, em particular, não é isomorfo a seus hiperplanos. Este último resultado é verdadeiro também no caso dos espaços de Banach reais e foi deduzido a partir do caso complexo. Mesmo assim para o espaço $X_{G M}$ que é real, em [GM93], foi provado diretamente que todos seus operadores lineares são da forma $\lambda I+S$.

Os espaços cujos operadores são uma perturbação de um múltiplo escalar da identidade por um operador estritamente singular, como em $X_{G M}$, ou por um operador compacto, como no espaço $X_{A H}$ construído por Argyros e Haydon, são chamados de espaços de Banach com poucos operadores. Usando esta terminologia, Gowers e Maurey em [GM93] abordaram três problemas clássicos dos espaços de Banach: (a) a existência de espaços que não tem subespaços complementados (não triviais), (b) espaços não isomorfos a seus hiperplanos, e (c) espaços com poucos operadores.

No contexto dos espaços Banach $C(K)$, o espaço das funções reais contínuas definidas sobre um espaço topológico compacto Hausdorff $K$, munido da norma do supremo, questões similares a (a)-(c) foram abordadas por Koszmider, em [Kos04]. Conforme argumento mostrado por ele, não é possível $C(K)$ ter poucos operadores no sentido de Gowers e Maurey (exibiremos a prova detalhada na Proposição 2.1). Tanto no caso $K$ conexo quanto no caso $K$ totalmente desconexo, assumindo a Hipótese do Contínuo, Koszmider construiu um espaço compacto Hausdorff $K$ tal que todo operador em $C(K)$ é da forma

$$
T=g I+S,
$$

onde $g \in C(K)$ e $S$ é estritamente singular. No mesmo artigo Koszmider construiu um exemplo em ZFC de um espaço topológico $K$ tal que todo operador $T$ sobre $C(K)$, o seu adjunto $T^{*}$ é da forma

$$
T^{*}=g I+S,
$$

onde $g: K \rightarrow \mathbb{R}$ é uma função de Borel limitada e $g I: C^{*}(K) \rightarrow C^{*}(K)$ é definido usando o Teorema de Representação de Riesz, por

$$
g I(\mu)(f)=\int g f d \mu
$$


onde $\mu$ pertence a $\mathcal{M}(K)$, o espaço das medidas de Radon sobre $K$. Os espaços compactos Hausdorff $K$ tais que todo operador em $C(K)$ são da forma (2) e (3) foram posteriormente chamados de espaços de Koszmider e espaços fracamente Koszmider respectivamente em [Sch08a].

Se $K$ é infinito, então $C(K)$ contém um subespaço isomorfo a $c_{0}$ e, portanto, não existem existem espaços hereditariamente indecomponíveis da forma $C(K)$. Mas a pergunta por espaços indecomponíveis, sim, tem sentido nesse contexto.

Como vimos, Koszmider, em [Kos04], mostrou que não é possível obter $C(K)$ com poucos operadores no sentido de Gowers e Maurey. Em particular, também não no sentido de Argyros e Haydon. Koszmider mostrou ainda que, para qualquer $K$ compacto Hausdorff e infinito, existe um operador $T: C(K) \rightarrow C(K)$ que não é da forma $T=g I+C$, onde $g \in C(K)$ e $C$ é um operador compacto. Por esse motivo o espaço dos operadores multiplicações fracas é, em algum sentido, o minimal possível no contexto dos espaços $C(K)$ 's. Justifica-se, assim, que os espaços $C(K)$, com $K$ Koszmider ou fracamente Koszmider, são chamados de espaços $C(K)$ com poucos operadores.

Como no caso do espaço construído em [GM93], $C(K)$ ter poucos operadores e, em particular, poucas projeções, tem consequências sobre a geometria do espaço concernente aos subespaços complementados. O espaço $K$ infinito compacto Hausdorff conexo construído por Koszmider tem a seguintes propriedades:

(A) Todo operador em $C(K)$ é multiplicação fraca (Koszmider assumiu CH, mas Plebanek, em [Ple04], deu um exemplo em ZFC);

(B) Todo operador em $C(K)$ é multiplicador fraco;

(C) $C(K)$ não é isomorfo a seus hiperplanos;

(D) $C(K)$ é indecomponível;

(E) Se $L$ é totalmente desconexo, $C(K)$ não é isomorfo a $C(L)$.

Também foi construído um espaço $K$ totalmente desconexo com as propriedades (A)-(C). Tanto no caso $K$ conexo quanto $K$ totalmente desconexo, para obter (A) foi assumido $C H$. Esta hipótese é retirada posteriormente por Plebanek, em [Ple04],

Como se relacionam as propriedades (A)-(E) tem se tornado objeto de pesquisa desde que [Kos04] foi publicado. O próprio Koszmider, em [Kos04], provou que (A) implica (B), e que se $C(K)$ verifica (B) então não é isomorfo a nenhum subespaço próprio, em particular, que (B) implica (C). Adicionalmente, demonstrou que, se $K \backslash F$ é conexo, para todo $F \subset K$ finito (incluindo vazio) então (B) implica (D). Posteriormente, Schlakow, em [Sch08a], provou que $K$ conexo verificando (A) implica que $K \backslash F$ é conexo, para todo $F$ finito. Juntando esses dois resultados, é possível concluir que (A) implica (D) sempre que $K$ é conexo. Uma prova direta deste fato pode ser encontrada em [Faj09]. Independentemente, Fajardo, em [Faj07], e Schlakow em [Sch08a], apresentaram exemplos de espaços que têm a propriedade (B) mas não (A). Avilés e Koszmider, em 2013, em [AK13], demonstraram que (E) não implica (C) nem (D) (e portanto também não (A) ou (B)). Schlakow, em [Sch08a], ainda, deu uma nova caraterização para os espaços fracamente Koszmider como sendo os espaços compactos Hausdorff $K$ tais que 
é fracamente compacto para todo $S, T \in \mathcal{L}(C(K))$.

Algumas perguntas sobre as relações entre (A)-(E) permanecem em aberto até hoje. Por exemplo, não se sabe se existe um espaço $C(K)$ indecomponível tal que $K$ não é fracamente Koszmider.

Como se mencionou antes, não existem espaços $C(K)$ hereditariamente indecomponíveis. Também não existem espaços $C(K)$ quociente indecomponíveis (i.e. todo espaço $C(K)$ tem um quociente decomponível), porque ou $C(K)$ contém $c_{0}$ complementado ou tem $\ell_{2}$ como quociente (ver, [Muj97]). No entanto, permanece aberta a possibilidade de achar espaços $C(K)$ tais que algumas propriedades (de A-E) sejam hereditárias com relação a uma classe extensa de quocientes. Koszmider já mencionou esta ideia no final de [Kos04].

Fajardo, em 2012, em [Faj12], assumindo o Princípio $\diamond$, construiu um espaço conexo, compacto Hausdorff tal que todo subespaço fechado $L$ de $K$ é fracamente Koszmider. Este espaço tem pelo menos $2^{\omega}$ quocientes indecomponíveis não isomorfos da forma $C(L)$, para $L \subset K$ (veja [Faj12]).

\section{Panorama geral da tese}

Nesta tese estudamos propriedades dos espaços fracamente Koszmider, abordando a questão da existência de espaços $C(K)$ que sejam hereditariamente indecomponíveis para uma grande classe de quocientes, e também estudamos o conjunto dos operadores não multiplicadores fracos.

No estudo dos espaços fracamente Koszmider, olhamos para a geometria do espaço $C(K)$, especificamente a relação com subespaços complementados, e também para as propriedades topológicas do espaço compacto Hausdorff $K$.

Estudamos as relações entre propriedades topológicas do espaço $K$ e a propriedade de que todos os operadores em $C(K)$ são multiplicadores fracos, continuando com o enfoque de [Sch08a] no estudo da rigidez do espaço $K$. Nesse artigo, Schlakow observou que, se $K$ é fracamente Koszmider e $K \backslash F$ conexo, para $F \subset K$ finito, então $K$ é topologicamente rígido, isto é, as únicas funções contínuas de $K$ em $K$ são as constantes e a identidade.

Provamos uma generalização desse resultado, mostrando que, para $K$ fracamente Koszmider, se existir uma função com o domínio como sendo o fecho de um aberto de $K$ e sua imagem contida num outro aberto disjunto do primeiro, então ela deve ser constante num aberto de $K$ contido no seu domínio. Isso permite provar que um espaço fracamente Koszmider sem pontos isolados não possui abertos homeomorfos.

Além disso, usamos a caraterização dos espaços fracamente Koszmider, dada por Schlakow, em [Sch08a], para provar que nem $C(K)$ nem algum dos seus subespaços complementados pode ser isomorfo a $X \oplus X$, para um espaço de Banach $X$ de dimensão infinita e $K$ fracamente Koszmider. Adicionalmente, seguindo a ideia do exemplo em [Sch08a] e resultados de [Faj10], provamos que existe um espaço $K$ fracamente Koszmider e conexo tal que $C(K)$ é decomponível. Ou seja, que a propriedade (B) não implica (D) acima, mesmo assumindo conexidade de $K$.

Em outra parte, seguimos as ideais de Koszmider, em [Kos04], e de Fajardo, em [Faj12], e assumimos o Princípio Diamante, para construir um exemplo de um espaço compacto $K$ que contém $\beta \mathbb{N}$ como subespaço e que $C(K)$ tem $2^{\omega}$ quocientes indecomponíveis não isomorfos. Este resultado se diferencia daquele provado em [Faj12] justamente porque o compacto construído por Fajardo não contem um subespaço homeomorfo a $\beta \mathbb{N}$ uma vez que subespaços fechados de $K$ são fracamente Koszmider e $C(\beta \mathbb{N}) \approx \ell_{\infty}$ que tem operadores não multiplicadores fracos. 
Adicionalmente, nós construímos, usando Princípio Diamante, um exemplo de um espaço $K$ não fracamente Koszmider que não possui retrações não triviais. Retrações em $K$ correspondem com subespaços complementados de $C(K)$ em virtude que cada retração $\varphi: K \rightarrow K$ define a projeção em $C(K)$ dada por $f \mapsto f \circ \varphi$. Assim este exemplo é uma resposta parcial à pergunta se a propriedade (D) acima implica (B).

Nossas construções usam sequências de funções com suportes contidos em abertos disjuntos no lugar de sequências de funções duas a duas disjuntas. Provamos que conjuntos enumeráveis de sequências de funções contínuas definidas num subespaço fechado de um espaço compacto Hausdorff, com suportes contidos em abertos disjuntos, podem ser estendidas continuamente ao espaço todo conservando a mesma propriedade para os suportes. Usando isso, estabelecemos condições suficientes para que uma classe de subespaços fechados seja constituída de espaços fracamente Koszmider.

Em [FKP13] (2013), Fajardo et. al. demonstraram que o conjunto dos operadores não multiplicadores fracos $\operatorname{NWM}(C(K))$ sobre um espaço $C(K)$ é vazio ou é espaçável (i.e. $\operatorname{NWM}(C(K)) \cup\{0\}$ contém um subespaço vetorial infinito dimensional fechado de $\mathcal{L}(C(K)))$. Continuando nesta linha de trabalho, provamos que, se $C(K)$ tem $c_{0}$ como quociente, o conjunto $\mathrm{NWM}(C(K))$ é c-espacável, isto é, $\operatorname{NWM}(C(K)) \cup\{0\}$ contém um subespaço vetorial de $\mathcal{L}(C(K))$ de densidade maior ou igual c. Para isso mostramos que $\operatorname{NWM}(C(K)) \cup\{0\}$ contém um subespaço de $\mathcal{L}(C(K))$ isomorfo a $\ell_{\infty}$. Essa linha de pesquisa mostra a dicotomia entre poucos operadores e muitos operadores em $C(K)$.

\section{Organização da tese}

A tese está dividida em sete capítulos. O Capítulo 1 dedica-se a resultados clássicos sobre Topologia, Análise Funcional e Topologia, não apresentando resultados originais.

No Capítulo 2 se prova que na definição de operadores multiplicadores fracos pode-se trocar sequências de funções duas a duas disjuntas por sequências de funções com suportes contidos em abertos disjuntos. Na Seção 2.3 provamos que é possível estender conjuntos enumeráveis destas funções preservando suportes contidos em abertos disjuntos (ver Lema 2.17) e, seguindo [Kos04, Faj12], são descritas condições suficientes para que um espaço compacto $K$ e um subespaço fechado sejam fracamente Koszmider. Nas Proposições 2.14 e 2.15 são apresentadas condições necessárias para que $K$ seja fracamente Koszmider.

No Capítulo 3 apresentamos alguns resultados sobre espaços isomorfos a subespaços complementados de espaços $C(K)$ com $K$ fracamente Koszmider (Teoremas 3.8 e 3.9). No Teorema 3.11 da Seção 3.3 mostramos um exemplo de um espaço fracamente Koszmider e não Koszmider $K$ com $C(K)$ indecomponível.

O Capítulo 4 foi dedicado ao estudo do conjunto dos operadores não multiplicadores fracos usando os conceitos de lineabilidade e espaçabilidade. Os conceitos e resultados encontrados na literatura são apresentados na Seção 4.1, e a prova do teorema principal do capítulo é feita na segunda seção.

No Capítulo 5 são estudadas as extensões de compactos por sequências de funções contínuas, duas a duas disjuntas. Seguindo ideias de [Faj12], demonstramos que é possível fazer extensões de um compacto que preservam um homeomorfismo entre dois fechados disjuntos que são fechos de abertos e ao mesmo tempo que verificam propriedades similares as obtidas por Fajardo em 
[Faj12]. A maioria dos resultados deste capítulo são usados nas construções dos Capítulos 6 e 7 onde são construídos um espaço compacto $K$ fracamente Koszmider que contem um subespaço homeomorfo a $\beta \mathbb{N}$ e $C(K)$ tem $2^{\omega}$ quocientes indecomponíveis não isomorfos, e um espaço compacto não fracamente Koszmider que não contém retrações não triviais, respetivamente.

Os principais resultados do Capítulo 3 e uma versão mais fraca do Teorema 4.5 do Capítulo 4 foram publicados em conjunto com Fajardo e Pellegrini, em 2019, em [FGP19].

\section{Terminologia geral e notação}

- Os operadores $T: X \rightarrow Y$ entre espaços de Banach $X$ e $Y$ sempre serão considerados lineares e contínuos. A norma de um operador é a usual, dada por $\|T\|=\sup \{\|T x\|:\|x\| \leq 1, x \in X\}$.

- O espaço dos operadores lineares e contínuos de $X$ em $Y$ é denotado por $\mathcal{L}(X, Y)$. No caso $X=Y$, escrevemos apenas $\mathcal{L}(X)$.

- Assumimos que todos os espaços topológicos compactos nesta tese são infinitos e Hausdorff, mesmo quando não explicitado.

- Para um espaço topológico compacto $K$, denotamos por $C(K)$ o espaço das funções contínuas reais definidas sobre $K$ munido da norma do supremo.

- Se $K$ é espaço topológico compacto, $M(K)$ denota o espaço das medidas de Radon (com sinal) sobre $K$.

- Um subconjunto $M$ de um espaço topológico $X$ é dito raro em $X$ se o fecho de $M$ em $X$ tem interior vazio. $\mathrm{Ou}$, equivalentemente, se para todo aberto não vazio $U$ de $K$ existe $V \subset U$ aberto (em $K$ ) não vazio e disjunto de $M$.

- Se $M$ é um subconjunto de um espaço topológico $X$, denotamos por $\bar{M}$ o fecho de $M$ em $X$. Quando houver risco de ambiguidade, eventualmente explicitamos em qual espaço o fecho está sendo tomado.

- Se $f$ é uma função de um espaço topológico $X$ em $\mathbb{R}$, definimos o suporte de $f$, que denotamos por $\operatorname{supp}(f)$, como o conjunto $\overline{\{x \in X: f(x) \neq 0\}}$. 


\section{Capítulo 1}

\section{Preliminares}

Este capítulo está dividido em três seções, a primeira dedicada ao limite inverso de espaços topológicos focando principalmente em subespaços compactos de produtos infinitos do intervalo $[0,1]$. Na Seção 1.2 apresentamos resultados bem conhecidos sobre os espaços $C(K)$, especialmente relacionados com subespaços complementados e operadores fracamente compactos. A Seção 1.3 foi dedicada às medidas de Radon. Vários resultados aqui apresentados são cálculos rotineiros e serão usados no Capítulo 6.

\subsection{Limites inversos de espaços topológicos}

Lembramos que um sistema inverso de espaços topológicos é uma família $\left(X_{\alpha}, \pi_{\alpha, \beta}, I\right)$, onde $I$ é um conjunto dirigido por uma relação de ordem $\leq, X_{\alpha}$ é um espaço topológico, para cada $\alpha \in I$, tal que, para todo $\alpha, \beta \in I$, com $\alpha \leq \beta, \pi_{\alpha, \beta}$ é uma função contínua sobrejetora de $X_{\beta}$ em $X_{\alpha}$. O limite de um sistema inverso é o espaço topológico definido como:

$$
\lim _{\leftarrow}\left(X_{\alpha}, \pi_{\alpha, \beta}, I\right):=\left\{x \in \prod_{\alpha \in I} X_{\alpha}: \pi_{\beta}(x)=\pi_{\beta, \gamma}\left(\pi_{\gamma}(x)\right) \text { sempre que } \beta \leq \gamma, \text { e } \beta, \gamma \in I\right\}
$$

onde, para $\beta \in I, \pi_{\beta}$ é a projeção canônica de $\prod_{\alpha \in I} X_{\alpha}$ sobre $X_{\beta}$. A topologia é definida como a topologia do subespaço do espaço produto.

Se $X=\lim _{\leftarrow}\left(X_{\alpha}, \pi_{\alpha, \beta}, I\right)$, a restrição

$$
\left.\pi_{\beta}\right|_{X}: X \rightarrow X_{\beta}
$$

é sobrejetora e é dita da projeção canônica de $X$ sobre $X_{\beta}$. Usaremos a mesma notação $\pi_{\beta}$ tanto para a projeção canônica do espaço produto quanto para a do limite inverso em $X_{\beta}$. Além disso, escreveremos $\lim _{\leftarrow} X_{\alpha}$ no lugar de $\lim \left(X_{\alpha}, \pi_{\alpha, \beta}, I\right)$ quando $\pi_{\alpha, \beta}$ estiver claro no contexto (em especial, quando for a restrição da projeção canônica de $[0,1]^{\beta}$ em $[0,1]^{\alpha}$, para $\alpha \leq \beta$ ordinais).

Proposição 1.1 (Proposition 2.2.5, [Eng89]). Sejam I um conjunto dirigido e J um subconjunto cofinal em I. Sejam $\left(X_{\alpha}, \pi_{\alpha, \beta}, I\right)$ um sistema inverso de espaços topológicos indexado por $I, X=$ $\lim _{\leftarrow} X_{\alpha}$ e $\pi_{\alpha}: X \rightarrow X_{\alpha}$ a aplicação canônica. Então a familia de todos os conjuntos da forma $\pi_{\alpha}^{-1}[U]$, para $\alpha \in J$ e $U$ aberto em $X_{\alpha}$, é uma base para a topologia $X$. Além disso, se para cada $\alpha$ temos fixada uma base $\mathcal{B}_{\alpha}$, então a subfamília formada pelos conjuntos da forma $\pi_{\alpha}^{-1}[U]$, para 
$U \in \mathcal{B}_{\alpha}$ e $\alpha \in J$, é ainda uma base de $X$.

A partir de agora, sempre que mencionarmos ao limite de um sistema inverso, sem indicar qual a base topológica, nos referimos à base estabelecida na proposição anterior. Lembramos também que o limite se um sistema inverso de espaços topológicos Hausdorff é fechado no espaço produto desses espaços. Mais do que isso, o limite inverso preserva os axiomas de separação $T_{i}$, para $i \leq 3 / 2$. Para os detalhes sobre estas propriedades indicamos [Eng89].

Agora, seja $\lambda$ um ordinal limite. Consideremos o sistema inverso $\left(K_{\alpha}, \pi_{\alpha, \beta}, \lambda\right)$ onde $K_{\alpha} \subset[0,1]^{\alpha}$ é compacto e tal que, se $\beta>\alpha$ e $t \in K_{\beta}$, então $\left.t\right|_{\alpha} \in K_{\alpha}$; e as funções contínuas $\pi_{\alpha, \beta}: K_{\beta} \rightarrow K_{\alpha}$ para $\beta \geq \alpha$, definidas pela restrição $\left.t \mapsto t\right|_{\alpha}$.

O subespaço

$$
K_{\lambda}:=\left\{x \in[0,1]^{\lambda}:\left.x\right|_{\alpha} \in K_{\alpha}, \alpha<\lambda\right\}
$$

de $[0,1]^{\lambda}$ é homeomorfo ao $\lim K_{\alpha}$ pela aplicação $x \mapsto\left(\left.x\right|_{\alpha}\right)_{\alpha<\lambda}$, que é a restrição ao $K_{\lambda}$ do homeomorfismo natural $\varphi$ de $[0,1]^{\lambda}$ sobre $\prod_{\alpha<\lambda}[0,1]^{\alpha}$. Em consequência, para frente nos referiremos a $K_{\lambda}$ como o limite do sistema inverso. Assim, tendo em conta a Proposição 1.1, uma base de abertos para $K_{\lambda}=\lim _{\leftarrow} K_{\alpha}$ é uma família dos subconjuntos de $K_{\lambda}$ da forma $K_{\lambda} \cap \prod_{\alpha<\lambda} V$ onde $V$ é um intervalo aberto em $[0,1]$ com extremos racionais e $V_{\alpha} \neq[0,1]$ apenas para um número finito de $\alpha^{\prime}$ s.

A próxima proposição será usada no Capítulo 5.

Proposição 1.2. Sejam $K$ e $K^{\prime}$ espaços compactos e $f: K \rightarrow K^{\prime}$ uma função contínua e sobrejetora. Se $U \subset K$ é aberto tal que $f^{-1}[f[U]]=U$, então $f[U]$ é aberto em $K^{\prime}$

Demonstração: Sejam $U$ e $f$ como no enunciado da proposição. Como $K$ e $K^{\prime}$ são compactos e Hausdorff, então a continuidade de $f$ implica que $f[K \backslash U]$ é compacto e, portanto, fechado em $K^{\prime}$. Assim, para completar a demonstração é suficiente provar que $f[K \backslash U]=K^{\prime} \backslash f[U]$.

Se $s \in f[K \backslash U]$, então existe $t \in K$ tal que $t \notin U$ e $f(t)=s$. Como $f^{-1}[f[U]]=U$, então $f(t) \notin U$ e, portanto, $s=f(t) \in K^{\prime} \backslash f[U]$, provando que $f[K \backslash U] \subset K^{\prime} \backslash f[U]$.

Por outro lado, se $s \in K^{\prime} \backslash f[U]$, então $f^{-1}[\{s\}] \cap U=\emptyset$, pois $f^{-1}[f[U]]=U$. Logo, $s \in f[K \backslash U]$ e, portanto, $K^{\prime} \backslash f[U] \subset f[K \backslash U]$.

As uniões de abertos básicos no limite de um sistema inverso de espaços topológicos compactos Hausdorff indexado por um ordinal com cardinalidade $2^{\omega}$ tem imagem aberta por alguma projeção canônica. Lembramos que uma função contínua e sobrejetora entre espaços metrizáveis (mesmo subconjuntos de $\mathbb{R}$ ) não é, em geral, aberta.

Proposição 1.3. Sejam $X=\lim _{\leftarrow}\left(X_{\alpha}, \pi_{\alpha \beta}, I\right)$ e $\alpha<\beta$. Se $U_{\alpha}$ é um aberto em $K_{\alpha}$, então

$$
\pi_{\alpha, \beta}^{-1}\left[U_{\alpha}\right]=\pi_{\beta}\left[\pi_{\alpha}^{-1}\left[U_{\alpha}\right]\right]
$$

Em consequência, $\pi_{\beta}^{-1}\left[\pi_{\alpha, \beta}^{-1}\left[U_{\alpha}\right]\right]=\pi_{\alpha}^{-1}\left[U_{\alpha}\right]$ e, se I é um cardinal $\kappa$ com cofinalidade não enumerável, então para qualquer união enumerável $U$ de abertos básicos de $X$ existe $\gamma<\kappa$ tal que, para todo $\alpha>\gamma$, ocorre $\pi_{\alpha}^{-1}\left[\pi_{\alpha}[U]\right]=U$ e, portanto, se $X$ e $X_{\alpha}$ são compactos, para todo $\alpha<\kappa$, $\pi_{\alpha}[U]$ é aberto em $X_{\alpha}$ para $\alpha>\gamma$. 
Demonstração: Se $\alpha<\beta, U_{\alpha}$ é um aberto em $X_{\alpha}$ e $x \in \pi_{\alpha}^{-1}\left[U_{\alpha}\right]$, então $\pi_{\alpha, \beta}\left(\pi_{\beta}(x)\right) \in U_{\alpha}$. Portanto, se $y=\pi_{\beta}(x)$, então $y \in \pi_{\alpha, \beta}^{-1}\left[U_{\alpha}\right]$, i.e. $\pi_{\beta}\left[\pi_{\alpha}^{-1}\left[U_{\alpha}\right]\right] \subset \pi_{\alpha, \beta}^{-1}\left[U_{\alpha}\right]$.

Por outro lado, se $y \in \pi_{\alpha, \beta}^{-1}\left[U_{\alpha}\right]$, pela sobrejetividade das aplicações $\pi_{\alpha}$, existe $x \in X$ tal que $\pi_{\beta}(x)=y$. Logo, $x \in \pi_{\alpha}\left[U_{\alpha}\right]$ e $\pi_{\alpha, \beta}\left(\pi_{\alpha}(x)\right)=\pi_{\alpha, \beta}(y)$. Portanto, $x \in \pi_{\alpha}^{-1}\left[\left\{\pi_{\alpha, \beta}(y)\right\}\right] \subset \pi_{\alpha}^{-1}\left[U_{\alpha}\right]$, ou seja $y \in \pi_{\beta}\left[\pi_{\alpha}^{-1}\left[U_{\alpha}\right]\right]$. Assim provamos a primeira parte da proposição.

Para achar $\gamma$ satisfazendo a segunda parte da proposição anterior, usamos a hipótese sobre a cofinalidade de $\kappa$. Como $U=\bigcup_{n \in \mathbb{N}} \pi_{\alpha_{n}}^{-1}\left[U_{\alpha_{n}}\right]$, onde $\alpha_{n}<\kappa$, para todo $n \in \mathbb{N}$, então existe $\gamma=$ $\sup \left\{\alpha_{n}: n \in \mathbb{N}\right\}<\kappa$. Pela primeira parte da observação $\pi_{\alpha_{n}, \alpha}^{-1}\left[U_{\alpha_{n}}\right]=\pi_{\alpha}\left[\pi_{\alpha_{n}}^{-1}\left[U_{\alpha_{n}}\right]\right]$, para todo $n \in \mathbb{N}$ e todo $\alpha>\gamma$. Logo, $\pi_{\alpha}^{-1}\left[\pi_{\alpha}[U]\right]=U$ segue da propriedade distributiva de uniões e intersecções de conjuntos e imagens inversas de funções.

Segue imediatamente da Proposição 1.2 que o conjunto $\pi_{\alpha}[U]$ é aberto.

Vale destacar que a segunda parte da proposição anterior é válida, para $\kappa=\omega_{1}$ e para $\kappa=2^{\omega}$. No primeiro caso segue da regularidade de $\omega_{1}$, e no segundo do Lema de König (ver [Jec03] ou [Kun80]).

No limite de um sistema inverso $\lim \left(K_{\alpha}, \pi_{\alpha, \beta}, 2^{\omega}\right)$ os fechos dos abertos são imagens inversas por alguma projeção do fecho de um aberto. Estabelecemos este fato na seguinte observação.

Observação 1. Sejam $K_{\alpha} \subset[0,1]^{\alpha}$ compactos, para $\alpha<\omega_{1}, K$ o limite inverso dos $K_{\alpha}$ definido como antes, $\pi_{\alpha}: K \rightarrow K_{\alpha}$ a projeção canônica, e $U$ um aberto em $K$. Se $\beta<\omega_{1}$ é tal que $\pi_{\beta}^{-1}\left[\pi_{\beta}[U]\right]=U$ e $\left.z\right|_{\beta} \in \overline{\pi_{\beta}[U]}$ ent $\tilde{a} o z \in \bar{U}$

Para demonstrar a observação raciocinamos pela contrapositiva. Supondo $z \notin \bar{U}$, existe $V$ aberto em $K$ contendo $z$ e disjunto de $U$. Podemos supor $V$ um aberto elementar, isto é, $V=$ $\prod_{\xi<\omega_{1}} I_{\xi} \cap K$, onde $I_{\xi}$ é um intervalo aberto de extremos racionais e $I_{\xi} \neq[0,1]$ apenas para um número finito de $\xi$ 's.

Seja $V^{\prime}:=\prod_{\xi<\omega_{1}} J_{\xi} \cap K$, onde $J_{\xi}=I_{\xi}$ para $\xi<\beta$ e $J_{\xi}=[0,1]$ para $\xi \geq \beta$. Note que $z \in V \subset V^{\prime}$ e $V^{\prime} \cap U=\emptyset$. De fato, suponhamos, por absurdo, que existe $x \in V^{\prime} \cap U$. Como $\pi_{\beta}[V]=\pi_{\beta}\left[V^{\prime}\right]$, existe $y \in V$ tal que $\left.x\right|_{\beta}=\left.y\right|_{\beta}$ e, portanto, $x \in U$ e $y \notin U$, pois $V \cap U=\emptyset$, contradizendo que $\pi_{\beta}^{-1}\left[\pi_{\beta}[U]\right]=U$.

Como $\pi_{\beta}^{-1}\left[\prod_{\xi<\beta} J_{\xi} \cap K_{\beta}\right]=\prod_{\xi<\omega_{1}} J_{\xi} \cap K$, então $U \cap V^{\prime}=\emptyset$ implica $\prod_{\xi<\beta} J_{\xi} \cap K_{\beta} \cap \pi_{\beta}[U]=\emptyset$. Logo $\left.z\right|_{\beta} \notin \overline{\pi_{\beta}[U]}$, provando a observação.

Ressaltamos que em geral não é verdade que para um aberto $U$ em $[0,1]^{\omega_{1}}$ se verifique $\pi_{\beta}[U] \cap$ $K_{\beta}=\pi_{\beta}[U \cap K]$, mesmo quando $\pi_{\beta}^{-1}\left[\pi_{\beta}[U \cap K]\right]=U \cap K$. Além disso também não acontece em geral que $\pi_{\beta}^{-1}\left[\pi_{\beta}[U] \cap K_{\beta}\right]=U \cap K_{\beta}$.

\subsection{Espaços de Banach da forma $C(K)$}

Lembramos que um subespaço $Y$ de um espaço de Banach $X$ é dito complementado em $X$ se existe um operador $P: X \rightarrow X$, chamado de projeção, tal que $\left.P\right|_{Y} \equiv I d_{Y}$. Isto é equivalente a $P^{2}=P \operatorname{com} P(X)=Y$, ou à existência de um espaço $Z$ tal $X=Y \oplus Z$. Se $P$ é uma projeção sobre $Y$ então $\operatorname{Ker}(P)=Z$. 
Um espaço de Banach $X$ é indecomponível se não contém subespaços complementados de dimensão e codimensão infinita. No caso contrário, se $X=Y \oplus Z$ e $\operatorname{dim} Y$ ou $\operatorname{dim} Z$ é finita então se diz que $Y \oplus Z$ é uma decomposição trivial de $X$.

O Lema de Riesz para existência de vetores quase perpendiculares - que afirma que se $Y$ é um subespaço fechado próprio de um espaço de Banach $X$, então para todo $0<\varepsilon<1$ existe $x \in X$ com $\|x\|=1$ tal que $\|y-x\|>1-\varepsilon$ para todo $y \in Y$ - permite provar que toda projeção possui imagem de dimensão finita. Portanto a decomposição do espaço nesse caso é trivial.

Proposição 1.4. Sejam $X$ um espaço de Banach e $P \in \mathcal{L}(X)$ uma projeção. Se $P$ é compacto então $P(X)$ tem dimensão finita.

Existem varios exemplos espaços decomponíveis. Por exemplo, pelo Teorema de Sobczyk, todos espaços de Banach separáveis que contêm $c_{0}$ são decomponíveis.

Teorema 1.5 (Sobczyk). Sejam $X$ um espaço de Banach separável e $Y$ um subespaço de $X$ isomorfo a $c_{0}$, então $Y$ é complementado em $X$. Além disso, se $Y$ é isométrico a $c_{0}$, então existe uma projeção de $X$ sobre $Y$ com norma menor ou igual a 2.

No caso dos espaços de Banach $C(K)$ isto se traduz em que $C(K)$ contém $c_{0}$ complementado sempre que $K$ é compacto metrizável, pelo seguinte resultado clássico

Teorema 1.6. Se $K$ é compacto, então $C(K)$ é separável se, e somente se, $K$ é metrizável.

O próximo resultado (clássico) assegura que existem espaços $C(K)$ não separáveis que contêm $c_{0}$ complementado. A projeção usada na prova será referida mais para frente.

Proposição 1.7. Se K é um espaço compacto que contém uma sequência convergente não trivial, então $C(K)$ contém $c_{0}$ complementado.

Demonstração: Seja $x_{n} \rightarrow x$, onde todos os $x_{n}$ 's são diferentes. Como $K$ é Hausdorff, é possível construir uma sequência $\left(U_{n}\right)_{n \in \mathbb{N}}$ de abertos dois a dois disjuntos em $K$ tais que $x_{n} \in U_{n}$ e $x \notin U_{n}$, para todo $n \in \mathbb{N}$. Usando o Lema de Urysohn, construímos funções contínuas $e_{n}: K \rightarrow[0,1]$ tais que $e_{n}\left(x_{n}\right)=1$ e $e_{n}(z)=0$ se $z \in K \backslash U_{n}$.

Definimos o operador $T: c_{0} \rightarrow C(K)$ por

$$
T(s)=\sum_{n \in \mathbb{N}} s(n) e_{n}
$$

É fácil verificar que $T$ é linear. Como $U_{n} \cap U_{m}=\emptyset$, se $m \neq n$, então $T$ é injetor e, como $\|T(s)\|=$ $\sup |s(n)|$, então $T$ é uma imersão isométrica de $c_{0}$ em $C(K)$.

Agora, note que $\left(f\left(x_{n}\right)-f(x)\right)_{n \in \mathbb{N}} \in c_{0}$, para todo $f \in C(K)$, e, portanto, o operador $P$ : $C(K) \rightarrow T\left[c_{0}\right]$ definido por

$$
P(f)=\sum_{n \in \mathbb{N}}\left(f\left(x_{n}\right)-f(x)\right) e_{n}
$$

é contínuo. Mais ainda, como $P(T(s))=T(s)$ então $P$ é uma projeção de $C(K)$ sobre $T\left[c_{0}\right]$.

O espaço dos operadores lineares entre um espaço $C(K)$ e um espaço que não contém $c_{0}$ como subespaço se reduz a apenas os operadores fracamente compactos. 
Teorema 1.8 (Pełczyński, Teorema 5, [Peł60]). Sejam K um espaço compacto e $X$ um espaço de Banach tal que nenhum subespaço é isomorfo a c $c_{0}$. Então todo operador linear $T$ de $C(K)$ em $X$ é fracamente compacto.

Assim, por exemplo, se $K$ é compacto, todos os operadores de $C(K)$ em $\ell_{1}$ - o espaço das sequências reais absolutamente somáveis - são fracamente compactos.

Se, além disso, o espaço $K$ é metrizável, então uma condição necessária e suficiente para que os operadores de $C(K)$ em um espaço de Banach $X$ fixado sejam fracamente compactos é $X$ não conter copias isomorfas a $c_{0}$.

Corolário 1.9 (Corolário 1, [Peł60]). Sejam X um espaço de Banach e $K$ um espaço compacto metrizável. São equivalentes:

(i) Todo operador de $C(K)$ em $X$ é fracamente compacto.

(ii) X não contém subespaço isomorfo a $c_{0}$.

Por [Peł60], no corolário anterior, se o espaço $X$ é, em particular, um subespaço complementado de $C(K)$, com $P$ a projeção de $C(K)$ sobre $X$, e $X$ não contém cópia de $c_{0}$, então $P$ é fracamente compacto. Mais ainda, $P$ seria compacto pelo Teorema de Dunford-Pettis (ver por exemplo [DS58], p. 494.), que diz que o quadrado de todo operador fracamente compacto de $C(K)$ em $C(K)$ é compacto. Mas como se mencionou antes as projeções compactas tem dimensão finita. Assim:

Corolário 1.10 (Corolário 2, [Peł60]). Sejam K um espaço topológico compacto Hausdorff e X um subespaço complementado de $C(K)$. Então ou $X$ contém um subespaço isomorfo a $c_{0}$ ou $X$ é de dimensão finita.

Como $\ell_{1}$ não contém cópia de $c_{0}$, é imediato do corolário anterior que $\ell_{1}=c_{0}^{*}$ não pode ser subespaço complementado (com complementar de dimensão infinita) de $C(K)$. Esta conclusão também pode ser obtida do fato de $\ell_{1}=c_{0}^{*}$ e $c_{0}$ não conter subespaços isomorfos ao $\ell_{\infty}$, como se indica no próximo corolário.

Corolário 1.11 (Corolário 3, [Peł60]). Seja X um subespaço complementado de $C(K)$. Então, se $X$ é isomorfo ao espaço conjugado $Y^{*}$ de um espaço de Banach $Y, X$ contém um subespaço isomorfo a $l_{\infty}$ ou $X$ tem dimensão finita.

O próximo lema é um resultado combinatório que permite demonstrar vários teoremas clássicos sobre os operadores fracamente compactos. Por exemplo, na monografia de [DU77], por Diestel e Ulh, muitos resultados da teoria de medidas vetoriais é obtida usando este resultado. Nós o usaremos no Capítulo 2 e de maneira implícita no Capítulo 5. Aqui nos limitamos ao caso enumerável, que é suficiente para nosso trabalho.

Lema 1.12 (Lema de Rosenthal). Sejam $\Sigma$ uma $\sigma$-álgebra de subconjuntos de um conjunto $\Omega$ $e\left(\mu_{n}\right)_{n \in \mathbb{N}}$ uma sequência uniformemente limitada de medidas não negativas, finitamente aditivas definidas em $\Sigma$. Então, para toda sequência duas a duas disjunta $\left(E_{n}\right)_{n \in \mathbb{N}}$ em $\Sigma$ e todo $\varepsilon>0$, existe uma sequência estritamente crescente $\left(n_{k}\right)_{k \in \mathbb{N}}$ de números naturais tal que

$$
\mu_{n_{k}}\left(\bigcup_{j \neq k} E_{n_{j}}\right)<\varepsilon \text { para cada } k \in \mathbb{N} .
$$


O Teorema 1.8 acima pode ser deduzido imediatamente do próximo teorema, cuja prova é baseada no Lema de Rosenthal, ver [DU77].

Teorema 1.13 (Teorema 15, VI, [DU77]). Sejam $K$ compacto e $X$ um espaço de Banach. Se $T: C(K) \rightarrow X$ não é fracamente compacto, então $C(K)$ contém um subespaço $E$ isometricamente isomorfo a c col que $\left.T\right|_{E}$ é um isomorfismo.

Note que o Teorema 1.13 estabelece que, se $T: C(K) \rightarrow X$ é um operador estritamente singular, então $T$ é fracamente compacto. A recíproca desta afirmação é verdadeira, e segue do Teorema de Dunford-Pettis. Para ver os detalhes indicamos [AK06] p. 118-119.

Teorema 1.14 (Pełczyński). Sejam $K$ um espaço compacto e $X$ um espaço de Banach. Um operador linear $T: C(K) \rightarrow X$ é fracamente compacto se e somente se é estritamente singular.

Outra consequência do Teorema 1.13 é uma caraterização dos operadores fracamente compactos em $C(K)$ como sendo os que transformam sequências (de funções contínuas) duas a duas disjuntas (i.e. $f_{n} \cdot f_{m}=0$ sempre que $m \neq n$ ) em sequências que convergem a zero em norma.

Teorema 1.15 ([DU77], VI, p. 160). Sejam K compacto e $X$ um espaço de Banach. As seguintes afirmações sobre um operador $T: C(K) \rightarrow X$ são equivalentes:

(a) $T$ é fracamente compacto

(b) T transforma sequências fracamente de Cauchy em sequências convergentes na norma.

(c) Se $\left(f_{n}\right)_{n \in \mathbb{N}}$ é um sequência em $C(K) \operatorname{com} f_{n} \cdot f_{m}=0$ para $m \neq n$, então $\lim _{n \rightarrow \infty} T\left(f_{n}\right)=0$.

Por (c) do teorema anterior, a projeção construída na prova da Proposição 1.7 não é fracamente compacta. É possível afirmar algo mais forte:

Corolário 1.16. Se $K$ é um espaço compacto que contém sequências convergentes não trívias, então existe um operador em $\mathcal{L}(C(K))$ que não é da forma $g I+S$ onde $g \in C(K)$, I é o operador identidade, e $S$ é fracamente compacto.

Demonstração: Seja $x_{n} \rightarrow x$ em $K$ sem repetições. Fixe uma sequência de funções contínuas $e_{n}$ de $K$ em [0,1], duas a duas disjuntas com $e_{n}(x)=0$ e $e_{n}\left(x_{n}\right)=1$, para todo $n \in \mathbb{N}$, e considere $T$ a imersão isométrica de $c_{0}$ em $\left.C(K) \operatorname{com} T\left(\left(a_{n}\right)_{n \in \mathbb{N}}\right)\right)=\sum_{n \in \mathbb{N}} a_{n} e_{n}$. Sejam $P$ a projeção de $C(K)$ sobre $T\left[c_{0}\right]$ como na prova da Proposição 1.7 e $R: c_{0} \rightarrow c_{0}$ o operador shift a direita, definido como $R(x)(0)=0$ e $R(x)(n)=x(n-1)$, se $n>0$. Então, para $g \in C(K)$ e todo $n \in \mathbb{N}$, temos:

$$
\left|T R T^{-1} P\left(e_{n}\right)\left(x_{n+1}\right)-g\left(x_{n+1}\right) e_{n}\left(x_{n+1}\right)\right|=\left|\sum_{n \in \mathbb{N}}\left(e_{n}\left(x_{j}\right) e_{j+1}\left(x_{n+1}\right)\right)\right|=\left|e_{n}\left(x_{n}\right) e_{n+1}\left(x_{n+1}\right)\right|=1 .
$$

Logo, $\left\|\left(T S T^{-1} P\right)\left(e_{n}\right)-g I\left(e_{n}\right)\right\| \geqslant 1$, para todo $n \in \mathbb{N}$, onde $I$ é o operador identidade em $C(K)$. Assim, pelo Teorema 1.15, $T S T^{-1} P-g I$ não é fracamente compacto . Como $g \in C(K)$ é arbitrária, então $T S T^{-1} P$ não é da forma $g I+S$. 


\subsection{Medidas de Radon e Representação de Riesz}

Se $\pi: Y \longrightarrow X$ é uma função contínua entre dois espaços topológicos e $\mu$ é uma medida em $Y$, definimos $\mu \circ \pi^{-1}$ a função definida nos borelianos de $X$ em $\mathbb{R}$ dada por $\mu \circ \pi^{-1}[B]=\mu\left(\pi^{-1}[B]\right)$, para todo $B$ boreliano em $X$.

Denotamos por $M(K)$ o espaço vetorial das medidas de Radon sobre um espaço topológico $K$ e por $\operatorname{Bor}(K)$ a $\sigma$-álgebra de Borel de $K$.

Lema 1.17. Sejam $L$ e $K$ compactos e $\pi: L \rightarrow K$ uma função contínua e sobrejetora. Se $\mu \in$ $M(L)$, então $\mu \circ \pi^{-1} \in M(K)$.

Demonstração: Como $\pi$ é contínua, então para qualquer $B \in \operatorname{Bor}(K)$ o conjunto $\pi^{-1}[B]$ pertence a $\operatorname{Bor}(L)$, garantindo que $\mu \circ \pi^{-1}$ está bem definida. Do fato de $\mu$ ser medida finita segue $\mu \circ \pi^{-1}[K]=\mu[L]<\infty, \mu \circ \pi^{-1}[\emptyset]=0$, e que, para qualquer coleção $\left\{B_{i}: i \in \mathbb{N}\right\}$ de conjuntos disjuntos em $\operatorname{Bor}(X)$, temos $\mu \circ \pi^{-1}\left[\bigcup_{i \in \mathbb{N}} B_{i}\right]=\mu\left[\bigcup_{i \in \mathbb{N}} \pi^{-1}\left[B_{i}\right]\right]=\sum_{i \in \mathbb{N}}\left(\mu \circ \pi^{-1}\right)\left[B_{i}\right]$. Logo, $\mu \circ \pi^{-1}$ é medida finita.

Para provar as condições de regularidade, assumimos primeiro que $\mu \geq 0$. Vejamos que $\mu \circ \pi^{-1}$ satisfaz a propriedade de regularidade interna. Se $V$ é aberto em $K$, pela monotonicidade da medida, $\left(\mu \circ \pi^{-1}\right)(F) \leq\left(\mu \circ \pi^{-1}\right)(V)$, para todo $F \subset V$. Agora dado $\varepsilon>0$, pela regularidade de $\mu$ existe um compacto $A \subset \pi^{-1}[V]$ tal que $\mu(A)>\mu\left(\pi^{-1}[V]\right)-\varepsilon$. Pela continuidade de $\pi, \pi(A)$ é compacto e, como $A \subset \pi^{-1}[\pi[A]] \subset \pi^{-1}[V]$, então $\mu\left(\pi^{-1}(\pi[A])\right)>\mu\left(\pi^{-1}[V]\right)-\varepsilon$, provando a regularidade interior de $\mu \circ \pi^{-1}$.

Para provar que $\mu \circ \pi^{-1}$ satisfaz a regularidade exterior, consideremos $E \in \operatorname{Bor}(K)$ e $\varepsilon>0$. Pela regularidade exterior de $\mu$ existe um aberto $U$ em $L$ contendo $\pi^{-1}[E]$ tal que $\mu\left(\pi^{-1}[E]\right)>\mu(U)-\varepsilon$. Como $\pi^{-1}[E] \cap(L \backslash U)=\emptyset$, então $E \cap \pi(L \backslash U)=\emptyset$. Ou seja, $E \subset K \backslash(\pi(L \backslash E))$, portanto $\pi^{-1}[E] \subset$ $\pi^{-1}[K \backslash \pi(L \backslash U)] \subset U$. Pela monotonicidade de $\mu, \mu\left(\pi^{-1}[E]\right) \leq \mu\left(\pi^{-1}[K \backslash \pi(L \backslash U)]\right) \leq \mu(U)$. Consequentemente, $\left(\mu \circ \pi^{-1}\right)(E)>\left(\mu \circ \pi^{-1}\right)(K \backslash \pi(L \backslash U))-\varepsilon$. Note que $\pi(L \backslash U)$ é fechado, porque $K$ é compacto e Hausdorff e $\pi$ é contínua. Logo $K \backslash \pi(L \backslash U)$ é um aberto em $L$ contendo $E$, provando a regularidade exterior de $\mu \circ \pi^{-1}$.

Finalmente, se $\mu$ é uma medida signada de Radon, então $\mu=\mu^{+}-\mu^{-}$, onde $\mu^{+}$e $\mu^{-}$são medidas de Radon não negativas e, pela primeira parte da prova, $\mu^{+} \circ \pi^{-1}$ e $\mu^{-} \circ \pi^{-1}$ são medidas de Radon, provando que $\mu \circ \pi^{-1}$ é uma medida de Radon.

Lema 1.18. Sejam $\beta<\alpha, L \subset[0,1]^{\alpha}$ compacto e $K=\pi_{\beta, \alpha}[L]$. Se $f \in C(K)$ e $\mu \in M(L)$, então

$$
\int_{K} f d \mu \circ \pi_{\beta, \alpha}^{-1}=\int_{L} f \circ \pi_{\beta, \alpha} d \mu \text {. }
$$

Demonstração: As duas integrais existem porque as funções $f$ e $f \circ \pi_{\beta, \alpha}$ são contínuas e, portanto, limitadas em $K$ e $L$, respetivamente, e as medidas são finitas. Como $f$ é contínua (e, portanto, boreliana) e limitada, existe uma sequência crescente $\left(\phi_{n}\right)_{n \in \mathbb{N}}$ de funções simples definidas em $K$ tais que $\phi_{n} \longrightarrow f$ uniformemente em $K$. Seja $\phi_{n}=\sum_{j=1}^{r_{n}} c_{j}^{(n)} \chi_{E_{j}^{n}}$, onde $E_{j}^{n}$ é boreliano em $K$ e $c_{j}^{(n)}$ é um real positivo. Como $\pi_{\beta, \alpha}$ é contínua, então $\pi_{\beta, \alpha}^{-1}\left(E_{j}^{n}\right)$ é boreliano em $K_{\alpha}$ e, portanto, a 
função $\tilde{\phi}_{n}: L \rightarrow[0,1]$ definida por $\tilde{\phi}_{n}:=\sum_{j=1}^{r_{n}} c_{j}^{(n)} \chi_{\pi_{\beta, \alpha}^{-1}\left[E_{j}^{n}\right]}$ é simples. Note que $\phi_{n} \leq \phi_{n+1}$ implica $\tilde{\phi}_{n} \leq \tilde{\phi}_{n+1}$ e, além disso,

$$
\int \tilde{\phi}_{n} d \mu=\sum_{j=1}^{r_{n}} c_{j}^{(n)} \mu\left(\pi_{\beta, \alpha}^{-1}\left[E_{j}^{n}\right]\right)=\int \phi_{n} d \mu \circ \pi_{\beta, \alpha}^{-1} .
$$

Agora, sejam $\varepsilon>0$ e $N$ inteiro positivo tais que $n>N$ implica $\left\|f-\phi_{n}\right\|<\varepsilon$. Para $n>N$ acontece

$$
\begin{aligned}
\left\|f \circ \pi_{\beta, \alpha}-\tilde{\phi}_{n}\right\| & =\sup \left\{\sup _{y \in \pi_{\beta, \alpha}^{-1}\left[E_{j}^{n}\right]}\left|f \circ \pi_{\beta, \alpha}(y)-\tilde{\phi}_{n}(y)\right|: 1 \leq j \leq r_{n}\right\} \\
& =\sup \left\{\sup _{x \in E_{j}^{n}}\left|f(x)-\phi_{n}(x)\right|: 1 \leq j \leq r_{n}\right\} \\
& =\left\|f-\phi_{n}\right\|<\varepsilon
\end{aligned}
$$

isto é, $\tilde{\phi}_{n} \longrightarrow f \circ \pi_{\beta, \alpha}$ uniformemente. Pelo Teorema da convergência dominada

$$
\int f \circ \pi_{\beta, \alpha} d \mu=\lim _{n \rightarrow \infty} \int \tilde{\phi}_{n} d \mu=\lim _{n \rightarrow \infty} \int \phi_{n} d \mu \circ \pi_{\beta, \alpha}^{-1}=\int f d \mu \circ \pi_{\beta, \alpha}^{-1}
$$

Proposição 1.19 ([Faj07], Lema 1.1). Sejam $K$ um compacto e $\mathcal{B}$ uma base de abertos de $K$ fechada por intersecções finitas. Se $\mu$ e $\nu$ são medidas de Radon sobre $K$ tais que $\left.\mu\right|_{\mathcal{B}}=\left.\nu\right|_{\mathcal{B}}$, então $\mu=\nu$.

Denotaremos por $\mathfrak{B}$ a base de abertos para a topologia de $[0,1]^{\omega_{1}}$ definida por:

$$
\left\{\prod_{\xi<\omega_{1}} V_{\xi}:\left|\left\{n \in \mathbb{N}: V_{\xi} \neq[0,1]\right\}\right|<\omega\right\}
$$

onde cada $V_{\xi}$ é um intervalo aberto em $[0,1]$ com extremos racionais. Adicionalmente, denotamos por $\mathfrak{B}_{\alpha}$ o subconjunto $\left\{\prod_{\xi<\omega_{1}} V_{\xi} \in \mathfrak{B}: \forall \xi \geq \alpha\left(V_{\xi}=[0,1]\right)\right\}$ de $\mathfrak{B}$ e para $\alpha \leq \omega_{1}$, por $\mathcal{B}_{\alpha}$ a base da topologia de $[0,1]^{\alpha}$ formada por $\left\{\prod_{\xi<\alpha} V_{\xi}:\left|\left\{n \in \mathbb{N}: V_{\xi} \neq[0,1]\right\}\right|<\omega\right\}$.

Note que a função $\pi_{\alpha}$ restrita a $\mathfrak{B}_{\alpha}$ é uma bijeção em $\mathcal{B}_{\alpha}$. Pelo Lema 1.17 e a Proposição 1.19, se $\mu$ é uma medida de Radon podemos identificar a função $\left.\mu\right|_{\mathfrak{B}_{\alpha}}$ com a medida $\mu \circ \pi_{\alpha}^{-1}$ em $[0,1]^{\alpha}$. Por abuso de notação escreveremos às vezes $\left.\mu\right|_{\mathcal{B}_{\alpha}}$ para nos referir a medida $\left.\mu \circ \pi_{\alpha}^{-1}\right|_{\mathcal{B}_{\alpha}}$.

O próximo lema garante que todo subconjunto boreliano do limite de um sistema inverso de espaços topológicos Hausdorff é um boreliano do espaço produto dos espaços topológicos.

Lema 1.20. Sejam $X$ um espaço topológico. Se $E \in \operatorname{Bor}(X)$, então Bor $(E)=\{E \cap B: B \in$ Bor $(X)\}$, considerando E com a topologia do subespaço.

Demonstração: $\{E \cap B: B \in B \operatorname{Bor}(X)\}$ contém todos os fechados (abertos ) de $E$. Logo, $\operatorname{Bor}(E) \subset\{E \cap B: B \in \operatorname{Bor}(X)\}$.

Para demonstrar a recíproca, considere $i: E \rightarrow X$ a inclusão. Pela continuidade de $i$, para todo $B \in \operatorname{Bor}(X)$ temos $i^{-1}[B] \in \operatorname{Bor}(E)$ e, portanto, $E \cap B=i^{-1}[B] \in \operatorname{Bor}(E)$. Logo, $\{E \cap B: B \in$ 
$\operatorname{Bor}(X)\} \subset \operatorname{Bor}(E)$.

Se $E \subset X$ e $E \in \operatorname{Bor}(X)$, então, para todo $B \in \operatorname{Bor}(X)$, temos $B \cap E \in \operatorname{Bor}(X)$ e, portanto, $\{B \cap E: B \in \operatorname{Bor}(X)\}=\{B \in \operatorname{Bor}(X): B \subset E\}$

Observação 2. Seja $K_{\omega_{1}} \subset[0,1]^{\omega_{1}}$ compacto. O lema anterior implica que Bor $\left(K_{\omega_{1}}\right) \subset \operatorname{Bor}\left([0,1]^{\omega_{1}}\right)$. Além disso, se $\mu: \operatorname{Bor}\left([0,1]^{\omega_{1}}\right) \rightarrow \mathbb{R}$ é uma medida, então $\left.\mu\right|_{K_{\omega_{1}}}:$ Bor $\left(K_{\omega_{1}}\right) \rightarrow \mathbb{R}$ definida por $\left.\mu\right|_{K_{\omega_{1}}}[B]=\mu[B]$ é uma medida sobre $K_{\omega_{1}}$

Parte de nosso trabalho do Capítulo 5, será feito usando medidas de Radon, o que é possível em parte devido ao Teorema de Gantmacher, que afirma que um operador linear $T$ entre espaços de Banach é fracamente compacto se, e somente, seu adjunto $T^{*}$ também é fracamente compacto.

Uma vantagem de trabalhar como o espaço dual de $C(K)$ é que cada funcional linear de $C(K)$ pode ser visto como uma medida de Radon.

Teorema 1.21 (Teorema de Representação de Riesz). Se $K$ é um espaço compacto, então para cada funcional linear contínuo $\phi$ sobre $C(K)$ existe uma medida de Radon $\mu$ em $K$ tal que

$$
\phi(f)=\int f(x) d \mu(x)
$$

para todo $f \in C(K)$. A norma do funcional $\phi$ é igual a variação total da medida $\mu$ em $K$ i.e

$$
\|\phi\|=|\mu|(K)
$$

Mais ainda, a medida $\mu$ é não negativa se, e somente se, o funcional $\phi$ é não negativo. Ou seja $\mu \geq 0$ se, e somente se, $f \geq 0$ implica $\phi(f) \geq 0$.

\subsection{Princípio}

Nesta seção abordaremos Princípio $\diamond$, um axioma da teoria dos conjuntos que afirma a existência de uma sequência de objetos de tamanho crescente, tal que qualquer objeto grande no final é frequentemente antecipado pelos objetos da sequência.

$\mathrm{O}$ Princípio $\diamond$ é relativamente consistente com $Z F C$, é válido no universo construtível e implica a Hipótese do Continuum (CH). Para os detalhes indicamos [Jec03] ou [Kun80].

Antes de estabelecer o princípio, lembramos que um subconjunto $C$ de $\omega_{1}$ é fechado e ilimitado, se é não enumerável e o supremo em $\omega_{1}$ de qualquer sequência enumerável crescente em $C$ pertence a $C$. Um subconjunto $S$ de $\omega_{1}$ é estacionário se intersecta todos os conjuntos fechados e ilimitados em $\omega_{1}$.

Princípio $\diamond$. Existe uma sequência $\left(X_{\alpha}\right)_{\alpha \in \omega_{1}}$, chamada de $\diamond$-sequência, tal que $X_{\alpha} \subset \alpha$ para todo $\alpha \in \omega_{1}$, e

$$
\left\{\alpha \in \omega_{1}: X \cap \alpha=X_{\alpha}\right\}
$$

é estacionário, para todo $X \subset \omega_{1}$.

O próximo lema, retirado de [Faj12], será usado no Capítulo 5.

Lema 1.22 (Lema 4.2, [Faj12] ). O Princípio $\diamond$ implica: 
(i) Se $\left(B_{\alpha}\right)_{\alpha<\omega_{1}}$ é uma sequência de cardinalidade $\omega_{1}$, existe uma sequência $\left(x_{\alpha}\right)_{\alpha<\omega_{1}}$ tal que $x_{\alpha} \in \prod_{\beta<\alpha} B_{\beta}$ para todo $\alpha<\omega_{1}$, e $\left\{\alpha<\omega_{1}:\left.x\right|_{\alpha}=x_{\alpha}\right\}$ é estacionário para todo $x \in$ $\prod_{\alpha<\omega_{1}} B_{\alpha}$.

(ii) Existe uma sequência $\left\{x_{n}(\alpha): n \in \mathbb{N}, \alpha<\omega_{1}\right\}$ e tal que $x_{n}(\alpha) \in[0,1]^{\alpha}$ para todo $\alpha<\omega_{1}$, e $\left\{\alpha \in \omega_{1}: \forall n \in \mathbb{N}\left(\left.x_{n}\right|_{\alpha}=x_{n}(\alpha)\right)\right\}$ é estacionário para todas as sequências $\left(x_{n}\right)_{n \in \mathbb{N}}$ em $[0,1]^{\omega_{1}}$.

(iii) Existe $\left(x_{\alpha}\right)_{\alpha<\omega_{1}}$ tal que $x_{\alpha} \in[0,1]^{\alpha \times \alpha}$ para todo $\alpha<\omega_{1}$, e $\left\{\alpha<\omega_{1}:\left.x\right|_{\alpha \times \alpha}=x_{\alpha}\right\}$ é estacionário para todo $x \in[0,1]^{\omega_{1} \times \omega_{1}}$.

(iv) Existe uma sequência $\left(A_{\alpha}\right)_{\alpha<\omega_{1}}$ de subconjuntos de $\omega_{1}$ tal que $\left\{\alpha \in \omega_{1}:\left\{\left.z_{\beta}\right|_{\alpha}: \beta<\alpha\right\}=A_{\alpha}\right\}$ sempre que $\left(z_{\beta}\right)_{\beta \in \omega_{1}}$ é uma sequência em $[0,1]^{\omega_{1}}$.

Lema $1.23(Z F C+\diamond)$. Existe $\left(\mu_{\alpha}\right)_{\alpha<\omega_{1}}$ tal que $\mu_{\alpha} \in \mathbb{R}^{\mathfrak{B}_{\alpha}}$ e para toda $\mu \in \mathbb{R}^{\mathfrak{B}_{\omega_{1}}}$ o conjunto $\left\{\alpha<\omega_{1}:\left.\mu\right|_{\mathfrak{B}_{\alpha}}=\mu_{\alpha}\right\}$ é estacionário.

Demonstração: Para cada $\alpha \leq \omega_{1}$, denotamos por $M_{\alpha}$ o conjunto das funções $\mathbb{R}^{\mathfrak{B}_{\alpha}}$.

Usando o Lema 1.22 para $M_{\alpha}$ no lugar de $B_{\alpha}$, tome $\left(f_{\alpha}\right)_{\alpha<\omega_{1}}, \diamond$-sequência tal que $f_{\alpha} \in \prod_{\beta<\alpha} M_{\beta}$ e para todo $f \in \prod_{\alpha<\omega_{1}} M_{\alpha}$, o conjunto $\left\{\alpha<\omega_{1}:\left.f\right|_{\alpha}=f_{\alpha}\right\}$ é estacionário.

Defina $\mu_{\alpha} \in M_{\alpha}$ como $\mu_{\alpha}(V)=f_{\alpha}(\beta)(V)$, onde $\beta=\min \left\{\gamma<\alpha: V \in \mathfrak{B}_{\gamma}\right\}$ para $\alpha \in \operatorname{Lim}\left(\omega_{1}\right)$, e para $\alpha$ sucessor tome $\mu_{\alpha}$ qualquer.

Seja $\mu \in M_{\omega_{1}}$, tome $f \in \prod_{\alpha<\omega_{1}} M_{\alpha}$ dada por $f(\alpha)=\left.\mu\right|_{\mathfrak{B}_{\alpha}}$. Vamos mostrar que se $\left.f\right|_{\alpha}=f_{\alpha}$ e $\alpha \in \operatorname{Lim}\left(\omega_{1}\right)$, então $\left.\mu\right|_{\mathfrak{B}_{\alpha}}=\mu_{\alpha}$.

Suponha $\alpha \in \operatorname{Lim}\left(\omega_{1}\right)$ e $\left.f\right|_{\alpha}=f_{\alpha}$. Seja $V \in \mathfrak{B}_{\alpha}$ e tome $\beta=\min \left\{\gamma<\alpha: V \in \mathfrak{B}_{\gamma}\right\}$, temos $\beta<\alpha$ e $\mu_{\alpha}(V)=f_{\alpha}(\beta)(V)=f(\beta)(V)=\mu(V)$ pois $V \in \mathfrak{B}_{\beta}$. Como $\operatorname{Lim}\left(\omega_{1}\right)$ é fechado e ilimitado, $\left\{\alpha \in \operatorname{Lim}\left(\omega_{1}\right):\left.f\right|_{\alpha}=f_{\alpha}\right\}$ é estacionário e contido em $\left\{\alpha<\omega_{1}:\left.\mu\right|_{\mathfrak{B}_{\alpha}}=\mu_{\alpha}\right\}$, provando que esse último também é estacionário. 


\section{Capítulo 2}

\section{Espaços de Koszmider e fracamente Koszmider}

A definição de espaços $C(K)$ com poucos operadores foi apresentada por Koszmider, em [Kos04]. Neste capítulo são estudadas algumas propriedades do espaço compacto $K$ quando $C(K)$ tem poucos operadores. O capítulo está dividido em três seções, a primeira dedicada as definições e propriedades dos espaços Koszmider e fracamente Koszmider. Nessa seção apenas são originais os Lemas 2.10, 2.11, e 2.12, sendo este último apenas uma leve mudança da caraterização apresentada por Koszmider em [Kos04]. Nas Seções 2 e 3 são apresentadas condições necessárias e suficientes para $C(K)$ ter poucos operadores, respectivamente. As Proposições 2.14 e 2.15 são originais. Os Teoremas 2.18 e 2.19 são simples adaptações dos trabalhos de [Kos04] e [Faj12].

\subsection{Operadores multiplicadores fracos e multiplicações fracas}

Diremos que um espaço de Banach real $X$ tem poucos operadores no sentido de Argyros e Haydon se para todo operador $T \in \mathcal{L}(X)$ existem $\lambda \in \mathbb{R}$ e $C$ um operador compacto sobre $X$ tais que

$$
T=\lambda I+C
$$

Adicionalmente, diremos que um espaço de Banach $X$ tem poucos operadores no sentido de Gowers e Maurey se para todo operador $T$ em $\mathcal{L}(X)$ existem $\lambda \in \mathbb{R}$ e um operador estritamente singular $S$ sobre $X$ tais que

$$
T=\lambda I+S
$$

Koszmider em [Kos04] provou que não existem espaços $C(K)$ com poucos operadores no sentido de Gowers e Maurey. Nós apresentamos os detalhes deste fato.

Proposição 2.1. Para todo espaço compacto infinito $K$ existe um operador $T \in \mathcal{L}(C(K))$ que não é da forma $\lambda I+S$, onde $\lambda \in \mathbb{R}, I$ é o operador identidade e $S$ é um operador estritamente singular.

Demonstração: Seja $K$ um espaço compacto. Se $K$ é disperso, o resultado segue do Corolário 1.16, porque $\lambda I$ é operador multiplicação pela função constante $\lambda$.

Se $K$ não é disperso, então contém um subconjunto $E$ sem pontos isolados. Tome $x, y$ pontos diferentes em $E$ e sejam $V$ e $W$ abertos disjuntos contendo $x$ e $y$ respectivamente. Então $K \backslash V$ 
é infinito, pois $E$ não tem pontos isolados. Pela normalidade de $K$, existe um aberto $U$ tal que $x \in U \subset \bar{U} \subset V$. O aberto $U$ é infinito porque $x$ não é isolado. Assim, pelo Lema de Urysohn existe uma função contínua $f$ de $K$ em $\left.[0,1] \operatorname{com} f\right|_{\bar{U}}=0$ e $\left.f\right|_{K \backslash V}=1$.

Denotemos por $\mathcal{N}$ o subespaço de $C(K)$ formado pelas funções que se anulam em $K \backslash U$. Note que, se $\lambda g-f g=0$ e $g \in \mathcal{N}$, então $\lambda=0$ ou $g=0$. Portanto, para $\lambda \neq 0$ o operador $\lambda I-f I$ : $C(K) \rightarrow C(K)$ restrito a $\mathcal{N}$ é injetor, logo não é estritamente singular e, consequentemente, o operador $f I: C(K) \rightarrow C(K)$ é um exemplo de um operador que não é da forma $\lambda I+S$, onde $S$ é estritamente singular.

As definições de operador multiplicador fraco e multiplicação fraca são devidas a Koszmider. Apareceram primeiro em [Kos04] motivados pela impossibilidade de que existam espaços de Banach $C(K)$ com poucos operadores no sentido de Gowers e Maurey ou de Argyros e Haydon.

Lembramos que duas funções $f$ e $g$ de um espaço topológico $X$ em $\mathbb{R}$ são disjuntas se $f \cdot g=0$. Note que essa condição é mais fraca do que ter suportes disjuntos, que, por sua vez é mais fraca do que os suportes estarem contidos em abertos disjuntos (i.e., existem abertos disjuntos $U$ e $V$ em $X$ tais que $\operatorname{supp}(f) \subset U$ e $\operatorname{supp}(g) \subset V)$.

Definição 2.2. Seja $K$ um espaço compacto. Dizemos que um operador $T: C(K) \rightarrow C(K)$ é um multiplicador fraco se para toda sequência $\left(f_{n}\right)_{n \in \mathbb{N}}$ de funções em $C(K)$ duas a duas disjuntas $\left(f_{n} \cdot f_{m}=0\right.$, sempre que $\left.m \neq n\right)$ e toda sequência $\left(x_{n}\right)_{n \in \mathbb{N}}$ em $K$ tal que $f_{n}\left(x_{n}\right)=0$, temos

$$
\lim _{n \rightarrow \infty} T\left(f_{n}\right)\left(x_{n}\right)=0
$$

Exemplo 1. Todo operador em $C(K)$ fracamente compacto é multiplicador fraco. Em particular, os operadores compactos também são multiplicadores fracos.

A prova do exemplo é consequência do Teorema 1.15, que garante que, se $T$ é fracamente compacto, então $\lim _{n \rightarrow \infty} T\left(f_{n}\right)=0$ para qualquer sequência limitada $\left(f_{n}\right)_{n \in \mathbb{N}}$ de funções de $K$ em $\mathbb{R}$, duas a duas disjuntas, o que implica que $\lim _{n \rightarrow \infty} T\left(f_{n}\right)\left(x_{n}\right)=0$.

Definição 2.3. Seja $K$ um espaço compacto. Um operador $T: C(K) \rightarrow C(K)$ é dito uma multiplicação fraca se existem $g \in C(K)$ e um operador estritamente singular $S$ tais que $T=g I+S$, onde $I$ é o operador identidade.

Mostraremos que a classe dos operadores que são multiplicações fracas em um espaço $C(K)$ está contida na classe dos operadores que são multiplicadores fracos, e essa inclusão pode ser própria.

Proposição 2.4. Se $T: C(K) \rightarrow C(K)$ é uma multiplicação fraca, então $T$ é multiplicador fraco.

Demonstração: Seja $T: C(K) \rightarrow C(K)$ o operador $g I+S$, para $g \in C(K)$ e $S$ fracamente compacto. Sejam $\left(f_{n}\right)_{n \in \mathbb{N}}$ uma sequência limitada de funções em $C(K)$, duas a duas disjuntas, e $\left(x_{n}\right)_{n \in \mathbb{N}}$ uma sequência em $K$ tais que $f_{n}\left(x_{n}\right)=0$, para todo $n \in \mathbb{N}$. Temos que $T\left(f_{n}\right)\left(x_{n}\right)=$ $g f_{n}\left(x_{n}\right)+S\left(f_{n}\right)\left(x_{n}\right)=S\left(f_{n}\right)\left(x_{n}\right)$. Pelo Teorema 1.15, $\left\|S\left(f_{n}\right)\right\|$ converge a 0 e, portanto, $S\left(f_{n}\right)\left(x_{n}\right)$ também converge a 0 , provando que $T$ é multiplicador fraco.

O próximo exemplo é tomado de [Kos10], e mostra que a inclusão acima pode ser própria.

Exemplo 2. $O$ operador $T: C[0,1] \rightarrow C[0,1]$ definido por

$$
T(f)=\chi_{[0,1 / 2]}(f-f(1 / 2))
$$


é multiplicador fraco mas não multiplicação fraca.

De fato, o operador $T$ é multiplicador fraco porque, para qualquer sequência limitada de funções contínuas $f_{n}:[0,1] \rightarrow \mathbb{R}$, duas a duas disjuntas, existe $n_{0}$ tal que $f_{n}(1 / 2)=0$, para todo $n>n_{0}$, e, assim, $T\left(f_{n}\right)\left(x_{n}\right)=0$, para todo $n>n_{0}$ e qualquer sequência $\left(x_{n}\right)_{n \in \mathbb{N}}$ em $[0,1]$ que verifique $f_{n}\left(x_{n}\right)=0$.

Para verificar que $T$ não é multiplicação fraca, suponhamos que exista $g \in C([0,1])$ tal que $T-g I$ é um operador fracamente compacto em $C([0,1])$. Usando o Lema de Urysohn podemos achar sequências limitadas $\left(f_{n}\right)_{n \in \mathbb{N}}$ e $\left(f_{n}^{\prime}\right)_{n \in \mathbb{N}}$ de funções duas a duas disjuntas, tais que $f_{n}\left(\frac{n-2}{2 n}\right)=1=f_{n}^{\prime}\left(\frac{n+2}{2 n}\right)$ e $f_{n}([1 / 2,1])=\{0\}=f_{n}^{\prime}([0,1 / 2])$, para todo $n \in \mathbb{N}$. Pelo Teorema 1.15, temos $\lim _{n \rightarrow \infty}\left\|\chi_{[0,1 / 2]} f_{n}-g f_{n}\right\|=0$ e, portanto, $\lim _{n \rightarrow \infty}\left|1-g\left(\frac{n-2}{2 n}\right)\right|=0$. Logo, da continuidade de $g$ segue que $g(1 / 2)=1$. Analogamente, pelo Teorema 1.15, $\lim _{n \rightarrow \infty}\left\|-g f_{n}^{\prime}\right\|=0 \operatorname{logo} \lim _{n \rightarrow \infty}\left|g\left(\frac{n+2}{2 n}\right)\right|=0$ e, novamente pela continuidade da $g, g(1 / 2)=0$, chegando numa contradição e completando a prova de que $T$ não é multiplicação fraca.

Em [Kos04] é usada uma caracterização de multiplicadores fracos que deixa mais evidente a semelhança dessa definição com a de multiplicações fracas. Antes de enunciarmos essa caracterização, precisamos estabelecer algumas notações.

Se $g$ é uma função boreliana limitada de $K$ em $\mathbb{R}$, seguindo a notação usada em [Kos04] definimos o operador $g I$ em $M(K)$ (o espaço das medidas de Radon sobre $K$ ) que associa a cada $\mu \in M(K)$ a medida $\nu \in M(K)$ dada por $\nu(B)=\int g \cdot \chi_{B} d \mu$, para todo boreliano $B \subset K$.

Pelo Teorema de representação de Riesz, identificando os funcionais lineares em $C(K)^{*}$ com as medidas em $M(K)$, se $g \in C(K)$ temos $(g I)^{*}=g I$. Ou seja, para toda $f \in C(K)$,

$$
\int f d(g I)^{*}(\mu)=\int g f d \mu
$$

Assim $(g I)^{*}$ envia o funcional que é a integração em relação a medida $\mu$ ao funcional que é a integração da multiplicação por $g$ em relação a medida $\mu$.

Koszmider mostra que a definição de multiplicador fraco se assemelha à definição de multiplicação fraca no dual de $C(K)$.

Teorema 2.5 (Teorema 2.2, [Kos04]). Seja $K$ um espaço compacto. Um operador $T: C(K) \rightarrow$ $C(K)$ é multiplicador fraco se, e somente se, existem uma função boreliana $g: K \rightarrow \mathbb{R}$ limitada $e$ um operador $S: C(K)^{*} \rightarrow C(K)^{*}$ fracamente compacto tais que $T^{*}=g I+S$.

Com essa caracterização, a Proposição 2.4 segue direto do Teorema de Gantmacher, que afirma que um operador linear $S$ em um espaço de Banach $X$ é fracamente compacto se, e somente, o operador adjunto $S^{*}$ é fracamente compacto em $X^{*}$.

Os espaços topológicos $K$ tais que todo operador linear sobre $C(K)$ são multiplicadores fracos ou multiplicações fracas foram chamados por Koszmider em [Kos04] de espaços com poucos operadores. A seguinte definição foi introduzida por Plebaneck em [Ple04] e por Schlackow em [Sch08a]

Definição 2.6. Um espaço topológico compacto $K$ é dito:

(i) espaço de Koszmider se todo operador $T$ sobre $C(K)$ é multiplicação fraca. 
(ii) espaço fracamente Koszmider se, e somente se, todo operador sobre $C(K)$ é multiplicador fraco.

Segue imediatamente da Proposição 2.4 que todo espaço de Koszmider é fracamente Koszmider. A condição em que o as duas definições são equivalentes aparecem no próximo lema. Lembramos que um subespaço $Y$ de um espaço topológico $X$ é $C^{*}$-imerso em $X$ se toda função contínua limitada em $Y$ pode ser estendida a uma função continua limitada em $X$.

Lema 2.7 (Teorema 2.7, [Kos04]). Seja K um espaço compacto. Então K é um espaço de Koszmider se, e somente se, $K$ é fracamente Koszmider e, para todo $x \in K$, o espaço $K \backslash\{x\}$ é $C^{*}$-imerso em $K$.

Para frente usaremos uma propriedade topológica considerada em [Kos04] e usada para construir um espaço de Koszmider. Ela será fundamental em algumas partes do Capítulo 6, e por isso a destacamos na seguinte definição.

Definição 2.8. Um espaço compacto $K$ contém abertos-borboleta se existem abertos disjuntos $U$ e $V$ em $K$ tais que $\bar{U} \cap \bar{V}=\{x\}$ para algum $x \in K$.

Os espaços de Koszmider construídos em [Kos04] não contém abertos-borboleta.

Lema 2.9 (Lema 2.8, [Kos04]). Se $K$ não contém abertos-borboleta, então para todo $x \in K$ o espaço $K \backslash\{x\}$ é $C^{*}$-imerso em $K$.

Em geral os subespaços de um espaço que não contém abertos-borboleta podem conter abertosborboleta. Por exemplo, considere $\mathbb{R} \times R$ onde $R$ é o conjunto dos reais munido da topologia trivial. Temos que $\mathbb{R} \times R$ não contém aberto-borboleta pois os abertos contendo um ponto $\left(b_{1}, b_{2}\right)$ são os mesmos que contém $\left\{b_{1}\right\} \times R$. Mesmo assim, o subespaço $\mathbb{R} \times\{0\}$ contém muitos abertos-borboleta.

Por outro lado, os subespaços que são fechos de abertos herdam a propriedade de não conter abertos-borboleta.

Lema 2.10. Seja $X$ um espaço topológico e $W$ um aberto em $X$. Se $X$ não possui abertos-borboleta então $\bar{W}$ não possui abertos-borboleta.

Demonstração: Sejam $U_{1}, U_{2} \subset \bar{W}$ abertos (na topologia do subespaço) disjuntos tais que $\overline{U_{1}} \cap$ $\overline{U_{2}}=\left\{x_{0}\right\}$. Para cada $i \in\{1,2\}$, sejam $V_{i}$ aberto em $X$ tal que $V_{i} \cap \bar{W}=U_{i}$ e $W_{i}=V_{i} \cap W$. Assim, $W_{i}$ é um aberto em $X$ contido em $U_{i}$ e é não vazio, porque $V_{i} \cap \bar{W} \neq \emptyset$.

Agora vejamos que $\overline{W_{1}} \cap \overline{W_{2}}=\left\{x_{0}\right\}$. Note que $\overline{W_{i}} \subset \overline{U_{i}}$. Logo, $\overline{W_{1}} \cap \overline{W_{2}} \subset \overline{U_{1}} \cap \overline{U_{2}}=\left\{x_{0}\right\}$. Por outro lado, como $x_{0} \in \overline{U_{i}}$, se $V$ é um aberto contendo $x_{0}$, então $V \cap V_{i} \cap \bar{W}=V \cap U_{i} \neq \emptyset$. Logo, existe $x \in\left(V \cap V_{i}\right) \cap W$ e, portanto, $V \cap\left(V_{i} \cap W\right)=V \cap W_{i} \neq \emptyset$. Logo, $x_{0} \in \overline{W_{i}}$, isto é, $x_{0} \in \overline{W_{1}} \cap \overline{W_{2}}$

A ausência de abertos-borboleta num espaço normal implica que os subconjuntos abertos conexos, permanecem conexos quando retirado um ponto.

Lema 2.11. Sejam $K$ compacto Hausdorff sem abertos-borboleta e $V \subset K$ um aberto. Então, se $V$ é conexo, $V \backslash\{x\}$ é conexo, para todo $x \in V$. 
Demonstração: Sejam $V$ aberto e $x \in V$. Suponha que $V \backslash\{x\}$ é desconexo. Existem $U_{1}$ e $U_{2}$ abertos de $K$ tais que $U_{1} \cap(V \backslash\{x\}) \neq \emptyset, U_{2} \cap(V \backslash\{x\}) \neq \emptyset, V \backslash\{x\} \subset U_{1} \cup U_{2}$, e $U_{1} \cap U_{2} \cap(V \backslash\{x\})=\emptyset$.

Note que se $x \in U_{2}$ então $U_{1} \backslash\{x\}$ é aberto não vazio e assim $U_{1} \backslash\{x\}$ e $U_{2}$ formam uma separação de $V$ contradizendo que é conexo. Analogamente se $x \in U_{1}$, portanto $x \notin U_{1} \cup U_{2}$.

Se $x \notin \overline{U_{1}} \cap \overline{U_{2}}$ então podemos supor $x \notin \overline{U_{1}}$ portanto existe um aberto $O$ em $V$ contendo a $x$ tal que $O \cap U_{1}=\emptyset$ mas esto implica que $U_{1}$ e $U_{2} \cup O$ são uma separação de $V$ contradizendo a conexidade.

Pela normalidade do $K$, existe $W$ aberto em $K$ tal que $x \in W \subset \bar{W} \subset V$. Como $x \in \overline{U_{1}} \cap \overline{U_{2}}$ então $U_{1} \cap W \neq \emptyset$ e $U_{2} \cap W \neq \emptyset$; mais ainda

$$
x \in \overline{W \cap U_{1}} \cap \overline{W \cap U_{2}},
$$

caso contrario, podemos supor $x \notin \overline{W \cap U_{1}}$ logo existe um aberto $Z_{x}$ contendo $x$ tal que $Z_{x} \cap W \cap$ $U_{1}=\emptyset$ o que contradiz $x \in \overline{U_{1}}$ porque $Z_{x} \cap W$ é um aberto (tanto em $K$ quanto em $V$ ) contendo $x$.

Agora seja $y \in V \backslash\{x\}$, então $y \in U_{1}$ ou $y \in U_{2}$. Se $y \in U_{1}$ então $y \notin \overline{W \cap U_{2}}$ porque $U_{1}$ é um aberto em $K$ (note que $V \cap U_{1}$ é aberto em $V$ ) que não interseca $U_{2}$. Analogamente se $y \in U_{2}$ então $y \notin \overline{W \cap U_{1}}$.

Seja $V_{1}=U_{1} \cap W$ e $V_{2}=U_{2} \cap W$. Como $\overline{V_{1}} \cap \overline{V_{2}} \subset \bar{W} \subset V$ então $\overline{V_{1}} \cap \overline{V_{2}}=\{x\}$.

Agora provaremos que na definição de operador multiplicador fraco é possível trocar sequências limitadas de funções em $C(K)$, duas a duas, disjuntas por sequências de funções em $C(K)$, com suportes contidos em abertos disjuntos.

Lema 2.12. Sejam $K$ um espaço compacto e $T: C(K) \rightarrow C(K)$ um operador linear e contínuo. Temos que $T$ é multiplicador fraco se, e somente se, para toda sequência $\left(f_{n}\right)_{n \in \mathbb{N}}$ de funções contínuas de $K$ em $[0,1]$ com suportes contidos em abertos disjuntos e toda sequência $\left(x_{n}\right)_{n \in \mathbb{N}}$ de pontos em $K$ tal que $f_{n}\left(x_{n}\right)=0$ para todo $n \in \mathbb{N}$, acontece $\lim _{n \rightarrow \infty} T\left(f_{n}\right)\left(x_{n}\right)=0$

Demonstração: A implicação direta é imediata da definição de operador multiplicador fraco. Reciprocamente, precisamos provar que podemos supor que as funções têm suportes disjuntos separados por abertos e com imagens contidas em $[0,1]$.

Seja $T: C(K) \longrightarrow C(K)$ não multiplicador fraco. Fixe $\varepsilon>0,\left(g_{n}\right)_{n \in N}$ uma sequência limitada de funções contínuas de $K$ em $\mathbb{R}$, duas a duas disjuntas, e $\left(x_{n}\right)_{n \in \mathbb{N}}$ uma sequência de pontos distintos de $K$ tais que $g_{n}\left(x_{n}\right)=0$, para todo $n \in \mathbb{N}$, e $\left|T\left(g_{n}\right)\left(x_{n}\right)\right|>\varepsilon$, para todo $n \in \mathbb{N}$. Como a sequência é limitada, dividindo pelo supremo de $\left\|g_{n}\right\|$ podemos assumir que $g_{n}$ tem imagem contida em $[-1,1]$. Decompondo $g_{n}=g_{n}^{\prime}-g_{n}^{\prime \prime}$, onde $g_{n}^{\prime}, g_{n}^{\prime \prime}: K \longrightarrow[0,1]$ são disjuntas, podemos tomar uma dessas funções no lugar de $g_{n}$ e trocar $\varepsilon$ por $\frac{\varepsilon}{2}$. Dessa forma, podemos assumir que $g_{n}$ tem imagem contida em $[0,1]$.

Defina $V_{n}=\left\{x \in K: g_{n}(x) \neq 0\right\}$. Temos que $V_{n}$ são dois a dois disjuntos. Pela continuidade de $T$, para cada $n \in \mathbb{N}$ existe $\delta_{n}>0$ tal que, se $\left|f-g_{n}\right|<\delta_{n}$, então $\varepsilon / 2>\mid T(f)-$ $T\left(g_{n}\right)|\geq|\left|T\left(g_{n}\right)\left(x_{n}\right)\right|-\left|T(f)\left(x_{n}\right)\right||>\varepsilon-| T(f)\left(x_{n}\right) \mid$. Logo, novamente trocando $\varepsilon$ por $\varepsilon / 2$, tem-se $\left|T(f)\left(x_{n}\right)\right|>\varepsilon$. Defina $F_{n}=\left\{x \in K:\left|g_{n}(x)\right| \geq \delta_{n}\right\}$. Temos que $F_{n}$ é fechado e, reduzindo $\delta_{n}$, se 
necessário, podemos assumir que $F_{n}$ é não vazio. Claramente, $F_{n} \subset V_{n}$. Usando normalidade, existem abertos $W_{n}$ tais que $F_{n} \subset W_{n} \subset \bar{W}_{n} \subset V_{n}$. Usando o Lema de Urysohn, fixe $h_{n}: K \longrightarrow[0,1]$ tal que $\left.h_{n}\right|_{K \backslash W_{n}}=0$ e $\left.h_{n}\right|_{F_{n}}=1$. Defina $f_{n}=g_{n} \cdot h_{n}$. Temos que $\operatorname{supp}\left(f_{n}\right) \subset \overline{W_{n}} \subset V_{n}$. Logo, os suportes são disjuntos. Temos também que $\left.f_{n}\right|_{F_{n}}=g_{n}$. Verifica-se facilmente que $\left|g_{n}-f_{n}\right|<\delta_{n}$ e, portanto, $\left|T\left(f_{n}\right)\left(x_{n}\right)\right|>\varepsilon$.

Schlakow, em [Sch08b], demostrou, que na definição de operador multiplicador fraco, é possível trocar $x_{n} \in K \backslash\left\{x \in K: f_{n}(x)=0\right\}$ por $x_{n} \in K \backslash \operatorname{supp}\left(f_{n}\right)$. Os operadores multiplicadores fracos são chamados por alguns autores de operadores "Centrípetos", ver por exemplo, [Sch08a] e [Ple04]. Transcrevendo o enunciado do lema anterior para a definição de espaços fracamente Koszmider temos a seguinte equivalência.

Lema 2.13. Seja K um espaço topológico Hausdorff e compacto. São equivalentes:

(a) K é fracamente Koszmider;

(b) Se $\left(f_{n}\right)_{n \in \mathbb{N}}$ é uma sequência de funções contínuas de $K$ em $[0,1]$ para a qual existe uma sequência $\left(V_{n}\right)_{n \in \mathbb{N}}$ de abertos disjuntos satisfazendo $\operatorname{supp}\left(f_{n}\right) \subset V_{n}$, para todo $n \in \mathbb{N}$, e $\left(x_{n}\right)_{n \in N}$ é uma sequência em $K$ tal que $f_{n}\left(x_{n}\right)=0$, para todo $n \in N$, então para todo operador $T: C(K) \longrightarrow C(K)$ temos $T\left(f_{n}\right)\left(x_{n}\right) \rightarrow 0$.

\subsection{Condições necessárias para $C(K)$ ter poucos operadores}

Os espaços compactos $K$ tais que $C(K)$ tem poucos operadores também são interessantes do ponto de vista topológico. Por exemplo, Schlackow, em [Sch08a], demonstrou que os espaços de Koszmider conexos são fortemente rígidos, o que significa que as únicas funções contínuas de $K$ nele mesmo são as funções constantes e a identidade.

Previamente, Koszmider em [Kos04] observou que o seu exemplo de um espaço compacto conexo $K$ com $C(K)$ indecomponível é fortemente rígido. Nesta seção nós estudamos a topologia dos espaços fracamente Koszmider.

Proposição 2.14. Sejam K um espaço fracamente Koszmider sem pontos isolados, $U_{1}$ e $U_{2}$ abertos disjuntos de $K$ e $f$ uma função contínua de domínio $\bar{U}_{1}$ e imagem contida em $U_{2}$. Então, para todo $x \in U_{1}$, existe um aberto $U \subset U_{1}$ tal que $x \in U$ e $f[U]$ é unitário.

Demonstração: Sejam $K, U_{1}, U_{2}$ e $f$ como na hipótese do enunciado e fixe $x \in U_{1}$. Suponha que não exista $U$ como no enunciado.

Tome $W_{2}$ um aberto de $K$ tal que $f(x) \in W_{2} \subset \bar{W}_{2} \subset U_{2}$. Usando continuidade, tome $U \subset U_{1}$ tal que $x \in U$ e $f[U] \subset W_{2}$. Como o espaço é Hausdorff, se $f[U]$ é finito conseguimos refinar $U$ de modo que $f[U]$ seja unitário. Como assumimos que não existe tal $U$, temos que $f[U]$ é infinito.

Seja $\left(y_{n}\right)_{n \in \omega}$ uma sequência relativamente discreta em $f[U]$. Seja $\left(x_{n}\right)_{n \in \omega}$ uma sequência em $U_{1}$ tal que $f\left(x_{n}\right)=y_{n}$. Usando o Lema de Urysohn, fixe $\varphi: K \longrightarrow[0,1]$ contínua tal que $\left.\varphi\right|_{\bar{W}_{2}}=1$ e $\left.\varphi\right|_{K \backslash U_{2}}=0$. Tome $\left(e_{n}\right)_{n \in \omega}$ uma sequência de funções contínuas de $K$ em [0,1], duas a duas disjuntas, tal que, para todo $n, \operatorname{supp}\left(e_{n}\right) \subset W_{2}$ e $e_{n}\left(y_{n}\right)=1$. 
Defina $T: C(K) \longrightarrow C(K)$ o operador tal que $T(g)(x)=0$, se $x \notin U_{1}$, e $T(g)(x)=$ $\varphi(f(x)) \cdot(g \circ f(x))$ se $x \in U_{1}$. Temos que $T$ está bem definida $(T(g)$ é contínua) e é linear e contínua. Além disso, temos $e_{n}\left(x_{n}\right)=0$ mas $T\left(e_{n}\right)\left(x_{n}\right)=e_{n}\left(f\left(x_{n}\right)\right)=1$. Portanto, $T$ não é um multiplicador fraco, contradizendo que $K$ é fracamente Koszmider.

Num espaço $K$ compacto, sem pontos isolados e fracamente Koszmider, o único homeomorfismo que pode ser construído sobre $K$ é a identidade, porque se existir um ponto com imagem diferente então o fato de $K$ ser Hausdorff (lembramos que aqui os compactos sempre são considerados Hausdorff) implica que existem dois abertos disjuntos homeomorfos, contradizendo o próximo resultado.

Proposição 2.15. Se K é um espaço fracamente Koszmider sem pontos isolados, então K não contém dois abertos disjuntos, não vazios e homeomorfos.

Demonstração: Sejam $K$ um espaço fracamente Koszmider, $\Omega$ e $\Omega^{\prime}$ dois abertos em $K$ não vazios disjuntos, e $\varphi: \Omega \rightarrow \Omega^{\prime}$ um homeomorfismo.

Usando normalidade do $K$, tome $W_{2}$ um aberto de $K$ tal que $\varphi(x) \in W_{2} \subset \overline{W_{2}} \subset \Omega^{\prime}$. Usando continuidade, tome $U \subset \Omega$ tal que $x \in U$ e $\varphi[U] \subset W_{2}$. Temos que $U$ é infinito, pois $K$ não tem ponto isolado, é como $\varphi$ é homeomorfismo, $\varphi[U]$ também é infinito.

Seja $\left(y_{n}\right)_{n \in \mathbb{N}}$ uma sequência relativamente discreta em $\Omega^{\prime}$. Tome $x_{n}=\varphi^{-1}\left(y_{n}\right)$.

Usando o Lema de Urysohn, fixe $f: K \rightarrow[0,1]$ contínua, tal que $\left.f\right|_{\overline{W_{2}}}=1$ e $\left.f\right|_{K \backslash \Omega^{\prime}}=0$. tome $\left(e_{n}\right)_{n \in \mathbb{N}}$ uma sequência de funções contínuas de $K$ em $[0,1]$, duas a duas disjuntas tal que, para todo $n, \operatorname{supp}\left(e_{n}\right) \subset W_{2}$ e $e_{n}\left(y_{n}\right)=1$.

Defina o operador $T: C(K) \longrightarrow C(K)$ por

$$
T(g)(x)= \begin{cases}(f \circ \varphi)(x) \cdot(g \circ \varphi)(x) & \text { se } x \in \Omega \\ 0 & \text { outro caso }\end{cases}
$$

Note $T(g)$ é contínua, portanto $T$ é bem definido, linear e contínuo. Adicionalmente, $e_{n}\left(x_{n}\right)=0$ mas $T\left(e_{n}\right)\left(x_{n}\right)=e_{n}\left(y_{n}\right)=1$. Portanto, $T$ não é multiplicador fraco, contradizendo que $K$ é fracamente Koszmider.

Finalizamos esta seção com uma condição necessária para $K$ ser fracamente Koszmider que é consequência imediata do Teorema 3.2 em [FKP13].

Proposição 2.16 (Fajardo et al., [FKP13]). Se K é fracamente Koszmider, então K não contém sequência convergente não trivial.

\subsection{Condições suficientes para $C(K)$ ter poucos operadores}

Agora, usando o Lema 2.13, fornecemos condições suficientes para um espaço compacto ser fracamente Koszmider. Essas condições também são suficientes para que alguns subespaços fechados sejam fracamente Koszmider. Precisamos primeiro provar que é possível estender famílias enumeráveis de funções definidas num subespaço fechado de $K$ preservando os suportes contidos em abertos disjuntos. 
Lema 2.17. Sejam $L \subset K$ espaços topológicos compactos e Hausdorff, $\left(f_{n}\right)_{n \in N}$ uma sequência de funções contínuas de $L$ em $[0,1]$, e $\left(V_{n}\right)_{n \in \mathbb{N}}$ uma sequência de abertos dois a dois disjuntos em $L$ tais que $\operatorname{supp}\left(f_{n}\right) \subset V_{n}$, para todo $n \in \mathbb{N}$. Então existe uma sequência $\left(g_{n}\right)_{n \in N}$ de funções contínuas de $K$ em $[0,1]$ com suportes contidos em abertos disjuntos tais que $\left.g_{n}\right|_{L}=f_{n}$, para todo $n \in N$.

Demonstração: Fixe $K, L,\left(f_{n}\right)_{n \in \mathbb{N}}$ e $\left(V_{n}\right)_{n \in \mathbb{N}}$ como na hipótese.

Mostraremos que existe uma sequência $\left(A_{n}\right)_{n \in \mathbb{N}}$ de abertos dois a dois disjuntos de $K$ tal que $\operatorname{supp}\left(f_{n}\right) \subset A_{n}$, para todo $n \in \mathbb{N}$. Para isso, fixamos abertos $V_{n}^{\prime}$ s de $K$ tais que $V_{n}=V_{n}^{\prime} \cap L$. Como $L$ é fechado em $K$ e $\operatorname{supp}\left(f_{n}\right)$ é fechado em $L$, então $\operatorname{supp}\left(f_{n}\right)$ é fechado em $K$ para todo $n \in \mathbb{N}$. Pela normalidade de $K$, existem abertos $W_{n}^{\prime}$ de $K$ - para cada $n \in \mathbb{N}$ - tais que

$$
\operatorname{supp}\left(f_{n}\right) \subset W_{n}^{\prime} \subset \overline{W_{n}^{\prime}} \subset V_{n}^{\prime} .
$$

Denotamos por $W_{n}$ a interseção $W_{n}^{\prime} \cap L$. Então $W_{n}$ é um aberto em $L$ tal que $\operatorname{supp}\left(f_{n}\right) \subset W_{n} \subset$ $\overline{W_{n}} \subset \overline{W_{n}^{\prime}} \cap L \subset V_{n}$, onde o fecho de $W_{n}$ é tomado em $L$.

Vamos definir $\left(A_{n}\right)_{n \in \mathbb{N}}$ recursivamente. Suponha definidos $\left(A_{k}\right)_{k<n}$ abertos dois a dois disjuntos de $K$ tais que $A_{k} \cap L=W_{k}$ e $A_{k} \subset V_{k}^{\prime}$, para todo $k<n$. Definimos $A_{n}=U_{n} \cap W_{n}^{\prime}$, onde

$$
U_{n}=V_{n}^{\prime} \backslash \bigcup_{k<n} \bar{A}_{k}
$$

Note que $W_{n} \subset U_{n} \cap L$. De fato, para mostrar isso é suficiente provar que $W_{n} \cap \bar{A}_{k}=\emptyset$, para todo $k<n$, o que é verdade, pois $A_{k} \subset W_{k}^{\prime} \subset V_{k}^{\prime}$, que é disjunto de $W_{n}$ e $\overline{W_{k}^{\prime}} \subset V_{k}^{\prime}$. Assim, temos $A_{n} \cap L=W_{n}$. Pela construção, fica evidente que $A_{n}$ é disjunto de $A_{k}$, para $k<n$, e $A_{n} \subset V_{n}^{\prime}$.

Para o resto da construção, fixe $n \in \mathbb{N}$. Construiremos $g_{n}$ que estende $f_{n}$ e tem suporte contido em $A_{n}$. Lembrando que $\operatorname{supp}\left(f_{n}\right)$ é também fechado em $K$, usando o Lema de Uryssohn, fixe $h_{n}: K \longrightarrow[0,1]$ contínua tal que $\left.h_{n}\right|_{\operatorname{supp}\left(f_{n}\right)}=1$ e $\left.h_{n}\right|_{K \backslash A_{n}}=0$. Usando o Teorema de Tietze, fixe $\tilde{f}_{n}$ uma extensão contínua de $f$ a $K$. Defina $g_{n}=\tilde{f}_{n} \cdot h_{n}$.

Claramente as funções da sequência $\left(g_{n}\right)_{n \in \mathbb{N}}$ são duas a duas disjuntas e têm suportes contidos em abertos disjuntos, pois $\operatorname{supp}\left(g_{n}\right) \subset A_{n}$. Agora vejamos que $\left.g_{n}\right|_{L}=f_{n}$. Se $x \in L \backslash \operatorname{supp}\left(f_{n}\right)$, então $\tilde{f}_{n}(x)=f_{n}(x)=0$ e, portanto, $g_{n}(x)=0=f_{n}(x)$. Se $x \in \operatorname{supp}\left(f_{n}\right)$, então $h(x)=1$ e, portanto, $g_{n}(x)=f_{n}(x)$, provando que $g_{n}$ estende $f_{n}$.

Frantz, em 1995, em [Fra95], demonstrou que as funções pertencentes a uma família finita de funções reais contínuas, duas a duas disjuntas, definidas num subconjunto fechado de um espaço normal $X$ podem ser estendidas a todo $X$ simultaneamente de modo que as extensões sejam contínuas e duas a duas disjuntas (ver Proposição 5, [Fra95]). Adicionalmente, Frantz provou que se $X$ é um espaço metrizável esse tipo de extensões podem ser feitas para famílias arbitrárias de funções contínuas, duas a duas disjuntas, definidas em um subconjunto fechado de $X$ (ver Proposição 6 em [Fra95]).

No mesmo artigo, Frantz afirmou que para os espaços normais ainda era possível fazer extensões contínuas que foram duas a duas disjuntas para famílias infinitas enumeráveis de funções definidas em um subconjunto fechado e que tiveram esta propriedade, mas que a prova era bastante técnica que apareceria num artigo posterior. Nós não achamos uma prova de essa afirmação em trabalhos posteriores de Frantz. Posteriormente nós conhecemos um trabalho de Barov e Dijkstra de 2002 
onde a afirmação de Frantz foi demonstrada, ver Corolário 12 em [BD02]. A prova usa a Proposição 6 de [Fra95]. Assim os resultados de [Fra95] e [BD02] implicam o Lema 2.17 mas a prova que nós apresentamos não usa as técnicas de [Fra95] e segue simplesmente da normalidade do espaço.

A prova do próximo teorema é uma leve adaptação da demonstração do Teorema 5.10 de [Faj10].

Teorema 2.18. Sejam $K$ um espaço compacto e $Q \subset K$ denso. Suponha que, dados:

(a) Uma sequência $\left(f_{n}\right)_{n \in \mathbb{N}}$ de funções contínuas de $K$ em $[0,1]$ com suportes contidos em abertos disjuntos;

(b) uma sequência relativamente discreta $\left(x_{n}\right)_{n \in \mathbb{N}}$ de pontos distintos de $Q$ tal que $f_{m}\left(x_{n}\right)=0$, para todo $n, m \in \mathbb{N}$;

(c) $\varepsilon>0$;

(d) uma sequência limitada $\left(\mu_{n}\right)_{n \in \mathbb{N}}$ de medidas regulares em $K$ tal que $\left|\int f_{n} d \mu_{n}\right|>\varepsilon$, para todo $n \in \mathbb{N}$

existem $\delta>0, b \subset a \subset \mathbb{N}$ infinitos, e funções continuas $f_{n}^{\prime}$ de $K$ em $[0,1], \operatorname{com} \operatorname{supp}\left(f_{n}^{\prime}\right) \subset \operatorname{supp}\left(f_{n}\right)$, tais que

(e) $\left|\int f_{n}^{\prime} d \mu_{n}\right|>\delta e \sum\left\{\int f_{m}^{\prime} d\left|\mu_{n}\right|: n \neq m, m \in a\right\}<\delta / 3$, para todo $n \in a$;

(f) $\left(f_{n}^{\prime}\right)_{n \in b}$ tem supremo em $C(K)$;

(g) $\overline{\left\{x_{n}: n \in b\right\}} \cap \overline{\left\{x_{n}: n \in a \backslash b\right\}} \neq \emptyset$.

Então $K$ é um espaço fracamente Koszmider. Além disso, se $L \subset K$ é fechado e $Q \cap L$ é denso em $L$, então L é fracamente Koszmider.

Demonstração: Sejam $K$ e $Q$ como acima e $L$ um subconjunto fechado de $K \operatorname{com} Q \cap L$ denso em $L$.

Supondo, por absurdo, que $L$ não é fracamente Koszmider, pelo Lema 2.13 existem $T: C(L) \rightarrow$ $C(L)$ linear e contínuo, $\varepsilon>0$, uma sequência $\left(e_{n}\right)_{n \in \mathbb{N}}$ de funções contínuas de $L$ em $[-1,1]$ com suportes contidos em abertos disjuntos, e $\left(x_{n}\right)_{n \in \mathbb{N}}$ em $L$ tais que $e_{n}\left(x_{n}\right)=0$ e $\left|T\left(e_{n}\right)\left(x_{n}\right)\right|>\varepsilon$ para infinitos $n \in \mathbb{N}$. Tomando uma subsequência se pode assumir $\left|T\left(e_{n}\right)\left(x_{n}\right)\right|>\varepsilon$ para todo $n \in \mathbb{N}$.

Pela normalidade de $K$ e a continuidade de $e_{n}$ e $T$, é possível modificar $e_{n}$ numa vizinhança aberta de $x_{n}$ de forma que as propriedades acima sejam preservadas e $e_{n}$ é 0 nessa vizinhança. Com efeito, seja $\delta$ tal que se $\left\|g-e_{n}\right\|<\delta$. Temos $\left\|T(g)-T\left(e_{n}\right)\right\|<\frac{1}{2}\left(\left|T\left(e_{n}\right)\left(x_{n}\right)\right|-\varepsilon\right)$ e, pela continuidade de $e_{n}$, existe um aberto $V_{n}$ contendo $x_{n}$ tal que $\left|e_{n}(x)\right|<\delta$, para todo $x \in V_{n}$. Usando normalidade de $K$ achamos $W_{n}$ aberto com $x_{n} \in W_{n} \subset \overline{W_{n}} \subset V_{n}$; pelo Lema de Urysohn existe $g_{n}: L \rightarrow[0,1]$ contínua com $\left.g_{n}\right|_{\bar{W}}=0$ e $\left.g_{n}\right|_{L \backslash V}=1$. Logo, $\left\|e_{n}-g_{n} e_{n}\right\|<\delta$, que, junto com $\| T\left(e_{n}\right)-T\left(e_{n} g_{n}\right)|| \geq\left|T\left(e_{n}\right)\left(x_{n}\right)\right|-\left|T\left(e_{n} g_{n}\right)\left(x_{n}\right)\right|$, implica $\left|T\left(e_{n} g_{n}\right)\left(x_{n}\right)\right|>\varepsilon$. Portanto, basta usar $g_{n} e_{n}$ no lugar de $e_{n}$.

Assim, substituindo cada $x_{n}$ por outro ponto em $Q \cap L$, podemos assumir, sem perda de generalidade, que $x_{n} \in Q \cap L$.

Indo para uma subsequência, assumimos que $x_{n} \neq x_{m}$, sempre que $m \neq n$. De fato, devido somas finitas de $e_{n}$ 's são contínuas e uniformemente limitadas. Portanto se, $x_{n}$ for constante para infinitos 
$n$ 's, então $\left|T\left(e_{n}\right)\left(x_{n}\right)\right|>\varepsilon$ implicaria $\left\|T\left(\sum_{n=1}^{N} e_{n}\right)\right\|>N \varepsilon$, para qualquer $N>1$, contradizendo que $T$ é limitado.

É possível assumir, sem perda de geralidade, que $e_{n}\left(x_{m}\right)=0$, para todos $m, n \in \mathbb{N}$. Com efeito, se existir $n_{0} \in \mathbb{N}$ tal que $e_{n_{0}}\left(x_{m}\right) \neq 0$, para todo $m \in N_{0}$, onde $N_{0}$ é um subconjunto infinito de $\mathbb{N}$, então a subsequência $\left(e_{n}\right)_{n \in N_{0} \backslash\left\{n_{0}\right\}}$ verifica $e_{n}\left(x_{m}\right)=0$, para todo $m, n \in N_{0} \backslash\left\{n_{0}\right\}$, devido ao fato das funções serem duas a dua disjuntas. Caso contrário, constrói-se por indução uma sequência escolhendo $k_{n}>k_{n-1}$ e tal que $e_{m}\left(x_{k_{n}}\right)=0$, para todo $m \leq k_{n}$.

Também, $e_{n}$ se pode assumir com imagem em $[0,1]$, para todo $n \in \mathbb{N}$, considerando $\max \left(e_{n}, 0\right)-$ $\min \left(e_{n}, 0\right)$ no lugar de $e_{n}$.

Agora, seja $\delta_{x_{n}}$ a medida de Dirac concentrada em $x_{n}$. Usando o Teorema de Representação de Riesz, definimos $\mu_{n}=T^{*}\left(\delta_{x_{n}}\right)$, i.e., $T(f)\left(x_{n}\right)=\int f d \mu_{n}$ para todo $f \in C(L)$. Portanto

$$
\left|\int e_{n} d \mu_{n}\right|>\varepsilon
$$

para todo $n \in \mathbb{N}$. Note que $\left(\mu_{n}\right)_{n \in \mathbb{N}}$ é limitado porque $T$ é limitado.

Usando o Lema 2.17, podemos tomar $f_{n}$ como extensões contínuas de $e_{n}$ a $K$ preservando as imagens em $[0,1]$ e suportes contidos em abertos disjuntos. Para cada $n \in \mathbb{N}$ consideramos $\mu_{n}$ como a mesma medida em $K$, i.e, substituímos $\mu_{n}$ por $\widetilde{\mu}_{n}$ definida como $\widetilde{\mu}_{n}(E)=\mu_{n}(E \cap L)$, onde $E$ é um conjunto boreliano em $K$.

Note que $\left(f_{n}\right)_{n \in \mathbb{N}},\left(x_{n}\right)_{n \in \mathbb{N}},\left(\mu_{n}\right)_{n \in \mathbb{N}}$, e $\varepsilon$ verificam (a)-(d) acima. Então existe $\delta>0, b \subset a \subset \mathbb{N}$ infinito e funções contínuas $f_{n}^{\prime}$ de $K$ em $[0,1]$, com $\operatorname{supp}\left(f_{n}^{\prime}\right) \subset \operatorname{supp}\left(f_{n}\right)$, satisfazendo (e)-(g).

Agora vejamos que é possível supor

$$
\int \sup \left\{f_{m}^{\prime}: m \in b\right\} d \mu_{n}=\int \sum_{m \in b} f_{m}^{\prime} d \mu_{n}
$$

para todo $n \in \mathbb{N}$. De fato, consideremos $\left(N_{\xi}\right)_{\xi<\omega_{1}}$ uma sequência de subconjuntos infinitos de $\mathbb{N}$ tal que $\left|N_{\xi} \cap N_{\eta}\right|<\infty$, para todo $\xi \neq \eta$ (isso é possível identificando $\mathbb{N}$ com $\mathbb{Q}$ e $\mathbb{R}$ com $2^{\omega}$ e tomando $N_{\xi}$ como uma sequência de racionais convergindo a $\xi$ ). Para cada $\xi \in \omega_{1}$ tomamos $b_{\xi} \subset a_{\xi} \subset N_{\xi}$ como $a$ e $b$ acima. Para $n \in b_{\xi}$ denotamos $f_{n}^{\xi}=f_{n}^{\prime}$. Existe $\xi<\omega_{1}$ tal que $a=a_{\xi}, b=b_{\xi}$ e $f_{n}^{\prime}=f_{n}^{\xi}$ satisfazem as propriedades (e)-(g). A seguinte afirmação determina como escolher $\xi$.

Afirmação 2.1. Existe $\xi<\omega_{1}$ tal que $\int\left[\sup \left\{f_{m}^{\xi}: m \in b_{\xi}\right\}-\sum_{m \in b_{\xi}} f_{m}^{\xi}\right] d \mu_{n}=0$, para todo $n \in \mathbb{N}$.

Começamos a prova da afirmação introduzindo alguma notação. Para todo $\xi<\omega_{1}$ e $c \subset b_{\xi}$ definimos $f_{c}^{\xi}=\sup \left\{f_{m}^{\xi}: m \in c\right\}-\sum_{m \in c} f_{m}^{\xi}$ sempre que o supremo existir.

Note que, para qualquer $F \subset b_{\xi}$ finito, acontece

$$
\sup \left\{f_{m}^{\xi}: m \in b_{\xi}\right\}=\sup \left\{f_{m}^{\xi}: m \in b_{\xi} \backslash F\right\}+\sum_{m \in F} f_{m}^{\xi}
$$

e, assim, $f_{b_{\xi} \backslash F}^{\xi}=f_{b_{\xi}}^{\xi}$. Em particular, $f_{b_{\xi} \backslash b_{\xi^{\prime}}}^{\xi}=f_{b_{\xi}}^{\xi}$, para todo $\xi \neq \xi^{\prime}$ em $\omega_{1}$, devido a $b_{\xi} \cap b_{\xi^{\prime}}$ ser finito.

Agora, sejam $\xi$ e $\xi^{\prime}$ ordinais diferentes em $\omega_{1}$. Tome $g=\sup \left\{f_{n}^{\xi}: n \in b_{\xi} \backslash b_{\xi^{\prime}}\right\}$ e $h=\sup \left\{f_{n}^{\xi^{\prime}}:\right.$ $\left.n \in b_{\xi^{\prime}} \backslash b_{\xi}\right\}$. Como $\operatorname{supp}\left(f_{n}^{\eta}\right) \subset \operatorname{supp}\left(f_{n}\right)$, para todo $n \in \mathbb{N}$ e $\eta<\omega_{1}$, tem-se $f_{n}^{\xi} \cdot f_{m}^{\xi^{\prime}}=0$ para todo 
$n \neq m$. Vejamos que $g \cdot h=0$.

Assumindo $g(x)>0$ e $h(x)>0$ para algum $x \in K$. Então existe uma vizinhança aberta $V$ de $x$ tal que $\left.g\right|_{V}>0$ e $\left.h\right|_{V}>0$. Logo, existem $n_{0} \in b_{\xi} \backslash b_{\xi^{\prime}}$ e $x_{0} \in V$ tais que $f_{n_{0}}^{\xi}\left(x_{0}\right)>0$. Portanto, existe uma vizinhança aberta $V_{0} \subset V$ de $x_{0}$ tal que $f_{n_{0}}^{\xi}(x)>0$, para todo $x \in V_{0}$. Por outro lado, como $\left.h\right|_{V_{0}}>0$, existe $n_{1} \in b_{\xi^{\prime}} \backslash b_{\xi}$ e $x_{1} \in V_{0}$ tal que $f_{n_{1}}^{\xi^{\prime}}\left(x_{1}\right)>0$, contradizendo que $f_{n_{1}}^{\xi^{\prime}}$ e $f_{n_{0}}^{\xi}$ são disjuntas.

Como $f_{b_{\xi}}^{\xi}=f_{b_{\xi} \backslash b_{\xi^{\prime}}}^{\xi} \leq g$ e $f_{b_{\xi^{\prime}}}^{\xi^{\prime}}=f_{b_{\xi^{\prime}} \backslash b_{\xi}}^{\xi^{\prime}} \leq h$, temos $f_{b_{\xi}}^{\xi} \cdot f_{b_{\xi^{\prime}}}^{\xi^{\prime}}=0$ para todo $\xi \neq \xi^{\prime}$. Então, como $\mu_{n}$ é limitado, o conjunto $A_{n, k}=\left\{\xi<\omega_{1}: \int f_{b_{\xi}}^{\xi} d\left|\mu_{n}\right|>\frac{1}{k}\right\}$ é finito, para todo $n \in \mathbb{N}$ e $k \in \mathbb{N}^{*}$. Assim, o conjunto $A=\left\{\xi<\omega_{1}: \exists n \in \mathbb{N}\left(\int f_{b_{\xi}}^{\xi} d\left|\mu_{n}\right| \neq 0\right)\right\}$ é enumerável. Consequentemente, se tomamos $\xi \in \omega_{1} \backslash A$ temos $\int f_{b_{\xi}}^{\xi} d\left|\mu_{n}\right|=0$ e, em particular, $\int f_{b_{\xi}}^{\xi} d \mu_{n}=0$, que é a condição estabelecida na afirmação.

Tomando $\xi$ satifazendo a condição do enunciado da afirmação, fixamos $a=a_{\xi}, b=b_{\xi}, f_{n}^{\prime}=f_{n}^{\xi}$ e $f=\sup \left\{f_{n}^{\prime}: n \in b\right\}$. Se $n \in b$ então

$$
\begin{aligned}
\left|T\left(\left.f\right|_{L}\right)\left(x_{n}\right)\right| & =\left|\int_{K} f_{n}^{\prime} d \mu_{n}\right|=\left|\int f_{n}^{\prime} d \mu_{n}+\int \sum\left\{f_{m}^{\prime}: m \neq n, m \in b\right\} d \mu_{n}\right| \\
& \geq \delta-\delta / 3=2 \delta / 3
\end{aligned}
$$

Alem disso, se $n \in a \backslash b$ então $\left|T\left(\left.f\right|_{L}\right)\left(x_{n}\right)\right|=\left|\int \sum\left\{f_{m}^{\prime}: m \in b\right\} d \mu_{n}\right| \leqslant \delta / 3$. Pela continuidade de $T\left(\left.f\right|_{L}\right)$ concluímos que $\overline{\left\{x_{n}: n \in b\right\}} \cap \overline{\left\{x_{n}: n \in a \backslash b\right\}}=\emptyset$ o que contradiz (g).

Note que, se $Q$ é o próprio $K$ - ou seja, se no item (b) das hipóteses do teorema podemos tomar qualquer sequência $\left(x_{n}\right)_{n \in \mathbb{N}}$ em $K$ - o Teorema 2.18 fornece uma condição suficiente para $K$ ser hereditariamente fracamente Koszmider. Isto é, todo subespaço fechado e infinito de $K$ é fracamente Koszmider. Esse é o caso da construção feita em [Faj12].

O próximo teorema é uma versão simplificada - quando aplicamos o Lema de Rosenthal - do Teorema 2.18 acima e uma adaptação do Lema 5.2 de [Kos04].

Teorema 2.19. Sejam $K$ um espaço compacto e $Q \subset K$ denso. Suponha que, dados

(a) Uma sequência $\left(f_{n}\right)_{n \in \mathbb{N}}$ de funções contínuas de $K$ em $[0,1]$ com suportes contidos em abertos dois a dois disjuntos,

(b) Uma sequência relativamente discreta $\left(x_{n}\right)_{n \in \mathbb{N}}$ de pontos diferentes de $Q$ tais que $f_{m}\left(x_{n}\right)=0$, para todo $m, n \in \mathbb{N}$;

existe um subconjunto infinito $b$ de $\mathbb{N}$ tal que

(c) $\left(f_{n}\right)_{n \in b}$ tem supremo em $C(K)$,

(d) $\overline{\left\{x_{n}: n \in b\right\}} \cap \overline{\left\{x_{n}: n \in \mathbb{N} \backslash b\right\}} \neq \emptyset$.

Então $K$ é um espaço fracamente Koszmider. Além disso, se $L \subset K$ é fechado e $Q \cap L$ é denso em L, então L é um espaço fracamente Koszmider. 
Demonstração: Suponha, por absurdo, que $T: C(L) \rightarrow C(L)$ não é multiplicador fraco. Como na prova do Teorema 2.18, pode-se supor que existem funções contínuas $e_{n}: L \rightarrow[0,1]$ com suportes contidos em abertos disjuntos, $x_{n} \in Q \cap L$, e $\varepsilon>0$ tal que

$$
\left|T\left(e_{n}\right)\left(x_{n}\right)\right|>\varepsilon,
$$

para todo $n \in \mathbb{N}$.

Tomando $\mu_{n}=T^{*}\left(\delta_{x_{n}}\right)$ em $L$, pelo Teorema de Representação de Riesz (e por abuso de notação, escrevendo o funcional com o mesmo simbolo da medida que representa), temos

$$
\left|\int e_{n} d \mu_{n}\right|=\left|T^{*}\left(\delta_{x_{n}}\right)\left(e_{n}\right)\right|=\left|\delta_{x_{n}}(T)\left(e_{n}\right)\right|>\varepsilon,
$$

para todo $n \in \mathbb{N}$. Note que $\left(\mu_{n}\right)_{n \in N}$ é limitado, porque $T$ é limitado.

Considerando $\left(\nu_{n}\right)_{n \in \mathbb{N}}$ onde $\nu_{n}$ é uma medida sobre $\mathcal{P}(\mathbb{N})$ definida por $\nu_{n}(\{m\})=\int f_{m} d \mu_{n}$. Note que $\left(\nu_{n}\right)_{n \in \mathbb{N}}$ é limitada e $(\{m\})_{m \in \mathbb{N}}$ é duas a duas disjunta, então pelo Lema de Rosenthal (ver Lema 1.12, Capítulo 1) existe $N^{\prime} \subset \mathbb{N}$ infinito tal que

$$
\sum\left\{\left|\int e_{m} d \mu_{n}\right|: n \neq m, m \in N^{\prime}\right\}<\varepsilon / 3
$$

Renumerando os índices, podemos assumir que a última desigualdade é verdadeira para $\mathbb{N}$, no lugar de $N^{\prime}$.

Agora nós usamos o Lema 2.17 para achar, para cada $n \in \mathbb{N}$, extensões $f_{n}$ a $K$ das funções $e_{n}$ de forma que continuam sendo duas a duas disjuntas e com imagens em $[0,1]$. Denotamos por $\tilde{\mu}_{n}$ as medidas em $K$ definidas por $\tilde{\mu}_{n}(E \cap L)$, onde $E$ é um conjunto boreliano em $K$. Logo, $\int_{K} f_{m} d \tilde{\mu}_{n}=\int_{L} e_{m} d \mu_{n}$. Por um abuso de notação, escreveremos $\mu_{n}$ no lugar de $\tilde{\mu}_{n}$.

Argumentando como na prova da Afirmação 2.1 do Teorema 2.18, para $f_{m}$ no lugar de $f_{m}^{\prime}$ e $a=\mathbb{N}$, assumimos que

$$
\int \sup \left\{f_{m}: m \in b\right\} d \mu_{n}=\int \sum_{m \in b} f_{m} d \mu_{n},
$$

para todo $n \in \mathbb{N}$ e algum $b \subset \mathbb{N}$ infinito.

Como na prova do Teorema 2.18, seja $f=\sup \left\{f_{n}: n \in b\right\}$. Se $n \in b$, então

$$
\left|T\left(\left.f\right|_{L}\right)\left(x_{n}\right)\right|=\left|\int f_{n} d \mu_{n}+\int \sum\left\{f_{m}: m \neq n, m \in b\right\} d \mu_{n}\right|>2 \varepsilon / 3
$$

Por outro lado, se $n \in \mathbb{N} \backslash b$, então $T\left(\left.f\right|_{L}\right)\left(x_{n}\right) \leq \varepsilon / 3$. Logo, pela continuidade de $T\left(\left.f\right|_{L}\right)$, temos $\overline{\left\{x_{n}: n \in \mathbb{N} \backslash b\right\}} \cap \overline{\left\{x_{n}: n \in b\right\}}=\emptyset$, o que contradiz (d).

Note que se $Q$ é um subespaço denso de um espaço compacto $K$, então, para todo aberto $V \subset K, Q \cap V$ é denso em $\bar{V}$. Portanto se $K$ e $Q$ verificam as condições do Teorema 2.19, então $\bar{V}$ é fracamente Koszmider. Se, além disso, $K$ não possui abertos-borboleta então, pelo Lema 2.10. para todo subconjunto aberto $V$ de $K$ temos que $\bar{V}$ não contém abertos-borboleta e assim, $\bar{V}$ é um espaço de Koszmider para todo aberto $V \subset K$. A construção que faremos no Capítulo 6 satisfaz 
essa propriedade.

No Teorema 3.8, demonstraremos que $L$ é fracamente Koszmider sempre que $C(L)$ é complementado em $C(K)$ e $K$ é fracamente Koszmider. Em particular, se $L$ é um subespaço aberto e fechado de um espaço fracamente Koszmider, então ele também é fracamente Koszmider. 


\section{Capítulo 3}

\section{Subespaços complementados de espaços com poucos operadores}

Um espaço $C(K)$ com poucos operadores possui, em particular, poucas projeções. Consequentemente, um aspecto interessante da geometria destes espaços é a sua rigidez em termos da decomposição em subespaços complementados. No caso que $K$ é Koszmider e conexo, de fato, as únicas projeções em $\mathcal{L}(C(K))$ são as que a dimensão do núcleo ou da imagem é finita. Neste capítulo estudaremos relações entre espaços indecomponíveis e subespaços de espaços $C(K)$ com poucos operadores.

\subsection{Espaços $C(K)$ indecomponíveis e espaços com poucos opera- dores}

No caso em que $K \backslash F$ é conexo, para todo $F$ finito, $C(K)$ ter poucos operadores implica que suas únicas decomposições são triviais (uma das parcelas tem dimensão finita), conforme provado por Koszmider, no teorema que enunciamos a seguir. Mas mesmo no caso em que $K$ é totalmente desconexo - o que implica que $C(K)$ não é indecomponível-, se $C(K)$ tem poucos operadores há várias restrições sobre os seus subespaços complementados.

Teorema 3.1 (Teorema 2.5 [Kos04].). Seja $K$ um espaço fracamente Koszmider tal que $K \backslash F$ é conexo para todo $F \subset K$ finito. Então, todas as projeções em $C(K)$ são da forma $I+S$ onde $S$ é um operador cuja imagem tem dimensão finita. Equivalentemente, todo subespaço complementado de $C(K)$ tem dimensão finita ou codimensão finita, isto é, $C(K)$ é um espaço de Banach indecomponivel.

A condição $K \backslash F$ é conexo é necessária para um espaço compacto conexo $K$ ser Koszmider.

Proposição 3.2 (Corolário 5.3 [Sch08a].). Seja K um espaço Koszmider conexo. Então para qualquer subconjunto finito $F$ de $K$ o espaço $K \backslash F$ é conexo.

O próximo corolário é imediato do fato que todo espaço Koszmider é fracamente Koszmider (ver Proposição 2.4) e da proposição e o teorema anterior. Também há uma prova direta desse corolário em [Faj09].

Corolário 3.3. Seja K um espaço compacto e conexo. Se K é Koszmider, então $C(K)$ é indecomponivel. 
É conhecido, pelo Corolário 1.10, que uma condição necessária para que um subespaço de dimensão infinita $X$ de $C(K)$ seja complementado é que $X$ contenha um subespaço isomorfo a $c_{0}$. Para obter condições adicionais no caso que $C(K)$ tem poucos operadores nós usamos a caracterização de espaços fracamente Koszmider dada por Schlackow em [Sch08a]. Denotamos por $\mathcal{L}(X)$ o espaço de todos os operadores lineares e contínuos em $X$, e por $\mathcal{W}(X)$ o espaço de todos os operadores (lineares e contínuos) fracamente compactos em $X$.

Teorema 3.4 ([Sch08a], Teorema 4.5.). Um espaço compacto $K$ é fracamente Koszmider se, e somente se, $\mathcal{L}(C(K)) / \mathcal{W}(C(K))$ é comutativo, i.e., se $T \circ S-S \circ T$ é fracamente compacto, para todo operador $T$ e $S$ em $\mathcal{L}(C(K))$.

Como consequência do teorema anterior, a propriedade de ser fracamente Koszmider é invariante por isomorfismos de espaços $C(K)$ 's.

Teorema 3.5 ([Sch08a], Teorema 4.1 (i).). Se K é fracamente Koszmider e $C(L)$ é isomorfo a $C(K)$, então L é fracamente Koszmider.

O mesmo resultado não acontece para espaços Koszmider no lugar de fracamente Koszmider.

Teorema 3.6 ([Sch08a], Proposição 4.10. ). Para todo espaço Koszmider K existe um espaço não Koszmider $L$ tal que $C(K)$ é isomorfo a $C(L)$.

Os dois últimos teoremas permitem concluir que nos espaços $C(K)$ a propriedade de ser indecomponível não implica que $K$ é Koszmider. Lembre-se que se $P \in \mathcal{L}(C(L))$ é uma projeção e $T$ é um isomorfismo de $C(K)$ sobre $C(L)$ então $T^{-1} \circ P \circ T$ é uma projeção em $\mathcal{L}(C(K))$.

Corolário 3.7. Existe um espaço indecomponível $C(L)$ tal que $L$ não é um espaço Koszmider.

Demonstração: Usando o resultado de [Kos04] ou [Ple04] junto com a Proposição 3.2, tome $K$ um espaço fracamente Koszmider tal que $C(K)$ é indecomponível. Seja $L$ como no Teorema 3.6. Como $C(L)$ é isomorfo ao $C(K)$, então $C(L)$ é indecomponível.

Ainda não se sabe se $C(K)$ indecomponível implica $K$ ser fracamente Koszmider.

\subsection{Subsespaços complementados de espaços $C(K)$ com $K$ fraca- mente Koszmider}

O nosso próximo resultado estuda os subespaços complementados da forma $C(L)$ de um espaço $C(K)$ com $K$ fracamente Koszmider.

Teorema 3.8. Se $K$ é um espaço fracamente Koszmider e $C(L)$ é isomorfo a um subespaço complementado de $C(K)$ então, L é fracamente Koszmider.

Demonstração: Suponha que $K$ é fracamente Koszmider e $P: C(K) \rightarrow C(K)$ é uma projeção sobre $C(L)$. Vejamos que $L$ é fracamente Koszmider. Pelo Teorema 3.4, é suficiente provar que $T \circ S-S \circ T$ é fracamente compacto, para todos $T, S \in \mathcal{L}(C(L))$. 
Para $T, S \in \mathcal{L}(C(L))$, defina $T^{\prime}, S^{\prime} \in \mathcal{L}(C(K))$ como

$$
T^{\prime}:=T \circ P \text { e } \quad S^{\prime}:=S \circ P .
$$

Pelo Teorema 3.4, $T^{\prime} \circ S^{\prime}-S^{\prime} \circ T^{\prime}$ é fracamente compacto. Portanto sua restrição a $C(L)$, que é $T \circ S-S \circ T$, é fracamente compacta.

Koszmider, assumindo o Axioma de Martin ou CH em [Kos05] apresentou um exemplo de um espaço compacto separável $K$ tal que todos os operadores de $C(K)$ são da forma $\lambda I+S$, onde $\lambda \in \mathbb{R}$ e $S$ tem imagem contida em $c_{0}$. Tal espaço tem a propriedade de que as únicas decomposições $C(K)=X \oplus Y$ em dois subespaços complementados de dimensão infinita são tais que $X \approx c_{0} \mathrm{e}$ $Y \approx C(K)$ ou $X \approx C(K)$ e $Y \approx c_{0}$. Em particular, este é um exemplo de um espaço que tem "poucos operadores" no sentido de Shelah e Steprāns ([SS88]) e tem um subespaço complementado $Z$ isomorfo a $Z \oplus Z$. Vejamos que esta propriedade não acontece nos espaços $C(K)$ com poucos operadores.

Teorema 3.9. Se K é um espaço fracamente Koszmider, então qualquer subespaço complementado de $C(K)$ (incluindo ele próprio) não é isomorfo a $X \oplus X$, para $X$ espaço de Banach de dimensão infinita.

Demonstração: Suponhamos que $C(K)$ é isomorfo a $X \oplus X \oplus Y$, onde $X, Y$ são espaços de Banach e $X$ tem dimensão infinita. Compondo com um isomorfismo, é possível assumir, sem perda de generalidade, que $C(K)=X \oplus X \oplus Y$.

Definimos $T: C(K) \rightarrow C(K)$ por $T(x, y, z)=(y, x, z)$ e $S: C(K) \rightarrow C(K)$ por $S(x, y, z)=$ $(-y, x, z)$. Note que $T \circ S(x, y, z)=(x,-y, z) \mathrm{e}$

$$
S \circ T(x, y, z)=(-x, y, z)
$$

Agora note que

$$
(T \circ S-S \circ T)(x, y, z)=(2 x,-2 y, 0)
$$

e, portanto, a restrição de $T \circ S-S \circ T$ ao subespaço $X$ é um isomorfismo, logo, não é um operador estritamente singular. Como os operadores em $C(K)$ são estritamente singulares se, e somente se, são fracamente compactos (ver, por exemplo, Teoremas 5.5.1 e 5.5.3 em [AK06]), então $T \circ S-S \circ T$ não é fracamente compacto, e portanto, o Teorema 3.4 implica que $K$ não é fracamente Koszmider.

Lembramos que um espaço de Banach é dito de Grothendieck se toda sequência em $X^{*}$ convergente na topologia fraca* também converge (ao mesmo limite) na topologia fraca. Ver, por exemplo, [DU77]. Schachermayer, em [Sch82], demonstrou que em $C(K)$ os espaços de Grothendieck são justamente os que não contém cópia complementada de $c_{0}$.

Do teorema anterior se pode deduzir imediatamente o fato demonstrado em [Kos04], que se $K$ é fracamente Koszmider, então $C(K)$ não contém $c_{0}$ complementado, isto é, $C(K)$ é um espaço de Grothendieck. 


\subsection{Espaços conexos fracamente Koszmider}

Considerando o Teorema 3.1 e a Proposição 3.2, uma pergunta natural é se a hipótese da conexidade para $K \backslash F$, para todo $F \subset K$ finito, é realmente necessária para obter a indecomponibilidade de $C(K)$, para $K$ fracamente Koszmider. Respondemos afirmativamente a essa questão, usando uma adaptação da construção apresentada em [Sch08a]. Usaremos também o seguinte teorema que é o caso particular do Teorema 18 de [Faj10], aplicando o Lema 16 do mesmo artigo.

Teorema 3.10 (Teorema 18, Lema 16 [Faj10]). Existe uma família $\left\{K_{\xi}: \xi<2^{\omega}\right\}$ de espaços compactos conexos fracamente Koszmider tal que $C\left(K_{\xi}\right)$ é indecomponivel, para todo $\xi<2^{\omega}$, e todo operador de $C\left(K_{\xi}\right)$ em $C\left(K_{\eta}\right)$ é fracamente compacto, para todos $\xi, \eta<2^{\omega}$ tais que $\xi \neq \eta$.

Apesar de no enunciado original do teorema anterior em [Faj10] não é explicitado que os $K_{\xi}$ são fracamente Koszmider, tal propriedade é provada na demonstração e usada para provar a indecomponibilidade de $C\left(K_{\xi}\right)$.

Observamos que se $K_{1}, K_{2}, L_{1}, L_{2}$ são compactos e $\varphi_{i}$ é um homeomorfismo de $K_{i}$ sobre $L_{i}$, para $i \in\{1,2\}$, e $T: C\left(L_{1}\right) \rightarrow C\left(L_{2}\right)$ é um operador linear não fracamente compacto, então $I_{2}^{-1} \circ T \circ I_{1}: C\left(K_{1}\right) \rightarrow C\left(K_{2}\right)$ não é fracamente compacto, onde $I_{i}$ é o isomorfismo de $C\left(K_{i}\right)$ sobre $C\left(L_{i}\right)$ definido pelo homeomorfismo $\varphi_{i}$, isto é, $I_{i}(\cdot)=(\cdot) \circ \varphi_{i}^{-1}$. Com efeito, pelo Teorema 1.15 existem $\varepsilon>0$ e uma sequência limitada $\left(f_{n}\right)_{n \in \mathbb{N}}$ de funções de $L_{1}$ em $\mathbb{R}$, duas a duas disjuntas, tais que $\left\|T\left(f_{n}\right)\right\|>\varepsilon$. Logo, $\left(f_{n} \circ \varphi_{1}\right)_{n \in \mathbb{N}}$ é limitada de funções duas a duas disjuntas e $\| I_{1} \circ T \circ$ $I_{2}^{-1}\left(f_{n} \circ \varphi_{1}\right)\|=\| T\left(f_{n}\right) \circ \varphi_{2}\|=\| T\left(f_{n}\right) \|>\varepsilon$.

Teorema 3.11. Existe um espaço compacto, conexo e fracamente Koszmider $K$ tal que $C(K)$ é decomponível. Em particular $K$ não é um espaço de Koszmider.

Demonstração: Usando o Teorema 3.10, fixe $K_{1}$ e $K_{2}$ espaços compactos, conexos e fracamente Koszmider tais que todo operador $T: C\left(K_{1}\right) \rightarrow C\left(K_{2}\right)$ é fracamente compacto. Como $K_{i}$ é homeomorfo ao subespaço $K_{i} \times\{i-1\}$ de $K_{i} \times[0,1]$ para $i \in\{1,2\}$, podemos supor que $K_{1} \cap K_{2}=\emptyset$.

Fixando $y_{1} \in K_{1}$ e $y_{2} \in K_{2}$, definimos uma relação de equivalência $\sim$ em $K_{1} \cup K_{2}$ tomando $x \sim y$ se, e somente se, $x=y$ ou $\{x, y\}=\left\{y_{1}, y_{2}\right\}$.

Tome $K$ o espaço $K_{1} \cup K_{2} / \sim$ munido da topologia quociente, com $K_{1} \cup K_{2}$ munido da topologia da união disjunta. Note que, pela compacidade de $K_{1}$ e $K_{2}$, o espaço $K$ é compacto. Mais ainda, $K_{1}$ e $K_{2}$ são homeomorfos a suas respectivas imagens por $x \rightarrow[x]$ em $K$.

Sejam $X:=\left\{f \in C(K): f\left[K_{2}\right]=\left\{f\left(\left[y_{1}\right]\right)\right\}\right\}$ e $Y:=\left\{f \in C(K): f\left[K_{1}\right]=0\right\}$. Para todo $j \in\{1,2\}$ fixo e $f \in C\left(K_{j}\right)$, definimos $\bar{f} \in C(K)$ por

$$
\bar{f}([x])=\left\{\begin{array}{lll}
f(x), & \text { se } & {[x] \in K_{j}} \\
f\left(y_{j}\right), & \text { se } & {[x] \in K \backslash K_{j} .}
\end{array}\right.
$$

Note que $f=\overline{\left.f\right|_{K_{1}}}+\overline{\left.f\right|_{K_{2}}-f\left(\left[y_{2}\right]\right)}$, para todo $f \in C(K)$. Logo, $C(K)=X+Y$.

Como $\left[y_{1}\right]=\left[y_{2}\right] \in K_{1} \cap K_{2}$, se $f \in X \cap Y$ então $f\left(\left[y_{1}\right]\right)=0=f\left(\left[y_{2}\right]\right)$, logo $f=0$. Assim, concluímos que $C(K)=X \oplus Y$ e, como os espaços $X$ e $Y$ são de dimensão infinita, $C(K)$ é indecomponível.

Agora vejamos que $K$ é um espaço fracamente Koszmider. Suponhamos que $T: C(K) \rightarrow C(K)$ não é um multiplicador fraco. Sejam $\left(e_{n}\right)_{n \in \mathbb{N}}$ uma sequência limitada de funções em $C(K)$, duas a 
duas disjuntas, $\left(x_{n}\right)_{n \in \mathbb{N}}$ uma sequência em $K$, e $\varepsilon>0$ tais que $e_{n}\left(x_{n}\right)=0$ e $\left|T\left(e_{n}\right)\left(x_{n}\right)\right|>\varepsilon$, para todo $n \in \mathbb{N}$.

Tomando subsequências de $\left(e_{n}\right)_{n \in \mathbb{N}}$ e $\left(x_{n}\right)_{n \in \mathbb{N}}$, podemos supor que $e_{n}\left(\left[y_{1}\right]\right)=0$ para todo $n$ e $x_{n} \in K_{j}$ para algum $j \in\{1,2\}$. com efeito, existe $j$ tal que $x_{n} \in K_{j}$ para infinitos $n$ 's. Se, para algum $n_{0}, e_{n_{0}}\left(y_{1}\right) \neq 0$ e $x_{n_{0}} \in K_{j}$, consideramos $\left\{n \in \mathbb{N}: x_{n} \in K_{j}, n \neq n_{0}\right\}$ no lugar de $\mathbb{N}$.

Assumimos, sem perda de generalidade, que $j=1$. Para cada par $i, k \in\{1,2\}$, definimos $T_{i, k}: C\left(K_{i}\right) \rightarrow C\left(K_{k}\right)$ por $T_{i, k}(f)=\left.T(\bar{f})\right|_{K_{k}}$. Como $e\left(\left[y_{1}\right]\right)=0$ então $e_{n}=\overline{\left.e_{n}\right|_{K_{1}}}+\overline{\left.e_{n}\right|_{K_{2}}}$. Logo:

$$
\begin{aligned}
T\left(e_{n}\right)\left(x_{n}\right) & =T\left(\overline{\left.e_{n}\right|_{K_{1}}}\right)\left(x_{n}\right)+T\left(\overline{\left.e_{n}\right|_{K_{2}}}\right)\left(x_{n}\right) \\
& =T_{1,1}\left(\left.e_{n}\right|_{K_{1}}\right)\left(x_{n}\right)+T_{2,1}\left(\left.e_{n}\right|_{K_{2}}\right)\left(x_{n}\right) .
\end{aligned}
$$

Pela escolha dos $K_{j}$ temos que $T_{2,1}$ é fracamente compacto. Logo, pelo Teorema 1.15, temos que $\lim _{n \rightarrow \infty} T_{2,1}\left(\left.e_{n}\right|_{K_{2}}\right)=0$ e, portanto, $T_{2,1}\left(\left.e_{n}\right|_{K_{2}}\right)\left(x_{n}\right)$ converge a zero. Consequentemente, $\left|T_{1,1}\left(e_{n} \mid K_{1}\right)\left(x_{n}\right)\right|>\varepsilon$ para infinitos $n$ 's, contradizendo o fato que $K_{1}$ é fracamente Koszmider.

Lembre-se que para $X, Y$ espaços de Banach, e $T: X \rightarrow Y$ um isomorfismo topológico, se $M$ é um subespaço complementado de $X$, então $T(M)$ é um subespaço complementado de $Y$. Ou seja, a noção de subespaço complementado é invariante sob isomorfismo topológico.

Lembramos que um espaço de Banach $X$ é primário se sempre que $X \approx Y \oplus Z$ acontece $X \approx Y$ ou $X \approx Z$, (ver por exemplo [AK06]). Um exemplo de um espaço de Banach com está propriedade é o espaço $\ell_{\infty}$, este espaço não é isomorfo a nenhum de seus subespaços complementados de dimensão infinita (i.e é primo, esta propriedade é mais forte que ser primário), para uma prova indicamos [AK06]. Finalizamos este capítulo nomeando rapidamente algumas propriedades de $K$ para os quais espaços da forma $C(K)$ são ou não primários.

Lindestrauss e Pełczynski em [LP71] provaram que $C([0,1])$ é primário. Pelo Teorema de Miljutin (veja [AK06]), qualquer $C(K)$ com $K$ compacto métrico não enumerável é isomorfo a $C([0,1])$. Assim, $C(K)$ é primário para $K$ metrizável, compacto e não enumerável. Além disso, D.E. Alspach e Y. Bejamini, em [AB77], demonstraram que $C(K)$ é primário se $K$ é enumerável e compacto.

Koszmider, em [Kos04], demonstrou que, se um compacto $K$ é fracamente Koszmider, então dois subespaços de codimensão infinita de $C(K)$ são isomorfos se, e somente se tem a mesma codimensão. Em particular, $C(K)$ não é isomorfo a nenhum de seus hiperplanos e não é primário. Desconhecemos outros exemplos de espaços $C(K)$ não primários em ZFC.

Drewnowski e Roberts, em 1991, em [DR91], demonstraram, assumindo CH, que o espaço $\ell_{\infty} / c_{0}$ é primário. Segundo Brech e Koszmider, em [BK14], permanece em aberto se o resultado de Drewnowski e Roberts é verdadeiro em ZFC. Como o espaço $C(\beta \mathbb{N} \backslash \mathbb{N})$, onde $\beta \mathbb{N}$ é a compactificação de Stone-Cech, é isometricamente isomorfo a $\ell_{\infty} / c_{0}$ esse é um exemplo de um espaço $C(K)$ não primário em $\mathrm{ZFC}+\mathrm{CH}$ e que desconhecemos, em $\mathrm{ZFC}$, se não é primário. 


\section{Capítulo 4}

\section{Conjunto dos Operadores Não Multiplicadores Fracos}

Neste capítulo estudaremos o conjunto dos operadores não multiplicadores fracos de espaços $C(K)$, que denotaremos (pelo nome em inglês) por $\operatorname{NWM}(C(K))$, quando $K$ não é fracamente Koszmider. Especificamente, pretendemos investigar o tamanho desse conjunto através das estruturas lineares que ele pode conter.

Em [FKP13] foi demonstrado que se existir um operador em $C(K)$ que não é multiplicador fraco então, existe um espaço vetorial de dimensão infinita contido em $\operatorname{NWM}(C(K)) \cup\{0\}$. Mais ainda, esse espaço é subespaço fechado de $\mathcal{L}(C(K))$.

Na próxima seção apresentamos os conceitos de lineabilidade e espaçabilidade que usaremos para estudar o conjunto $\operatorname{NWM}(C(K))$, apresentando alguns resultados obtidos por outros autores. Na Seção 2 apresentamos o nosso resultado principal (Teorema 4.5) de este capítulo.

\subsection{Lineabilidade e espaçabilidade do conjunto dos operadores não multiplicadores fracos de um espaço $C(K)$}

As noções de espaçabilidade e lineabilidade foram primeiro introduzidas por Vladimir Gurariy, em [GQ04] e [AGS05]. Contudo, ele mesmo e outros autores tinham trabalhado anteriormente achando grandes estruturas algébricas com algumas propriedades especificas sem usar explicitamente esta terminologia.

Definição 4.1. Sejam $X$ um espaço vetorial, $M$ um subconjunto de $X$ e $\lambda$ um cardinal.

- $M$ é dito $\lambda$-lineável se, e somente se $M \cup\{0\}$ contém um espaço vetorial com base de Hamel de dimensão $\lambda$.

- Se $X$ é um espaço vetorial topológico, $M$ é dito $\lambda$-espaçável se $M \cup\{0\}$ contém um subespaço fechado de $X$ de dimensão infinita e densidade $\lambda$.

$M$ é chamado de lineável (espaçável) se é $\omega$-lineável ( $\omega$-espaçável).

No caso que $X$ é um espaço de Banach e $M$ é o complementar de um subespaço fechado de $X$, o próximo teorema, tomado de [FKP13], prova que as duas definições anteriores são equivalentes. 
Teorema 4.2 (Teorema 3.1, [FKP13]). Seja $M$ um subespaço linear de um espaço vetorial $X$. As seguintes afirmações são equivalentes:

(a) $X \backslash M$ é lineável;

(b) O quociente $X / M$ é de dimensão infinita;

(c) Não existem $x_{1}, \ldots, x_{n} \in X$ tais que para todo $x \in X$ existem $c_{1}, \ldots, c_{n} \in \mathbb{R}$ e $y \in M$ tais que $x=c_{1} x_{1}+\cdots+c_{n} x_{n}+y$.

Adicionalmente, se $X$ é um espaço de Banach e $M$ é fechado, as afirmações acima são equivalentes $a$

(d) $X \backslash M$ é espaçável.

Esse teorema é usado em [FKP13] para demonstrar que o conjunto $\operatorname{NWM}(C(K))$ é espaçável sempre que $K$ não é fracamente Koszmider.

Teorema 4.3 (Teorema 3.4, [FKP13]). O conjunto dos operadores não multiplicadores fracos sobre $C(K)$ é vazio ou espaçável.

No caso $\operatorname{NWM}(C(K)) \neq \emptyset$, o espaço vetorial contido em $\operatorname{NWM}(C(K))$ construído na prova do Teorema 4.3 é o fecho do espaço gerado por enumeráveis elementos de $\mathrm{NWM}(C(K))$, portanto é Banach porque é um subespaço fechado de $\mathcal{L}(C(K))$, e tem densidade $\omega$. Como nenhum espaço de Banach tem dimensão de Hamel enumerável então pelo teorema anterior é possível concluir que $\operatorname{NWM}(C(K))$ é c-lineável e espaçável.

$\mathrm{Na}$ próxima seção provaremos que, se $C(K)$ tem $c_{0}$ como quociente, então existe um subespaço de $\mathcal{L}(C(K))$ com densidade $\mathfrak{c}$ e contido em $\operatorname{NWM}(C(K)) \cup\{0\}$, isto é, $\operatorname{NWM}(C(K))$ é $\mathfrak{c}$-espaçável.

\subsection{Espaçabilidade dos operadores não multiplicadores fracos de $C(K) \operatorname{com} c_{0}$ como quociente}

Pelo Teorema 1.8, a existência de operadores não fracamente compactos $T: C(K) \rightarrow X$, onde $X$ é um espaço de Banach, pode acontecer apenas nos casos que $X$ contém um subespaço isomorfo a $c_{0}$. A recíproca não é verdadeira, visto que, pelo Teorema 3.10, existem $K$ e $L$ compactos infinitos tais que todo operador de $C(K)$ em $C(L)$ é fracamente compacto, e sabe-se que $C(L)$, para $L$ infinito, contém uma cópia de $c_{0}$. Porém, pelo próximo resultado, de Jurado e Palacios, se existe uma transformação linear sobrejetora de $C(K)$ em $c_{0}$, essa não pode ser fracamente compacta.

Lema 4.4 (Corolário 2.2, [JP09]). Seja Y um espaço de Banach tal que existe um operador sobrejetivo compacto (respetivamente, fracamente compacto) de algum espaço normado a $Y$. Então $Y$ é de dimensão finita (respetivamente, reflexivo).

Como $c_{0}$ não é reflexivo, segue, desse resultado, que, se $C(K)$ tem $c_{0}$ como quociente, então existe um operador não fracamente compacto de $C(K)$ sobre $c_{0}$.

Teorema 4.5. Se $C(K)$ tem $c_{0}$ como quociente, então $N W M(C(K))$ é c-espaçável. 
Demonstração: Nosso objetivo será provar que, nas hipóteses do teorema, existe uma imersão linear de $l_{\infty}$ em $\mathcal{L}(C(K))$ com imagem contida em $N W M(C(K)) \cup\{0\}$. Isso será suficiente, visto que a densidade de $l_{\infty}$ é o peso de $\beta \mathbb{N}$, que é contínuo.

Supondo que $C(K)$ tem $c_{0}$ como quociente, fixe $T: C(K) \rightarrow c_{0}$ uma transformação linear, contínua e sobrejetora. Pelo Lema 4.4, $T$ não é fracamente compacta. Pelo Teorema 1.15, existem $\left(g_{n}\right)_{n \in \mathbb{N}}$ uma sequência limitada de funções contínuas, duas a duas disjuntas, e $\varepsilon>0$, tais que $\left\|T\left(g_{n}\right)\right\|>\varepsilon$, para todo $n \in \mathbb{N}$.

Escrevendo $f_{n}^{\prime}=g_{2 n+1}$ e $e_{n}=g_{2 n}$, as funções em cada uma das sequências $\left(f_{n}^{\prime}\right)_{n \geq 1}$ e $\left(e_{n}\right)_{n \geq 1}$ são duas a duas disjuntas e $\left\|T\left(f_{n}^{\prime}\right)\right\|>\varepsilon,\left\|T\left(e_{n}\right)\right\|>\varepsilon$ para todo $n \geq 1$.

Como $T\left(f_{n}^{\prime}\right) \in c_{0}$, existe uma sequência $\left(k_{n}\right)_{n \in \mathbb{N}}$ de números naturais tal que

$$
\left|T\left(f_{n}^{\prime}\right)\left(k_{n}\right)\right|>\varepsilon
$$

para todo $n \in \mathbb{N}$.

$\mathrm{O}$ conjunto $\left\{k_{n}: n \in \mathbb{N}\right\}$ é infinito. De fato, suponha, por absurdo, que tal conjunto é finito. Então existe $k \in \mathbb{N}$ tal que $k_{n}=k$ para infinitos $n$ 's e, portanto, existe uma subsequência $\left(f_{n_{l}}\right)_{l \in \mathbb{N}}$ tal que $\left|T\left(f_{n_{l}}\right)\left(k_{n_{l}}\right)\right|=\left|T\left(f_{n_{l}}\right)(k)\right|>\varepsilon$, para todo $l \in \mathbb{N}$. Note que para todo $r \in \mathbb{N}$ as funções $\sum_{l=1}^{r} f_{n_{l}}$ são limitadas por uma mesma constante $p$, porque $\left(f_{k_{l}}\right)_{l \in \mathbb{N}}$ é limitada de funções duas a duas disjuntas. Logo $\left\|T\left(\sum_{l=1}^{r} f_{n_{l}}\right)\right\| \geq\left|T\left(\sum_{l=1}^{r} f_{n_{l}}\right)(k)\right|>r \varepsilon$ contradizendo que $T$ é limitada.

Consequentemente, trabalhando com uma subsequência no lugar de $\left(f_{n}^{\prime}\right)_{n \in \mathbb{N}}$, podemos assumir que $k_{n}=k_{m}$ se, e somente se, $n=m$. Nestas condições definimos o conjunto $M$ por

$$
M:=\left\{k_{n}: n \in \mathbb{N}\right\}
$$

e, para cada $m \in M$, definimos $f_{m}=f_{j}^{\prime}$, onde $m=k_{j}$. Assim temos

$$
\left|T\left(f_{m}\right)(m)\right|=\left|T\left(f_{j}^{\prime}\right)\left(k_{j}\right)\right|>\varepsilon,
$$

para todo $m \in M$.

Podemos assumir, sem perda de generalidade, que $\left\|f_{n}\right\|=1$ e $e_{n}$ tem imagem contida em $[0,1]$, para todo $n \in \mathbb{N}$.

Tome uma partição $M=\bigcup_{n \in \mathbb{N}} A_{n}$, com cada $A_{n}$ infinito. Para cada $a=\left(a_{n}\right)_{n \in \mathbb{N}} \in l_{\infty}$, definimos a função $J^{a}: c_{0} \rightarrow C(K)$ dada por

$$
J^{a}(z)=\sum_{n \in \mathbb{N}} \sum_{m \in A_{n}} a_{n} z_{m} e_{m}
$$

onde $z=\left(z_{n}\right)_{n \in \mathbb{N}}$.

Afirmação 4.1. O operador $J^{a}$ está bem definido.

De fato, note que $J^{a}(z)=\sum_{n \in \mathbb{N}} b_{n} z_{n} e_{n}$, onde $b_{n}=a_{k}$, se $n \in A_{k}$, e $b_{n}=0$, se $n \in \mathbb{N} \backslash M$. Como $\left(a_{n}\right)_{n \in \mathbb{N}}$ é limitada, $\left(b_{n}\right)_{n \in \mathbb{N}}$ também o é. Logo, como $\left(z_{n}\right)_{n \in \mathbb{N}}$ converge a 0 , $\left(b_{n} z_{n}\right)_{n \in \mathbb{N}}$ também converge a 0 . Do fato de $\left(e_{n}\right)_{n \in \mathbb{N}}$ ser uma sequência limitada de funções duas a duas disjunta segue que $J^{a}(z)$ está bem definida e é limitada. Dado $x \in K$ provaremos que $J^{a}(z)$ é contínua em $x$. 
Se $e_{n}(x) \neq 0$, para algum $n \in \mathbb{N}$, então, pelo fato das funções serem disjuntas e contínuas, $J^{a}(z)$ é igual a $b_{n} z_{n} e_{n}$ numa vizinhança de $x$, o que garante a sua continuidade nesse ponto. Suponha, então, que $e_{n}(x)=0$, para todo $n \in \mathbb{N}$. Teremos $J^{a}(z)(x)=0$ e, para mostrarmos a continuidade de $J^{a}(z)$, é suficiente provarmos que, para todo $\varepsilon>0$, existe uma vizinhança aberta $V$ de $x$ tal que $\left|J^{a}(z)(y)\right|<\varepsilon$, para todo $y \in V$.

Fixe $\varepsilon>0$. Tome $n_{0}$ tal que, para todo $n>n_{0},\left|b_{n} z_{n}\right|<\varepsilon$. Tome $V=\bigcap_{n \leq n_{0}} V_{n}$, onde $V_{n}$ é uma vizinhança aberta de $x$ tal que $\left|b_{n} z_{n} e_{n}(y)\right|<\varepsilon$, para todo $y \in V_{n}$ (existe, pela continuidade de $\left.e_{n}\right)$. Teremos, pelo fato das funções $e_{n}$ 's serem duas a duas disjuntas e com imagens contidas em $[0,1],\left|J^{a}(z)(y)\right|<\varepsilon$, para todo $y \in V$.

Afirmação 4.2. $J^{a}$ é linear e contínuo.

A linearidade de $J^{a}$ pode ser trivialmente verificada. Do fato de termos $0 \leq e_{n} \leq 1$, para todo $n \in \mathbb{N}$, é imediato que $\left\|J^{a}(z)\right\| \leq\|a\| \cdot\|z\|$, tomando sempre a norma do supremo.

Agora, dado $a \in l_{\infty}$, definimos $T^{a}: C(K) \rightarrow C(K)$ por

$$
T^{a}=J^{a} \circ T .
$$

Afirmação 4.3. Se a $\neq 0$, então $T^{a}$ não é multiplicador fraco.

Para provar a afirmação, fixe $a \in l_{\infty} \backslash\{0\}$ e seja $k \in \mathbb{N}$ tal que $a(k) \neq 0$. Manteremos a notação usual de $a_{n}$ para $a(n)$.

Seja $\left(x_{n}\right)_{n \geq 1}$ em $K$ tal que $e_{n}\left(x_{n}\right)=1$. Note que $f_{n}\left(x_{n}\right)=0$, para todo $n \in \mathbb{N}$, pois, $e_{n}$ e $f_{n}$ são disjuntas. Fixando $m \in A_{k}$, e usando que $e_{j}\left(x_{m}\right)=0$, sempre que $j \neq m$, e $e_{m}\left(x_{m}\right)=1$, temos:

$$
T^{a}\left(f_{m}\right)\left(x_{m}\right)=\sum_{n \in \mathbb{N}} \sum_{j \in A_{n}} a_{n} T\left(f_{m}\right)(j) e_{j}\left(x_{m}\right)=a_{k} T\left(f_{m}\right)(m) .
$$

Portanto, $\left|T^{a}\left(f_{k}\right)\left(x_{k}\right)\right|>\left|a_{m}\right| \varepsilon$, provando que $T^{a}$ não é multiplicador fraco.

Afirmação 4.4. A aplicação $L: l_{\infty} \longrightarrow \mathcal{L}(C(K))$ dada por $L(a)=T^{a}$ é um isomorfismo sobre a imagem.

Da definição de $J^{a}$ e $T^{a}$ segue facilmente que $T^{a+\lambda b}=T^{a}+\lambda T^{b}$, para todos $a, b \in l_{\infty}$ e $\lambda \in \mathbb{R}$. Portanto, $L$ é linear. Como o operador nulo é multiplicador fraco, da afirmação 4.3 segue que $T^{a} \neq 0$, se $a \neq 0$, provando que $L$ é injetora.

O operador $L$ é contínuo porque $\left\|J^{a}\left(\left(\alpha_{n}\right)_{n \in \mathbb{N}}\right)\right\| \leq\|a\| \cdot\left\|\left(\alpha_{n}\right)_{n \in \mathbb{N}}\right\|$, logo

$$
\left\|T^{a}\right\| \leq \sup _{\|f\| \leq 1}\|a\|\|T\|\|f\| \leq\|a\| \cdot\|T\| .
$$

Agora vejamos que $L^{-1}$ é limitado. Verificamos, na prova da Afirmação 4.3, que $\left|T^{a}\left(f_{k}\right)\left(x_{k}\right)\right|>$ $\left|a_{m}\right| \varepsilon$, para todo $k \in A_{m}$. Como as $f_{k}$ 's tem norma 1 ,

$$
\sup _{\|f\| \leq 1}\left\|T^{a}(f)\right\| \geq \sup _{k \in \mathbb{N}}\left|T^{a}\left(f_{k}\right)\left(x_{k}\right)\right| \geq \sup _{m \in M}\left|a_{m}\right| \varepsilon=\|a\| \varepsilon
$$

ou seja, $\left\|T^{a}\right\| \geq\|a\| \varepsilon$ o que implica a continuidade de $L^{-1}$ e conclui a prova da Afirmação 4.4.

A afirmação 4.4 implica que a imagem de $L$ é um espaço Banach. 
Pela afirmação 4.3, o espaço $L\left(\ell_{\infty}\right)$ está contido em $N W M(C(K)) \cup\{0\}$, o que conclui a prova do teorema.

Corolário 4.6. Cada uma dessas condições implica que $N W M(C(K))$ é c-espaçável.

(a) K tem sequência convergente;

(b) $C(K)$ não é um espaço de Grothendieck.

Demonstração: Se $K$ tem sequência convergente não trivial, então pela Proposição $1.7, C(K)$ contém um subespaço complementado $E$ isometricamente isomorfo a $c_{0}$. Portanto, existe uma projeção $P$ de $C(K)$ sobre $E$, o que implica que $c_{0}$ é um quociente de $C(K)$. Logo, pelo Teorema 4.5, NWM $(C(K))$ é $\mathfrak{c}$-espaçável.

Agora, suponhamos que $C(K)$ não é um espaço de Grothendieck. Pela Proposição 5.3 de [Sch82], devida a Schachermayer, existe um subespaço complementado de $C(K)$ isometricamente isomorfo a $c_{0}$. Portanto, como na parte (a), o espaço $c_{0}$ é um quociente de $C(K)$ e o conjunto $N W M(C(K))$ é c-espaçável pelo Teorema 4.5 .

Em conjunto com R. Fajardo e L. Pellegrini, em [FGP19], foi demonstrado que se $K$ contém uma sequência convergente não trivial, então o conjunto $N W M(C(K))$ é c-lineável e o conjunto $N W M(C(K)) \cup\{0\}$ contém um espaço vetorial não separável. O Teorema 4.5 é uma melhora do resultado apresentado em [FGP19] porque obtemos um espaço vetorial contido em $N W M(C(K)) \cup$ $\{0\}$ que é fechado em $\mathcal{L}(C(K))$ e tem densidade $\mathfrak{c}$. Além disso, o Teorema 4.5 abrange uma classe maior de espaços $C(K)$ do que o resultado de [FGP19], pois $K$ ter sequência convergente não trivial implica que $C(K)$ tem $c_{0}$ como quociente, mas existem espaços $C(K)$ que contêm cópia complementada de $c_{0}$ e $K$ não tem sequências convergentes, por exemplo $K=\beta \mathbb{N} \times \beta \mathbb{N}$ não tem sequência convergente e $C(K)$ não é Grothendieck, para ver a prova deste fato indicamos [Cem84].

Outra observação é que, pelo Teorema 4.5 e o fato que se um espaço de Banach contém um subespaço isomorfo a $\ell_{\infty}$ esse subespaço é necessariamente complementado (ver Proposição 2.5.2 de [AK06]), concluímos que $\mathcal{L}(C(K))$ contém um subespaço complementado que todos seus elementos, exceto pelo zero, não são multiplicadores fracos. 


\section{Capítulo 5}

\section{Extensões compactos por sequências de funções duas a duas disjuntas}

Neste capítulo apresentamos a técnica da extensão por funções contínuas, usada para adicionar o supremo de uma sequência de funções contínuas de um compacto em [0,1], duas a duas disjuntas. Mostramos diversos lemas que serão úteis nas construções dos capítulos seguintes.

\subsection{Extensão forte e extensão completa}

Definição 5.1. Seja $K$ um espaço topológico compacto.

(i) Se $\left(f_{n}\right)_{n \in \mathbb{N}}$ uma sequência de funções contínuas de $K$ em $[0,1]$, duas a duas disjuntas, definimos

$$
\begin{aligned}
& D\left(\left(f_{n}\right)_{n \in \mathbb{N}}\right):=\bigcup\left\{U: U \text { é aberto e }\left\{n: \operatorname{supp}\left(f_{n}\right) \cap U \neq \emptyset\right\} \text { é finito }\right\} \\
& \text { e } \Delta\left(\left(f_{n}\right)_{n \in \mathbb{N}}\right):=K \backslash D\left(\left(f_{n}\right)_{n \in \mathbb{N}}\right) ;
\end{aligned}
$$

(ii) Se $K \subset[0,1]^{2^{\omega}}$ e $A \subset[0,1]$, denotamos por $K_{A}$ o conjunto $\{x \in K: x(0) \in A\}$.

Para frente, quando considerarmos o reticulado $C(K)$ sempre será com a ordem pontual usual.

Lema 5.2 (Lema 4.1, [Kos04]). Sejam K um espaço topológico compacto (Hausdorff) e $\left(f_{n}\right)_{n \in \mathbb{N}}$ uma sequência de funções contínuas de $K$ em $[0,1]$, duas a duas disjunta. Então:

(a) $f \in C(K)=\sup \left\{f_{n}: n \in \mathbb{N}\right\}$ no reticulado $C(K)$ se, e somente se,

$$
\left\{x \in K: \sum_{n \in \mathbb{N}} f_{n}(x) \neq f(x)\right\}
$$

é raro em $K$ (isto é, o seu fecho tem interior vazio);

(b) O conjunto $D\left(\left(f_{n}\right)_{n \in \mathbb{N}}\right)$ é aberto denso em $K$ e $\sum_{n \in \mathbb{N}} f_{n}$ está bem definida e é contínua em $D\left(\left(f_{n}\right)_{n \in \mathbb{N}}\right)$.

A partir de agora, quando usamos $\left.\left(\sum_{n \in \mathbb{N}} f_{n}\right)\right|_{D\left(\left(f_{n}\right)_{n \in \mathbb{N}}\right)}$ como função contínua, estamos usando implicitamente a parte $(b)$ do Lema 5.2 , mesmo sem fazer referência.

A definição de extensão de um espaço compacto por uma sequência de funções contínuas, duas a duas disjunta, foi introduzida por Koszmider em 2004. A definição de extensão completa foi apresentada posteriormente em 2012, por Fajardo. 
Definição 5.3 ([Kos04, Faj12]). Sejam $K$ um espaço compacto, $L \subset K \times[0,1]$, e $\left(f_{n}\right)_{n \in b}$ uma sequência de funções contínuas de $K$ em $[0,1]$, duas a duas disjuntas, para $b$ como sendo um subconjunto infinito de $\mathbb{N}$. Dizemos que $L$ é uma extensão de $K$ por $\left(f_{n}\right)_{n \in b}$ se $L$ é o fecho do gráfico da restrição $\left.\left(\sum_{n \in \mathbb{N}} f_{n}\right)\right|_{D\left(\left(f_{n}\right)_{n \in b}\right)}$. Além disso, sendo $L$ tal extensão, dizemos que $L$ é uma extensão forte de $K$ por $\left(f_{n}\right)_{n \in b}$ se o gráfico de $\sum_{n \in b} f_{n}$ está contido em $L$ e dizemos que que $L$ é uma extensão completa se, para todo $x \in K, \pi_{K, L}^{-1}[\{x\}]$ é unitário ou $\{x\} \times[0,1]$, onde $\pi_{K, L}=\left.\pi\right|_{L}$ e $\pi$ é a projeção canônica de $K \times[0,1]$ sobre $K$.

Se $b$ é um subconjunto infinito de $\mathbb{N}$ e $K$ um compacto, denotarmos por $K\left(\left(f_{n}\right)_{n \in b}\right)$ a extensão de $K$ pela sequência $\left(f_{n}\right)_{n \in b}$ de funções contínuas de $K$ em $[0,1]$. Usaremos a notação $K(b)$ para a mesma extensão quando estiver claro no contexto qual sequência de funções estamos considerando.

Como exemplo de uma extensão de um espaço compacto por funções contínuas que não é forte, considere o subespaço de $[0,1]^{2}$ dado por:

$$
K:=[0,1] \times\{0\} \cup\{0\} \times[0,1] \cup \bigcup_{n>1}([0,1] \times\{1 / n\})
$$

Note que $K$ é conexo e compacto. Usando o Lema de Urysohn encontramos funções $f_{n}: K \rightarrow$ $[0,1]$, duas a duas disjuntas, tais que $f_{n}([1 / 2] \times\{1 / n\})=\{1\}$ e $\left.\left.f_{n}(K \backslash(] 0,1] \times\{1 / n\}\right)\right)=\{0\}$. Assim, para $1 / 2 \leq x \leq 1$ acontece $\sum_{n>1} f_{n}(x, 0)=0$ e $\left.] 1 / 2,1\right] \times\{0\} \times\{0\}$ não pertence a $K\left(\left(f_{n}\right)_{n>1}\right)$.

Nos próximos exemplos mostramos que nem toda extensão completa é forte e nem toda extensão forte é completa. Para isso, começamos definindo $K_{0}:=[0,1]$ e $f_{n}: K_{0} \longrightarrow[0,1]$ dada por $f_{n}(x)=\left|\sin \left(\frac{\pi}{(1-x)}\right)\right|$, se $(n-1) / n \leq x \leq n /(n+1)$, e $f_{n}(x)=0$, caso contrário. Note que $\Delta\left(\left(f_{n}\right)_{n \in \mathbb{N}}\right)=\{1\}$ e $K_{0}\left(\left(f_{n}\right)_{n \in \mathbb{N}}\right)$ é o gráfico da restrição da função $x \mapsto\left|\sin \left(\frac{\pi}{(1-x)}\right)\right|$ ao intervalo $[0,1[$ unido com o segmento $\{1\} \times[0,1]$, ver gráfico 5.1

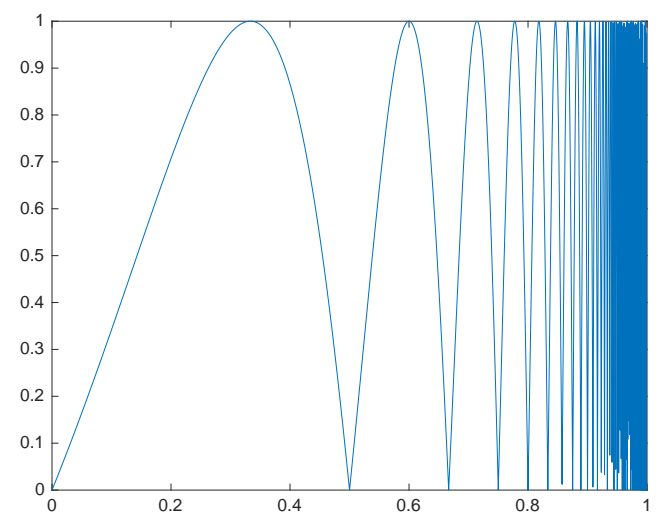

Figura 5.1: Extensão de $[0,1]$ por $\left(f_{n}\right)_{n \in \mathbb{N}}$

Exemplo 3. Sejam $K_{1}$ o compacto $K_{0}\left(\left(f_{n}\right)_{n \in \mathbb{N}}\right)$ e $g_{n}: K_{1} \rightarrow[0,1]$ a função definida por $g_{n}=f_{n} \circ \pi$ onde $\pi$ é a projeção sobre a primeira coordenada. Então $K_{1}\left(\left(g_{n}\right)_{n \in \mathbb{N}}\right)$ é uma extensão completa mas não forte.

Com efeito, note que $\Delta\left(\left(g_{n}\right)_{n \in \mathbb{N}}\right)=\{1\} \times[0,1]$ e, portanto, $g_{n}((1, y))=0$, para todos $y \in$ $[0,1]$ e $n \in \mathbb{N}$. Logo, $\{1\} \times[0,1] \times\{0\} \subset \operatorname{Gr}\left(\sum_{n \in \mathbb{N}} g_{n}\right)$. Por outro lado, note que $K_{1}\left(\left(g_{n}\right)_{n \in \mathbb{N}}\right)=$ $\left\{\left(x,\left|\sin \left(\frac{\pi}{(1-x)}\right)\right|,\left|\sin \left(\frac{\pi}{(1-x)}\right)\right|\right): 0 \leq x<1\right\} \cup\{(1, x, x)\}$ (veja gráfico 5.2) e, portanto, o gráfico de 
$\sum_{n \in \mathbb{N}} g_{n}$ não está contido em $K_{1}\left(\left(g_{n}\right)_{n \in \mathbb{N}}\right)$. Além disso, se $\pi_{K_{1}}$ denota a projeção de $K_{1}\left(\left(g_{n}\right)_{n \in \mathbb{N}}\right)$ sobre $K_{1}$, temos $\pi_{K_{1}}^{-1}\{(1, x)\}=\{(1, x, x)\}$.

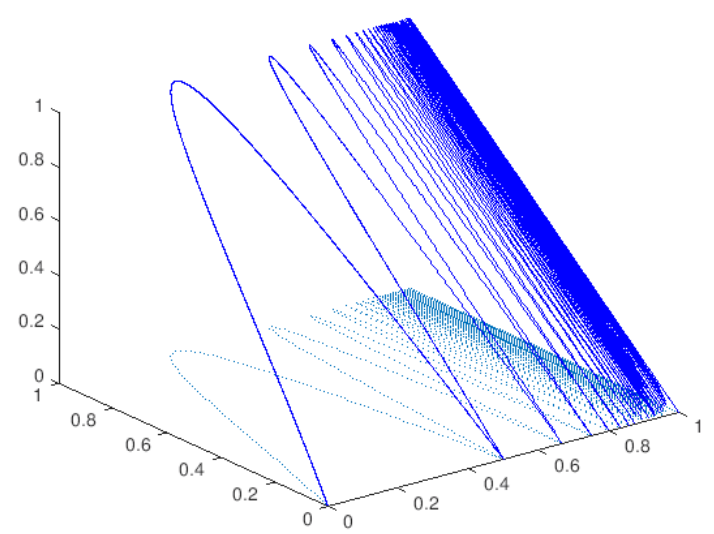

Figura 5.2: Extensão completa mas não forte

Exemplo 4. Sejam $K_{1}$ e $g_{n}$ para todo $n \in \mathbb{N}$ definidas como no exemplo anterior. Então a extensão de $K_{1}$ pela sequência de funções $\left(g_{n}\right)_{n \in 2 \mathbb{N}}$ é forte mas não completa.

Com efeito, note que $\Delta\left(g_{n}\right)_{n \in 2 \mathbb{N}}=\{1\} \times[0,1]$ e $\sum_{n \in 2 \mathbb{N}} g_{n}\left(x, f_{n}(x)\right)=0$ para todo $x \in$ $\left[\frac{2 n}{2 n+1}, \frac{2 n+1}{2 n+2}\right]$ (i.e nos intervalos correspondentes a $n$ impar), conforme a figura 5.3. Isso implica que $\{1\} \times[0,1] \times\{0\} \in K_{1}\left(\left(g_{n}\right)_{n \in 2 \mathbb{N}}\right)$, e portanto, a extensão $K_{1}\left(\left(g_{n}\right)_{n \in 2 \mathbb{N}}\right)$ é forte. Por outro lado, denotando por $\pi_{L, K_{1}}^{\prime}$ a projeção de $L:=K_{1}\left(\left(g_{n}\right)_{n \in 2 \mathbb{N}}\right)$ sobre $K_{1}$, temos que, para $y \in[0,1]$, $\pi_{L, K_{1}}^{-1}\{(1, y)\}=\{(1, y, 0),(1, y, y)\}$ e, portanto, a extensão de $K_{1}$ por $\left(\left(g_{n}\right)_{n \in 2 \mathbb{N}}\right)$ não é completa.

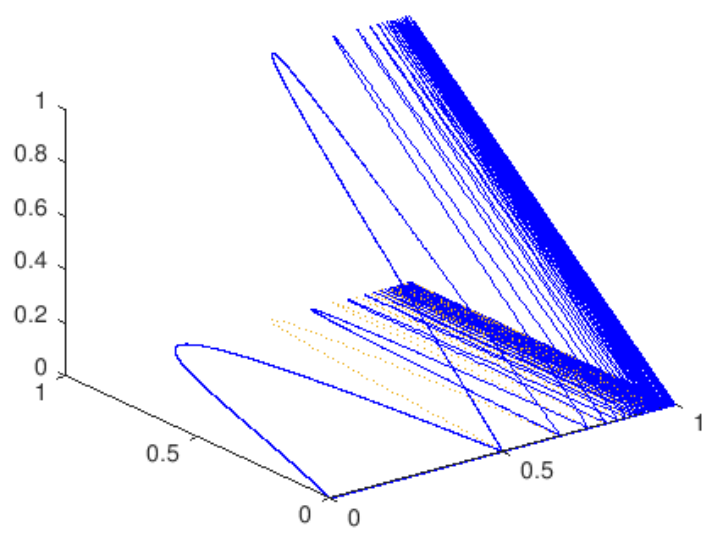

Figura 5.3: Extensão forte mas não completa

\subsection{Construções indutivas usando extensões por sequências de funções contínuas, duas a duas disjuntas.}

Um dos problemas de trabalhar com extensões por sequências de funções contínuas é a preservação da conexidade. Barbeiro e Fajardo em [BF17] apresentaram exemplos de extensões de 
espaços compactos por sequências de funções contínuas, duas a duas disjuntas, que não preservam conexidade.

Koszmider demonstrou que extensões fortes por funções contínuas preservam conexidade (ver Lema 4.4, [Kos04]). Como limite inverso preserva conexidade, se $K$ é obtido indutivamente a partir de $[0,1]^{2}$, usando extensões por funções nos ordinais sucessores e limites inversos nos ordinais limites, então $K$ é conexo. Mais ainda, pelo Lema 5.2, a existência de supremos de funções contínuas também é preservada, visto que extensões por sequências de funções contínuas preserva conjuntos raros. Tais fatos são resumidos no seguinte lema, provado por Koszmider:

Lema 5.4 (Lema 4.6, [Kos04]). Sejam $\beta$ um ordinal e $\left(K_{\alpha}\right)_{\alpha<\beta}$ tal que $K_{1}=[0,1]^{2}, K_{\alpha} \subset[0,1]^{\alpha}$ é compacto, $\pi_{\alpha}\left[K_{\alpha}\right]=K_{\alpha^{\prime}}$ para $\alpha^{\prime} \leq \alpha \leq \beta$, as extensões nos ordinais limites sendo limites inversos e as extensões de $K_{\alpha}$ para $K_{\alpha+1}$ sendo extensões fortes por funções contínuas de $K_{\alpha}$ em [0,1], duas a duas disjuntas. Então

(a) Se, para todos $n \in \mathbb{N}$ e $\alpha \leq \beta$, as funções $f, f_{n} \in C\left(K_{\alpha}\right)$ são tais que

$$
f=\sup \left\{f_{n}: n \in \mathbb{N}\right\},
$$

então

$$
f \circ \pi_{\alpha, \beta}=\sup \left\{f_{n} \circ \pi_{\alpha, \beta}: n \in \mathbb{N}\right\}
$$

(b) $K_{\beta} \backslash F$ é conexo, sempre que $F \subset K_{\beta}$ é finito.

O próximo lema garante que extensões completas também preservam conexidade.

Lema 5.5. Sejam $K$ e $L$ compactos e $\pi$ uma função contínua sobrejetora de $L$ sobre $K$. Se $K$ é conexo e $\pi^{-1}[\{x\}]$ é conexo, para todo $x \in K$, então $L$ é conexo.

Demonstração: Supondo $L$ desconexo, existem $U_{1}$ e $U_{2}$ abertos fechados disjuntos que formam uma separação de $L$. Em particular, $U_{1}$ e $U_{2}$ são compactos em $L$, porque $L$ é compacto e Hausdorff. Portanto, $\pi\left[U_{1}\right]$ e $\pi\left[U_{2}\right]$ são subconjuntos compactos de $K$. Como $K$ é compacto e Hausdorff, então $\pi\left[U_{1}\right]$ e $\pi\left[U_{2}\right]$ são fechados.

Note que $\pi\left[U_{1}\right] \cap \pi\left[U_{2}\right]=\emptyset$. Com efeito, se $x \in \pi\left[U_{1}\right] \cap \pi\left[U_{2}\right]$, então $\pi^{-1}[\{x\}] \cap U_{1} \neq \emptyset \mathrm{e} \pi^{-1}[\{x\}] \cap$ $U_{2} \neq \emptyset$, o que implica que $\pi^{-1}[\{x\}]$ é desconexo, porque $\pi^{-1}[\{x\}] \subset U_{1} \cup U_{2}$, contradizendo as hipóteses.

Finalmente, como $\pi$ é sobrejetora e $U_{1} \cap U_{2}=L$, temos que $\pi\left[U_{1}\right] \cup \pi\left[U_{2}\right]=K$, o que implica que $\pi\left[U_{1}\right]$ e $\pi\left[U_{2}\right]$ são uma separação de $K$, contradizendo que $K$ é conexo.

O próximo resultado é o análogo do Lema 5.4 adaptado para extensões completas, usando o lema 5.5.

Lema 5.6 (Lema 3.7, [Faj12]). Sejam $\beta$ um ordinal e $\left(K_{\alpha}\right)_{\alpha \leq \beta}$ uma sequência tal que $K_{2}=[0,1]^{2}$, $K_{\alpha} \subset[0,1]^{\alpha}$ é compacto, $K_{\alpha}$ é o limite do sistema inverso $\left(K_{\gamma}, \pi_{\gamma, \sigma}, \alpha\right)$, se $\alpha$ é um ordinal limite, e $K_{\alpha+1}$ é uma extensão completa de $K_{\alpha}$ por uma sequência de funções contínuas de $K_{\alpha}$ em [0,1], duas a duas disjuntas. Então: 
(i) Se $f, f_{n} \in C\left(K_{\alpha}\right)$, para $n \in \mathbb{N}$, e $\alpha<\beta$ tais que

$$
f=\sup \left\{f_{n}: n \in \mathbb{N}\right\}
$$

então

$$
f \circ \pi_{\alpha, \beta}=\sup \left\{f_{n} \circ \pi_{\alpha, \beta}: n \in \mathbb{N}\right\}
$$

(ii) Para todo conexo compacto $K^{\prime} \subset K_{2}, \pi_{2, \beta}^{-1}\left[K^{\prime}\right]$ é conexo. Em particular, $K_{\beta}$ é conexo.

As extensões de espaços compactos por sequências de funções contínuas duas a duas disjuntas preservam os conjuntos raros.

Lema 5.7 (Lema 4.3 (a), [Kos04]). Sejam K um espaço compacto, L uma extensão de $K$ por uma sequência $\left(f_{n}\right)_{n \in \mathbb{N}}$ de funções contínuas de $K$ em $[0,1]$, duas a duas disjuntas, e $\pi$ a projeção de $L$ sobre K. Se $M \subset K$ é raro em $K$ então $\pi^{-1}[M]$ é raro em $L$.

Aplicando indução e o Lema 5.7 é possível garantir que os conjuntos raros são preservados quando se fazem iteradas extensões por sequências de funções contínuas.

Lema 5.8. Sejam $\beta>2$ um ordinal e $\left(K_{\alpha}\right)_{\alpha \leq \beta}$ tal que $K_{2}=[0,1]^{2}, K_{\alpha} \subset[0,1]^{\alpha}$ é compacto e $\pi_{\alpha}\left[K_{\alpha}\right]=K_{\alpha^{\prime}}$, para $\alpha^{\prime} \leq \alpha \leq \beta, K_{\alpha}$ é o limite inverso de $\left(K_{\alpha^{\prime}}\right)_{\alpha^{\prime}<\alpha}$, se $\alpha \leq \beta$ é ordinal limite, e $K_{\alpha+1}$ é uma extensão de $K_{\alpha}$ por sequências de funções de $K_{\alpha}$ em $[0,1]$, contínuas, duas a duas disjuntas. Se $M$ é raro em $[0,1]^{2}$, então, para todo $2 \leq \alpha \leq \beta, \pi_{2, \alpha}^{-1}[M]$ é raro em $K_{\alpha}$.

Demonstração: A prova é por indução sobre $\alpha$. No passo sucessor, suponhamos que $\alpha<\beta$ e que $\pi_{2, \alpha}[M]$ é raro. Por 5.7, temos que $\pi_{\alpha, \alpha+1}^{-1}\left[\pi_{2, \alpha}^{-1}[M]\right]$ é raro e, portanto, $\pi_{2, \alpha+1}^{-1}[M]$ é raro.

Agora tomemos $\alpha$ ordinal limite e suponhamos que, para todo $2<\xi<\alpha, \pi_{2, \xi}^{-1}[M]$ é raro. Procedendo por absurdo, supomos que $U \subset K_{\alpha}$ é um aberto básico tal que $U \subset \overline{\pi_{2, \alpha}^{-1}[M]}$. Temos $U=\prod_{\xi<\alpha} V_{\xi} \cap K_{\alpha}$, com $V_{\xi} \neq[0,1]$ para apenas um número finito de $\xi$ 's. Para $\gamma>\max \left\{\xi: V_{\xi} \neq[0,1]\right\}$, $U_{\gamma}:=\prod_{\xi<\gamma} V_{\xi} \cap K_{\gamma}$ é aberto em $K_{\gamma}$ e tal que, se $x_{\gamma} \in U_{\gamma}$, então $\pi_{\gamma, \alpha}^{-1}\left[\left\{x_{\gamma}\right\}\right] \subset U$. Portanto, $U_{\gamma} \subset \pi_{\gamma, \alpha}[U] \subset \pi_{\gamma, \alpha}\left[\overline{\pi_{2}^{-1}[M]}\right]$. Vejamos que $U_{\gamma} \subset \overline{\pi_{2, \gamma}^{-1}[M]}$ contradizendo que $\pi_{2, \gamma}^{-1}[M]$ é raro.

Sejam $y_{\gamma} \in U_{\gamma}$ arbitrário e $W \subset U_{\gamma}$ um aberto contendo $y_{\gamma}$. Se $W \cap \pi_{2, \gamma}^{-1}[M]=\emptyset$. Temos $\pi_{\gamma, \alpha}^{-1}[W] \cap \pi_{\gamma, \alpha}^{-1}\left[\pi_{2, \gamma}^{-1}[M]\right]=\emptyset$. Como $\pi_{2, \alpha}^{-1}[M] \subset \pi_{\gamma, \alpha}^{-1}\left[\pi_{2, \gamma}^{-1}[M]\right]$, então $\pi_{\gamma, \alpha}^{-1}[W] \cap \pi_{2, \alpha}^{-1}[M]=\emptyset$, contradizendo que $\pi_{\gamma, \alpha}^{-1}\left[\left\{y_{\gamma}\right\}\right] \subset U \subset \overline{\pi_{2, \alpha}[M]}$. Assim, $U_{\gamma} \subset \overline{\pi_{2, \gamma}^{-1}[M]}$.

As extensões por sequências de funções contínuas, duas a duas disjuntas, não necessariamente preservam sequências convergentes. Ou seja, se $\left(x_{n}\right)_{n \in \mathbb{N}}$ é uma sequência de pontos distintos em um compacto $K$ que converge a $x$ e $\left(f_{n}\right)_{n} i n \mathbb{N}$ é uma sequência de funções de $K$ em [0,1], contínuas, duas a duas disjuntas, e $\left(x_{n}, t_{n}\right)_{n \in \mathbb{N}}$ é uma sequência em $K\left(\left(f_{n}\right)_{n \in \mathbb{N}}\right)$, não necessariamente essa sequência será convergente. De fato, por exemplo, usando normalidade de $K$, se pode considerar uma sequência de funções tal que $f_{n}\left(x_{2 n}\right)=1$ e $f_{n}\left(x_{m}\right)=0$ para $m \neq 2 n$ então $\left(x_{2 n}, 1\right) \rightarrow(x, 1)$ e $\left(x_{2 n+1}, 0\right) \rightarrow(x, 0)$ em $K\left(\left(f_{n}\right)_{n \in \mathbb{N}}\right)$.

No caso anterior, é possível fazer uma extensão de $K$ por uma subsequência de funções apropriada a modo de garantir que infinitas subsequências disjuntas de $\left(x_{n}\right)_{n \in \mathbb{N}}$ convirjam a $(x, 0)$. Esta ideia pode ser usada também para fazer uma extensão de um compacto $K$ que preserva que a 
intersecção dos fechos de uma família no máximo enumerável de subconjuntos enumeráveis de $K$ seja não vazia. Formalizamos esse argumento no seguinte lema, cuja prova é baseada na do Teorema 5.1 em [Faj12].

Lema 5.9. Sejam $K$ compacto metrizável, $x \in K, N \leq \omega$, e, para cada $m<N, Q_{L_{m}}:=\left\{q_{n m}: n \in\right.$ $\mathbb{N} \backslash 2 \mathbb{N}\}$ e $Q_{R_{m}}:=\left\{q_{n m}: n \in 2 \mathbb{N}\right\}$ subconjuntos enumeráveis disjuntos de $K$ tais que $\bar{Q}_{L_{m}} \cap \bar{Q}_{R_{m}}=$ $\{x\}$. Se $\left(f_{n}\right)_{n \in \mathbb{N}}$ é uma sequência de funções contínuas de $K$ em $[0,1]$ com suportes dois a dois disjuntos e tal que $\Delta\left(\left(f_{n}\right)_{n \in \mathbb{N}}\right)=\{x\}$, então existem um subconjunto infinito $b$ de $\mathbb{N}$ e, para cada $m<N$, conjuntos infinitos $c_{m} \subset \mathbb{N} \backslash 2 \mathbb{N}$ e $c_{m}^{\prime} \subset 2 \mathbb{N}$ tais que

$$
q_{m n} \notin \operatorname{supp}\left(f_{k}\right), \quad \forall n \in c_{m} \cup c_{m}^{\prime}, \forall k \in b .
$$

Consequentemente, para qualquer extensão de $K$ pelas funções $\left(f_{n}\right)_{n \in b^{\prime}}$ com $b^{\prime} \subset b$, e para todo $m \in \mathbb{N}$, tem-se $\overline{\left\{q_{n m}^{\prime}: n \in \mathbb{N} \backslash 2 \mathbb{N}\right\}} \cap \overline{\left\{q_{n m}^{\prime}: n \in 2 \mathbb{N}\right\}} \neq \emptyset$, onde $q_{n m}^{\prime}=\left(q_{n m}, \sum_{k \in b^{\prime}} f_{k}\left(q_{n m}\right)\right)$.

Demonstração: Seja $\{x\}=\Delta\left(\left(f_{n}\right)_{n \in \mathbb{N}}\right)$. Construímos, por indução, sequências $\left(U_{n}\right)_{n \in \mathbb{N}},\left(V_{n}\right)_{n \in \mathbb{N}}$ de abertos contendo $x$ com $U_{n+1} \subset V_{n} \subset U_{n}$ e sequências crescentes de inteiros $\left(k_{n}\right)_{n \in \mathbb{N}}$ e $\left(l_{n}\right)_{n \in \mathbb{N}}$ tais que

- $k_{n} \in 2 \mathbb{N}$ se e somente se $n \in 2 \mathbb{N}$;

- $q_{k_{n} m} \in U_{n} \backslash V_{n}$ para todo $n \geq m$;

- $\operatorname{supp}\left(f_{l_{n}}\right) \subset V_{n} \backslash U_{n+1}$.

Além disso, assumimos:

- $\left\{U_{n}: n \in \mathbb{N}\right\}$ forma um sistema fundamental de vizinhanças abertas de $x$.

Para garantirmos essa última propriedade, basta escolhermos $U_{n}$ com diâmetro menor a $1 / n$, quando o definirmos posteriormente.

Note que $\operatorname{supp}\left(f_{n}\right) \rightarrow x$ (i.e. quaisquer aberto contendo $x$ contém todos, exceto um número finito, $\left.\operatorname{dos} \operatorname{supp}\left(f_{n}\right)\right)$. De fato, caso contrário existiriam uma vizinhança aberta $V$ de $x$ e $c \subset \mathbb{N}$ infinito tais que, para todo $n \in c$, existe $y_{n} \in \operatorname{supp}\left(f_{n}\right) \backslash V$. Pela compacidade de $K$, o conjunto $\left\{y_{n}: n \in c\right\}$ tem pontos de acumulação, mas se $y^{\prime}$ é ponto de acumulação de $\left\{y_{n}: n \in c\right\}$, então $y^{\prime} \neq x$, contradizendo que $\Delta\left(\left(f_{n}\right)_{n \in \mathbb{N}}\right)$ unitário.

Escolhemos $U_{0}$ uma vizinhança aberta de $x$. Definidos $U_{n},\left(k_{j}\right)_{j<n}$ e $\left(l_{j}\right)_{j<n}$, tomamos $k_{n}$ inteiro tal que:

- $k_{n}>k_{j}$, para todo $j<n$;

- $q_{k_{n} m} \in U_{n}$, se $m \leq n$ e $m<N$;

- $k_{n} \in 2 \mathbb{N}$ se, e somente se, $n \in 2 \mathbb{N}$.

Tome $V_{n} \subset U_{n}$ uma vizinhança aberta de $x$ tal que $q_{k_{j} m} \notin V_{n}$, sempre que $m<N$ e $m \leq j \leq n$. Escolha $l_{n}$ inteiro tal que $l_{n}>l_{j}$, para todo $j<n$, e $\operatorname{supp}\left(f_{l_{n}}\right) \subset V_{n}$.

Usando o fato de que os suportes são disjuntos, podemos assumir que $x \notin \operatorname{supp}\left(f_{l_{n}}\right)$. Assim, podemos escolher $U_{n+1} \subset V_{n}$ vizinhança aberta de $x$ tal que $\operatorname{supp}\left(f_{l_{n}}\right) \cap U_{n+1}=\emptyset$. 
Note que, se $N<\omega$, então, para $n \geq N, k_{n}$ é escolhido de forma a garantir que os $N$ pontos $q_{k_{n} 1}, q_{k_{n} 2}, \ldots, q_{k_{n} N}$ pertencem a $U_{n}$ enquanto que, para $N=\omega$, o número de pontos continua aumentando conforme $n$.

Definimos $b:=\left\{l_{n}: n \in \omega\right\}, c_{m}:=\left\{k_{n}: n \geq m, n\right.$ impar $\}$ e $c_{m}^{\prime}:=\left\{k_{n}: n \geq m, n\right.$ par $\}$. Sejam $m<N, n \in \omega$ e $n \geq m$ então $q_{k_{n} m} \in U_{n} \backslash V_{n}$ além disso,

$$
\operatorname{supp}\left(f_{l_{j}}\right) \subset V_{j} \backslash U_{j+1} .
$$

Se $n \leq j$, temos $q_{k_{n} m} \notin V_{n}, \operatorname{supp}\left(f_{l_{j}}\right) \subset V_{j} \subset V_{n}$. Se $n>j$, temos $q_{k_{n} m} \in U_{n} \subset U_{j}$, $\operatorname{supp}\left(f_{l_{j}}\right) \cap U_{j+1}=\emptyset$ e $\operatorname{supp}\left(f_{l_{j}}\right) \cap U_{n}=\emptyset$.

Nos dois casos temos $q_{k_{n} m} \notin \operatorname{supp}\left(f_{l_{j}}\right)$. Portanto, $b, c_{m}$ e $c_{m}^{\prime}$ verificam (5.1), para todo $m<N$.

Note que (5.1) implica que, para cada $m \in \mathbb{N}$, se $n \in c_{m} \cup c_{m}^{\prime}$ então $\left(q_{n m}, 0\right) \in K\left(\left(f_{n}\right)_{n \in b^{\prime}}\right)$ para qualquer, $b^{\prime} \subset b$ infinito. Portanto, como $\left\{U_{n}: n \in \mathbb{N}\right\}$ forma um sistema fundamental de vizinhanças abertas de $x$, para todo $m<N$ acontece que $(x, 0) \in \overline{\left\{q_{n m}^{\prime}: n \in \mathbb{N} \backslash \mathbb{N}\right\}} \cap \overline{\left\{q_{n m}^{\prime}: n \in 2 \mathbb{N}\right\}} \subset$ $K\left(\left(f_{n}^{\prime}\right)_{n \in b^{\prime}}\right)$, onde $q_{n}^{\prime}=\left(q_{n}, 0\right)$ se $q_{n} \in \Delta\left(\left(f_{n}\right)_{n \in b^{\prime}}\right.$ e $q_{n}^{\prime}=\left(q_{n}, \sum_{n \in b^{\prime}} f_{n}\left(q_{n}\right)\right)$ no outro caso.

O próximo lema é uma generalização do anterior, enfraquecendo a hipótese de que esses pares de subconjuntos de $K$ tenham todos a mesma intersecção no único ponto de $\Delta\left(\left(f_{n}\right)_{n \in \mathbb{N}}\right)$.

Lema 5.10. Sejam $K$ compacto, $Q_{L_{m}}:=\left\{q_{n m}: n \in \mathbb{N} \backslash 2 \mathbb{N}\right\}$ e $Q_{R_{m}}:=\left\{q_{n m}: n \in 2 \mathbb{N}\right\}$ subconjuntos de $K$ enumeráveis disjuntos e com $\bar{Q}_{L_{m}} \cap \bar{Q}_{R_{m}} \neq \emptyset$, para todo $m \in \mathbb{N}$. Dada uma sequência de funções contínuas de suportes dois a dois disjuntos $f_{n}: K \rightarrow[0,1]$ tal que $\Delta\left(\left(f_{n}\right)_{n \in \mathbb{N}}\right)$ é unitário, então existe um subconjunto infinito $b$ de $\mathbb{N}$ tal que para todo $b^{\prime} \subset b$ acontece

$$
\overline{\left\{q_{n m}^{\prime}: n \in \mathbb{N} \backslash 2 \mathbb{N}\right\}} \cap \overline{\left\{q_{n m}^{\prime}: n \in 2 \mathbb{N}\right\}} \neq \emptyset,
$$

onde $q_{n m}^{\prime}=\left(q_{n m}, \sum_{k \in b^{\prime}} f_{k}\left(q_{n m}\right)\right)$ e o fecho é tomado em $K\left(\left(f_{n}\right)_{n \in b^{\prime}}\right)$.

Demonstração: Seja $J:=\left\{m \in \mathbb{N}:\left(\bar{Q}_{L_{m}} \cap \bar{Q}_{R_{m}}\right) \backslash \Delta\left(\left(f_{n}\right)_{n \in \mathbb{N}}\right) \neq \emptyset\right\}$ e, para cada $m \in J$, fixe $x_{m} \in\left(\bar{Q}_{L_{m}} \cap \bar{Q}_{R_{m}}\right) \backslash \Delta\left(\left(f_{n}\right)_{n \in \mathbb{N}}\right)$. Logo, para cada $m \in J$ existe uma vizinhança $V_{m}$ de $x_{m}$ que intersecta no máximo um número finito de suportes das funções $f_{n}$ 's. Logo, para qualquer subconjunto infinito $b^{\prime}$ de $\mathbb{N}, K\left(\left(f_{n}\right)_{n \in b^{\prime}}\right)$ é localmente o gráfico de uma função contínua e, portanto, para qualquer $b^{\prime} \subset \mathbb{N}$ e para todo $m \in J,\left(x_{m}, \sum_{n \in b^{\prime}} f_{n}\left(x_{m}\right)\right) \in \overline{\left\{q_{n m}^{\prime}: n \in \mathbb{N} \backslash 2 \mathbb{N}\right\}} \cap \overline{\left\{q_{n m}^{\prime}: n \in 2 \mathbb{N}\right\}}$, onde o fecho é tomado em $K\left(\left(f_{n}\right)_{n \in b^{\prime}}\right)$.

Agora, se $m \in \mathbb{N} \backslash J$, então $\overline{\left\{q_{n m}^{\prime}: n \in \mathbb{N} \backslash 2 \mathbb{N}\right\}} \cap \overline{\left\{q_{n m}^{\prime}: n \in 2 \mathbb{N}\right\}}=\Delta\left(\left(f_{n}\right)_{n \in \mathbb{N}}\right)$. Reenumeramos $\left\{\left(L_{m}, R_{m}\right): m \in \mathbb{N} \backslash J\right\}$ de modo que se possa considerar $\mathbb{N} \backslash J=\left\{m^{\prime}: m^{\prime}<N\right\}$ onde $N=\omega$, no caso de $\mathbb{N} \backslash J$ ser infinito, e $N=|\mathbb{N} \backslash J|+1$ no caso de $\mathbb{N} \backslash J$ ser finito.

Pelo Lema 5.9 existe um subconjunto infinito $b$ de $\mathbb{N}$ tal que para qualquer $b^{\prime} \subset b$ infinito e para todo $m^{\prime}<N$ (portanto, para todo $m \in \mathbb{N} \backslash J$ ) acontece (5.2). Como todos os $m \in J$ verificam a condição (5.2), para qualquer subconjunto infinito $b^{\prime}$ de $\mathbb{N}$ e, em particular, para todo subconjunto infinito $b^{\prime}$ do $b$, a condição (5.2) se verifica para todo $\left(L_{m}, R_{m}\right)$, com $m \in \mathbb{N}$ e todo $b^{\prime} \subset b$.

Se $\tilde{K}$ é um subespaço de $K$, das propriedades do fecho quando tomado na topologia do su- 
bespaço, segue o fato elementar

$$
\overline{\left\{x \in \tilde{K}:\left.f_{n}\right|_{\tilde{K}}(x)>0\right\}^{\tau} \tilde{K}}=\overline{\left\{x \in K: f_{n}(x)>0\right\} \cap \tilde{K}} \cap \tilde{K},
$$

onde o índice $\tau_{\tilde{K}}$ indica que o fecho é tomado na topologia do subespaço $\tilde{K}$ de $K$. Quando $\tilde{K}$ é fechado em $K$, temos $\overline{\left\{x \in \tilde{K}:\left.f_{n}\right|_{\tilde{K}}(x)>0\right\}}{ }^{\tau} \tilde{K}=\overline{\left\{x \in \tilde{K}: f_{n}(x)>0\right\}}$.

Lembrando que os abertos na definição de $D\left(\left(\left.f_{n}\right|_{\tilde{K}}\right)_{n \in b}\right)$ são os do subespaço $\tilde{K}$. Das duas últimas igualdades temos

$$
\Delta\left(\left(\left.f_{n}\right|_{\tilde{K}}\right)_{n \in b}\right) \subset \Delta\left(\left(f_{n}\right)_{n \in b}\right) \cap \tilde{K}
$$

Logo,

$$
\operatorname{Gr}\left(\left.\left(\sum_{n \in b} f_{n}\right)\right|_{D\left(\left(f_{n}\right)_{n \in b}\right)}\right) \cap(\tilde{K} \times[0,1]) \subset \operatorname{Gr}\left(\left.\left(\left.\sum_{n \in b} f_{n}\right|_{\tilde{K}}\right)\right|_{D\left(\left(\left.f_{n}\right|_{\tilde{K}}\right)_{n \in b}\right)}\right) .
$$

A igualdade em (5.4) não necessariamente acontece, por exemplo, suponha que os suportes das $f_{n}$ 's estão contidos em $K \backslash \tilde{K}$ e convergem a um ponto $y \in \tilde{K}$, então $y \notin \Delta\left(\left(\left.f_{n}\right|_{\tilde{K}}\right)_{n \in b}\right)$ e $y \in \Delta\left(\left(f_{n}\right)_{n \in b}\right), \operatorname{assim}(y, 0) \in \operatorname{Gr}\left(\left.\left(\left.\sum_{n \in b} f_{n}\right|_{\tilde{K}}\right)\right|_{D\left(\left(\left.f_{n}\right|_{\tilde{K}}\right)_{n \in b}\right)}\right) \operatorname{mas}(y, 0) \notin \operatorname{Gr}\left(\left.\left(\sum_{n \in b} f_{n}\right)\right|_{D\left(\left(f_{n}\right)_{n \in b}\right)}\right) \cap(\tilde{K} \times$ $[0,1])$. Obviamente, a inclusão em (5.4) continua valendo quando tomamos o fechos em $K \times[0,1]$, este fato e a relação entre o fecho tomado na topologia do subespaço $\tilde{K} \times[0,1]$ e no espaço $K \times[0,1]$ implicam

$$
\overline{\operatorname{Gr}\left(\left.\left(\sum_{n \in b} f_{n}\right)\right|_{D\left(\left(f_{n}\right)_{n \in b}\right)}\right) \cap(\tilde{K} \times[0,1])} \subset \overline{\operatorname{Gr}\left(\left.\left(\left.\sum_{n \in b} f_{n}\right|_{\tilde{K}}\right)\right|_{D\left(\left(\left.f_{n}\right|_{\tilde{K}}\right)_{n \in b}\right)}\right)} \cap(\tilde{K} \times[0,1])=\tilde{K}\left(\left(\left.f_{n}\right|_{\tilde{K}}\right)_{n \in b}\right)
$$

Por fim, note que se $K\left(\left(f_{n}\right)_{n \in b}\right)$ é uma extensão forte de $K$ por $\left(f_{n}\right)_{n \in b}$, então $\tilde{K}\left(\left(\left.f_{n}\right|_{\tilde{K}}\right)_{n \in b}\right) \subset$ $K\left(\left(f_{n}\right)_{n \in b}\right)$.

Lema 5.11. Sejam K compacto (Hausdorff), metrizável (equivalentemente, com base enumerável), $O_{1}$ e $O_{2}$ abertos não vazios em $K$ tais que $\bar{O}_{1} \cap \bar{O}_{2}=\emptyset, h: \bar{O}_{1} \rightarrow \bar{O}_{2}$ um homeomorfismo, $Q=$ $\left\{q_{n}: n \in \mathbb{N}\right\}$ um subconjunto denso enumerável em $K, \mathcal{P}$ um conjunto de pares $(L, R) \in[\mathbb{N}]^{\omega} \times[\mathbb{N}]^{\omega}$ satisfazendo $\overline{\left\{q_{n}: n \in L\right\}} \cap \overline{\left\{q_{n}: n \in R\right\}} \neq \emptyset$, e $\left(f_{n}\right)_{n \in N}$ uma sequência de funções contínuas, duas a duas disjuntas tal que $f_{n} \circ h=\left.f_{n}\right|_{\bar{O}_{2}}$, para todo $n \in N$. Se

(a) $\Delta\left(\left(f_{n}\right)_{n \in N}\right)=\{x, h(x)\}$ para algum $x \in \bar{O}_{1}$;

(b) existem abertos $O_{1}^{\prime} \supset \overline{O_{1}}$ e $O_{2}^{\prime} \supset \overline{O_{2}}$ com fechos disjuntos tais que $\operatorname{supp}\left(f_{n}\right) \cap \overline{O_{1}^{\prime}} \rightarrow x$ e $\operatorname{supp}\left(f_{n}\right) \cap \overline{O_{2}^{\prime}} \rightarrow h(x) ;$

então existe um subconjunto infinito $N$ de $\mathbb{N}$ tal que, para todos $(L, R) \in \mathcal{P}$ e $b \subset N$ infinito, acontece

$$
\overline{\left\{q_{n}^{\prime}: n \in L\right\}} \cap \overline{\left\{q_{n}^{\prime}: n \in R\right\}} \neq \emptyset,
$$

onde $q_{n}^{\prime}=\left(q_{n}, \sum_{k \in b} f_{k}\left(q_{n}\right)\right)$ e o fecho é tomado em $K\left(\left(f_{n}\right)_{n \in b}\right)$.

Demonstração: Para cada $(L, R) \in \mathcal{P}$ seja $Z_{L R}=\overline{\left\{q_{n}: n \in L\right\}} \cap \overline{\left\{q_{n}: n \in R\right\}}$, com o fecho sendo tomado em $K$. Definindo $J:=\left\{(L, R) \in \mathcal{P}: Z_{L R} \backslash\{x, h(x)\} \neq \emptyset\right\}$, raciocinando como na primeira parte da prova do Lema 5.10, concluímos que para qualquer $b \subset \mathbb{N}$ infinito todos os $(L, R) \in J$ verificam (5.5). 
Portanto, para provarmos (5.5) podemos assumir que $Z_{L R} \subset\{x, h(x)\}$, pois no outro caso já está provado.

Sejam $\mathcal{P}_{1}=\left\{(L, P) \in \mathcal{P}: x \in Z_{L R} \subset\{x, h(x)\}\right\}$ e $\mathcal{P}_{2}=\left\{(L, P) \in \mathcal{P}: h(x) \in Z_{L R} \subset\{x, h(x)\}\right\}$, assumindo, sem perda de generalidade, que $\mathcal{P}_{1} \neq \emptyset$.

Note que $\left(\left.f_{n}\right|_{\overline{O_{1}^{\prime}}}\right)_{n \in \mathbb{N}}$ é uma sequência de funções duas a duas disjuntas, e por (b) e o fato que $\operatorname{supp}\left(\left.f_{n}\right|_{\overline{O_{1}^{\prime}}}\right) \subset \operatorname{supp}\left(f_{n}\right) \cap \overline{O_{1}^{\prime}}$, acontece $\Delta\left(\left(\left.f_{n}\right|_{\overline{O_{1}^{\prime}}}\right)_{n \in b}\right)=\{x\}$. Para simplificar a notação vamos denotar por $K^{\prime}$ o subespaço $\overline{O_{1}^{\prime}}$.

Se $(L, R) \in \mathcal{P}_{1}$ então $\left\{q_{n}: n \in L\right\} \cap K^{\prime}$ e $\left\{q_{n}: n \in R\right\} \cap K^{\prime}$ são infinitos. Sejam $L^{\prime}=\{n \in L$ : $\left.q_{n} \in K^{\prime}\right\}$ e $R^{\prime}=\left\{n \in R: q_{n} \in K^{\prime}\right\}$. Assim $\overline{\left\{q_{n}: n \in L^{\prime}\right\}}{ }^{\tau^{\prime}} \cap{\overline{\left\{q_{n}: n \in R^{\prime}\right.}}^{\tau_{K^{\prime}}}=Z_{L R} \cap K^{\prime}=\{x\}$. Após enumerar o conjunto $\left\{\left(L^{\prime}, R^{\prime}\right):(L, R) \in \mathcal{P}_{1}\right\}$ aplicamos o Lema 5.9 a $K^{\prime},\left(\left.f_{n}\right|_{K^{\prime}}\right)_{n \in \mathbb{N}}$ e as renumerações de $\left\{q_{n}: n \in L^{\prime}\right\}$ com os ímpares e de $\left\{q_{n}: n \in R^{\prime}\right\}$ com os pares, para cada $(L, R) \in \mathcal{P}_{1}$. Consequentemente, existe $N_{1} \subset \mathbb{N}$ tal que, para todo $b \subset N_{1}$,

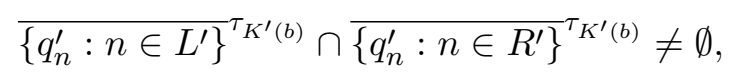

para todo $(L, R) \in \mathcal{P}_{1}$, e $q_{n}^{\prime}=q_{n} t_{n}, \operatorname{com} t_{n}=\left.\sum_{m \in b} f_{n}\right|_{K^{\prime}}\left(q_{m}\right)$.

Agora note que se $b \subset \mathbb{N}$ infinito, do fato que $\Delta\left(\left(\left.f_{n}\right|_{\tilde{K}}\right)_{n \in b}\right)=\{x\}=\Delta\left(\left(f_{n}\right)_{n \in b}\right) \cap \tilde{K}$ segue que $\operatorname{Gr}\left(\left.\left(\left.\sum_{n \in b} f_{n}\right|_{K^{\prime}}\right)\right|_{D\left(\left(\left.f_{n}\right|_{K^{\prime}}\right)_{n \in b}\right)}\right)=\operatorname{Gr}\left(\left.\left(\sum_{n \in b} f_{n}\right)\right|_{D\left(\left(f_{n}\right)_{n \in b}\right)}\right) \cap\left(K^{\prime} \times[0,1]\right)$ e, portanto, pelas propriedades do fecho quando tomado na topologia do subespaço,

$$
K^{\prime}\left(\left(\left.f_{n}\right|_{K^{\prime}}\right)_{n \in b}\right)=\overline{\operatorname{Gr}\left(\left.\left(\sum_{n \in b} f_{n}\right)\right|_{D\left(\left(f_{n}\right)_{n \in b}\right)}\right) \cap\left(K^{\prime} \times[0,1]\right)} .
$$

Enfatizamos que a topologia usada na primeira parte da igualdade é a do subespaço $K^{\prime} \times[0,1]$ enquanto que a da última é a do espaço $K \times[0,1]$.

Para cada $(L, R) \in \mathcal{P}_{1}$ acontece $q_{n}^{\prime} \in \operatorname{Gr}\left(\left.\left(\sum_{n \in b} f_{n}\right)\right|_{D\left(\left(f_{n}\right)_{n \in b}\right)}\right)$ para todos exceto possivelmente um par de $n$ 's em $L^{\prime} \cup R^{\prime}$. Por (5.7), para $b \subset \mathbb{N}, K^{\prime}\left(\left(\left.f_{n}\right|_{K^{\prime}}\right)_{n \in b}\right) \subset K\left(\left(f_{n}\right)_{n \in b}\right), \operatorname{logo}{\overline{\left\{q_{n}^{\prime}: n \in L^{\prime}\right\}}}^{\tau_{K^{\prime}(b)}}=$ $\overline{\left\{q_{n}^{\prime}: n \in L^{\prime}\right\}} \cap\left(K^{\prime} \times[0,1]\right) \subset \overline{\left\{q_{n}^{\prime}: n \in L\right\}}$. O mesmo acontece para $R$ e $R^{\prime}$ no lugar de $L$ e $L^{\prime}$, respectivamente. Logo, por (5.6), para cada $b \subset N_{1}$ e todo $(L, R) \in \mathcal{P}_{1}$ acontece $\overline{\left\{q_{n}^{\prime}: n \in L\right\}} \cap$ $\overline{\left\{q_{n}^{\prime}: n \in R\right\}} \neq \emptyset$.

Se $\mathcal{P}_{2} \backslash \mathcal{P}_{1}=\emptyset$, então tomando $N=N_{1}$ se verifica (5.5). Se $\mathcal{P}_{2} \backslash \mathcal{P}_{1} \neq \emptyset$, então para todo $(L, R) \in \mathcal{P}_{2} \backslash \mathcal{P}_{1}, Z_{L R}=\{h(x)\}$. Denotamos por $K^{\prime \prime}$ o subespaço $\overline{O_{2}^{\prime}}$ e aplicamos o Lema 5.9 a $K^{\prime \prime}$, $\left(\left.f_{n}\right|_{K^{\prime \prime}}\right)_{n \in N_{1}}$, e renumerações com os impares de $\left\{q_{n}: n \in L^{\prime \prime}\right\}$ e com os pares de $\left\{q_{n}: n \in R^{\prime \prime}\right\}$, onde $L^{\prime \prime}:=\left\{n \in L: q_{n} \in K^{\prime \prime}\right\}$ e $R^{\prime \prime}:=\left\{n \in R: q_{n} \in K^{\prime \prime}\right\}$, para $(L, R) \in \mathcal{P}_{2} \backslash \mathcal{P}_{1}$. Portanto, existe $N_{2} \subset N_{1}$ tal que para cada $b \subset N_{2}$

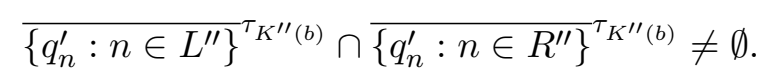

Continuando como antes para $\mathcal{P}_{1}$, por (5.7), se $b \subset N_{2}$, então para todo $(L, R) \in \mathcal{P}_{2} \backslash \mathcal{P}_{1}$ acontece $\overline{\left\{q_{n}^{\prime}: n \in L\right\}} \cap \overline{\left\{q_{n}: n \in R\right\}} \neq \emptyset$. Como $N_{2} \subset N_{1}$ então a interseç̧ão anterior é também não vazia para todo $L \in \mathcal{P}_{1}$ e, portanto, para todo $(L, R) \in \mathcal{P}$. Tomando $N=N_{2}$ completamos a prova do lema.

Se $K$ é um espaço compacto, metrizável e $U_{1}$ e $U_{2}$ são abertos disjuntos, com $\overline{U_{1}} \cap \overline{U_{2}}=\{x\}$ 
então existem uma sequência (infinita) de pontos em $U_{1}$ e uma sequência de pontos de $U_{2}$ ambas convergindo para $x$. Nosso objetivo agora será mostrar como se "elimina" um par de abertosborboleta bifurcando essa intersecção para pelo menos dois pontos: a saber, $(x, 0)$ e $(x, 1)$. Para isso, escolhemos funções que, em cada uma dessas sequências em $U_{1}$ e $U_{2}$, valem 0 em infinitos pontos da sequência e valem 1 (isto é, uma dessas funções vale 1) em infinitos pontos da sequência. Com isso garantiremos que, na extensão, para cada $i \in\{1,2\}$ existem duas sequências em $\pi^{-1}\left[U_{i}\right]$, sendo que uma converge para $(x, 0)$ e outra para $(x, 1)$, garantindo que $\pi^{-1}\left[U_{1}\right]$ e $\pi^{-1}\left[U_{2}\right]$ não formam um par de abertos-borboleta.

Extensões por funções contínuas que "eliminam" abertos-borboleta são usadas nas construções dos próximos capítulos. Ainda é preciso que a extensão preserve intersecções não vazias de fechos de pares de conjuntos enumeráveis, como no Lema 5.10 .

Lembramos que $[\mathbb{N}]^{\omega}$ denota o conjunto de todos os subconjuntos infinitos de $\mathbb{N}$.

Lema 5.12. Sejam $K \subset[0,1]^{\gamma}$ um espaço compacto sem pontos isolados, com $\gamma<\omega_{1}$, e $Q=\left\{q_{n}\right.$ : $n \in \mathbb{N}\}$ um conjunto denso em $K$. Defina $K_{1}=\overline{K_{\left[0, \frac{1}{3}[\right.}}$ e $K_{3}=\overline{\left.K_{]}, 1\right]}$.

Dados:

(a) $U$ e $V$ abertos disjuntos em $K$ e $z \in \bar{U} \cap \bar{V}$;

(b) um conjunto enumerável $\mathcal{P}$ de pares $(L, R) \in[\mathbb{N}]^{\omega} \times[\mathbb{N}]^{\omega}$ tais que $L \cap R=\emptyset$ e $\overline{\left\{q_{n}: n \in L\right\}} \cap$ $\overline{\left\{q_{n}: n \in R\right\}} \neq \emptyset$, em $K$;

(c) $h: K_{1} \rightarrow K_{3}$ uma função;

existem duas sequências $\left(U_{n}\right)_{n \in \mathbb{N}} e\left(V_{n}\right)_{n \in \mathbb{N}}$ de conjuntos abertos dois a dois disjuntos, uma sequência $\left(f_{n}\right)_{n \in \mathbb{N}}$ de funções contínuas, de $K$ em $[0,1]$, duas a duas disjuntas; sequências estritamente crescentes $\left(l_{n}^{i}\right)_{n \in \mathbb{N}}$ de naturais, para $i \in\{0,1\}$, e $a \subset \mathbb{N}$, infinito e co-infinito; tais que:

(d) $\left\{q_{l_{n}^{i}}: n \in \mathbb{N}\right\}$ é relativamente discreto e converge para $z$;

(e) $U_{n} \subset U$ e $V_{n} \subset V$ para todo $n \in \mathbb{N}$;

(f) toda vizinhança aberta de $z$ contém $U_{n}$ e $V_{n}$ para todos exceto um número finito de n's;

(g) $\operatorname{supp}\left(\left.f_{n}\right|_{K^{\prime}}\right) \subset U_{n} \cup V_{n}$, onde $K^{\prime}=K \backslash K_{1}$, caso $z \notin K_{1}$, e $K^{\prime}=K \backslash K_{3}$, caso $z \notin K_{3}$;

(h) $q_{l_{n}^{0}} \in U_{n}$ e $q_{l_{n}^{1}} \in V_{n}$;

(i) $f_{n}\left(q_{l_{n}^{i}}\right)=1$, para todos $n \in \mathbb{N}$ e $i \in\{0,1\}$;

(j) $K(b):=K\left(\left(f_{n}\right)_{n \in b}\right)$ é uma extensão completa, para qualquer $b \subset \mathbb{N}$ infinito;

(k) $(z, 1) \in \overline{\left\{q_{l_{n}^{1}}^{\prime}: n \in b\right\}} \cap \overline{\left\{q_{l_{n}^{0}}^{\prime}: n \in b\right\}}$, com o fecho tomado em $K(b)$, e $q_{m}^{\prime}:=\left(q_{m}, \sum_{n \in b} f_{n}\left(q_{m}\right)\right)$. Portanto, $(z, 1) \in \overline{\pi^{-1}[U]} \cap \overline{\pi^{-1}[V]}$. Além disso, se b é co-infinito, então $(z, 0) \in \overline{\left\{q_{l_{n}^{0}}^{\prime}: n \in \mathbb{N} \backslash b\right\}} \cap$ $\overline{\left\{q_{l_{n}^{1}}^{\prime}: n \in \mathbb{N} \backslash b\right\}}$ e, assim, $(z, i) \in \overline{\pi^{-1}[U]} \cap \overline{\pi^{-1}[V]}$ em $K(b)$, para cada $i \in\{0,1\}$;

(1) se $b \subset a$ é infinito, então para cada $(L, R) \in \mathcal{P}, \overline{\left\{q_{n}^{\prime}: n \in L\right\}} \cap \overline{\left\{q_{n}^{\prime}: n \in R\right\}} \neq \emptyset$, onde o fecho é tomado em $K(b)$ e $q_{m}^{\prime}$ é definido por $\left(q_{m}, \sum_{n \in b} f_{n}\left(q_{m}\right)\right)$;

(m) se hé um homeomorfismo, então $\left.f_{n}\right|_{K_{1}}=f_{n} \circ h$, para todo $n \in \mathbb{N}$. 
Demonstração: Usamos as ideias das provas do Lema 6.2 de [Kos04] e do Lema 5.1 de [Faj12].

Sejam $U$ e $V$ abertos como nas hipóteses do teorema, $z \in \bar{U} \cap \bar{V}$, e $\left\{r_{n}: n \in \mathbb{N}\right\}$ uma enumeração de $\mathbb{Q} \cap[0,1]$. Como $K$ é metrizável, existem sequências $\left(U_{n}\right)_{n \in \mathbb{N}}$ e $\left(V_{n}\right)_{n \in \mathbb{N}}$ de abertos disjuntos e sequências estritamente crescentes de naturais $\left(l_{n}^{i}\right)_{n \in \mathbb{N}}$, para $i \in\{0,1\}$, tais que:

(I) $U_{n} \subset U, V_{n} \subset V$ para todo $n \in \mathbb{N}$,

(II) para toda vizinhança aberta $O_{z}$ de $z$, temos $U_{n} \subset O_{z}$ e $V_{n} \subset O_{z}$ para todos exceto um conjunto finito de $n$ 's.

(III) $q_{l_{n}^{0}} \in U_{n}, q_{l_{n}^{1}} \in V_{n}$

Definimos $f_{n}: K \rightarrow[0,1]$ tais que:

(IV) $\operatorname{supp}\left(f_{n}\right) \cap K^{\prime} \subset U_{n} \cup V_{n}$, onde $K^{\prime}=K$, se $h$ não é homeomorfismo, e no caso contrário, $K^{\prime}=K \backslash K_{3}$, se $z \notin K_{3}$, e $K^{\prime}=K \backslash K_{1}$, se $z \notin K_{1}$;

(V) $f_{n}\left(q_{l_{n}^{0}}\right)=1=f_{n}\left(q_{l_{n}^{1}}\right)$;

(VI) $\left\{r_{0}, \ldots, r_{n}\right\} \subset \operatorname{Im}\left(\left.f_{n}\right|_{U_{n}}\right) \cap \operatorname{Im}\left(\left.f_{n}\right|_{V_{n}}\right)$;

(VII) se $h$ é homeomorfismo, $\left.f_{n}\right|_{K_{1}}=f_{n} \circ h$.

Provaremos que $\left(f_{n}\right)_{n \in \mathbb{N}}$ verifica o lema. Com efeito, note que (d) até (i) e (m) seguem diretamente da construção.

Antes de provar $(\mathrm{j})$ - (l), note que, se $x \in \bar{U}$, então $x \in \overline{U \cap K_{\left[0, \frac{1}{3}[\right.}} \cup \overline{U \cap\left(K \backslash K_{1}\right)}$. De fato, sejam $x \in \bar{U}=\overline{U \cap K_{1}} \cup \overline{U \cap\left(K \backslash K_{1}\right)}$ e $W_{x}$ um aberto contendo $x$. Se $x \in \overline{U \cap K_{1}}$, então $W_{x} \cap\left(U \cap K_{1}\right) \neq \emptyset, \operatorname{logo} W_{x} \cap U \neq \emptyset$ e $W_{x} \cap U \cap K_{[0,1 / 3[} \neq \emptyset$ porque $K_{1}=\overline{K_{\left[0, \frac{1}{3}\right.}[}$. Analogamente $x \in \overline{U \cap K_{] 2 / 3,1]}} \cup \overline{U \cap\left(K \backslash K_{3}\right)}$. Trocando $U$ por $V$ temos possibilidades análogas. Em consequência, se $z \in K \backslash\left(K_{1} \cup K_{3}\right)$ então $z \in \overline{U \cap\left(K \backslash\left(K_{1} \cup K_{3}\right)\right)} \cap \overline{V \cap\left(K \backslash\left(K_{1} \cup K_{3}\right)\right)}$. Logo podemos trocar, sem perda de generalidade, $U$ por $U \cap\left(K \backslash\left(K_{1} \cup K_{3}\right)\right)$ e $V$ por $V \cap\left(K \backslash\left(K_{1} \cup K_{3}\right)\right)$. Além disso, se $z \in K_{1}$, sem perda de generalidade podemos trocar $U$ por $U \cap\left(K \backslash K_{1}\right)$, no caso $z \in \overline{U \cap\left(K \backslash K_{1}\right)}$, e por $U \cap K_{[0,1 / 3[}$ no outro caso. Se $z \in K_{3}$ podemos trocar, sem perda de generalidade, $U$ por $U \cap\left(K \backslash\left(K_{1} \cup K_{3}\right)\right)$ se $z \in \overline{U \cap\left(K \backslash K_{3}\right)}$ e por $U \cap K_{] 2 / 3,1]}$ em outro caso. Resumindo, podemos supor, sem perda de generalidade que $U$ e $V$ verificam exatamente um das seguintes possibilidades:

P.1. $U, V \subset K \backslash\left(K_{1} \cup K_{3}\right)$;

P.2. $U \subset K_{\left[0, \frac{1}{3}[\right.}$ e $V \subset K \backslash\left(K_{1} \cup K_{3}\right)$;

P.3. $U \subset K \backslash\left(K_{1} \cup K_{3}\right)$ e $V \subset K_{\left.] \frac{2}{3}, 1\right]}$;

P.4. $U, V \subset K_{j}$ para algum $j \in\{1,3\}$.

Por outro lado, observe que para $b \subset \mathbb{N}$, as condições (I), (II) e (IV) e o fato que $K^{\prime}$ é aberto implicam $\Delta\left(\left(f_{n}\right)_{n \in b^{\prime}}\right) \cap K^{\prime}=\{z\}$. Em particular, se $h$ não é homeomorfismo então

$$
\Delta\left(\left(f_{n}\right)_{n \in b}\right)=\{z\}
$$


e, se $h$ é homeomorfismo, então, por (II), (IV) e (VII),

$$
\Delta\left(\left(f_{n}\right)_{n \in b}\right)=\{z\} \text { ou } \Delta\left(\left(f_{n}\right)_{n \in b}\right)=\{z, w\}
$$

onde $w=h(z)$ se $z \in K_{1}$ e $w=h^{-1}(z)$ se $z \in K_{3}$.

Vejamos que $(\mathrm{j})$ acontece para todo $b \subset \mathbb{N}$. Suponhamos, primeiro, que $\Delta\left(\left(f_{n}\right)_{n \in b}\right)=\{z\}$ e vamos provar que $\{z\} \times[0,1] \subset K\left(\left(f_{n}\right)_{n \in b}\right)$. Sejam $t \in[0,1]$ e $O \times I$ um aberto em $K\left(\left(f_{n}\right)_{n \in b}\right)$ contendo $(z, t)$ com $I \subset[0,1]$ intervalo aberto e $O$ aberto em $K$ contendo $z$.

Por (II), existe $N \in \mathbb{N}$ tal que para $n>N$, $\operatorname{supp}\left(f_{n}\right) \cap K^{\prime} \subset U_{n} \cup V_{n} \subset O$. Seja $n_{0} \in \mathbb{N}$ tal que $r_{n_{0}} \in I$. Se $b$ é um subconjunto infinito de naturais e $n \in b$ com $n>n_{0}$ por (III), $r_{n_{0}} \in \operatorname{Im}\left(\left.f_{n}\right|_{U_{n}}\right)$. Portanto, se $n>\max \left\{n_{0}, N\right\}$, existe $x \in \operatorname{supp}\left(f_{n}\right) \cap K^{\prime} \subset O$ tal que $f_{n}(x)=r_{0}$. $\operatorname{Assim}\left(x, r_{0}\right) \in O \times I$ e, como as $f_{n}$ 's tem suporte disjuntos, então $\left(x, r_{0}\right) \in \operatorname{Gr}\left(\left.\left(\sum_{n \in b} f_{n}\right)\right|_{D\left(\left(f_{n}\right)_{n \in b}\right)}\right.$ e, portanto, $(z, t) \in K(b)$.

No caso $\Delta\left(\left(f_{n}\right)_{n \in b}\right)=\{z, w\}$ os abertos $U$ e $V$ verificam alguma das possibilidades P.2. até P.4. A prova nos casos P.2 e P.3 é totalmente análoga pelo que provamos apenas para os casos P.2 e P.4. Neste último caso, ainda a prova para $j=1$ é a mesma que para $j=3$ pelo que podemos supor, sem perda de generalidade, que $U \subset K_{\left[0, \frac{1}{3}[\right.}$ e, portanto, $z \in K_{1}$.

Sejam $t \in[0,1], I$ um intervalo aberto em $[0,1]$ contendo $t, O^{\prime}$, e $O^{\prime \prime}$ abertos em $K$, com $z \in O^{\prime}$ e $w=h(z) \in O^{\prime \prime}$. Tomamos $\tilde{O} \subset O^{\prime \prime}$ aberto tal que $\tilde{O} \cap K_{3}=h\left[O^{\prime} \cap K_{1}\right] \cap O^{\prime \prime}$ e $O \subset O^{\prime}$ aberto tal que $O \cap K_{1}=h^{-1}\left[\tilde{O} \cap K_{3}\right]$. Seja $b \subset \mathbb{N}$ infinito, como $z \in O$, raciocinando como no caso $\Delta\left(\left(f_{n}\right)_{n \in b}\right)$ unitário, existem $n_{1}, n_{0} \in \mathbb{N}$ com $n_{1} \geq n_{0}$ tais que $r_{n_{0}} \in I$ e, para todo $n \in b$ com $n>n_{1}, r_{n_{0}} \in f_{n}[U]$ e $U_{n} \subset O$. Para cada $n \in b$ tal que $n>n_{1}$, seja $x_{n}$ tal que $\left.f_{n}\right|_{U}\left(x_{n}\right)=r_{n_{0}}$. Por (VII), $h\left(x_{n}\right) \in h\left[U_{n}\right] \subset \tilde{O} \cap K_{3}$ e $\left.f_{n}\right|_{h[U]}\left(h\left(x_{n}\right)\right)=r_{n_{0}}$. Como as $f_{n}$ 's são duas a duas disjuntas, então para $n \in b$ tal que $n>n_{1}, x_{n}, h\left(x_{n}\right) \notin \Delta\left(\left(f_{n}\right)_{n \in b}\right)$. Assim $\left(x_{n}, r_{n_{0}}\right),\left(h\left(x_{n}\right), r_{n_{0}}\right)$ pertencem ao gráfico da função $\left.\left(\sum_{n \in b} f_{n}\right)\right|_{D\left(\left(f_{n}\right)_{n \in b}\right)}$ e estão contidos em $O \times I$ e $\tilde{O} \times I$, respetivamente. Logo, $(z, t),(w, t) \in K(b)$ para $t \in[0,1]$ arbitrário. Note que realmente provamos que se $y \in \Delta\left(\left(f_{n}\right)_{n \in b}\right)$ então $\{y\} \times[0,1] \subset K(b)$ o que é mais do que $K(b)$ ser completa, isto implica, por exemplo, $K(b)$ também ser forte.

Para demonstrar $(\mathrm{k})$, note que, por $(\mathrm{V})$, para qualquer $b \subset \mathbb{N}$ infinito e todo $n \in b$ acontece $f_{n}\left(q_{l_{n}^{i}}\right)=1$, para $i \in\{0,1\}$. Além disso, para $b \subset \mathbb{N}$, (I)-(IV) implica que, para cada $i \in\{0,1\}$, $\left(q_{l_{n}^{i}}, 1\right) \stackrel{n \in b}{\longrightarrow}(z, 1)$. Como $U_{n} \cap U_{m}=\emptyset$ e $V_{n} \cap V_{m}=\emptyset$, sempre que $m \neq n$, e $U \cap V=\emptyset$, então, por (I)-(V), $\left(q_{l_{n}^{i}}, 1\right) \in \operatorname{Gr}\left(\left.\left(\sum_{n \in b} f_{n}\right)\right|_{D\left(\left(f_{n}\right)_{n \in b}\right)}\right)$, para todo $n \in b$ e $i \in\{0,1\}$, o que em conjunto com o anterior e (III) implica que para cada $b \subset \mathbb{N}$ infinito, $(z, 1) \in K\left(\left(f_{n}\right)_{n \in b}\right)$ e

$$
(z, 1) \in \overline{\left\{q_{l_{n}^{0}}^{\prime}: n \in b\right\}} \cap \overline{\left\{q_{l_{n}^{1}}^{\prime}: n \in b\right\}},
$$

onde $q_{l_{n}^{i}}^{\prime}=\left(q_{l_{n}^{i}}, 1\right)$ e o fecho é tomado em $K(b)$. Como $q_{l_{n}^{0}} \in U_{n} \subset U$, para todo $n \in \mathbb{N}$, então para $n \in b$ temos $\left(q_{l_{n}^{0}}, 1\right) \in \pi^{-1}[U] \subset K\left(\left(f_{n}\right)_{n \in b}\right)$. Analogamente, se $n \in b$, então $\left(q_{l_{n}^{1}}, 1\right) \in \pi^{-1}[V] \subset$ $K\left(\left(f_{n}\right)_{n \in b}\right)$. Logo, $(z, 1) \in \overline{\pi^{-1}[U]} \cap \overline{\pi^{-1}[V]} \subset K\left(\left(f_{n}\right)_{n \in b}\right)$, com o fecho como sendo tomado em $K(b)$.

Agora note que, se $\mathbb{N} \backslash b$ é infinito, então, por (IV), se $n \in b, f_{n}\left(q_{l_{m}^{i}}\right)=0$ para todo $m \in \mathbb{N} \backslash b$ e $i \in\{0,1\}$. Logo, por (II) e (III), $\left(q_{l_{n}^{i}}, 0\right) \rightarrow(z, 0)$, para $n \in \mathbb{N} \backslash b$, portanto

$$
(z, 0) \in \overline{\left\{\widetilde{q_{l_{n}^{0}}^{0}} 0: n \in \mathbb{N} \backslash b\right\}} \cap \overline{\left\{\widetilde{q_{l_{n}^{1}}} 0: n \in \mathbb{N} \backslash b\right\}}
$$


e, por $(\mathrm{I}),(z, 0) \in \overline{\pi^{-1}[U]} \cap \overline{\pi^{-1}[V]}$ em $K(b)$, sempre que $b$ é co-infinito; completando a prova de (k).

Agora vamos demonstrar (1). Suponhamos primeiro que $h$ não é homeomorfismo. Então para qualquer $b \subset \mathbb{N}$ infinito, $\left(f_{n}\right)_{n \in b}$ verifica (5.8). Renumerando para cada $(L, R) \in \mathcal{P}$ os conjuntos $\left\{q_{n}: n \in L\right\}$ com os ímpares e $\left\{q_{n}: n \in R\right\}$ com os pares e aplicando o Lema 5.10 achamos $a \subset \mathbb{N}$ tal que para todo $b \subset a$ acontece (l).

Agora supomos que $h$ é homeomorfismo. Dividimos em casos baseados em P.1. até P.4. Começamos supondo que $U$ e $V$ são como no caso P.1. (note que $z$ ainda pode pertencer a $K_{1}$ ou $K_{3}$ ). Temos que, para $b \subset \mathbb{N}, \Delta\left(\left(f_{n}\right)_{n \in b}\right)=\{z\}$. Aplicando o Lema 5.10 achamos $a \subset \mathbb{N}$ tal que para todo $b \subset a$ se verifica (l).

Agora suponhamos que $U$ e $V$ são como no caso P.2. ou no caso P.4. com $j=1$. Sem perda de generalidade podemos supor $U \subset K_{1}$ se for o caso P.2. Assim $z \in K_{1}$ e por (II) e (VII), $\Delta\left(\left(f_{n}\right)_{n \in \mathbb{N}}\right)=\{z, w\}$ com $w=h(z)$.

Aplicando o Lema 5.11 a $\left(f_{n}\right)_{n \in \mathbb{N}}, h: K_{1} \rightarrow K_{2}$, e $\mathcal{P}$ achamos $N \subset \mathbb{N}$ tal que (l) se verifica para todo $(L, R) \in \mathcal{P}$. Portanto, tomando $a=N$ provamos (l) nestes casos.

Por último, se $U$ e $V$ são como em P.3. ou em P.4. com $j=3$, então $z \in K_{3}$ e $\Delta\left(\left(f_{n}\right)_{n \in \mathbb{N}}\right)=$ $\{z, w\}$ com $w=h^{-1}(z)$, de novo aplicamos o Lema 5.11 para obter $N \subset \mathbb{N}$ tal que $\left(f_{n}\right)_{n \in \mathbb{N}}$ verifica (l), tomamos $a=N$. Isto completa a prova de (l).

No lema anterior a extensão é completa, enquanto nos Lemas 5.9 e 5.10 não necessariamente. O próximo lema permite fazer extensões completas de um compacto $K$ preservando algumas propriedades, mas ao contrário dos Lemas 5.9 e 5.10, as funções contínuas com que se faz a extensão não são exatamente uma subsequência das funções previamente dadas, mas são modificações dessas para garantir que a extensão seja completa.

No próximo lema, demonstramos que as funções $f_{n}^{\prime}$ podem ser escolhidas muito próximas das $f_{n}$ em relação à medida $\mu_{n}$ e podem ainda preservar um homeomorfismo entre abertos de $K$ previamente estabelecido.

Lema 5.13. Seja K compacto, metrizável e sem pontos isolados. Dados:

(a) uma sequência $\left(f_{n}\right)_{n \in \mathbb{N}}$ de funções contínuas de $K$ em $[0,1]$, duas a duas disjuntas;

(b) uma sequência $\left(\mu_{n}\right)_{n \in \mathbb{N}}$ de medidas de Radon sobre $K$;

(c) uma sequência $\left(\varepsilon_{n}\right)_{n \in \mathbb{N}}$ de números reais positivos;

(d) abertos $W_{0}$ e $W_{1}$ em $K$ tais que $\bar{W}_{0} \cap \bar{W}_{1}=\emptyset$;

(e) uma função $h: \bar{W}_{0} \longrightarrow \bar{W}_{1}$;

existe uma sequência $\left(f_{n}^{\prime}\right)_{n \in \mathbb{N}}$ de funções contínuas de $K$ em $[0,1]$, duas a duas disjuntas, tal que

(f) $\operatorname{supp}\left(f_{n}^{\prime}\right) \subset \operatorname{supp}\left(f_{n}\right)$;

(g) $\int\left(f_{n}-f_{n}^{\prime}\right) d\left|\mu_{n}\right|<\varepsilon_{n}$, para todo $n \in \mathbb{N}$;

(h) a extensão de $K$ por $\left(f_{n}^{\prime}\right)_{n \in b}$ é completa, para todo $b \subset \mathbb{N}$;

(i) se $h$ é um homeomorfismo e $\left.f_{n}\right|_{\bar{W}_{0}}=f_{n} \circ h$, para todo $n \in \mathbb{N}$, então $\left.f_{n}^{\prime}\right|_{\overline{W_{0}}}=f_{n}^{\prime} \circ h$, para todo $n \in \mathbb{N}$. 
Demonstração: Defina $V_{n}=\left\{x \in K: f_{n}(x)>0\right\}$ e fixe $\left(U_{n}\right)_{n \in \mathbb{N}}$ uma base enumerável de $K$ e $\left(r_{n}\right)_{n \in \mathbb{N}}$ uma enumeração de $\mathbb{Q} \cap[0,1]$. Defina $X_{i}=W_{i}$, se $i \in\{0,1\}$, e $X_{2}=K \backslash\left(\bar{W}_{0} \cup \bar{W}_{1}\right)$. Claramente, $\bigcup_{i \in 3} X_{i}$ é um aberto denso em $K$.

Para cada $n \in \mathbb{N}$ defina $I_{n}=\left\{i \leq n: U_{i} \cap V_{n} \cap\left(X_{0} \cup X_{2}\right) \neq \emptyset\right\}$ e fixamos uma sequência finita $\left(V_{i, j}^{n}\right)_{i \in I_{n}, j \leq n}$ de abertos não-vazios e duas a duas disjuntos, tal que, para cada $i \in I_{n}$ e $j \leq n$, $V_{i, j}^{n} \subset U_{i} \cap V_{n} \cap X_{0}$, se $U_{i} \cap V_{n} \cap X_{0} \neq \emptyset$, e $V_{i, j}^{n} \subset U_{i} \cap V_{n} \cap X_{2}$, caso contrário.

Assumimos, ainda, usando regularidade de $\mu_{n}$ e o fato do espaço não conter pontos isolados, que $\left|\mu_{n}\right|\left(V_{i, j}^{n}\right)<\frac{\varepsilon_{n}}{4 n^{2}}$ e, caso $V_{i, j}^{n} \subset X_{0},\left|\mu_{n}\right|\left(h\left[V_{i, j}^{n}\right]\right)<\frac{\varepsilon_{n}}{4 n^{2}}$. Note que isso já garantirá a propriedade $(g)$ do enunciado, visto que definiremos a função $f_{n}^{\prime}$ alterando $f_{n}$ apenas nos abertos $V_{i, j}^{n}$ e $h\left[V_{i, j}^{n}\right]$.

Usando normalidade de $K$, para cada $V_{i, j}^{n}$ fixe um aberto não vazio $W_{i, j}^{n}$ tal que $\overline{W_{i, j}^{n}} \subset V_{i, j}^{n}$ e, usando o Lema de Urysohn, uma função $g_{i, j}^{n}: V_{i, j}^{n} \longrightarrow[0,1]$ tal que $r_{j} \in \operatorname{Im}\left(g_{i, j}^{n}\right)$ e $g_{i, j}^{n}(x)=f_{n}(x)$, se $x \in V_{i, j}^{n} \backslash \overline{W_{i, j}^{n}}$

Defina $f_{n}^{\prime}$ como:

- $f_{n}^{\prime}(x)=g_{i, j}^{n}(x)$, se $x \in V_{i, j}^{n}$, para algum $i \in I_{n}$ e $j \leq n$;

- $f_{n}^{\prime}(x)=g_{i, j}^{n}\left(h^{-1}(x)\right)$, se $x \in h\left[V_{i, j}^{n}\right]$, para algum $i \in I_{n}$ e $j \leq n$;

- $f_{n}^{\prime}(x)=f_{n}(x)$, nos outros casos.

Note que cada $f_{n}^{\prime}$ é contínua e satisfaz as condições $(f),(g)$ e $(i)$ do lema. Falta provar a condição $(h)$. Para isso, sejam $b \subset \mathbb{N}$ infinito, $x \in \Delta\left(\left(f_{n}^{\prime}\right)_{n \in b}\right), r \in \mathbb{Q} \cap[0,1]$ e $U$ aberto tal que $x \in U$. Precisamos provar que existem $n \in b$ e $y \in U$ tais que $f_{n}^{\prime}(y)=r$.

Caso 1: $x \in \overline{X_{0} \cup X_{2}}$.

Tome $j$ tal que $r_{j}=j$ e $i$ tal que $U_{i} \subset U$ e $x \in U_{i}$. Temos que $i \in I_{n}$, para infinitos $n$ 's em $b$ tais que $n \geq i$. Agora fixe $n \geq i, j$ tal que $n \in b$. Temos que $V_{i, j}^{n} \subset U_{i} \cap V_{n} \cap\left(X_{1} \cup X_{2}\right)$ e existe $y \in V_{i, j}^{n}$ tal que $f_{n}^{\prime}(y)=g_{i, j}^{n}(y)=r_{j}=r$, como queríamos.

Caso 2: $\quad x \notin \overline{X_{0} \cup X_{2}}$.

Nesse caso temos $x \in \bar{W}_{1}$. Seja $x^{\prime}=h^{-1}(x)$. Pela continuidade de $h$, existe $i \in \mathbb{N}$ tal que $x^{\prime} \in U_{i}$ e $h\left[U_{i} \cap \overline{W_{0}}\right] \subset U$. Repetindo o argumento do Caso 1, concluímos que existe $y \in U_{i} \cap V_{n} \cap W_{0}$ tal que $f_{n}^{\prime}(y)=r$ e, por construção, $f_{n}^{\prime}(h(y))=r$.

Nas extensões de compactos por uma sequência $\left(f_{n}\right)_{n \in \mathbb{N}}$ de funções duas a duas disjuntas, a dificuldade para preservar propriedades de $K$ emerge dos pontos que pertencem $\Delta\left(\left(f_{n}\right)_{n \in \mathbb{N}}\right)$. Se este conjunto for vazio, a extensão de $K$ é justamente o gráfico de uma função contínua e todas as propriedades topológicas de $K$ seriam transferidas para $K\left(\left(f_{n}\right)_{n \in \mathbb{N}}\right)$. No próximo lema provamos que é possível obter um subconjunto $b$ de $\mathbb{N}$ e funções $f_{n}^{\prime}$ a partir das $f_{n}$ 's tais que o conjunto $\Delta\left(\left(f_{n}^{\prime}\right)_{n \in b}\right)$ é muito pequeno ou não intersecta um conjunto enumerável de pontos em $K$ previamente fixado.

Lema 5.14. Sejam $1 / 3<r<2 / 3, \varepsilon>0, K \subset[0,1]^{\gamma}$ um espaço metrizável, compacto e sem pontos isolados, com $\gamma<\omega_{1}, Q=\left\{q_{n}: n \in \mathbb{N}\right\}$ um conjunto denso em $K$, e $\left(x_{n}\right)_{n \in \mathbb{N}}$ uma sequência infinita em $K$. Seja $\left(\mu_{n}\right)_{n \in \mathbb{N}}$ uma sequência de medidas de Radon tais que $\left|\mu_{n}\right|\left(K \backslash \overline{K_{[0, r}}\right)=0$, para todo 
$n \in \mathbb{N}$, ou $\left|\mu_{n}\right|\left(K \backslash \overline{K_{] r, 1]}}\right)=0$, para todo $n \in \mathbb{N}$. Suponha que $h: K_{1} \rightarrow K_{3}$, onde $K_{1}:=\overline{K_{\left[0, \frac{1}{3}[\right.}}$ e $K_{3}:=\overline{\left.K_{1}, 1\right]}$, definida por $h(x)(0)=1-x(0)$ e $h(x)(\alpha)=x(\alpha)$, para $\alpha>0$ é uma função contínua e bijetora (e, portanto, homeomorfismo). Se $\left(f_{n}\right)_{n \in \mathbb{N}}$ é uma sequência de funções contínuas de $K$ em $[0,1]$, duas a duas disjuntas, tal que $f_{n}(x)=f_{n}(h(x))$, para todo $x \in K_{1}$, e $\left|\int f_{n} d \mu_{n}\right|>\varepsilon$, para todo $n \in \mathbb{N}$, então existem $N_{2} \subset \mathbb{N}$ infinito, $\delta>0$ e funções contínuas $f_{n}^{\prime} \operatorname{com} \operatorname{supp}\left(f_{n}^{\prime}\right) \subset \operatorname{supp}\left(f_{n}\right)$ tais que:

(a) $\left|\int f_{n}^{\prime} d \mu_{n}\right|>\delta$ e $\Sigma\left\{\int f_{m}^{\prime} d\left|\mu_{n}\right|: m \neq n, m \in N_{2}\right\}<\delta / 3$, para todo $n \in N_{2}$;

(b) $L=K\left(\left(f_{n}^{\prime}\right)_{n \in b}\right)$ é uma extensão completa, para todo $b \subset N_{2}$;

(c) $\left.f_{n}^{\prime}\right|_{K_{1}}=f_{n}^{\prime} \circ h$;

(d) para $\Delta\left(\left(f_{n}^{\prime}\right)_{n \in N_{2}}\right)$ acontece exatamente uma das seguintes possibilidades:

(I) $x_{m} \notin \Delta\left(\left(f_{n}^{\prime}\right)_{n \in N_{2}}\right)$, para todo $m \in \mathbb{N}, h\left(x_{m}\right) \notin \Delta\left(\left(f_{n}^{\prime}\right)_{n \in N_{2}}\right)$, sempre que $x_{m} \in K_{1}$, e $h^{-1}\left(x_{m}\right) \notin \Delta\left(\left(f_{n}^{\prime}\right)_{n \in N_{2}}\right)$, sempre que $x_{m} \in K_{3}$;

(II) $\Delta\left(\left(f_{n}^{\prime}\right)_{n \in N_{2}}\right)$ é unitário, $\left.f_{n}^{\prime}\right|_{K_{1} \cup K 3} \equiv 0$, para todo $n \in N_{2}$, e $\Delta\left(\left(f_{n}^{\prime}\right)_{n \in N_{2}}\right) \subset\left(K \backslash\left(K_{1} \cup\right.\right.$ $\left.\left.K_{3}\right)\right) \cup\left(K_{i} \cap K_{\left[\frac{1}{3}, \frac{2}{3}\right]}\right)$, onde $i=1$ se $\left|\mu_{n}\right|\left(K \backslash \overline{K_{[0, r}}\right)=0$ e $i=3$ se $\left|\mu_{n}\right|\left(K \backslash \overline{K_{] r, 1]}}\right)=0$, para todo $n \in \mathbb{N}$;

(III) $\Delta\left(\left(f_{n}^{\prime}\right)_{n \in N_{2}}\right)=\{x, h(x)\}$, para algum $x \in K_{1}$, e $\operatorname{supp}\left(f_{n}^{\prime}\right) \cap K_{[0, r]} \rightarrow x$ e $\operatorname{supp}\left(f_{n}^{\prime}\right) \cap$ $K_{[s, 1]} \rightarrow h(x)$, para algum $r<s<2 / 3$.

Demonstração: A prova segue a do Lema 3.10 de [Faj12], com a diferença de que precisamos preservar o homeomorfismo $h$. Para cada $n \in \mathbb{N}$ denotamos por $A_{n}$ o aberto $\left\{x \in K: f_{n}(x)>0\right\}$ e por $\Delta\left(\left(A_{n}\right)_{n \in \mathbb{N}}\right)$ o conjunto $\left\{x \in K\right.$ : toda vizinhança de $x$ intersecta infinitos $A_{n}$ 's $\}$. Assumimos, sem perda de generalidade, que $\left|\mu_{n}\right|\left(K \backslash \overline{\left.K_{[0, r[}\right)}\right)=0$, sendo a prova do outro caso análoga.

Para demonstrar o lema dividimos a prova em dois casos:

CASO 1. Existem $\delta^{\prime}>0$ e $x \in K$ tal que para toda vizinhança aberta $V$ de $x$ e para todo $m \in \mathbb{N}$, existe $k>m$ tal que $\left|\mu_{k}\right|\left(A_{k} \cap V\right)>\delta^{\prime}$

Neste caso, $x \notin A_{n}$, para todo $n \in \mathbb{N}$, porque as $f_{n}$ 's são duas a duas disjuntas. Além disso, pela hipótese sobre a as medidas, necessariamente temos $x \in \overline{K_{[0, r}}$.

Como $K$ é metrizável, podemos tomar uma sequência $\left(V_{n}\right)_{n \in \mathbb{N}}$ decrescente que é um sistema fundamental de vizinhanças abertas de $x, \operatorname{com} V_{n} \subset B_{\frac{1}{n}}(x)$ e $V_{n} \subset K_{[0, r+\sigma[}$, para $0<\sigma<\left(\frac{2}{3}-r\right) / 2$ fixado.

Supondo primeiro que $x \notin K_{1} \cap K_{\left\{\frac{1}{3}\right\}}$, construímos sequências crescentes de inteiros positivos $k_{n}$ e $j_{n}$ tais que $\left|\mu_{j_{n}}\right|\left(A_{j_{n}} \cap V_{k_{n}}\right)>\delta^{\prime}$ e $V_{k_{1}} \subset K_{1}$ se $x(0)<\frac{1}{3}$ e $V_{k_{1}} \subset K_{[0, r+\sigma[} \backslash K_{1}$ no caso contrário.

Definimos $A_{j_{n}}^{\prime}:=A_{j_{n}} \cap V_{k_{n}}$ e fixamos $\delta_{j_{n}}>\delta^{\prime}$ tal que $\left|\mu_{j_{n}}\left(A_{j_{n}}^{\prime}\right)\right|>\delta_{j_{n}}$. Note que a condição sobre as medidas, neste caso, implica que $A_{j_{n}} \cap K_{[0, r[} \neq \emptyset$ e $\left|\mu_{j_{n}}\right|\left(A_{j_{n}} \cap \overline{K_{[0, r[}}\right)=\left|\mu_{j_{n}}\right|\left(A_{j_{n}}\right)$. Logo, usando normalidade de $K$ e regularidade das medidas, achamos $B_{j_{n}} \subset A_{j_{n}}^{\prime} \cap \overline{K_{[0, r[}}$ fechado tal que $\left|\mu_{j_{n}}\left(B_{j_{n}}\right)\right|>\delta_{j_{n}}$ e $\left|\mu_{j_{n}}\right|\left(A_{j_{n}}^{\prime} \backslash B_{j_{n}}\right)<\delta^{\prime}-\delta_{j_{n}}$.

Se $x(0)<\frac{1}{3}$, então $A_{j_{n}}^{\prime} \subset K_{1}$ e, portanto, usando o Lema de Urysohn, podemos tomar $f_{j_{n}}^{\prime}$ : $K \rightarrow[0,1]$ tal que

$$
\left.f_{j_{n}}^{\prime}\right|_{B_{j_{n}}} \equiv 1,\left.\quad f_{j_{n}}^{\prime}\right|_{\left(K \backslash A_{j_{n}}^{\prime}\right) \backslash K_{3}} \equiv 0,\left.\quad f_{j_{n}}^{\prime}\right|_{K_{3}}=f_{j_{n}}^{\prime} \circ h^{-1}
$$


, que é contínua porque $V_{k_{1}} \subset K_{1}$. Assim, tomando $N_{1}=\left\{j_{n}: n \in \mathbb{N}\right\}, \Delta\left(\left(f_{n}^{\prime}\right)_{n \in N_{1}}\right)=\{x, h(x)\}$ porque $A_{n}^{\prime} \subset V_{n}, h\left(A_{n}^{\prime}\right) \subset h\left(V_{n}\right)$, para todo $n \in N_{1},\left(V_{n}\right)_{n \in N_{1}}$ converge a $x$ e $\left(h\left(V_{n}\right)\right)_{n \in N_{1}}$ converge a $h(x)$. É imediato da definição das $f_{n}^{\prime}$ que $\left.f_{n}^{\prime}\right|_{K_{1}}=f_{n}^{\prime} \circ h$ para todo $n \in N_{1}$.

Se $x \in \overline{K_{[0, r[}} \backslash K_{1}$, para cada $n \in N_{1}$, usamos o Lema de Urysohn para encontrar $f_{n}^{\prime}$ contínua tal que:

$$
\left.f_{n}^{\prime}\right|_{B_{n}} \equiv 1,\left.\quad f_{n}^{\prime}\right|_{\left(K \backslash A_{n}^{\prime}\right)} \equiv 0 .
$$

Portanto, $\Delta\left(\left(f_{n}^{\prime}\right)_{n \in N_{1}}\right)=\{x\}$, porque $\left(A_{n}^{\prime}\right)_{n \in N_{1}}$ converge a $x$, e $\left.f_{n}^{\prime}\right|_{K_{1} \cup K_{3}} \equiv 0$, pois $V_{n} \subset K_{[0, r+\sigma[} \backslash$ $K_{1}$, para todo $n \in N_{1}$.

Agora suponhamos que $x \in K_{\left\{\frac{1}{3}\right\}} \cap K_{1}$. Adicionalmente a $\left(V_{n}\right)_{n \in \mathbb{N}}$ considerarmos um sistema fundamental de vizinhanças abertas $\left(V_{n}^{\prime}\right)_{n \in \mathbb{N}}$ de $h(x)$, decrescente, convergindo para $h(x)$, e com $V_{1}^{\prime} \subset K_{] r+\sigma, 1]}$ onde $\sigma$ é definido como acima. Usando o fato que $h$ é homeomorfismo, podemos supor também que $h\left[V_{n} \cap K_{1}\right]=V_{n}^{\prime} \cap K_{3}$.

Novamente achamos sequências crescentes de inteiros positivos $j_{n}$ e $k_{n}$ tais que $V_{k_{1}} \subset K_{[0, r[}$ e $\left|\mu_{j_{n}}\left(A_{j_{n}} \cap V_{k_{n}}\right)\right|>\delta^{\prime}$, e definimos $A_{j_{n}}^{\prime}=A_{j_{n}} \cap V_{k_{n}}$. Fixamos $\delta_{j_{n}}>\delta^{\prime}$ tal que $\left|\mu_{j_{n}}\left(A_{j_{n}}^{\prime}\right)\right|>\delta_{j_{n}}$. Tomamos $B_{j_{n}} \subset A_{j_{n}}^{\prime}$ fechado tal que $\left|\mu_{j_{n}}\left(B_{j_{n}}\right)\right|>\delta_{j_{n}}$ e $\left|\mu_{j_{n}}\right|\left(A_{j_{n}}^{\prime} \backslash B_{j_{n}}\right)<\delta_{j_{n}}-\delta^{\prime}$.

Tomamos $N_{1}^{\prime}=\left\{j_{n}: n \in \mathbb{N}\right\}$. Usando normalidade de $K$, para cada $n \in N_{1}^{\prime}$, achamos $A_{n}^{\prime \prime} \subset A_{n}^{\prime}$ aberto tal que $B_{n} \subset A_{n}^{\prime \prime} \subset \overline{A_{n}^{\prime \prime}} \subset A_{n}^{\prime}$. Para cada $n \in N_{1}^{\prime}$, pelo Lema de Urysohn, existe uma função contínua $g_{n}: K \rightarrow[0,1]$ tal que

$$
\left.g_{n}\right|_{B_{n}} \equiv 1,\left.\quad g_{n}\right|_{K \backslash A_{n}^{\prime \prime}} \equiv 0
$$

Para $n \in N_{1}^{\prime}$, se $B_{n} \cap K_{1}=\emptyset$ então, exigimos $\overline{A_{n}^{\prime \prime}} \subset A_{n}^{\prime} \cap\left(K \backslash K_{1}\right)$ e definimos $f_{n}^{\prime}=g_{n}$. Note que, consequentemente, $\left.f_{n}^{\prime}\right|_{K_{1}}=f_{n}^{\prime} \circ h \equiv 0$. Se $B_{n} \cap K_{1} \neq \emptyset$, como $h\left[\overline{A_{n}^{\prime \prime}} \cap K_{1}\right]$ é fechado em $K$, usamos normalidade de $K$ para achar um aberto $O_{n}$ tal que $h\left[\overline{A_{n}^{\prime \prime}} \cap K_{1}\right] \subset O_{n} \subset \bar{O}_{n} \subset V_{n}^{\prime} \cap A_{n}$. Para cada $n \in N_{1}^{\prime}$, tomamos $\hat{g}_{n}: K \rightarrow[0,1]$ como uma extensão pelo Teorema de Tietze da função $g_{n} \circ h^{-1}: K_{3} \rightarrow[0,1]$ e, usando o Lema de Urysohn, tomamos $\check{g}_{n}: K \rightarrow[0,1]$ tal que

$$
\left.\check{g}_{n}\right|_{h\left[\overline{A_{n}^{\prime \prime}} \cap K_{1}\right]} \equiv 1,\left.\quad \check{g}_{n}\right|_{K \backslash O_{n}} \equiv 0
$$

Assim, definindo $f_{n}^{\prime}=g_{n}+\check{g}_{n} \cdot \hat{g}_{n}$, para cada $n \in N_{1}^{\prime}$, temos $\left.f_{n}^{\prime}\right|_{K_{3}}=f_{n}^{\prime} \circ h$. De fato, sejam $z \in K_{1}$ e $n \in N_{1}^{\prime}$. Se $z \in A_{n}^{\prime \prime} \cap K_{1}$, então $f_{n}^{\prime}(z)=g_{n}(z)$ e $h(z) \in h\left[\overline{A_{n}^{\prime \prime}} \cap K_{1}\right]$. Logo $f_{n}^{\prime}(h(z))=\hat{g}_{n}(h(z))=g_{n}(z)$. Se $z \in K_{1} \backslash A_{n}^{\prime \prime}$, então $g_{n}(z)=0$. Logo $\hat{g}_{n}(h(z))=0$ e, portanto, $f_{n}^{\prime}(h(z))=0$.

Seja $J=\left\{n \in N_{1}^{\prime}: B_{n} \cap K_{1}=\emptyset\right\}$. Se $J$ é infinito, definimos $N_{1}=J$. Caso contrário, definimos $N_{1}=N_{1}^{\prime} \backslash J$. Como $\operatorname{supp}\left(f_{n}^{\prime}\right) \subset A_{n}^{\prime} \cap\left(K \backslash K_{1}\right) \subset V_{n}$, para todo $n \in J$, temos que, se $J$ é infinito,

$$
\operatorname{supp}\left(f_{n}^{\prime}\right) \stackrel{n \in N_{1}}{\longrightarrow} x
$$

e, como $V_{n} \subset K_{[0, r+\sigma[}$, então $\left.f_{n}^{\prime}\right|_{K_{1} \cup K_{3}} \equiv 0$, para todo $n \in N_{1}$. Se $J$ for finito, tomamos $N_{1}=N_{1}^{\prime} \backslash J$ e, assim, $\operatorname{supp}\left(f_{n}^{\prime}\right) \cap K_{1} \stackrel{n \in N_{1}}{\longrightarrow} x$ e $\operatorname{supp}\left(f_{n}^{\prime}\right) \cap K_{3} \stackrel{n \in N_{1}}{\longrightarrow} h(x)$, pois $\left(V_{n}\right)_{n \in \mathbb{N}}$ converge a $x$ e $\left(V_{n}^{\prime}\right)_{n \in \mathbb{N}}$ converge a $h(x)$.

Agora aplicamos o Lema 5.13 a $\left(f_{n}^{\prime}\right)_{n \in N_{1}},\left(\mu_{n}\right)_{n \in N_{1}}, \varepsilon_{n}=\left(\delta_{n}-\delta^{\prime}\right) / 2$. A prova de (b), (c) e da primeira parte de (a) segue imediatamente do lema. Para provar a segunda parte de (a), seja $\left(f_{n}^{\prime \prime}\right)_{n \in N_{1}}$ a sequência de funções obtida pelo Lema 5.13. Portanto, $\left|\int f_{n}^{\prime \prime} d \mu_{n}\right|>\delta^{\prime}$ para todo $n \in N_{1}$.

Aplicando o Lema de Rosenthal para $\delta^{\prime} / 3,\left(\left|\mu_{n}\right|\right)_{n \in N_{1}}$ e a sequência de abertos $\left\{x: f_{n}^{\prime \prime}(x)>0\right\}$, 
dois a dois disjuntos, obtemos um subconjunto infinito $N_{2}$ de $N_{1}$ tal que $\sum\left\{\int f_{m}^{\prime \prime} d\left|\mu_{n}\right|: m \neq n, m \in\right.$ $\left.N_{2}\right\}<\delta / 3$, para todo $n \in N_{2}$.

Agora provaremos (d). Nos casos $x \in K_{[0, r[} \backslash K_{1}$ (subcaso de $x \notin K_{1} \cap K\left\{\frac{1}{3}\right\}$ ) e $x \in K_{1} \cap K\left\{\frac{1}{3}\right\}$ tendo tomado $J=N_{1}$, o fato que $\operatorname{supp}\left(f_{n}^{\prime \prime}\right) \subset \operatorname{supp}\left(f_{n}^{\prime}\right)$ para todo $n \in N_{2} \subset N_{1}$ implica que $\left.f_{n}^{\prime \prime}\right|_{K_{1} \cup K_{3}} \equiv 0$. Além disso, de $\operatorname{supp}\left(f_{n}^{\prime}\right) \stackrel{n \in N_{1}}{\longrightarrow} x$ segue $\operatorname{supp}\left(f_{n}^{\prime}\right) \stackrel{n \in N_{2}}{\longrightarrow} x$. Logo $\Delta\left(\left(f_{n}^{\prime \prime}\right)_{n \in N_{2}}\right)=\{x\}$, acontecendo o caso (II).

Nos casos $x(0)<1 / 3$ e $x \in K_{1} \cap K_{\left\{\frac{1}{3}\right\}}$, com $J$ finito, o fato que $\operatorname{supp}\left(f_{n}^{\prime}\right) \cap K_{1} \stackrel{n \in N_{1}}{\longrightarrow} x$ e $\operatorname{supp}\left(f_{n}^{\prime}\right) \cap K_{3} \stackrel{n \in N_{1}}{\longrightarrow} h(x)$ junto com $\operatorname{supp}\left(f_{n}^{\prime \prime}\right) \subset \operatorname{supp}\left(f_{n}^{\prime}\right)$, para todo $n \in N_{2}$, e a parte (c) implicam $\operatorname{supp}\left(f_{n}^{\prime \prime}\right) \cap K_{1} \stackrel{n \in N_{2}}{\longrightarrow} x$ e $\operatorname{supp}\left(f_{n}^{\prime \prime}\right) \cap K_{3} \stackrel{n \in N_{2}}{\longrightarrow} h(x)$. Como $V_{1} \subset K_{1}$ se $x(0)<1 / 3$ e $N_{1}=N_{1}^{\prime} \backslash J$, se $x \in K_{1} \cap K_{\left\{\frac{1}{3}\right\}}$ e $J$ é finito, então, nesses casos, $\left.f_{n}^{\prime}\right|_{K \backslash\left(K_{1} \cup K_{3}\right)} \equiv 0$, para todo $n \in N_{2}$. Assim, $\Delta\left(\left(f_{n}^{\prime \prime}\right)_{n \in N_{2}}\right)$ verifica (III).

\section{CASO 2. Não acontece caso 1.}

Para todo $n \in \mathbb{N}$ e $\delta^{\prime}>0$, existem $m\left(n, \delta^{\prime}\right) \in \mathbb{N}$ e uma vizinhança aberta $V\left(n, \delta^{\prime}\right)$ de $x_{n}$ tais que $\left|\mu_{k}\right|\left(A_{k} \cap V\left(n, \delta^{\prime}\right)\right)<\delta^{\prime}$, para todo $k>m\left(n, \delta^{\prime}\right)$. Além disso, para cada $n \in \mathbb{N}$ com $x_{n} \in K_{3}$, consideramos $m_{h}(n, \delta) \in \mathbb{N}$ e uma vizinhança aberta $V_{h}\left(n, \delta^{\prime}\right)$ de $h^{-1}\left(x_{n}\right)$ tais que $\left|\mu_{k}\right|\left(A_{k} \cap V_{h}\left(n, \delta^{\prime}\right)\right)<\delta^{\prime}$ para todo $k>m_{h}\left(n, \delta^{\prime}\right)$.

Trocando $V\left(n, \delta^{\prime}\right)$ por $V^{\prime}\left(n, \delta^{\prime}\right) \subset \overline{V^{\prime}\left(n, \delta^{\prime}\right)} \subset V\left(n, \delta^{\prime}\right)$ e, se $x_{n} \in K_{3}, V_{h}\left(n, \delta^{\prime}\right)$ por $V_{h}^{\prime}\left(n, \delta^{\prime}\right) \subset$ $\overline{V_{h}^{\prime}\left(n, \delta^{\prime}\right)} \subset V_{h}\left(n, \delta^{\prime}\right)$, podemos supor que $\left|\mu_{k}\right|\left(A_{k} \cap \overline{V\left(n, \delta^{\prime}\right)}\right)<\delta^{\prime}$, para todo $k>m\left(n, \delta^{\prime}\right)$, e $\left|\mu_{k}\right|\left(A_{k} \cap \overline{V_{h}^{\prime}\left(n, \delta^{\prime}\right)}\right)<\delta^{\prime}$, para todo $k>m_{h}\left(n, \delta^{\prime}\right)$, sempre que $x_{n} \in K_{3}$.

Fixamos $r<\sigma<2 / 3$. Pela propriedade das medidas e a normalidade de $K$ podemos supor que, se $x_{n} \in K \backslash K_{1}$, então $\overline{V\left(n, \delta^{\prime}\right)} \subset K \backslash K_{1}$.

Por indução achamos uma sequência estritamente crescente de inteiros $\left(k_{n}\right)_{n \in \mathbb{N}}$ tais que $k_{n}>$ $m\left(j, \frac{\varepsilon}{2^{j+4}}\right)$, para todos $j<n$ e $k_{n}>\max \left\{m_{h}\left(j, \frac{\varepsilon}{2^{j+4}}\right): j<n, x_{j} \in K_{3}\right\}$.

Seja

$$
A_{k_{n}}^{\prime}=A_{k_{n}} \backslash\left(\bigcup_{j<n} \overline{V\left(j, \varepsilon / 2^{j+4}\right)} \cup \bigcup\left\{\overline{V_{h}\left(j, \varepsilon / 2^{j+4}\right)}: j<n, x_{j} \in K_{3}\right\}\right) .
$$

Temos que $A_{k_{n}}^{\prime}$ é aberto e $h\left[A_{k_{n}}^{\prime} \cap K_{1}\right] \subset A_{k_{n}}$ é aberto em $K_{3}$.

Como $\left|\mu_{k_{n}}\right|\left(A_{k_{n}} \cap \overline{V\left(j, \varepsilon / 2^{j+4}\right)}\right)<\varepsilon / 2^{j+4}$ e $\left|\mu_{k_{n}}\right|\left(A_{k_{n}} \cap \overline{V_{h}\left(j, \varepsilon / 2^{j+4}\right)}\right)<\varepsilon / 2^{j+4}$, para todo $j<n$ (e $x_{j} \in K_{3}$ no segundo caso), temos

$$
\left|\mu_{k_{n}}\left(A_{k_{n}}^{\prime}\right)\right|>\varepsilon / 2
$$

Para cada $n \in \mathbb{N}$ tome $\delta_{k_{n}}>\delta^{\prime}$ tal que $\left|\mu_{k_{n}}\right|\left(A_{K_{n}}^{\prime}\right)>\delta_{k_{n}}$.

Usando a regularidade das medidas tomamos $B_{k_{n}} \subset A_{k_{n}}^{\prime}$ fechado tal que $\left|\mu_{k_{n}}\left(B_{k_{n}}\right)\right|>\delta_{k_{n}}$ e $\left|\mu_{k_{n}}\right|\left(A_{k_{n}}^{\prime} \backslash B_{k_{n}}\right)<\delta_{k_{n}}-\delta^{\prime}$. Usando normalidade de $K$, tome $B_{k_{n}} \subset A_{k_{n}}^{\prime \prime} \subset \overline{A_{k_{n}}^{\prime \prime}} \subset A_{k_{n}}^{\prime}$. Pelas propriedades das medidas podemos supor $\overline{A_{k_{n}}^{\prime \prime}} \subset A_{k_{n}}^{\prime} \cap K_{[0, \sigma[}$.

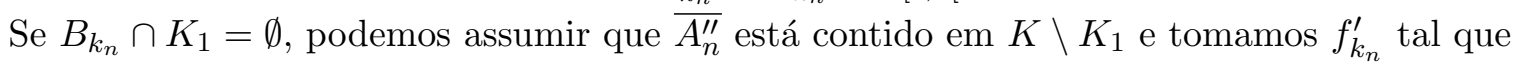

$$
\left.f_{k_{n}}^{\prime}\right|_{B_{k_{n}}} \equiv 1 \text { e }\left.f_{k_{n}}^{\prime}\right|_{K \backslash A_{k_{n}}^{\prime \prime}} \equiv 0
$$

Se $B_{k_{n}} \cap K_{1} \neq \emptyset$, usando o Lema de Urysohn tome $g_{k_{n}}$ tal que

$$
\left.g_{k_{n}}\right|_{B_{k_{n}}} \equiv 1, \text { e }\left.g_{k_{n}}\right|_{K \backslash A_{k_{n}}^{\prime \prime}} \equiv 0
$$


Defina $\hat{g}_{k_{n}}: K \rightarrow[0,1]$ como uma extensão, pelo Teorema de Tietze, da função $g_{k_{n}} \circ h^{-1}: K_{3} \rightarrow$ $[0,1]$.

Note que $h\left[\overline{A_{k_{n}}^{\prime \prime}} \cap K_{1}\right]$ é fechado em $K_{3}$ e $h\left[B_{k_{n}} \cap K_{1}\right] \subset h\left[A_{k_{n}}^{\prime} \cap K_{1}\right] \subset h\left[\overline{A_{k_{n}}^{\prime \prime}} \cap K_{1}\right] \subset h\left[A_{k_{n}}^{\prime} \cap K_{1}\right] \subset$ $A_{k_{n}} \cap K_{3}$. Como $h\left[A_{k_{n}}^{\prime \prime} \cap K_{1}\right]$ é aberto em $K_{3}$, existe $\tilde{U}_{k_{n}} \subset K_{] \sigma, 1]} \cap A_{k_{n}}$ aberto em $K$ tal que $\tilde{U}_{k_{n}} \cap K_{3}=h\left[A_{k_{n}}^{\prime} \cap K_{1}\right]$, para cada $n \in \mathbb{N}$. Agora, para cada $j \in \mathbb{N}$ achamos abertos $\tilde{V}_{j} \subset V\left(j, \varepsilon / 2^{j+4}\right)$ . Se $x_{j} \in K_{[0, \sigma[} \cup K_{] 2 / 3,1]}$, ou $x_{j} \in A_{n}$, para algum $n \in \mathbb{N}$, escolhemos $\tilde{V}_{j}=\emptyset$. No caso contrário, se $x_{j} \in K_{[\sigma, 2 / 3]} \backslash K_{3}$, escolhemos $\tilde{V}_{j}$ de modo que $x_{j} \in \tilde{V}_{j} \subset \overline{\tilde{V}_{j}} \subset V\left(j, \varepsilon / 2^{j+4}\right) \cap\left(K \backslash K_{3}\right)$. Se $x_{j} \in K_{3} \cap K_{\left\{\frac{2}{3}\right\}}$, escolhemos $\tilde{V}_{j}$ de modo que $x_{j} \in \tilde{V}_{j} \cap K_{3} \subset h\left[V_{h}\left(j, \varepsilon / 2^{4+j}\right) \cap K_{1}\right]$. Pela normalidade de $K$, para todo $n \in N$, existe $U_{k_{n}}$ aberto em $K$ tal que $h\left[\overline{A_{k_{n}}^{\prime \prime}} \cap K_{1}\right] \subset U_{k_{n}} \subset \overline{U_{k_{n}}} \subset \tilde{U}_{k_{n}} \backslash \bigcup_{j<n} \overline{\tilde{V}_{j}}$. Note que, como $\tilde{V}_{j} \subset V\left(j, \varepsilon / 2^{j+4}\right)$, então

$$
A_{k_{n}} \cap \tilde{V}_{j} \text {, para todo } n>j .
$$

Novamente, usando o Lema de Urysohn encontramos $\check{g}_{n}: K \rightarrow[0,1]$ tal que

$$
\left.\check{g}_{k_{n}}\right|_{h\left[\overline{A_{k_{n}}^{\prime \prime}} \cap K_{1}\right]} \equiv 1, \text { e }\left.\check{g}_{k_{n}}\right|_{K \backslash U_{k_{n}}} \equiv 0
$$

Finalmente, defina $f_{k_{n}}^{\prime}=g_{k_{n}}+\hat{g}_{k_{n}} \cdot \check{g}_{k_{n}}$. Vejamos que $f_{k_{n}}^{\prime}(x)=f_{k_{n}}^{\prime}(h(x))$, para todo $x \in K_{1}$ e todo $n \in \mathbb{N}$. Se $x \in\left(K \backslash A_{k_{n}^{\prime \prime}}\right) \cap K_{1}$, então $f_{k_{n}}^{\prime}(x)=0$ e $h(x) \in K_{3} \backslash h\left[A_{k_{n}}^{\prime \prime} \cap K_{1}\right]$. Logo $\hat{g}_{k_{n}}(h(x))=0 \mathrm{e}$, portanto, $f_{k_{n}}^{\prime}(h(x))=0$. Se $x \in A_{k_{n}}^{\prime \prime} \cap K_{1}$, então $f_{k_{n}}^{\prime}(x)=g_{k_{n}}(x), \check{g}_{k_{n}}(h(x))=1 \mathrm{e}$ $\hat{g}_{k_{n}}(h(x))=g_{k_{n}}(x)$. Portanto, $f_{k_{n}}^{\prime}(h(x))=g_{k_{n}}(x)$.

Note que, se $x_{j} \in A_{n}$, para algum $n \in \mathbb{N}$, então $x_{j} \notin \Delta\left(f_{k_{n}}^{\prime}\right)_{n \in \mathbb{N}}$, porque os abertos $A_{n}$ são disjuntos dois a dois. Por outro lado, como $V\left(j, \varepsilon / 2^{j+4}\right)$ é disjunto de $A_{k_{n}}^{\prime}$, para $n>j$, então, para qualquer $j \in \mathbb{N}$,

$$
x_{j} \notin \Delta\left(\left(A_{k_{n}}^{\prime}\right)_{n \in \mathbb{N}}\right) .
$$

Como $\operatorname{supp}\left(f_{k_{n}}\right) \cap K_{[0, \sigma[} \subset \overline{A_{k_{n}}^{\prime \prime}} \subset A_{k_{n}}^{\prime}$, para todo $n \in \mathbb{N}$, então $x_{j} \in K_{[0, \sigma[}$ o aberto $V\left(j, \varepsilon / 2^{j+4}\right) \cap$ $K_{[0, \sigma[}$ contém $x_{j}$ e intersecta no máximo um número finito de suportes de funções $f_{k_{n}}$. Portanto, se $x_{j} \in K_{[0, \sigma[}$, então $x_{j} \notin \Delta\left(\left(f_{k_{n}}^{\prime}\right)_{n \in \mathbb{N}}\right)$.

Se $x_{j} \in K_{[\sigma, 1]} \backslash K_{3}$ e $x_{j} \notin A_{n}$, para todo $n \in \mathbb{N}$, então $\tilde{V}_{j}$ é disjunto de $U_{k_{n}}$, para todo $n>j$. Logo, $x_{j} \notin \Delta\left(U_{k_{n}}\right)_{n \in \mathbb{N}}$, o que, junto com (5.11), implica $x_{j} \notin \Delta\left(\left(f_{k_{n}}^{\prime}\right)_{n \in \mathbb{N}}\right)$, sempre que $x_{j} \in K_{[\sigma, 1]} \backslash K_{3}$.

Se $x_{j} \in K_{3}$, então $h\left[V_{h}\left(j, \varepsilon / 2^{j+4}\right) \cap K_{1}\right]$ é disjunto de $h\left[A_{k_{n}}^{\prime} \cap K_{1}\right]$, para todo $n>j$. Se $x_{j}(0)>2 / 3$, então $h\left[V_{h}\left(j, \varepsilon / 2^{j+4}\right) \cap K_{\left[0, \frac{1}{3}[\right.}\right]$ é um aberto de $K$ contendo $x_{j}$ e disjunto de $h\left[A_{k_{n}}^{\prime} \cap K_{1}\right]$. Portanto, $x_{j} \notin \Delta\left(\left(f_{k_{n}}^{\prime}\right)_{n \in \mathbb{N}}\right)$. Se $x_{j} \in K_{3}$ e $x_{j}(0)=2 / 3$, então $\tilde{V}_{j} \cap U_{k_{n}}=\emptyset$, para todo $n>j$. Portanto, $x_{j} \notin \Delta\left(\left(U_{k_{n}}\right)_{n \in \mathbb{N}}\right)$. Como $\operatorname{supp}\left(f_{k_{n}}\right) \cap K_{] \sigma, 1]} \subset \overline{U_{k_{n}}}$ e, neste caso, $\tilde{V}_{j} \subset K_{] \sigma, 1]}$, temos $x_{j} \notin \Delta\left(\left(f_{k_{n}}^{\prime}\right)_{n \in \mathbb{N}}\right)$.

Definindo $N_{1}:=\left\{k_{n}: n \in \mathbb{N}\right\}$, temos $x_{m} \notin \Delta\left(\left(f_{n}^{\prime}\right)_{n \in N_{1}}\right)$, para todo $m \in \mathbb{N}, h\left(x_{m}\right) \notin$ $\Delta\left(\left(f_{n}^{\prime}\right)_{n \in N_{1}}\right)$, se $x_{m} \in K_{1}$, e $h^{-1}\left(x_{m}\right) \notin \Delta\left(\left(f_{n}^{\prime}\right)_{n \in N_{1}}\right)$, se $x_{m} \in K_{3}$.

Note que

$$
\left|\int f_{n}^{\prime} d \mu_{n}\right|=\left|\int_{B_{n}} f_{n}^{\prime} d \mu_{n}+\int_{A_{n}^{\prime \prime} \backslash B_{n}} f_{n}^{\prime} d \mu_{n}\right|>|| \int_{B_{n}} f_{n}^{\prime} d \mu_{n}|-| \int_{A_{n}^{\prime \prime} \backslash B_{n}} f_{n}^{\prime} d \mu_{n} \|>\delta
$$

Agora aplicamos o Lema 5.13 a $\left(f_{n}^{\prime}\right)_{n \in N_{1}},\left(\mu_{n}\right)_{n \in N_{1}}, \varepsilon_{n}=\left(\delta_{n}-\delta\right) / 2$. A prova de (b) e (c) 
seguem imediatamente do lema, a primeira parte de (a) segue de (5.12) e da parte (g) do lema. Para provar a segunda parte de (a), seja $\left(f_{n}^{\prime \prime}\right)_{n \in N_{1}}$ a sequência de funções obtida de aplicar o Lema 5.13. Temos $\left|\int f_{n}^{\prime \prime} d \mu_{n}\right|>\delta$, para todo $n \in N_{1}$. Aplicando o Lema de Rosenthal para $\delta / 3,\left(\left|\mu_{n}\right|\right)_{n \in N_{1}}$ e a sequência de abertos $\left\{x: f_{n}^{\prime \prime}(x)>0\right\}$ dois a dois disjuntos, obtemos um subconjunto infinito $N_{2}$ de $N_{1}$ tal que $\sum\left\{\int f_{m}^{\prime \prime} d\left|\mu_{n}\right|: m \neq n, m \in N_{2}\right\}<\delta / 3$, para todo $n \in N_{2}$. A prova de que $\Delta\left(\left(f_{n}^{\prime \prime}\right)_{n \in N_{2}}\right)$ verifica o caso (I) de (d) é consequência de que $\operatorname{supp}\left(f_{n}^{\prime \prime}\right) \subset \operatorname{supp}\left(f_{n}^{\prime}\right)$. A prova do lema é completada apenas tomando as funções $f_{n}^{\prime}$ do enunciado do lema como as $f_{n}^{\prime \prime}$ para $n \in N_{2}$.

O seguinte lema - cuja prova é baseada na do Teorema 5.1 de [Faj12] - será usado para definir o passo sucessor na construção indutiva que faremos no Capítulo 7.

Lema 5.15. Sejam $K \subset[0,1]^{\gamma}$ um espaço metrizável, compacto e sem pontos isolados, com $\gamma<\omega_{1}$, e $Q=\left\{q_{n}: n \in \mathbb{N}\right\}$ um conjunto denso em $K$, e $1 / 3<r<2 / 3$. Defina $K_{1}=\overline{K_{\left[0, \frac{1}{3}[\right.}}$ e $K_{3}=\overline{K_{\left.] \frac{2}{3}, 1\right]}}$ e suponha que a função $h: K_{1} \rightarrow K_{3}$ definida por $h(x)(0)=1-x(0)$ e $h(x)(\alpha)=x(\alpha)$ para $\alpha>0$, está bem definida, é bijetora e contínua (e, portanto, um homeomorfismo entre $K_{1}$ e $K_{3}$ ). Dados:

(a) uma sequência $\left(f_{n}: n \in \mathbb{N}\right)$ de funções contínuas de $K$ em $[0,1]$, duas a duas disjuntas, tais que $f_{n}(h(x))=f_{n}(x)$ para todo $x \in K_{1}$ e todo $n \in \mathbb{N}$;

(b) uma sequência crescente $\left(l_{n}\right)_{n \in \mathbb{N}}$ em $\mathbb{N}$ tal que $\left\{q_{l_{n}}: n \in \mathbb{N}\right\}$ é relativamente discreta e $f_{m}\left(q_{l_{n}}\right)=0$, para todos $n, m \in \mathbb{N}$;

(c) um conjunto enumerável $\mathcal{P}$ de pares $(L, R) \in(\mathcal{P}(\mathbb{N}))^{2}$ tais que $L \cap R=\emptyset$ e $\overline{\left\{q_{n}: n \in L\right\}} \cap$ $\overline{\left\{q_{n}: n \in R\right\}} \neq \emptyset$, em $K$;

(d) $u m \varepsilon>0$;

(e) uma sequência limitada $\left(\mu_{n}: n \in \mathbb{N}\right)$ de medidas regulares sobre $K$ tais que, para todo $n \in \mathbb{N}$, $\left|\int f_{n} d \mu_{n}\right|>\varepsilon$ e $\left|\mu_{n}\right|\left(K \backslash \overline{\left.K_{] r, 1]}\right)}=0\right.$, para todo $n \in \mathbb{N}$, ou $\left|\mu_{n}\right|\left(K \backslash \overline{\left.K_{[0, r[}\right)}=0\right.$, para todo $n \in \mathbb{N}$;

existem $\delta>0, b^{\prime} \subset a \subset \mathbb{N}$ infinitos e funções contínuas $f_{n}^{\prime}: K \longrightarrow[0,1]$ tais que $\operatorname{supp}\left(f_{n}^{\prime}\right) \subset$ $\operatorname{supp}\left(f_{n}\right)$ e, para todo $b \subseteq b^{\prime}$, temos:

(f) $\left|\int f_{n}^{\prime} d \mu_{n}\right|>\delta$ e $\Sigma\left\{\int f_{m}^{\prime} d\left|\mu_{n}\right|: m \neq n, m \in a\right\}<\delta / 3$, para todo $n \in a$;

(g) $L=K\left(\left(f_{n}^{\prime}\right)_{n \in b}\right)$ é uma extensão completa;

(h) $\overline{\left\{q_{l_{n}}^{\prime}: n \in b\right\}} \cap \overline{\left\{q_{l_{n}}^{\prime}: n \in a \backslash b\right\}} \neq \emptyset$, onde $q_{m}^{\prime}$ é definido como $\left(q_{m}, \Sigma_{n \in b} f_{n}^{\prime}\left(q_{m}\right)\right)$;

(i) $\overline{\left\{q_{n}^{\prime}: n \in L\right\}} \cap \overline{\left\{q_{n}^{\prime}: n \in R\right\}} \neq \emptyset$, para todo $(L, R) \in \mathcal{P}$;

(j) $\left.f_{n}^{\prime}\right|_{K_{1}}=f_{n}^{\prime} \circ h$

Demonstração: Assumimos $\left|\mu_{n}\right|\left(K \backslash \overline{K_{] r, 1]}}\right)=0$, sendo a prova do outro caso análoga.

Para cada $(L, R) \in \mathcal{P}$, escolhemos $z_{L R} \in \overline{\left\{q_{n}: n \in L\right\}} \cap \overline{\left\{q_{n}: n \in R\right\}}$. Assim, o conjunto $Z:=$ $\left\{q_{l_{n}}: n \in \mathbb{N}\right\} \cup\left\{z_{L R}:(L, R) \in \mathcal{P}\right\}$ é enumerável.

Aplicando o Lema 5.14 para $\left(f_{n}\right)_{n \in \mathbb{N}}, \varepsilon,\left(\mu_{n}\right)_{n \in \mathbb{N}}$, como no enunciado do lema, e tomando $\left(x_{n}\right)_{n \in \mathbb{N}}$ como uma enumeração do conjunto $Z$, achamos $\delta>0, a \subset \mathbb{N}$ infinito e funções contínuas 
$f_{n}^{\prime}: K \rightarrow[0,1], \operatorname{com} \operatorname{supp}\left(f_{n}^{\prime}\right) \subset \operatorname{supp}\left(f_{n}\right)$, tais que $(\mathrm{f}),(\mathrm{g})$ e $(\mathrm{j})$ são verdadeiros para todo $b \subset a$, e acontece exatamente uma das possibilidades (I)-(III) de (d) no Lema 5.14. Para demonstrar (h) e (i) separamos nos mesmos casos de (d) do Lema 5.14.

Se $\Delta\left(\left(f_{n}^{\prime}\right)_{n \in b}\right)$ verifica (I) ou (II) de (d) no Lema 5.14 a prova é exatamente como no caso 1 e caso 2 na prova do Lema 5.16, respectivamente.

Caso I: $\Delta\left(\left(f_{n}^{\prime}\right)_{n \in a}\right) \cap Z=\emptyset$.

Para cada $(L, R) \in \mathcal{P}$ existe uma vizinhança aberta de $z_{L R}$ que intersecta apenas um número finito de suportes das funções $f_{n}^{\prime}$. Logo, $K\left(\left(f_{n}^{\prime}\right)_{n \in a}\right)$ é localmente o gráfico de uma função contínua nesse ponto e, portanto, $\left(z_{L R}, \sum_{n \in a} f_{n}^{\prime}\left(z_{L R}\right)\right) \in \overline{\left\{q_{n}^{\prime}: n \in L\right\}} \cap \overline{\left\{q_{n}^{\prime}: n \in R\right\}}$. Isso também acontece para qualquer extensão de $K$ por um subconjunto infinito de funções de $\left\{f_{n}^{\prime}: n \in a\right\}$, o que prova (i) para este caso.

Para demonstrar (h), como $K$ é compacto e metrizável, $\left\{q_{l_{n}}: n \in a\right\}$ tem uma subsequência convergente e, portanto, existe $b^{\prime} \subset a$ infinito e co-infinito tal que $\overline{\left\{q_{l_{n}}: n \in b\right\}} \cap \overline{\left\{q_{l_{n}}: n \in a \backslash b\right\}} \neq \emptyset$, para todo $b \subset b^{\prime}$ infinito, e $\left(q_{l_{n}}\right)_{n \in b^{\prime}}$ converge. Seja $z=\lim _{n \in b^{\prime}} q_{l_{n}}$.

Como $f_{m}^{\prime}\left(q_{l_{n}}\right)=0$, para todo $m, n \in \mathbb{N}$, temos $\sum_{m \in a}^{n \in b^{\prime}} f_{m}\left(q_{l_{n}}\right)=0$, para todo $n \in a$. Como $\left\{q_{l_{n}}: n \in \mathbb{N}\right\} \subset Z$, então para cada $n \in \mathbb{N}$ existe uma vizinhança aberta $W_{n}$ de $q_{l_{n}}$ tal que $W_{n}$ intersecta no máximo um número finito de suportes das funções $f_{m}^{\prime}$. Logo,

$$
\left(q_{l_{n}}, 0\right) \in \operatorname{Gr}\left(\left.\left(\sum_{m \in a} f_{m}^{\prime}\right)\right|_{D\left(\left(f_{m}^{\prime}\right)_{m \in a}\right)}\right),
$$

para todo $n \in \mathbb{N}$. O mesmo acontece se trocarmos $a$ por qualquer $b \subset a$ infinito e, em particular, para cada $b \subset b^{\prime}$. Pela definição de $z$, para todo $b \subset b^{\prime}$ temos $\lim _{n \in b}\left(q_{l_{n}}, 0\right)=(z, 0)$, em $K \times[0,1]$, de onde segue que $(z, 0) \in K\left(\left(f_{n}^{\prime}\right)_{n \in b}\right)$. Em particular, para $b \subset b^{\prime}$,

$$
\overline{\left\{q_{l_{n}}^{\curlyvee} 0: n \in b\right\}} \cap \overline{\left\{\widetilde{q_{l_{n}}} 0: n \in a \backslash b\right\}} \neq \emptyset,
$$

com o fecho tomado em $K\left(\left(f_{n}^{\prime}\right)_{n \in b}\right)$, para todo $b \subset b^{\prime}$, provando (h) para este caso.

Caso II: $\Delta\left(\left(f_{n}^{\prime}\right)_{n \in a}\right)$ é unitário.

Enumeramos $\mathcal{P}$ por $\left\{\left(L_{m}, R_{m}\right): m \in \mathbb{N}\right\}$. Como $L_{m}$ e $R_{m}$ são enumeráveis, usando bijeções definimos $Q_{L_{m}}$ como uma enumeração do conjunto $\left\{q_{n}: n \in L_{m}\right\}$ com os ímpares e $Q_{R_{m}}$ como uma enumeração do conjunto $\left\{q_{n}: n \in R_{m}\right\}$ com os pares. Temos, assim, $Q_{L_{m}}=\left\{q_{n m}: n \in \mathbb{N} \backslash 2 \mathbb{N}\right\}=$ $\left\{q_{n}: n \in L_{m}\right\}$ e $Q_{R_{m}}=\left\{q_{n m}: n \in \mathbb{N}\right\}=\left\{q_{n}: n \in R_{m}\right\}$, para cada $m \in \mathbb{N}$. Aplicamos o Lema 5.10 a $\left(f_{n}^{\prime}\right)_{n \in a}$ e os conjuntos $Q_{L_{m}}$ e $Q_{R_{m}}$ obtemos $b^{\prime} \subset a$ que verifica (i).

Para provar (h), como $K$ é metrizável e compacto, então existe uma subsequência convergente de $\left\{q_{l_{n}}: n \in b^{\prime}\right\}$, e portanto, existe $b^{\prime \prime} \subset b^{\prime}$ infinito tal que $\lim _{n \in b^{\prime \prime}} q_{l_{n}}$ existe e pertence ao fecho de $\left\{q_{l_{n}}>n \in b^{\prime} \backslash b^{\prime \prime}\right\}$. Isto acontece para todo $b \subset b^{\prime \prime}$. Seja $z=\lim _{n \in b^{\prime \prime}} q_{l_{n}}$.

Como $\Delta\left(\left(f_{n}^{\prime}\right)_{n \in a}\right)$ é unitário e $\left\{q_{l_{n}}: n \in \mathbb{N}\right\}$ é relativamente discreto, temos $q_{l_{n}} \notin \Delta\left(\left(f_{n}^{\prime}\right)_{n \in a}\right)$, para todo $n \in a$, exceto possivelmente por um único $n_{0}$ (caso $\left\{q_{l_{n_{0}}}\right\}=\Delta\left(\left(f_{n}^{\prime}\right)_{n \in a}\right)$ ). Assim, para cada $n \in b^{\prime \prime}$, diferente de $n_{0}$, existe um aberto $V_{n}$ que intersecta no máximo um número finito de suportes da funções $f_{n}^{\prime}$. Raciocinando como no caso I, provamos que para qualquer $b \subset b^{\prime \prime}$ infinito acontece $(z, 0) \in K\left(\left(f_{n}^{\prime}\right)_{n \in b}\right)$ e $(z, 0) \in \overline{\left\{\widetilde{q_{l_{n}}} 0: n \in b\right\}} \cap \overline{\left\{q_{l_{n}} 0: n \in a \backslash b\right\}} \neq \emptyset$, onde o fecho é tomado em $K\left(\left(f_{n}^{\prime}\right)_{n \in b}\right)$, provando (h) para este caso. 
Caso III: para todo $b \subset a, \Delta\left(\left(f_{n}^{\prime}\right)_{n \in b}\right)=\{x, h(x)\} \operatorname{com} x \in K_{1}$ e, para algum $r<s<2 / 3$,

$$
\operatorname{supp}\left(f_{n}^{\prime}\right) \cap K[0, r] \stackrel{n \in b}{\longrightarrow} x \quad \text { e } \quad \operatorname{supp}\left(f_{n}^{\prime}\right) \cap K_{[s, 1]} \stackrel{n \in b}{\longrightarrow} h(x)
$$

Para provar (i), aplicamos o Lema 5.11 a $\left(f_{n}^{\prime}\right)_{n \in a}, O_{1}=K_{[0,1 / 3[}, O_{2}=K_{] 2 / 3,1]}, O_{1}^{\prime}=K_{[0, r}$, $K_{] s, 1]}, h$ e $\mathcal{P}$. Portanto, existe um subconjunto infinito $b^{\prime}$ de $a$ tal que, para todo $b \subset b^{\prime}$ infinito, acontece

$$
\overline{\left\{q_{n}^{\prime}: n \in L\right\}} \cap \overline{\left\{q_{n}^{\prime}: n \in R\right\}} \neq \emptyset,
$$

onde $q_{n}^{\prime}=\left(q_{n}, \sum_{m \in b} f_{m}\left(q_{n}\right)\right)$ e o fecho é tomado em $K\left(\left(f_{n}\right)_{n \in b}\right)$, para todo $(L, R) \in \mathcal{P}$, provando (i).

Agora demonstramos (h). Como $\left(q_{l_{n}}: n \in b^{\prime}\right)$ é uma sequência infinita e $K$ é compacto e metrizável, então existe uma subsequência convergente. Seja $y$ o limite. Logo, podemos achar $b^{\prime \prime} \subset b^{\prime}$ infinito, tal que $q_{l_{n}} \stackrel{n \in b^{\prime \prime}}{\longrightarrow} y$ e $q_{l_{n}} \stackrel{n \in b^{\prime} \backslash b^{\prime \prime}}{\longrightarrow} y$.

Por (b), o conjunto $\left\{q_{l_{n}}: n \in b^{\prime}\right\}$ é relativamente discreto. Como $\left|\left\{q_{l_{n}}: n \in b^{\prime}\right\} \cap \Delta\left(\left(f_{n}^{\prime}\right)_{n \in b^{\prime \prime}}\right)\right| \leq$ 2 , então para todos exceto possivelmente um par de $n$ 's em $b^{\prime}$ existem abertos $U_{l_{n}}$ 's contendo $q_{l_{n}}$ e que intersectam no máximo um número finito de suportes das funções $f_{n}^{\prime}$ para $n \in b^{\prime}$. Portanto, se $b \subset b^{\prime \prime}$, então $K\left(\left(f_{n}^{\prime}\right)_{n \in b}\right)$ é localmente o gráfico de uma função em $q_{l_{n}}$ para todos exceto possivelmente um par de $n$ 's em $b^{\prime}$. Assim, usando (b), para cada $b \subset b^{\prime \prime},\left(q_{l_{n}}, 0\right) \in K\left(\left(f_{n}^{\prime}\right)_{n \in b}\right)$ para infinitos $n$ 's em $b$ e infinitos $n$ 's em $b^{\prime} \backslash b^{\prime \prime}$ que indexam a subsequência convergente a $y$. Logo, se $b \subset b^{\prime \prime}$ infinito, como $q_{l_{n}} \stackrel{n \in b^{\prime \prime}}{\longrightarrow} y$, então $\left.(y, 0) \in K\left(\left(f_{n}^{\prime}\right)_{n \in b}\right)\right)$ e $\overline{\left\{q_{l_{n}}^{\prime}: n \in b\right\}} \cap \overline{\left\{q_{l_{n}}^{\prime}: n \in a \backslash b\right\}} \neq \emptyset$, com o fecho como sendo tomado em $K\left(\left(f_{n}^{\prime}\right)_{n \in b}\right)$. Assim (h) se verifica para $b^{\prime \prime}$.

Finalmente, como $b^{\prime \prime} \subset b^{\prime} \subset a$, então para todo $b \subset b^{\prime \prime}$ se verificam (h) e (i) neste caso.

Quando se prescindir do homeomorfismo $h$ e em particular da propriedade (j) no lema anterior, é possível obter um resultado similar, onde as medidas de Radon não necessariamente devem se anular numa parte do espaço $K$. O próximo lema apresenta este fato, que será usado no Capítulo 6 . Embora o próximo lema não é um caso particular do Lema 5.15, a prova é praticamente a mesma apresentada para os casos I e II do Lema 5.15 usando o Lema 3.10 de [Faj12] no lugar do Lema 5.15 , pelo que não a escreveremos aqui.

Lema 5.16. Sejam $K \subset[0,1]^{\gamma}$ um espaço metrizável, compacto e sem pontos isolados, com $\gamma<\omega_{1}$, e $Q=\left\{q_{n}: n \in \mathbb{N}\right\}$ um conjunto denso em K. Dados:

(a) uma sequência $\left(f_{n}: n \in \mathbb{N}\right)$ de funções contínuas de $K$ em $[0,1]$, duas a duas disjuntas;

(b) uma sequência crescente $\left(l_{n}\right)_{n \in \mathbb{N}}$ em $\mathbb{N}$ tal que $\left\{q_{l_{n}}: n \in \mathbb{N}\right\}$ é relativamente discreta e $f_{m}\left(q_{l_{n}}\right)=0$, para todos $n, m \in \mathbb{N}$;

(c) um conjunto enumerável $\mathcal{P}$ de pares $(L, R) \in(\mathcal{P}(\mathbb{N}))^{2}$ tais que $L \cap R=\emptyset$ e $\overline{\left\{q_{n}: n \in L\right\}} \cap$ $\overline{\left\{q_{n}: n \in R\right\}} \neq \emptyset$, em $K$;

(d) $u m \varepsilon>0$;

(e) uma sequência limitada $\left(\mu_{n}: n \in \mathbb{N}\right)$ de medidas regulares sobre $K$ tais que, para todo $n \in \mathbb{N}$, $\left|\int f_{n} d \mu_{n}\right|>\varepsilon ;$ 
existem $\delta>0, b^{\prime} \subset a \subset \mathbb{N}$ infinitos e funções contínuas $f_{n}^{\prime}: K \longrightarrow[0,1]$ tais que $\operatorname{supp}\left(f_{n}^{\prime}\right) \subset$ $\operatorname{supp}\left(f_{n}\right)$ e, para todo $b \subseteq b^{\prime}$, temos:

(f) $\left|\int f_{n}^{\prime} d \mu_{n}\right|>\delta$ e $\Sigma\left\{\int f_{m}^{\prime} d\left|\mu_{n}\right|: m \neq n, m \in a\right\}<\delta / 3$, para todo $n \in a$;

(g) $L=K\left(\left(f_{n}^{\prime}\right)_{n \in b}\right)$ é uma extensão completa;

(h) $\overline{\left\{q_{l_{n}}^{\prime}: n \in b\right\}} \cap \overline{\left\{q_{l_{n}}^{\prime}: n \in a \backslash b\right\}} \neq \emptyset$, onde $q_{m}^{\prime}$ é definido como $\left(q_{m}, \Sigma_{n \in b} f_{n}^{\prime}\left(q_{m}\right)\right)$;

(i) $\overline{\left\{q_{n}^{\prime}: n \in L\right\}} \cap \overline{\left\{q_{n}^{\prime}: n \in R\right\}} \neq \emptyset$, para todo $(L, R) \in \mathcal{P}$;

Anteriormente, os resultados, sob algumas condições, garantiam que propriedades de um espaço compacto eram transferidas para uma extensão por funções contínuas ou dos espaços de um sistema inverso para o limite. Agora, no sentido contrário, consideramos conjuntos enumeráveis com fechos não disjuntos no limite de um sistema inverso como o do Lema 5.4, e olhamos para as intersecções dos fechos das imagens pelas projeções do sistema.

Lema 5.17. Sejam $K_{\alpha} \subset[0,1]^{\alpha}$ espaços compactos, para $\alpha<\omega_{1}$, e $K=\lim _{\leftarrow}\left(K_{\alpha}, \pi_{\alpha, \beta}, \omega_{1}\right)=$ $\left\{x \in[0,1]^{\omega_{1}}:\left.x\right|_{\alpha} \in K_{\alpha}\right\}$ compacto separável, com $Q:=\left\{q_{n}: n \in \mathbb{N}\right\}$ como subconjunto denso enumerável. Seja $\pi_{\alpha}$ a projeção canônica de $K$ sobre $K_{\alpha}$, para cada $\alpha$. Se $\overline{\left\{q_{n}: n \in L\right\}} \cap \overline{\left\{q_{n}: n \in R\right\}}=$ $\emptyset$, onde $L, R$ são subconjuntos infinitos de $\mathbb{N}$ disjuntos, então existe $\gamma<\omega_{1}$ tal que, para $\gamma<\alpha<\omega_{1}$, acontece $\overline{\left\{\pi_{\alpha}\left(q_{n}\right): n \in L\right\}} \cap \overline{\left\{\pi_{\alpha}\left(q_{n}\right): n \in R\right\}}=\emptyset$.

Demonstração: Supondo que $\overline{\left\{q_{n}: n \in L\right\}} \cap \overline{\left\{q_{n}: n \in R\right\}}=\emptyset$, pela normalidade do $K$ existem abertos disjuntos $W_{1}$ e $W_{2}$ tais que $\overline{\left\{q_{n}: n \in L\right\}} \subset W_{1}$ e $\overline{\left\{q_{n}: n \in R\right\}} \subset W_{2}$.

Para cada $t \in \overline{\left\{q_{n}: n \in L\right\}}$ seja $U_{t}$ um aberto básico da topologia em $K$ contido em $W_{1}$. Pela compacidade, existe uma coleção finita $\mathcal{A}_{1}$ de abertos $U_{t}$ 's que é um recobrimento finito de $\overline{\left\{q_{n}: n \in L\right\}}$. Por outro lado, como $K$ é separável, existe uma coleção enumerável $\mathcal{C}_{1}$ de abertos básicos de $K$ contidos em $W_{1}$ tais que o fecho da união é igual ao fecho de $W_{1}$. Pela Proposição 1.1, podemos escrever $\mathcal{A}_{1} \cup \mathcal{C}_{1}=\left\{\pi_{\alpha_{n}}^{-1}\left[B_{\alpha_{n}}\right]: n \in \mathbb{N}\right\}$, onde $B_{\alpha_{n}}$ é um aberto em $K_{\alpha_{n}}$, e, portanto, denotando $U_{1}=\bigcup_{n \in \mathbb{N}} \pi_{\alpha_{n}}^{-1}\left[B_{\alpha_{n}}\right]$, temos

$$
\overline{\left\{q_{n}: n \in L\right\}} \subset U_{1} \subset W_{1}
$$

Analogamente, existe uma união $U_{2}$ de abertos básicos de $K$ contidos em $W_{2}$ tal que $\overline{\left\{q_{n}: n \in R\right\}} \subset$ $U_{2} \subset W_{2}$. Em consequência, pela Proposição 1.3 existe $\gamma<\omega_{1}$ tal que, para todo $\alpha>\gamma$, acontece $\pi_{\alpha}^{-1}\left[\pi_{\alpha}\left[U_{1}\right]\right]=U_{1}$ e $\pi_{\alpha}^{-1}\left[\pi_{\alpha}\left[U_{2}\right]\right]=U_{2}$. Portanto, $\pi_{\alpha}\left[U_{1}\right]$ e $\pi_{\alpha}\left[U_{2}\right]$ são disjuntos em $K_{\alpha}$ pois $U_{1} \cap U_{2}=\emptyset$.

Como $K$ e $K_{\alpha}$ são compactos Hausdorff e $\pi_{\alpha}$ contínua, então $\pi_{\alpha}\left[\overline{\left\{q_{n}: n \in L\right\}}\right]$ e $\pi_{\alpha}\left[\overline{\left\{q_{n}: n \in R\right\}}\right]$ são fechados em $K_{\alpha}$. Portanto,

$$
\left\{\pi_{\alpha}\left(q_{n}\right): n \in L\right\} \subset \pi_{\alpha}\left[\overline{\left\{q_{n}: n \in L\right\}}\right] \subset \pi_{\alpha}\left[U_{1}\right]
$$

e

$$
\left\{\pi_{\alpha}\left(q_{n}\right): n \in R\right\} \subset \pi_{\alpha}\left[\overline{\left\{q_{n}: n \in R\right\}}\right] \subset \pi_{\alpha}\left[U_{2}\right]
$$

implicam 


$$
\overline{\left\{\pi_{\alpha}\left(q_{n}\right): n \in L\right\}} \cap \overline{\left\{\pi_{\alpha}\left(q_{n}\right): n \in R\right\}}=\emptyset
$$

Nas construções indutivas de espaços fracamente Koszmider, desde a primeira, feita em [Kos04], é essencial que as funções contínuas definidas no espaço todo sejam totalmente determinadas a partir de um passo intermediário. Para isso, usa-se o Teorema de Mibu.

Teorema 5.18 (Mibu, [Mib]). Se $f$ é uma função contínua definida de um produto infinito de espaços completamente regulares em $\mathbb{R}$, então $f$ é determinada apenas por no máximo enumerável coordenadas. Em particular, se $f:[0,1]^{\kappa} \rightarrow \mathbb{R}$ é contínua, para $\kappa$ um cadinal de cofinalidade não enumerável, então existem $\alpha<\kappa$ e $g:[0,1]^{\alpha} \longrightarrow \mathbb{R}$ tais que $f=g \circ \pi_{\alpha}$, isto é, $f(x)=g(x \mid \alpha)$, para todo $x \in[0,1]^{\kappa}$.

O Teorema de Mibu também vale quando $f$ está definida em um compacto $K \subset[0,1]^{\kappa}$, para $\kappa$ de cofinalidade não enumerável, quando $K$ é o limite inverso de compactos $K_{\alpha} \subset[0,1]^{\alpha}$. De fato, estendendo $f$ continuamente a $[0,1]^{\kappa}$, usando o Teorema de Tietze, e tomando $\alpha$ e $\tilde{g}:[0,1]^{\alpha} \rightarrow \mathbb{R}$ como no Teorema de Mibu, para a extensão de $f$, temos que $f=g \circ \pi_{\alpha}$, para $g=\left.\tilde{g}\right|_{K_{\alpha}}$.

O próximo lema diz que, se uma sequência de funções contínuas definida em um limite inverso (sobre um cardinal de cofinalidade não enumerável) é separada por abertos, então, a partir de algum passo anterior, as funções definidas pelo Teorema de Mibu também são separadas por abertos e, em particular, têm suportes disjuntos.

Lema 5.19. Sejam $\lambda$ um cardinal de cofinalidade não enumerável, $K_{\alpha}$ um compacto, para cada $\alpha<\lambda$, e $K=\lim _{\leftarrow}\left(K_{\alpha}, \pi_{\alpha, \beta}, \lambda\right)$. Seja $\left(f_{n}\right)_{n \in \mathbb{N}}$ uma sequência de funções continuas de $K$ em $\mathbb{R}$ com suportes contidos em abertos disjuntos. Então existe $\gamma<\lambda$ tal que, se $\gamma<\alpha<\lambda$ e existe uma sequência de funções contínuas $g_{n}: K_{\alpha} \rightarrow \mathbb{R}$ tais que $g_{n}\left(\pi_{\alpha}(x)\right)=f_{n}(x)$, para todo $x \in K$ e $n \in \mathbb{N}$, então os suportes das funções $g_{n}$ estão contidos em abertos de $K_{\alpha}$ dois a dois disjuntos.

Demonstração: Seja $\left(U_{n}\right)_{n \in \mathbb{N}}$ uma sequência de abertos disjuntos em $K \operatorname{com} \operatorname{supp}\left(f_{n}\right) \subset U_{n}$. Pela compacidade de $K$, para cada $n \in \mathbb{N}$ existe uma união finita de abertos básicos contida em $U_{n}$ e que contém $\operatorname{supp}\left(f_{n}\right)$. Denotamos por $V_{n}$ a união finita de abertos básicos para cada $n \in \mathbb{N}$. Pela Proposição 1.3, para cada $n \in \mathbb{N}$ existe $\gamma_{n}$ tal que, para todo $\alpha>\gamma_{n}$, acontece

$$
\pi_{\alpha}^{-1}\left[\pi_{\alpha}\left[V_{n}\right]\right]=V_{n}
$$

e $\pi_{\alpha}\left[V_{n}\right]$ é aberto em $K_{\alpha}$.

Tome $\gamma=\sup \left\{\gamma_{n}: n \in \mathbb{N}\right\}$. Temos $\gamma<\lambda$, pela hipótese de $\lambda$ ter cofinalidade não enumerável. Portanto, para todo $\alpha>\gamma$ se verifica 5.15 e $\pi_{\alpha}\left[V_{n}\right]$ é aberto em $K_{\alpha}$, para todo $n \in \mathbb{N}$.

Por último, note que como $\operatorname{supp}\left(f_{n}\right)$ é compacto e as projeções $\pi_{\alpha}$ são contínuas, então

$$
\operatorname{supp}\left(g_{n}\right) \subset \pi_{\alpha}\left[\operatorname{supp}\left(f_{n}\right)\right] \subset \pi_{\alpha}\left[V_{n}\right]
$$

e, para $\alpha>\gamma$, os conjuntos $\pi_{\alpha}\left[V_{n}\right]$ são abertos e disjuntos em $K_{\alpha}$. 
Pelo Lema de König (ver [Jec03] ou [Kun80]), $2^{\omega}$ tem cofinalidade não enumerável. Nas construções que faremos nos próximos capítulos aplicaremos o Teorema 5.18 e o Teorema 5.19 para $\lambda=2^{\omega}$.

Lema 5.20 (Lema 6.1, [Faj12]). Um espaço compacto $K$ contém um subespaço homeomorfo a $\beta \mathbb{N}$ se, e somente se, existe uma sequência relativamente discreta $\left(x_{n}\right)_{n \in \mathbb{N}}$ em $K$ tal que, para todo $a \subset \mathbb{N}, \overline{\left\{x_{n}: n \in a\right\}} \cap \overline{\left\{x_{n}: n \in \mathbb{N} \backslash a\right\}}=\emptyset$.

O próximo lema teve o enunciado alterado, mas a validade do mesmo segue imediatamente da demonstração.

Lema 5.21 (Lema 3.9, [Faj12]). Seja $K$ um espaço compacto metrizável sem pontos isolados. Sejam $\left\{X_{n}: n \in \mathbb{N}\right\}$ e $\left\{Y_{n}: n \in \mathbb{N}\right\}$ famílias de subconjuntos de $K$ tais que $X_{n} \cap Y_{n}=\emptyset$ e $\overline{X_{n}} \cap \overline{Y_{n}} \neq \emptyset$. Seja $\left(x_{n}\right)_{n \in \mathbb{N}}$ uma sequência relativamente discreta em $K$ disjunta de $\bigcup_{m \in \mathbb{N}}\left(X_{n} \cup Y_{m}\right)$ e $b \subset \mathbb{N}$. Então existe uma sequência $\left(f_{n}\right)_{n \in b}$ de funções contínuas de $K$ em $[0,1]$, duas a duas disjuntas, tal que:

(i) $f_{n}\left(x_{n}\right)=1$, para todo $n \in b$, e $f_{n}\left(x_{m}\right)=0$, para $m \neq n, m \in \mathbb{N}$ e $n \in b$.

(ii) Para todo $x \in K \backslash D\left(\left(f_{n}\right)_{n \in b}\right), \pi^{-1}[\{x\}]=\{x\} \times[0,1]$, onde $\pi$ é a projeção canônica de $K\left(\left(f_{n}\right)_{n \in b}\right)$ em $K$.

(iii) Para todo $n \in \mathbb{N}, \overline{X_{n}^{\prime}} \cap \overline{Y_{n}^{\prime}} \neq \emptyset$ em $K\left(\left(f_{n}\right)_{n \in b}\right)$, onde $X_{n}^{\prime}=\left\{\left(x, \sum_{n \in b} f_{n}(x)\right): x \in X_{n}\right\}$ e $Y_{n}^{\prime}=\left\{\left(x, \sum_{n \in b} f_{n}(x)\right): x \in Y_{n}\right\}$.

(iv) $K\left(\left(f_{n}\right)_{n \in b}\right)$ é uma extensão completa de $K$. 


\section{Capítulo 6}

\section{Um espaço $C(K)$ com $2^{\omega}$ quocientes indecomponíveis não isomorfos}

Um espaço de Banach $X$ é dito de hereditariamente quociente indecomponível se nenhum quociente de dimensão infinita de $X$ é decomponível.

Não existem espaços de Banach da forma $C(K)$ que sejam hereditariamente quociente indecomponível. Com efeito, se $K$ é disperso, i.e., todo subespaço fechado contém pontos relativamente isolados (ver [Sem71], p. 147), então $K$ contém uma sequência (infinita) convergente (ver, por exemplo, [DT13]), o que implica que $C(K)$ contém $c_{0}$ complementado. Se $K$ não é disperso, então $C(K)$ contém um subespaço isomorfo a $\ell_{1}$ (ver [PS59]) e, portanto, pelo Teorema 4.1 em [Muj97], $C(K)$ contém um quociente isomorfo a $\ell_{2}$. Mas existem quocientes de $C(K)$ que sejam indecomponíveis? Mais ainda, podemos construir $C(K)$ com muitos quocientes indecomponíveis?

Lembramos que subespaços fechados de $K$ induzem quocientes de $C(K)$, através da transformação linear da restrição. Koszmider, no final de [Kos04], comenta sobre a possibilidade de construir um espaço compacto $K$ tal que para todo $L \subset K$ fechado, $C(L)$ tem poucos operadores. Como foi mencionado na introdução, o primeiro resultado nesta linha foi apresentado por Fajardo em [Faj12] e assume o axioma $\diamond$. Na construção de $K$ conexo, com essa propriedade, obtemos vários subespaços $L$ de $K$ tais que $C(L)$ é indecomponível. Especificamente, conforme provado no Corolário 5.4 de [Faj12], assumindo $\diamond$ existe $C(K)$ contendo $2^{\omega}$ quocientes indecomponíveis da forma $C(L)$ não isomorfos. Segue também dessa propriedade que $K$, como em [Faj12], não contém $\beta \mathbb{N}$ como subespaço, visto que $C(\beta \mathbb{N})=l_{\infty}$ tem muitos operadores não multiplicadores fracos. No mesmo artigo é provado, assumindo $\mathrm{CH}$, que existe um espaço de Koszmider contendo cópia de $\beta \mathbb{N}$.

Neste capítulo mostraremos que, assumindo $\diamond$, existe $K$ um espaço de Koszmider conexo contendo cópia homeomórfica de $\beta \mathbb{N}$ e uma quantidade não enumerável de subespaços conexos e de Koszmider. Em particular, $C(K)$ contém $2^{\omega}$ quocientes indecomponíveis e também contém $l_{\infty}$ como quociente.

A construção utiliza indução transfinita, como aquela feita em [Kos04], mas trocando as extensões fortes, usadas em [Kos04], por extensões completas, que foram usadas em [Faj12]. 


\subsection{Construção do espaço}

A construção é por indução transfinita e segue as ideias de Koszmider, em [Kos04], e Fajardo, em [Faj12]. O espaço $K$ é o limite de um sistema inverso $\lim _{\leftarrow}\left(K_{\alpha}, \pi_{\alpha, \beta}, \omega_{1}\right)$, onde cada $K_{\alpha}$ é um subespaço compacto de $[0,1]^{\alpha}$. No caso de um ordinal sucessor $\alpha+1$, o espaço $K_{\alpha+1}$ é obtido como uma extensão completa do espaço $K_{\alpha}$, por sequências de funções contínuas com suportes contidos em abertos disjuntos.

A construção nos passos sucessores se alterna entre "destruir" um par de abertos-borboleta, usando o Lema 5.12, em separar uma sequência relativamente discreta, usando o Lema 5.21 (para garantir que $\beta \mathbb{N}$ está em $K$ ), e "destruir" um operador não multiplicador fraco, através do Lema 5.16 .

O espaço $C(K)$ contém $2^{\omega}$ quocientes indecomponíveis e esses quocientes são os espaços das funções contínuas sobre fechos de subconjuntos abertos de $K$. Além disso, $K$ contém uma cópia homeomórfica de $\beta \mathbb{N}$, de onde segue que $C(K)$ possui $l_{\infty}$ como quociente.

Teorema 6.1. $(Z F C+\diamond)$ Existe um espaço compacto $K \subset[0,1]^{2^{\omega}}$ tal que:

(a) $K_{I}$ é infinito e conexo, para todo intervalo não degenerado $I \subset[0,1]$;

(b) $K$ não contém abertos borboleta e, mais ainda, $\overline{K_{[0, r[}}$ não contém abertos-borboleta, para todo $0<r \leq 1$

(c) Se $M \subset[0,1]$ é raro, então $\pi^{-1}[M]$ é raro, em $K$;

(d) $K$ é um espaço de Koszmider e, para todo $0<r \leq 1, \overline{K_{[0, r[}}$ é um espaço de Koszmider;

(e) $K$ contém um subespaço homeomorfo a $\beta \mathbb{N}$.

Demonstração: Por indução transfinita, construímos compactos $K_{\alpha} \subset[0,1] \times[0,1]^{\alpha}$, para $1 \leq$ $\alpha \leq 2^{\omega}, Q_{\alpha}$ subconjunto denso em $K_{\alpha}$ e sequências $\left\{p_{n}^{\alpha}: n \in \mathbb{N}\right\}$ em $K_{\alpha}$, para $1 \leq \alpha \leq 2^{\omega}$. Tomamos $K_{1}=[0,1] \times[0,1], Q_{1}=\mathbb{Q}^{2} \cap[0,1]^{2}$ e $\left\{p_{n}^{1}: n \in \mathbb{N}\right\}$ uma sequência em $[0,1] \times[0,1]$ relativamente discreta e disjunta de $Q_{1}$.

Usando o axioma $\diamond$, fixamos uma enumeração

$$
\left(\left(l_{n}(\alpha)_{n \in \mathbb{N}}\right),\left(f_{n}(\alpha)\right)_{n \in \mathbb{N}}, U(\alpha), V(\alpha), \varepsilon(\alpha), N(\alpha),\left(\mu_{n}(\alpha)\right)_{n \in \mathbb{N}}, i(\alpha)\right)_{\alpha<2^{\omega}}
$$

com:

A.1. $\left(l_{n}(\alpha)\right)_{n \in \mathbb{N}}$ uma sequência crescente de inteiros positivos;

A.2. $f_{n}(\alpha):[0,1]^{\omega_{1}} \rightarrow[0,1]$ funções contínuas;

A.3. $U(\alpha)$ e $V(\alpha)$ uniões enumeráveis de abertos básicos em $[0,1]^{\omega_{1}}$;

A.4. $\varepsilon(\alpha)>0$;

A.5. $N(\alpha) \subset \mathbb{N}$;

A.6. $\left(\mu_{n}(\alpha)\right)_{n \in \mathbb{N}}$ sequência limitada de funções $\mu_{n}(\alpha): \mathcal{B}_{\alpha} \rightarrow \mathbb{R}$ que representam medidas de Radon em $[0,1]^{\alpha}$; 
A.7. $i(\alpha) \in 3$;

tal que, dados $\beta<\omega_{1}$ e

B.1. $\left(l_{n}\right)_{n \in \mathbb{N}}$ uma sequência crescente de inteiros positivos;

B.2. $\left(f_{n}\right)_{n \in \mathbb{N}}$ uma sequência de funções contínuas de $[0,1]^{\omega_{1}}$ em $[0,1]$;

B.3. $U$ e $V$ uniões enumeráveis de abertos básicos em $[0,1]^{\omega_{1}}$;

B.4. Um número real $\varepsilon>0$;

B.5. $N \subset \mathbb{N}$;

B.6. $\left(\mu_{n}\right)_{n \in \mathbb{N}}$ uma sequência de funções de $\mathcal{B}_{\omega_{1}}$ em $\mathbb{R}$ que representam medidas de Radon;

B.7. $i \in 3$;

existe $\alpha$ tal que $\beta<\alpha<\omega_{1}$ e

C.1. $l_{n}=l_{n}(\alpha)$, para todo $n \in \mathbb{N}$;

C.2. $f_{n}=f_{n}(\alpha)$, para todo $n \in \mathbb{N}$;

C.3. $U(\alpha)=U$ e $V(\alpha)=V$;

C.4. $\varepsilon(\alpha)=\varepsilon ;$

C.5. $N(\alpha)=N$;

C.6. $\mu_{n}(\alpha)=\left.\mu_{n}\right|_{\mathcal{B}_{\alpha}}$, ou seja $\mu_{n} \circ \pi_{\alpha}^{-1}(B)=\mu_{n}(\alpha)(B)$ para todo $B \in \mathcal{B}_{\alpha}$;

C.7. $i(\alpha)=i$.

Esta enumeração é possível pelo Lema 1.23 e porque a separabilidade de $[0,1]^{2^{\omega}}$ implica que existem $2^{\omega}$ funções contínuas em $[0,1]^{\omega_{1}}$ e, como $[0,1]$ tem base enumerável e existem $2^{\omega}$ subconjuntos finitos de $2^{\omega}=\omega \times 2^{\omega}$, existem $2^{\omega}$ pares de abertos que são uniões enumeráveis de abertos elementares.

Em um passo $\alpha<\omega_{1}$ supomos que temos construídos $K_{\alpha},\left(a_{\beta}^{i}\right)_{\beta<\alpha, i \in 2},\left(b_{\beta}^{i}\right)_{\beta<\alpha, i \in 2}, Q_{\alpha}=\left\{q_{n}^{\alpha}\right.$ : $n \in \mathbb{N}\}$ e uma sequência $\left\{p_{n}^{\alpha}: n \in \mathbb{N}\right\}$ em $K_{\alpha}$, tais que

D.1. $K_{\alpha} \subset[0,1]^{\alpha}$ é compacto e conexo;

D.2. $b_{\beta}^{i}$ e $a_{\beta}^{i} \backslash b_{\beta}^{i}$ são infinitos e tais que $b_{\beta}^{i} \subset a_{\beta}^{i} \subset \mathbb{N}$, para todos $i \in 2$ e $\beta<\alpha$;

D.3. $Q_{\alpha}$ é denso em $K_{\alpha}$ e $\left.q_{n}^{\alpha}\right|_{\beta}=q_{n}^{\beta}$, se $\beta<\alpha$;

D.4. $\overline{\left\{q_{n}^{\alpha}: n \in b_{\beta}^{i}\right\}} \cap \overline{\left\{q_{n}^{\alpha}: n \in a_{\beta}^{i} \backslash b_{\beta}^{i}\right\}} \neq \emptyset$, para todos $i \in 2$ e $\beta<\alpha$;

D.5. $\left\{p_{n}^{\alpha}: n \in \mathbb{N}\right\}$ é relativamente discreta e $\left.p_{n}^{\alpha}\right|_{\beta}=p_{n}^{\beta}$, para todo $\beta<\alpha$ e $n \in \mathbb{N}$. 
Se $\alpha^{\prime}$ é ordinal limite, definimos $K_{\alpha^{\prime}}$ como o limite do sistema inverso $\left(K_{\alpha}, \pi_{\alpha, \beta}, \alpha^{\prime}\right)$, onde $\pi_{\alpha, \beta}: K_{\beta} \rightarrow K_{\alpha}$ é definido por $\pi_{\alpha, \beta}\left(x^{\beta}\right)=\left.x^{\beta}\right|_{\alpha}$, para $\alpha \leq \beta<\alpha^{\prime}$, e $Q_{\alpha}:=\left\{q_{n}^{\alpha^{\prime}} \in K_{\alpha^{\prime}}:\left.q_{n}^{\alpha^{\prime}}\right|_{\beta}=\right.$ $\left.q_{n}^{\beta} \in Q_{\beta}, \beta<\alpha^{\prime}, n \in \mathbb{N}\right\}$. Note que os conjuntos $a_{\beta}^{i}$ e $b_{\beta}^{i}$ já estão definidos para todo $\beta<\alpha^{\prime}$ e $i \in 2$, de modo que a condição D.2 já está satisfeita. Como limite inverso preserva compacidade e conexidade (veja [Eng89]), vale D.1.

Provemos D.3, isto é, que $Q_{\alpha^{\prime}}$ é denso em $K_{\alpha^{\prime}}$ (a segunda parte de D.3 é trivial da construção). Se $V$ é um aberto elementar de $K_{\alpha^{\prime}}$, existe $\alpha<\alpha^{\prime}$ tal que $\pi_{\alpha, \alpha^{\prime}}[V]$ é aberto em $K_{\alpha}$ e $\pi_{\alpha, \alpha^{\prime}}^{-1}\left[\pi_{\alpha, \alpha^{\prime}}[V]\right]=V$ (veja a Proposição 1.3). Pela densidade de $Q_{\alpha}$ em $K_{\alpha}$, temos $Q_{\alpha} \cap \pi_{\alpha, \alpha^{\prime}}[V] \neq \emptyset$ e, portanto, $Q_{\alpha^{\prime}} \cap V \neq \emptyset$.

A prova de D.4 é a mesma que do Lema 5.17. Para garantir D.5, definimos para cada $n \in \mathbb{N}$ o $p_{n}^{\alpha} \in K_{\alpha}$ como tal que $\left.p_{n}^{\alpha}\right|_{\beta}=p_{n}^{\beta}$ para todo $\beta<\alpha$.

Trataremos agora do passo sucessor. Construído o compacto $K_{\alpha}$, diremos que o ordinal $\alpha$ é um passo não trivial se:

$$
i(\alpha)=0 \mathrm{e}
$$

E.1. $\left\{q_{l_{n}(\alpha)}^{\alpha}: n \in \mathbb{N}\right\}$ é relativamente discreto em $K_{\alpha}$;

E.2. existem funções $g_{n}: K_{\alpha} \rightarrow[0,1]$ separadas por abertos disjuntos e tais que $f_{n}(\alpha)(y)=g_{n}(x)$, sempre que $\left.y\right|_{\alpha}=x$, para todo $x \in K_{\alpha}$;

E.3. $g_{n}\left(q_{l_{m}(\alpha)}^{\alpha}\right)=0$ para todo $m, n \in \mathbb{N}$

E.4. $\left|\int_{K_{\alpha}} g_{n} d \mu_{n}(\alpha)\right|>\varepsilon(\alpha)$, para todo $n \in \mathbb{N}$;

ou $i(\alpha)=1 \mathrm{e}$

F.1. $\pi_{\alpha}^{-1}\left[\pi_{\alpha}[U(\alpha)]\right]=U(\alpha)$ e $\pi_{\alpha}^{-1}\left[\pi_{\alpha}[V(\alpha)]\right]=V(\alpha)$, onde $\pi_{\alpha}:[0,1]^{\omega_{1}} \rightarrow[0,1]^{\alpha}$ é a projeção canônica;

F.2. $\overline{\pi_{\alpha}[U(\alpha)] \cap K_{\alpha}} \cap \overline{\pi_{\alpha}[V(\alpha)] \cap K_{\alpha}} \neq \emptyset$ com o fecho tomado em $K_{\alpha}$;

ou $i(\alpha)=2$.

Se $K_{\alpha}$ não verifica as condições acima, então dizemos que $\alpha$ é um passo trivial e definimos $K_{\alpha+1}:=K_{\alpha} \times\{0\}$ e $Q_{\alpha+1}:=\left\{\left(q_{n}^{\alpha}\right) \frown 0: n \in \mathbb{N}\right\}$. Os conjuntos $a_{\alpha}$ e $b_{\alpha}$ podem ser escolhidos como $a_{\beta}$ e $b_{\beta}$ para algum $\beta<\alpha$. A sequência $\left\{p_{n}^{\alpha+1}: n \in \mathbb{N}\right\}$ é escolhida tomando $p_{n}^{\alpha+1}=\left(p_{n}^{\alpha}\right)^{\frown} 0$ para cada $n \in \mathbb{N}$.

Se $\alpha$ é um passo não trivial e $i(\alpha)=1$, seja $x \in \overline{\pi_{\alpha}[U(\alpha)] \cap K_{\alpha}} \cap \overline{\pi_{\alpha}[V(\alpha)] \cap K_{\alpha}}$. Aplicando o Lema 5.12 para $K=K_{\alpha}, Q=Q_{\alpha}, \mathcal{P}=\left\{\left(b_{\beta}^{i}, a_{\beta}^{i} \backslash b_{\beta}^{i}\right): \beta<\alpha, i \in 2\right\}, h$ uma função qualquer (uma constante, por exemplo), e os abertos (ver Proposição 1.2 do Capítulo 1) com a intersecção dos fechos não vazia como sendo $\pi_{\alpha}[U(\alpha)] \cap K_{\alpha}$ e $\pi_{\alpha}[V(\alpha)] \cap K_{\alpha}$, obtemos $b \subset \mathbb{N}$ infinito, sequências estritamente crescentes de naturais $\left(l_{n}^{i}\right)_{n \in \mathbb{N}} \operatorname{com} i \in\{0,1\}$, sequências de abertos $U_{n}$ e $V_{n}$ e uma sequência $\left(f_{n}\right)_{n \in \mathbb{N}}$ de funções duas a duas disjuntas que verificam (d) até (l) do Lema 5.12. Trocando $b$ por um subconjunto, se for necessário, assumimos que $b$ é infinito e co-infinito. 
Definimos $K_{\alpha+1}=K_{\alpha}\left(\left(f_{n}\right)_{n \in b}\right), Q_{\alpha+1}=\left\{q_{n}^{\alpha \frown t_{n}}: n \in \mathbb{N}\right\}$ onde $t_{n}=\sum_{m \in b} f_{m}\left(q_{n}^{\alpha}\right)$, se $q_{n}^{\alpha} \in D\left(\left(f_{n}\right)_{n \in b}\right)$, e $t_{n}=0$ caso contrário, $a_{\alpha}^{1}=\left\{l_{n}^{i}: n \in b, i \in 2\right\}, b_{\alpha}^{1}:=\left\{l_{n}^{1}: n \in b\right\}, a_{\alpha}^{0}=\left\{l_{n}^{i}: n \in\right.$ $\mathbb{N} \backslash b, i \in 2\}, b_{\alpha}^{0}:=\left\{l_{n}^{1}: n \in \mathbb{N} \backslash b\right\}$. Note que, em particular, por (k) do Lema 5.12, temos

$$
(x, i) \in \overline{\left\{q_{n}^{\alpha \frown} i: n \in b_{\alpha}^{i}\right\}} \cap \overline{\left\{q_{n}^{\alpha \frown} i: n \in a_{\alpha}^{i} \backslash \in b_{\alpha}^{i}\right\}}
$$

para $i \in\{0,1\}$ e com o fecho sendo tomado em $K_{\alpha+1}$ e

$$
(x, 0),(x, 1) \in \overline{\pi_{\alpha, \alpha+1}^{-1}\left[\pi_{\alpha}[U(\alpha)] \cap K_{\alpha}\right]} \cap \overline{\pi_{\alpha, \alpha+1}^{-1}\left[\pi_{\alpha}[V(\alpha)] \cap K_{\alpha}\right]} .
$$

A sequência $\left\{p_{n}^{\alpha+1}: n \in \mathbb{N}\right\}$ é definida tomando $p_{n}^{\alpha+1}$ como sendo um ponto arbitrário em $\pi_{\alpha, \alpha+1}^{-1}\left[\left\{p_{n}^{\alpha}\right\}\right]$.

Faremos agora a construção no caso não trivial e $i(\alpha)=0$.

Por $C H$ (que segue de $\diamond$ ), temos $|\alpha| \leq \omega$ e, portanto, $K_{\alpha}$ é metrizável. Aplicando o Lema 5.16 para $K_{\alpha}, Q_{\alpha}, \varepsilon(\alpha),\left(\mu_{n}(\alpha)\right)_{n \in \mathbb{N}},\left(g_{n}\right)_{n \in \mathbb{N}},\left(l_{n}\right)_{n \in \mathbb{N}}$ e $\mathcal{P}_{\alpha}=\left\{\left(b_{\beta}^{i}, a_{\beta}^{i} \backslash b_{\beta}^{i}\right): i \in\{0,1\}, \beta<\alpha\right\}$, existem $\delta>0$, funções contínuas $g_{n}^{\prime}: K_{\alpha} \rightarrow[0,1]$ e subconjuntos infinitos $a, b^{\prime}$ de $\mathbb{N}$ tais que $b^{\prime} \subset a$ e, para todo $b \subset b^{\prime}$, temos:

- $\operatorname{supp}\left(g_{n}^{\prime}\right) \subset \operatorname{supp}\left(g_{n}\right)$ para todo $n \in \mathbb{N}$;

- $\left|\int g_{n}^{\prime} d \mu_{n}(\alpha)\right|>\delta$ e $\Sigma\left\{\int g_{m}^{\prime} d\left|\mu_{n}(\alpha)\right|: m \neq n, m \in a\right\}<\delta / 3$, para todo $n \in a$;

- $K_{\alpha}\left(\left(g_{n}^{\prime}\right)_{n \in b}\right)$ é uma extensão completa;

- $\overline{\left\{\left(q_{l_{n}}^{\alpha}\right)^{\prime}: n \in b\right\}} \cap \overline{\left\{\left(q_{l_{n}}^{\alpha}\right)^{\prime}: n \in a \backslash b\right\}} \neq \emptyset$, onde $q_{m}^{\prime}$ é definido como $\left(q_{m}, \sum_{n \in b} g_{n}^{\prime}\left(q_{m}\right)\right)$;

- $\overline{\left\{\left(q_{n}^{\alpha}\right)^{\prime}: n \in b_{\beta}^{i}\right\}} \cap \overline{\left\{\left(q_{n}^{\alpha}\right)^{\prime}: n \in a_{\beta}^{i} \backslash b_{\beta}^{i}\right\}} \neq \emptyset$, para todo $\beta<\alpha$ e $i \in\{0,1\}$.

Fixamos $b \subset b^{\prime}$ tal que $a \backslash b$ é infinito e definimos $K_{\alpha+1}:=K_{\alpha}\left(\left(g_{n}^{\prime}\right)_{n \in b}\right), a_{\alpha}^{i}:=a$ e $b_{\alpha}^{i}:=b$, para $i \in\{0,1\}, Q_{\alpha+1}=\left\{q_{n}^{\alpha \smile t}: n \in \mathbb{N}\right\}$ com $t=\sum_{m \in b} g_{m}^{\prime}\left(q_{n}^{\alpha}\right)$. De novo, a sequência $\left\{p_{n}^{\alpha+1}: n \in \mathbb{N}\right\}$ é definida tomando $p_{n}^{\alpha+1}$ como sendo um ponto arbitrário em $\pi_{\alpha, \alpha+1}^{-1}\left[\left\{p_{n}^{\alpha}\right\}\right]$.

Agora tratemos de construir $K_{\alpha+1}$ quando $i(\alpha)=2$.

Como $\left\{p_{n}^{\alpha}: n \in \mathbb{N}\right\}$ é disjunto de $Q_{\alpha}$, aplicamos o Lema 5.21 para $K=K_{\alpha},\left\{\left(X_{n}, Y_{n}\right): n \in \mathbb{N}\right\}$ como sendo $\left\{\left(\left\{q_{n}^{\alpha}: n \in b_{\beta}^{i}\right\},\left\{q_{n}^{\alpha}: n \in a_{\beta}^{i} \backslash b_{\beta}^{i}\right\}\right): \beta<\alpha, i \in 2\right\}$, a sequência $\left(q_{n}^{\alpha}\right)_{n \in \mathbb{N}}$ e o subconjunto $N(\alpha)$ de $\mathbb{N}$. Assim, existe uma sequência $\left(f_{n}\right)_{n \in N(\alpha)}$ de funções contínuas de $K_{\alpha}$ em [0,1], tais que

G.1. $f_{n}\left(p_{n}^{\alpha}\right)=1$, para todo $n \in N(\alpha)$, e $f_{n}\left(p_{m}^{\alpha}\right)=0$, se $m \neq n, m \in \mathbb{N}$ e $n \in N(\alpha)$;

G.2. para cada $x \in K_{\alpha} \backslash D\left(\left(f_{n}\right)_{n \in N(\alpha)}\right),\{x\} \times[0,1] \subset K_{\alpha}\left(\left(f_{n}\right)_{n \in N(\alpha)}\right)$;

G.3. para todo $\beta<\alpha$ e $i \in 2$,

$$
\overline{\left\{\left(q_{n}^{\alpha}\right)^{\prime}: n \in b_{\beta}^{i}\right\}} \cap \overline{\left\{\left(q_{n}^{\alpha}\right)^{\prime}: n \in a_{\beta}^{i} \backslash b_{\beta}^{i}\right\}} \neq \emptyset
$$

com o fecho sendo tomado em $K_{\alpha}\left(\left(f_{n}\right)_{n \in N(\alpha)}\right)$, e onde $\left(q_{n}^{\alpha}\right)^{\prime}=\left(q_{n}^{\alpha}, \sum_{n \in N(\alpha)} f_{n}\left(q_{n}^{\alpha}\right)\right)$ se $q_{n}^{\alpha} \in$ $D\left(\left(f_{n}\right)_{n \in N(\alpha)}\right)$ e $\left(q_{n}^{\alpha}\right)^{\prime}=\left(q_{n}^{\alpha}, 0\right)$ caso contrário;

G.4. $K_{\alpha}\left(\left(f_{n}\right)_{n \in N(\alpha)}\right)$ é uma extensão completa de $K$. 
Isso completa a construção no caso ordinal sucessor.

Agora definimos $K=K_{\omega_{1}}, Q=Q_{\omega_{1}}$ e demonstramos que verificam o teorema.

Para provar (a), seja $I \subset[0,1]$ não degenerado. Suponhamos, primeiro que $I$ é fechado. Então $\pi_{2}\left[K_{I}\right]=I \times[0,1]$ e, portanto, é compacto e conexo. O Lema 5.6 implica que $\pi_{2, \omega_{1}}^{-1}[I \times[0,1]]=K_{I}$ é compacto e conexo.

Sejam $0 \leq a<b \leq 1$. Se $I=] a, b\left[\right.$, então $K_{I}=\bigcup_{n=n_{0}}^{\infty} K_{\left[a+\frac{1}{n}, b-\frac{1}{n}\right]}$, onde $n_{0}$ é escolhido tal que $a+\frac{1}{n_{0}}<b-\frac{1}{n_{0}}$. Como $K_{\left[a+\frac{1}{n_{0}}, b-\frac{1}{n_{0}}\right]}$ intersecta todos os $K_{\left[a+\frac{1}{n}, b-\frac{1}{n}\right]}$, temos que $K_{I}$ é conexo.

Se $I=\left[a, b\left[\right.\right.$, então $K_{I}=\bigcup_{n=n_{0}}^{\infty} K_{\left[a, b-\frac{1}{n}\right]}$, com $n_{0}$ tal que $a<b-\frac{1}{n_{0}}$, e, portanto, $K_{I}$ é conexo. O caso $I=] a, b]$ é análogo, concluindo a prova de (a).

Para demonstrar (b), como $\overline{K_{[0, r}}$ é o fecho de um aberto, pelo Lema 2.10 é suficiente demonstrar que $K$ não contém abertos-borboletas. Para isso provamos que, se dois abertos disjuntos em $K$ têm a intersecção de seus fechos não vazia, então essa deve conter pelo menos dois pontos. Para facilitar a notação, nesta parte da prova entenderemos que $\pi_{\alpha}$ é a projeção canônica de $[0,1]^{\omega_{1}}$ sobre $[0,1]^{\alpha}$ ou de $K$ sobre $K_{\alpha}$, dependendo do contexto.

Sejam $U$ e $V$ abertos disjuntos em $K$ e $z \in \bar{U} \cap \bar{V}$. Como $K$ é separável, o fecho de qualquer aberto em $K$ é igual ao fecho de uma união enumerável de abertos básicos da topologia de $K$. Assim, existem sequências $\left\{\pi_{\alpha_{n}}^{-1}\left[B_{\alpha_{n}} \cap K_{\alpha_{n}}\right]\right\}_{n \in \mathbb{N}}$ e $\left\{\pi_{\alpha_{n}^{\prime}}^{-1}\left[B_{\alpha_{n}^{\prime}} \cap K_{\alpha_{n}^{\prime}}\right]\right\}_{n \in \mathbb{N}}$ de abertos básicos da topologia de $K$, onde $B_{\alpha_{n}}$ e $B_{\alpha_{n}^{\prime}}$ são abertos em $[0,1]^{\alpha_{n}}$ e $[0,1]^{\alpha_{n}^{\prime}}$, respectivamente, e $\bar{U}=\overline{\bigcup_{n \in \mathbb{N}} \pi_{\alpha_{n}}^{-1}\left[B_{\alpha_{n}} \cap K_{\alpha_{n}}\right]}$ e $\bar{V}=\overline{\bigcup_{n \in \mathbb{N}} \pi_{\alpha_{n}^{\prime}}^{-1}\left[B_{\alpha_{n}^{\prime}} \cap K_{\alpha_{n}^{\prime}}\right]}$, com o fecho sendo tomado em $K$.

Sejam

$$
\tilde{U}:=\bigcup_{n \in \mathbb{N}} \pi_{\alpha_{n}}^{-1}\left[B_{\alpha_{n}}\right] \text { e } \tilde{V}:=\bigcup_{n \in \mathbb{N}} \pi_{\alpha_{n}^{\prime}}^{-1}\left[B_{\alpha_{n}^{\prime}}\right],
$$

com $\tilde{U} \cap K \subset U$ e $\tilde{V} \cap K \subset V$. Pela enumeração dada no começo, existem cofinais $\alpha$ 's em $2^{\omega}$ tais que $U(\alpha)=\tilde{U}$ e $V(\alpha)=\tilde{V}$ e $i(\alpha)=1$. Tome $\gamma=\sup \left\{\alpha_{n}, \alpha_{n}^{\prime}: n \in \mathbb{N}\right\}<2^{\omega}$.

Vejamos que $\pi_{\alpha}(z) \in \overline{\pi_{\alpha}[\tilde{U}] \cap K_{\alpha}} \cap \overline{\pi_{\alpha}[\tilde{V}] \cap K_{\alpha}}$. Seja $O_{z}$ um aberto em $K_{\alpha}$ contendo $\pi_{\alpha}(z)$. Como $z \in \overline{\tilde{U} \cap K}$, então $\pi_{\alpha}^{-1}\left[O_{z}\right] \cap \tilde{U} \cap K \neq \emptyset$ e, portanto, $O_{z} \cap \pi_{\alpha}[\tilde{U}] \cap K_{\alpha} \neq \emptyset$. Assim, $\pi_{\alpha}(z) \in \overline{\pi_{\alpha}[\tilde{U}] \cap K_{\alpha}}$. Analogamente, $\pi_{\alpha}(z) \in \overline{\pi_{\alpha}[\tilde{V}] \cap K_{\alpha}}$, verificando a condição F.2. do passo não trivial.

Mostraremos agora a condição F.1. Seja $\alpha \geq \gamma$. Se $x, y \in[0,1]^{\omega_{1}}$ são tais que $\left.x\right|_{\alpha}=\left.y\right|_{\alpha}$, então $x \in \pi_{\alpha_{n}}^{-1}\left[B_{\alpha_{n}}\right]$ se, e somente se, $y \in \pi_{\alpha_{n}}^{-1}\left[B_{\alpha_{n}}\right]$. Logo, $y \in \pi_{\alpha}^{-1}\left[\pi_{\alpha}\left[\pi_{\alpha_{n}}^{-1}\left[B_{\alpha_{n}}\right]\right]\right]$ implica $y \in \pi_{\alpha_{n}}^{-1}\left[B_{\alpha_{n}}\right]$ e, portanto, $\pi_{\alpha}^{-1}\left[\pi_{\alpha}\left[\pi_{\alpha}^{-1}\left[B_{\alpha_{n}}\right]\right]\right]=\pi_{\alpha_{n}}^{-1}\left[B_{\alpha_{n}}\right]$ para todo $n \in \mathbb{N}$. O mesmo se aplica a $\alpha_{n}^{\prime}$, de onde concluímos que

$$
\pi_{\alpha}^{-1}\left[\pi_{\alpha}[\tilde{U}]\right]=\tilde{U}, \quad \pi_{\alpha}^{-1}\left[\pi_{\alpha}[\tilde{V}]\right]=\tilde{V}
$$

verificando a condição F.1 do passo não trivial.

Pela construção, existem $x \in K_{\alpha}$ e sequências $a_{\alpha}^{i}$ e $b_{\alpha}^{i}$ de inteiros, com $i \in 2$, tais que

$$
(x, i) \in \overline{\left\{\left.q_{n}\right|_{\alpha+1}: n \in b_{\alpha}^{i}\right\}} \cap \overline{\left\{\left.q_{n}\right|_{\alpha+1}: n \in a_{\alpha}^{i} \backslash b_{\alpha}^{i}\right\}}
$$

com os fechos sendo tomados em $K_{\alpha+1}$ e de modo que $\left.q_{n}\right|_{\alpha+1} \in \pi_{\alpha, \alpha+1}^{-1}\left[\pi_{\alpha}[\tilde{U}] \cap K_{\alpha}\right]$, se $n \in b_{\alpha}^{i}$, e $\left.q_{n}\right|_{\alpha+1} \in \pi_{\alpha, \alpha+1}^{-1}\left[\pi_{\alpha}[\tilde{V}] \cap K_{\alpha}\right]$, se $n \in a_{\alpha}^{i} \backslash b_{\alpha}^{i}$. Mas, por D.4, temos que, para $i \in 2$, a intersecção dos 
fechos em $K_{\alpha^{\prime}}$ de $\left\{\left.q_{n}\right|_{\alpha^{\prime}}: n \in b_{\alpha}^{i}\right\}$ e $\left\{\left.q_{n}\right|_{\alpha^{\prime}}: n \in a_{\alpha}^{i} \backslash b_{\alpha}^{i}\right\}$ é não vazia, para todo $\alpha^{\prime} \geq \alpha+1>\gamma$. Portanto, pelo Lema 5.17

$$
\overline{\left\{q_{n}: n \in b_{\alpha}^{i}\right\}} \cap \overline{\left\{q_{n}: n \in a_{\alpha}^{i} \backslash b_{\alpha}^{i}\right\}} \neq \emptyset,
$$

para $i \in 2$ e com o fecho como sendo tomado em $K$.

Agora vejamos que para cada $i \in\{0,1\}$ a intersecção em (6.3) contém um ponto $z_{i}$ e que $z_{0} \neq z_{1}$. Note que, para cada $i \in\{0,1\}$, os conjuntos $\left(K_{\alpha} \times\left[0, \frac{1}{3}\right]\right) \cap K_{\alpha+1}$ e $\left(K_{\alpha} \times\left[\frac{2}{3}, 1\right]\right) \cap K_{\alpha+1}$ são fechados disjuntos em $K_{\alpha+1}$ e tais que

$$
\left\{\left.q_{n}\right|_{\alpha+1}: n \in a_{\alpha}^{0}\right\} \subset K_{\alpha} \times[0,1 / 3], \text { e }\left\{\left.q_{n}\right|_{\alpha+1}: n \in a_{\alpha}^{1}\right\} \subset K_{\alpha} \times[2 / 3,1] .
$$

Como $\pi_{\alpha+1}$ é contínua e as imagens inversas de conjuntos disjuntos são disjuntas, então por (6.3) existem $z_{0} \in \pi_{\alpha+1}^{-1}\left[K_{\alpha} \times[0,1 / 3]\right]$ e $z_{1} \in \pi_{\alpha+1}^{-1}\left[K_{\alpha} \times[2 / 3,1]\right]$, e $z_{i} \in \overline{\left\{q_{n}: n \in b_{\alpha}^{i}\right\}} \cap \overline{\left\{q_{n}: n \in a_{\alpha}^{i} \backslash b_{\alpha}^{i}\right\}}$.

Finalmente, observe que, se $q_{n} \in Q,\left.\operatorname{com} q_{n}\right|_{\alpha} \in \pi_{\alpha}[\tilde{U}] \cap K_{\alpha}$ e $q_{n} \notin K \cap \tilde{U}$, então $\pi_{\alpha}^{-1}\left[\pi_{\alpha}[\tilde{U}]\right] \neq \tilde{U}$ contradizendo (6.1). Portanto, $\left.q_{n}\right|_{\alpha} \in \pi_{\alpha}[\tilde{U} \cap K]$ para todo $n \in b_{\alpha}^{i}$. Analogamente, $\left.q_{n}\right|_{\alpha} ^{i} \in \pi_{\alpha}[\tilde{V} \cap K]$, para todo $n \in a_{\alpha}^{i} \backslash b_{\alpha}^{i}$ e $i \in 2$. Além disso, como $\tilde{U} \cap K=\bigcup_{n \in \mathbb{N}} \pi_{\alpha_{n}}^{-1}\left[B_{\alpha_{n}} \cap K_{\alpha_{n}}\right]$ e $\tilde{V} \cap K=$ $\bigcup_{n \in \mathbb{N}} \pi_{\alpha_{n}^{\prime}}^{-1}\left[B_{\alpha_{n}^{\prime}} \cap K_{\alpha_{n}^{\prime}}\right]$, então

$$
\pi_{\alpha}^{-1}\left[\pi_{\alpha}[\tilde{U} \cap K]\right]=\tilde{U} \cap K, \text { e } \pi_{\alpha}^{-1}\left[\pi_{\alpha}[\tilde{V} \cap K]\right]=\tilde{V} \cap K,
$$

para todo $\alpha>\gamma$. Assim, $\left\{q_{n}: n \in b_{\alpha}^{i}\right\} \subset \tilde{U} \cap K$ e $\left\{q_{n}: n \in a_{\alpha}^{i} \backslash b_{\alpha}^{i}\right\} \subset \tilde{V} \cap K$. Portanto $z_{0}, z_{1} \in \overline{\tilde{U} \cap K} \cap \overline{\tilde{V} \cap K}=\bar{U} \cap \bar{V}$, concluindo a prova de (b).

Para provar (c), primeiro notemos que, se $M \subset[0,1]$ é raro, então $M \times[0,1]$ é raro em $[0,1]^{2}$. De fato, se $U \times V$ é um aberto básico de $[0,1]^{2}$, por $M$ ser raro existe $U^{\prime} \subset U$ aberto não vazio disjunto de $M$. Logo, $U^{\prime} \times V$ é um subconjunto aberto de $U \times V$ disjunto de $M \times[0,1]$, provando que $M \times[0,1]$ é raro em $[0,1]^{2}$. Do Lema 5.8 segue, portanto, o item (c).

Agora demonstraremos que $K$ verifica (d). Pelo item (b) e pelos Lemas 2.7 e 2.9, para provar que $K$ e cada $\overline{K_{[0, r[}}$ são espaços de Koszmider, é suficiente provar que são fracamente Koszmider. Para isso, mostraremos que eles verificam as hipóteses do Teorema 2.18.

Como em (a)-(d) do Teorema 2.18, sejam $\left(f_{n}\right)_{n \in \mathbb{N}}$ uma sequência de funções contínuas de $K$ em $[0,1]$ com suportes contidos em abertos disjuntos, $\left(q_{l_{n}}\right)_{n \in \mathbb{N}}$ uma sequência relativamente discreta de pontos distintos de $Q=\left\{q_{n}: n \in \mathbb{N}\right\}$ tal que $f_{n}\left(q_{l_{m}}\right)=0$, para todo $m, n \in \mathbb{N}$, $\varepsilon>0$, e uma sequência limitada de medidas regulares $v_{n}$ em $K$ tais que $\left|\int f_{n} d v_{n}\right|>\varepsilon$. Vejamos que valem (e)-(g) do Teorema 2.18 .

Pelo Teorema de Tietze, existem extensões contínuas $\hat{f}_{n}:[0,1]^{2^{\omega}} \rightarrow[0,1]$ das funções $f_{n}$. Pelo Teorema de Mibu, veja Teorema 5.18, cada $\hat{f}_{n}$ depende apenas de um conjunto $A_{n} \subset 2^{\omega}$ enumerável de coordenadas, isto é, se $\left.y\right|_{A_{n}}=\left.z\right|_{A_{n}}$ então $\hat{f}_{n}(z)=\hat{f}_{n}(y)$. Como não existem conjuntos enumeráveis e cofinais em $2^{\omega}$, então existe $\gamma=\sup \cup A_{n}<2^{\omega}$. Assim, para todos $\alpha^{\prime}>\gamma$ e todo $n \in \mathbb{N}, \hat{f}_{n}$ permite definir funções contínuas $\hat{g}_{n}:[0,1]^{\alpha^{\prime}} \rightarrow[0,1]$ por $\hat{g}_{n}(x)=\hat{f}_{n}\left(\left.y\right|_{\alpha^{\prime}}\right)$.

Para todo $n \in \mathbb{N}$, sejam $\hat{v}_{n}$ as medidas em $[0,1]^{2^{\omega}}$ definidas por $\hat{v}_{n}[B]=v_{n}[B \cap K]$ para todo boreliano $B$ em $[0,1]^{\omega_{1}}$.

Pela enumeração feita no começo, existe $\alpha>\gamma$ tal que $f_{n}(\alpha)=\hat{f}_{n}, l_{n}(\alpha)=l_{n}, i(\alpha)=0$, $\mu_{n}(\alpha)=\left.\hat{v}_{n}\right|_{\mathcal{B}_{\alpha}}$. 
Como $\alpha>\gamma$, seja, para cada $n \in \mathbb{N}, \hat{g}_{n}:[0,1]^{\alpha} \rightarrow[0,1]$ definida a partir de $\hat{f}_{n}$, como antes, e defina $g_{n}:=\left.\hat{g}_{n}\right|_{K_{\alpha}}$. Temos $g_{n}\left(\left.q_{l_{m}}\right|_{\alpha}\right)=0$, para todo $m, n \in \mathbb{N}$, satisfazendo a condição E.3. Pela Proposição $1.3 \mathrm{e}$ - trocando $\gamma$, se necessário - o conjunto $\left\{\left.q_{l_{n}}\right|_{\alpha}: n \in \mathbb{N}\right\}$ é relativamente discreto em $K_{\alpha}$ para $\alpha>\gamma$, verificando a condição E.1. Pelo Lema 1.18 e a Proposição 1.19 temos

$$
\left|\int_{K_{\alpha}} g_{n} d \mu_{n}(\alpha)\right|=\left|\int_{K_{\alpha}} g_{n} d v_{n} \circ \pi_{\alpha}^{-1}\right|=\left|\int_{K} f_{n} d v_{n}\right|>\varepsilon,
$$

provando a condição E.4.

Pelo Lema 5.19, podemos assumir - trocando $\gamma$, se necessário - que $\left(g_{n}\right)_{n \in \mathbb{N}}$ são separadas por abertos disjuntos, satisfazendo a condição E.2. Concluímos que o passo $\alpha$ é não trivial.

Portanto, seguindo a construção, aplicamos o Lema 5.16 para $Q_{\alpha}=\left\{\left.q_{n}\right|_{\alpha}: n \in \mathbb{N}\right\}, \varepsilon(\alpha)$, $\left(\mu_{n}(\alpha)\right)_{n \in \mathbb{N}},\left(g_{n}\right)_{n \in \mathbb{N}},\left(l_{n}\right)_{n \in \mathbb{N}}$ e $\mathcal{P}_{\alpha}=\left\{\left(b_{\beta}^{i}, a_{\beta}^{i} \backslash b_{\beta}^{i}\right): i \in\{0,1\}, \beta<\alpha\right\}$. Consequentemente, existem $\delta>0, b \subset a \subset \mathbb{N}$ infinitos e funções $g_{n}^{\prime}: K_{\alpha} \rightarrow[0,1] \operatorname{com} \operatorname{supp}\left(g_{n}^{\prime}\right) \subset \operatorname{supp}\left(g_{n}\right)$ tais que

- $\left|\int g_{n}^{\prime} d \mu_{n}(\alpha)\right|>\delta$ e $\Sigma\left\{\int g_{m}^{\prime} d\left|\mu_{n}(\alpha)\right|: m \neq n, m \in a\right\}<\delta / 3$, para todo $n \in a$;

- $K_{\alpha}\left(\left(g_{n}^{\prime}\right)_{n \in b}\right)$ é uma extensão completa;

- $\overline{\left\{\left(\left.q_{l_{n}}\right|_{\alpha}\right)^{\prime}: n \in b\right\}} \cap \overline{\left\{\left(q_{l_{n}} \mid \alpha\right)^{\prime}: n \in a \backslash b\right\}} \neq \emptyset$, onde $q_{m}^{\prime}$ é definido como $\left(q_{m}, \Sigma_{n \in b} f_{n}^{\prime}\left(q_{m}\right)\right)$;

- $\overline{\left\{\left(\left.q_{n}\right|_{\alpha}\right)^{\prime}: n \in b_{\beta}^{i}\right\}} \cap \overline{\left\{\left(\left.q_{n}\right|_{\alpha}\right)^{\prime}: n \in a_{\beta}^{i} \backslash b_{\beta}^{i}\right\}} \neq \emptyset$, para todo $\beta<\alpha$ e $i \in\{0,1\}$.

Definimos, então, $K_{\alpha+1}:=K_{\alpha}\left(\left(g_{n}^{\prime}\right)_{n \in b}\right), a_{\alpha}^{i}:=a$ e $b_{\alpha}^{i}:=b$, para ambos $i \in\{0,1\}, Q_{\alpha+1}=$ $\left\{\left.q_{n}\right|_{\alpha} \frown t: n \in \mathbb{N}\right\}$, com $t=\sum_{n \in b} g_{n}^{\prime}\left(\left.q_{m}\right|_{\alpha}\right)$, e os $p_{n}^{\alpha+1}$ como uma extensão qualquer de $p_{n}^{\alpha}$. Note que, pela construção, $\left.q_{l_{n}}\right|_{\alpha^{\prime}}=q_{l_{n}}^{\alpha^{\prime}}$ e, para todo $\alpha^{\prime}>\alpha$, ocorre

$$
\overline{\left\{\left.q_{l_{n}}\right|_{\alpha^{\prime}}: n \in b_{\alpha}^{i}\right\}} \cap \overline{\left\{q_{l_{n}} \mid \alpha_{\alpha^{\prime}}: n \in a_{\alpha}^{i} \backslash b_{\alpha}^{i}\right\}} \neq \emptyset
$$

com $i \in\{0,1\}$ e o fecho tomado em $K_{\alpha^{\prime}}$. Isso implica, pelo Lema 5.17, que $\overline{\left\{q_{l_{n}}: n \in b_{\alpha}^{i}\right\}} \cap$ $\overline{\left\{q_{l_{n}}: n \in a_{\alpha}^{i} \backslash b_{\alpha}^{i}\right\}} \neq \emptyset$, com o fecho sendo tomado em $K$. Provamos, assim, a condição (g) do Teorema 2.18.

Vamos agora provar o item (e) de 2.18. Sejam $f_{n}^{\prime}: K \rightarrow[0,1]$, para $n \in \mathbb{N}$, as funções definidas por $g_{n}^{\prime} \circ \pi_{\alpha}$. Temos que cada $f_{n}^{\prime}$ é contínua e $\operatorname{supp}\left(f_{n}^{\prime}\right) \subset \operatorname{supp}\left(g_{n} \circ \pi_{\alpha}\right)=\operatorname{supp}\left(f_{n}\right)$. Pelo Lema 1.18 e a Proposição $1.19,\left|\int_{K} f_{n}^{\prime} d v_{n}\right|=\left|\int_{K_{\alpha}} g_{n}^{\prime} d \mu_{n}(\alpha)\right|>\delta$ e $\Sigma\left\{\int f_{m}^{\prime} d\left|v_{n}\right|: m \neq n, m \in a\right\}<\delta / 3$, para todo $n \in a_{\alpha}$.

Finalmente, pelo Lema 5.6, $\sup \left(g_{n}^{\prime}\right) \circ \pi_{\alpha}=\sup \left(f_{n}^{\prime}\right)$ em $C(K)$, provando (f) do Teorema 2.18. Adicionando a (e)-(g) o fato que para todo $0<r \leq 1, Q \cap \overline{K_{[0, r[}}$ é subespaço denso do fechado $\overline{K_{[0, r}[}$, podemos concluir do Teorema 2.18 que $K$ e $\overline{K_{[0, r}}$ para todo $\left.\left.r \in\right] 0,1\right]$ são fracamente Koszmider.

Por último, demonstramos que $K$ verifica a (e). Usamos o Lema 5.20. A sequência $\left\{p_{n} \in K\right.$ : $\left.\forall \alpha<\omega_{1}\left(\left.p_{n}\right|_{\alpha}=p_{n}^{\alpha}\right), n \in \mathbb{N}\right\}$ é relativamente discreta em $K$ pela construção. Seja $N \subset \mathbb{N}$ infinito, arbitrário, pela enumeração dada no começo existe $\alpha$ tal que $N(\alpha)=N$ e $i(\alpha)=2$. Pela construção,

$$
\overline{\left\{\left.p_{n}\right|_{\alpha+1}: n \in N(\alpha)\right\}} \cap \overline{\left\{\left.p_{n}\right|_{\alpha+1}: n \in \mathbb{N} \backslash N(\alpha)\right\}}=\emptyset
$$

em $K_{\alpha+1}$. Consequentemente, para todo $\alpha^{\prime}>\alpha$ temos $\overline{\left\{\left.p_{n}\right|_{\alpha^{\prime}}: n \in N(\alpha)\right\}} \cap \overline{\left\{\left.p_{n}\right|_{\alpha^{\prime}}: n \in \mathbb{N} \backslash N(\alpha)\right\}}=$ $\emptyset$, com o fecho tomado em $K_{\alpha^{\prime}}$, e, assim, $\overline{\left\{p_{n}: n \in N(\alpha)\right\}} \cap \overline{\left\{p_{n}: n \in \mathbb{N} \backslash N(\alpha)\right\}}=\emptyset$. Portanto, pelo 
Lema 5.20 o espaço $K$ contém um subespaço homeomorfo a $\beta \mathbb{N}$.

Para demostrar que quocientes do espaço construído no Teorema 6.1, que são da forma $C\left(\overline{K_{[0, r}}\right)$, não são isomorfos entre eles, usamos a seguinte propriedade dos espaços fracamente Koszmider:

Proposição 6.2. Se $K$ é um espaço fracamente Koszmider e $L$ é subespaço fechado próprio de $K$, então $C(K)$ não é isomorfo a $C(L)$.

Demonstração: Supondo que $L$ é subespaço fechado próprio de $K$, o operador $S: C(K) \rightarrow C(L)$ definido por $S(f)=\left.f\right|_{L}$ é sobrejetor e contínuo mas não injetor. Se $T: C(L) \rightarrow C(K)$ é um isomorfismo, então $T \circ S: C(K) \rightarrow C(K)$ é sobrejetor, porque $T$ e $S$ são sobrejetores, mas não um isomorfismo, porque $S$ não é injetor. Assim, pelo Teorema 2.3 de [Kos04], $K$ contém sequências convergentes ou $T \circ S$ não é multiplicador fraco, mas nos dois casos isso implica que $K$ não é fracamente Koszmider.

Demonstramos, agora, que o espaço do Teorema 6.1 tem pelo menos $2^{\omega}$ subespaços quocientes não isomorfos.

Teorema 6.3. Seja K como no Teorema 6.1. Então $C(K)$ tem $2^{\omega}$ quocientes indecomponíveis dois a dois não isomorfos.

Demonstração: Tome $K$ como no Teorema 6.1. Temos que cada $\overline{K_{[0, r}}$ é um espaço de Koszmider conexo e, em particular, $C\left(\overline{K_{[0, r}}\right)$ é indecomponível (ver Corolário 3.3). A aplicação $T: C(K) \rightarrow$ $C\left(\overline{K_{[0, r]}}\right)$ dada por $T(f)=\left.f\right|_{\overline{K_{[0, r}}}$ é uma transformação linear, contínua e sobrejetora, mostrando que $C\left(\overline{K_{[0, r]}}\right)$ é quociente de $C(K)$. Se $r<s, \overline{K_{[0, r]}} \subset \overline{K_{[0, s]}}$ e, portanto, pela Proposição 6.2, $C\left(\overline{K_{[0, r[}}\right)$ não é isomorfo a $C\left(\overline{K_{[0, s]}}\right)$.

Finalizamos este capítulo observando que para todo subconjunto aberto $V$ do espaço $K$ do Teorema 6.1 acontece que $\bar{V}$ é Koszmider.

Proposição 6.4. (ZFC+૪) Existe um espaço de Koszmider conexo K tal que, para todo conjunto aberto $V \subset K, \bar{V}$ é um espaço de Koszmider.

Demonstração: Sejam $K$ o compacto do Teorema 6.1 com $Q$ o subconjunto denso. Na prova de (d) do Teorema 6.1 se demonstrou que $K$ verifica as condições do Teorema 2.18. Como para todo $V \subset K$ aberto, $V \cap Q$ é denso em $\bar{V}$ então $\bar{V}$ é fracamente Koszmider. Note que por (b) do Teorema 6.1 o espaço $K$ não contém abertos borboleta então pelo Lema 2.10, $\bar{V}$ não contém abertos-borboleta. Logo, pelos Lemas 2.9 e 2.7, $\bar{V}$ é um espaço de Koszmider, para todo $V \subset K$ aberto.

Usando o Teorema 2.19, observamos que a construção do espaço conexo de Koszmider feita em [Kos04] também satisfaz a proposição 6.4, assumindo apenas a Hipótese do Contínuo, de modo que a Proposição acima também é verdadeira em $Z F C+C H$. Não sabemos se a construção de Plebanek, em [Ple04], também satisfaz essa proposição. Permanece em aberto se a Proposição 6.4 vale em ZFC. No entanto, usando a construção do espaço conexo fracamente Koszmider de [Kos04], feita em ZFC, obtemos a seguinte proposição. 
Proposição 6.5. Existe um espaço fracamente Koszmider conexo $K$ tal que, para todo conjunto aberto $V \subset K, \bar{V}$ é fracamente Koszmider.

Podemos conjeturar se o espaço fracamente Koszmider construído em [Kos04] - ou o espaço de Koszmider construído assumindo CH no mesmo artigo - também possui $2^{\omega}$ quocientes indecomponíveis não isomorfos. Todavia, a diferença fundamental entre as construções feitas em [Kos04] e a do Teorema 6.1 está na preservação da conexidade. Não sabemos se, nos espaços construídos em [Kos04], os subespaços $\overline{K_{[0, r[}}$ são conexos. O exemplo 4 sugere que possivelmente não. Dessa forma, permanece em aberto se o Teorema 6.1 vale em $\mathrm{ZFC}$ ou em $\mathrm{ZFC}+\mathrm{CH}$. 


\section{Capítulo 7}

\section{Um espaço não fracamente Koszmider e sem retrações não triviais}

Lembramos que uma retração em $K$ é uma função contínua $\varphi: K \rightarrow K$ tal que $\varphi(\varphi(x))=\varphi(x)$, para todo $x \in K$. A função identidade e as funções constantes são exemplos de retrações em $K \mathrm{e}$ são chamadas de retrações triviais.

As retrações em $K$ definem projeções em $C(K)$. De fato, se $\varphi: K \rightarrow K$ é uma retração, então $P: C(K) \rightarrow C(K)$ definida por $P(g)=g \circ \varphi$ é uma projeção. Em consequência as retrações em $K$ estão relacionadas com os subespaços complementados de $C(K)$. A compacidade de $K$ e a continuidade de $\varphi$ implicam que $\varphi(K)$ é fechado. Se $L=\varphi(K)$ então $P(C(K))$ é isometricamente isomorfo a $C(L)$ pela aplicação $\left.g \circ \varphi \mapsto g\right|_{L}$, portanto se $\varphi$ é uma retração não trivial em $K$ então $C(K)$ contém uma cópia complementada de $C(L)$ com $L=\varphi(K)$.

Neste capítulo construímos um exemplo de um espaço compacto separável $K$ satisfazendo as seguintes condições:

- não é fracamente Koszmider;

- é a união de dois espaços de Koszmider;

- não possui retrações não triviais.

Esse espaço é uma resposta parcial ao seguinte problema: existe um compacto $K$ não fracamente Koszmider tal que $C(K)$ é indecomponível? Não sabemos por enquanto se o espaço obtido neste capítulo responde afirmativamente a essa pergunta, visto que podemos ter projeções que não são dadas por retrações. Mas o espaço fornece um exemplo de $C(K)$ com uma "noção intermediária" de poucos operadores.

A construção do espaço é por indução transfinita, no passo sucessor o principal objetivo é fazer extensões completas que alternadamente evitam aparecer abertos-borboleta e operadores não multiplicadores fracos preservando um homeomorfismo entre os fechos de dois abertos disjuntos. Para isso, usaremos lemas demonstrados no Capítulo 5.

\subsection{Construção do espaço}

Algumas partes na construção e a prova dada aqui são exatamente como na construção no Teorema 6.1. Eventualmente iremos nos referir a essa prova para evitar repetições, mas mesmo 
assim iremos manter algumas por motivo de clareza.

Teorema 7.1. $(Z F C+\diamond)$ Existe um espaço compacto $K \subset[0,1]^{2^{\omega}}$ tal que:

(a) $K_{I}$ é infinito e conexo, para todo intervalo não degenerado $I \subset[0,1]$;

(b) existe $h: \overline{K_{\left[0, \frac{1}{3}[\right.}} \longrightarrow \overline{K_{\left.] \frac{2}{3}, 1\right]}}$ homeomorfismo tal que, para todo $x \in \overline{K_{\left[0, \frac{1}{3}[\right.}}$ e $\alpha<2^{\omega}, h(x)(\alpha)=$ $x(\alpha)$, se $\alpha>0$, e $h(x)(0)=1-x(0)$;

(c) $\overline{K_{[0, r[}}$ e $\overline{K_{11-r, 1]}}$ são espaços de Koszmider, para todo $\left.r \in\right] 0,2 / 3[$;

(d) se $M \subset[0,1]$ é raro, então $\pi^{-1}[M]$ é raro, em $K$;

(e) $K$ não contém abertos-borboleta, isto é, não existem abertos $V_{1}$ e $V_{2}$ disjuntos tais que $\bar{V}_{1} \cap \bar{V}_{2}$ é unitário;

(f) $K_{I} \backslash\{x\}$ é conexo, para todo intervalo aberto $I \subset[0,1]$ e todo $x$ tal que $x(0) \in I$;

Demonstração: Fixamos uma enumeração

$$
\left(\left(l_{n}(\alpha)_{n \in \mathbb{N}}\right),\left(f_{n}(\alpha)\right)_{n \in \mathbb{N}}, U(\alpha), V(\alpha), \varepsilon(\alpha),\left(\mu_{n}(\alpha)\right)_{n \in \mathbb{N}}, i(\alpha), j(\alpha), r(\alpha)\right)_{\alpha<\omega_{1}}
$$

com:

A.1. $\left(l_{n}(\alpha)\right)_{n \in \mathbb{N}}$ uma sequência crescente de inteiros positivos;

A.2. $f_{n}(\alpha):[0,1]^{\omega_{1}} \rightarrow[0,1]$ funções contínuas;

A.3. $U(\alpha)$ e $V(\alpha)$ uniões enumeráveis de abertos básicos em $[0,1]^{\omega_{1}}$;

A.4. $\varepsilon(\alpha)>0$;

A.5. $\left(\mu_{n}(\alpha)\right)_{n \in \mathbb{N}}$ uma sequência limitada de funções $\mu_{n}(\alpha): \mathcal{B}_{\alpha} \rightarrow \mathbb{R}$ que representam medidas de Radon em $[0,1]^{\alpha}$;

A.6. $r(\alpha) \in] \frac{1}{3}, \frac{2}{3}[$

A.7. $i(\alpha) \in 2$;

A.8. $j(\alpha) \in 2$;

tal que, dados $\beta<\omega_{1} \mathrm{e}$

B.1. $\left(l_{n}\right)_{n \in \mathbb{N}}$ uma sequência crescente de inteiros positivos;

B.2. $\left(f_{n}\right)_{n \in \mathbb{N}}$ uma sequência de funções contínuas de $[0,1]^{\omega_{1}}$ em $[0,1]$;

B.3. $U$ e $V$ uniões enumeráveis de abertos básicos em $[0,1]^{\omega_{1}}$;

B.4. Um número real $\varepsilon>0$;

B.5. $\left(\mu_{n}\right)_{n \in \mathbb{N}}$ uma sequência de funções de $\mathcal{B}_{\omega_{1}}$ em $\mathbb{R}$ que representam medidas de Radon;

B.6. $r \in] 1 / 3,2 / 3[$; 
B.7. $i \in 2$;

B.8. $j \in 2$;

existe $\alpha$ tal que $\beta<\alpha<\omega_{1}$ e

C.1. $l_{n}=l_{n}(\alpha)$, para todo $n \in \mathbb{N}$;

C.2. $f_{n}=f_{n}\left(\alpha_{0}\right)$, para todo $n \in \mathbb{N}$;

C.3. $U(\alpha)=U$ e $V(\alpha)=V$;

C.4. $\varepsilon(\alpha)=\varepsilon$;

C.5. $\mu_{n}(\alpha)=\left.\mu_{n}\right|_{\mathcal{B}_{\alpha}}$, para todo $n \in \mathbb{N}$, ou seja $\mu_{n} \circ \pi_{\alpha}^{-1}[B]=\mu_{n}(\alpha)[B]$ para todo $B \in \mathcal{B}_{\alpha}$ e todo $n \in \mathbb{N}$;

C.6. $r(\alpha)=r$;

C.7. $i(\alpha)=i$;

C.8. $j(\alpha)=j$.

Em um passo $\alpha<\omega_{1}$ supomos que temos construídos $K_{\alpha}, h_{\alpha},\left(a_{\beta}^{i}\right)_{\beta<\alpha, i \in 2},\left(b_{\beta}^{i}\right)_{\beta<\alpha, i \in 2}$ e $Q_{\alpha}=$ $\left\{q_{n}^{\alpha}: n \in \mathbb{N}\right\}$ tais que

D.1. $K_{\alpha} \subset[0,1]^{\alpha}$ é compacto e conexo;

D.2. $b_{\beta}^{i}$ e $a_{\beta}^{i} \backslash b_{\beta}^{i}$ são infinitos e tais que $b_{\beta}^{i} \subset a_{\beta}^{i} \subset \mathbb{N}$, para todos $i \in 2$ e $\beta<\alpha$;

D.3. $Q_{\alpha}$ é denso em $K_{\alpha}$ e $\left.q_{n}^{\alpha}\right|_{\beta}=q_{n}^{\beta}$ se $\beta<\alpha$;

D.4. $\overline{\left\{q_{n}^{\alpha}: n \in b_{\beta}^{i}\right\}} \cap \overline{\left\{q_{n}^{\alpha}: n \in a_{\beta}^{i} \backslash b_{\beta}^{i}\right\}} \neq \emptyset$, para todos $i \in 2$ e $\beta<\alpha$, onde os fechos estão sendo tomados em $K_{\alpha}$;

D.5. $h_{\alpha}:{\overline{\left(K_{\alpha}\right)}}_{\left[0, \frac{1}{3}[\right.} \longrightarrow{\overline{\left.\left(K_{\alpha}\right)_{]}, 0\right]}}_{3}$ é um homeomorfismo tal que $h_{\alpha}(x)(0)=1-x(0)$ e $h_{\alpha}(x)(\gamma)=$ $x(\gamma)$, para todos $x \in{\overline{\left(K_{\alpha}\right)}}_{\left[0, \frac{1}{3}[\right.}$ e $0<\gamma<\alpha$.

Para simplificar a notação, iremos denotar ${\overline{\left(K_{\alpha}\right)}}_{\left[0, \frac{1}{3}[\mathrm{e}\right.}{\overline{\left(K_{\alpha}\right)}}_{\left.\frac{2}{3}, 0\right]}$ por $K_{\alpha}^{1}$ e $K_{\alpha}^{3}$, respectivamente. Se $\alpha^{\prime}$ é ordinal limite, definimos $K_{\alpha^{\prime}}$ como o limite inverso de $K_{\alpha}$, para $\alpha<\alpha^{\prime}$. Os demais itens são definidos de maneira natural. Assim, por exemplo, $h_{\alpha^{\prime}}$ é definido por $h_{\alpha^{\prime}}(x)=y$ para $x \in K_{\alpha^{\prime}}^{1}$ se, e somente se, para todo $\alpha<\alpha^{\prime}, h_{\alpha}\left(\left.x\right|_{\alpha}\right)=\left.y\right|_{\alpha}$. Vejamos que vale D.5.

Para provarmos que $h_{\alpha^{\prime}}$ é injetora, sejam $x_{1} \neq x_{2}$ pontos em $K_{\alpha^{\prime}}^{1}$. Tome $\gamma<\alpha^{\prime}$ tal que $\pi_{\gamma}\left(x_{1}\right) \neq \pi_{\gamma}\left(x_{2}\right)$. Temos $h_{\gamma}\left(\pi_{\gamma}\left(x_{1}\right)\right) \neq h_{\gamma}\left(\pi_{\gamma}\left(x_{2}\right)\right)$, o que implica $h_{\alpha^{\prime}}\left(x_{1}\right) \neq h_{\alpha^{\prime}}\left(x_{2}\right)$. Para ver que $h_{\alpha^{\prime}}$ é sobrejetora, note que, como $\pi_{\alpha, \alpha^{\prime}}$ é contínua e $\left(K_{\alpha^{\prime}}\right)_{] 2 / 3,1]} \subset \pi_{\alpha, \alpha^{\prime}}^{-1}\left[K_{\alpha}^{3}\right]$, para todo $\alpha<\alpha^{\prime}$, então $K_{\alpha^{\prime}}^{3} \subset \pi_{\alpha, \alpha^{\prime}}^{-1}\left[K_{\alpha}^{3}\right]$, para todo $\alpha<\alpha^{\prime}$. Se $y \in K_{\alpha^{\prime}}^{3}$, temos $\left.y\right|_{\alpha} \in K_{\alpha}^{3}$, para todo $\alpha<\alpha^{\prime}$. Tomando $x$ o elemento em $K_{\alpha^{\prime}}^{1}$ tal que $\left.x\right|_{\alpha}=h_{\alpha}^{-1}\left(\left.y\right|_{\alpha}\right)$ temos $h_{\alpha^{\prime}}(x)=y$.

Para provar que $h_{\alpha^{\prime}}$ é contínua, sejam $x \in K_{\alpha^{\prime}}^{1}$ e $W$ um aberto básico que contém $h_{\alpha^{\prime}}(x)$, com $W=\prod_{\alpha<\alpha^{\prime}} V_{\alpha} \cap K_{\alpha^{\prime}}^{3}$ e $V_{\alpha} \neq[0,1]$ para apenas um número finito de $\alpha$ 's. Fixe $\gamma<\alpha^{\prime}$ tal que 
$\gamma>\max \left\{\alpha: V_{\alpha} \neq[0,1]\right\}$. Temos que, para toda $\alpha^{\prime}>\gamma$, a intersecção $\prod_{\alpha<\gamma} V_{\alpha} \cap K_{\gamma}^{3}$ é um aberto em $K_{\gamma}^{3}$ contendo $\pi_{\gamma, \alpha^{\prime}}\left(h_{\alpha^{\prime}}(x)\right)$.

Como $h_{\gamma}$ é homeomorfismo, $h_{\gamma}^{-1}\left[K_{\gamma}^{3} \cap \prod_{\alpha<\gamma} V_{\alpha}\right]$ é aberto em $K_{\gamma}^{1}$ contendo $\pi_{\gamma, \alpha^{\prime}}(x)$. Seja $O_{\gamma}$ aberto em $K_{\gamma}$ tal que $K_{\gamma}^{1} \cap O_{\gamma}=h_{\gamma}^{-1}\left[K_{\gamma}^{3} \cap \prod_{\alpha<\gamma} V_{\alpha}\right]$, então $W^{\prime}:=\pi_{\gamma, \alpha^{\prime}}^{-1}\left[O_{\gamma}\right] \cap K_{\alpha^{\prime}}^{1}$ é um aberto de $K_{\alpha^{\prime}}^{1}$ contendo $x$. Seja $u \in W^{\prime}$. Pela definição de $h_{\alpha^{\prime}}, \pi_{\gamma, \alpha^{\prime}}\left(h_{\alpha^{\prime}}(u)\right)=h_{\gamma}\left(\pi_{\gamma, \alpha^{\prime}}(u)\right) \in K_{\gamma}^{3} \cap \prod_{\alpha<\gamma} V_{\alpha}$. Logo pela forma como foi escolhido $\gamma, h_{\alpha^{\prime}}(u) \in K_{\alpha^{\prime}}^{3} \cap \prod_{\alpha<\alpha^{\prime}} V_{\alpha}$. Assim, $h_{\alpha^{\prime}}\left[W^{\prime}\right] \subset W$ e portanto $h_{\alpha^{\prime}}$ é contínua. A prova de que $h_{\alpha^{\prime}}^{-1}$ é contínua é análoga.

Observe que, pela definição de $h_{\alpha^{\prime}}$, se $x \in K_{\alpha^{\prime}}^{1}$, então $h_{\alpha^{\prime}}(x)(0)=\pi_{2, \alpha^{\prime}}\left(h_{\alpha^{\prime}}(x)\right)(0)=h_{2}\left(\pi_{2, \alpha^{\prime}}(x)\right)(0)=$ $1-\pi_{2, \alpha^{\prime}}(x)(0)$ e $h_{\alpha^{\prime}}(x)(\alpha)=h_{\alpha+1}(x)(\alpha)=\pi_{\alpha+1, \alpha^{\prime}}(x)(\alpha)$, para $\alpha^{\prime}>\alpha>0$.

Construído o compacto $K_{\alpha}$, diremos que $\alpha$ é um passo não trivial se:

$i(\alpha)=0$ e além de E.1 até E.4 como na construção do Teorema 6.1,

E.5. $g_{n}(x)=g_{n}\left(h_{\alpha}(x)\right)$, para todos $n \in \mathbb{N}$ e $x \in K_{\alpha}^{1}$;

E.6. $\mu_{n}(\alpha)(A)=0$, se $A \subset K_{\alpha} \backslash K_{\alpha}^{r}$, onde $K_{\alpha}^{r}=\overline{\left(K_{\alpha}\right)_{[0, r[}}$, se $j(\alpha)=0$, e $K_{\alpha}^{r}=\overline{\left(K_{\alpha}\right)_{] r, 1]}}$, se $j(\alpha)=1$.

ou $i(\alpha)=1$ e F.1 e F.2 como na prova do Teorema 6.1 .

Se $K_{\alpha}$ não verifica as condições acima dizemos que o passo é trivial e procedemos como na prova do Teorema 6.1. Neste caso $h_{\alpha+1}$ é definido por $h_{\alpha+1}(x)=\left(h_{\alpha}(x), 0\right)$.

No caso não trivial e $i(\alpha)=1$, aplicamos o Lema 5.12 para $K=K_{\alpha}, Q=Q_{\alpha}, h=h_{\alpha}$ homeomorfismo, $\mathcal{P}=\left\{\left(b_{\beta}^{i}, a_{\beta}^{i} \backslash b_{\beta}^{i}\right): \beta<\alpha, i \in 2\right\}$, e os abertos como sendo $\pi_{\alpha}[U(\alpha)] \cap K_{\alpha}$ e $\pi_{\alpha}[V(\alpha)] \cap K_{\alpha}$. Obtemos $b \subset \mathbb{N}$ infinito e coinfinito, sequências estritamente crescentes de naturais $\left(l_{n}^{i}\right)_{n \in \mathbb{N}}$ com $i \in\{0,1\}$, sequências de abertos $U_{n}$ e $V_{n}$ e uma sequência $\left(f_{n}\right)_{n \in \mathbb{N}}$ de funções duas a duas disjuntas que verificam $(\mathrm{d})$ até $(\mathrm{m})$ do Lema 5.12 .

Definimos $K_{\alpha+1}=K_{\alpha}\left(\left(f_{n}\right)_{n \in b}\right), Q_{\alpha+1}=\left\{q_{n}^{\alpha \frown t_{n}}: n \in \mathbb{N}\right\}$, onde $t_{n}=\sum_{m \in b} f_{m}\left(q_{n}^{\alpha}\right), a_{\alpha}^{1}=\left\{l_{n}^{i}:\right.$ $n \in b, i \in 2\}, b_{\alpha}^{1}:=\left\{l_{n}^{1}: n \in b\right\}, a_{\alpha}^{0}=\left\{l_{n}^{i}: n \in \mathbb{N} \backslash b, i \in 2\right\}, b_{\alpha}^{0}:=\left\{l_{n}^{1}: n \in \mathbb{N} \backslash b\right\}$, e $h_{\alpha+1}$ por $h_{\alpha+1}((x, t))=\left(h_{\alpha}(x), t\right)$ para $(x, t) \in K_{\alpha+1}^{1}$. Note que, em particular, por (k) do Lema 5.12, temos

$$
(x, i) \in \overline{\left\{q_{n}^{\alpha \frown} i: n \in b_{\alpha}^{i}\right\}} \cap \overline{\left\{q_{n}^{\alpha \frown} i: n \in a_{\alpha}^{i} \backslash \in b_{\alpha}^{i}\right\}},
$$

para $i \in\{0,1\}$ e com o fecho sendo tomado em $K_{\alpha+1}$. Assim,

$$
(x, 0),(x, 1) \in \overline{\pi_{\alpha, \alpha+1}^{-1}\left[\pi_{\alpha}[U(\alpha)] \cap K_{\alpha}\right]} \cap \overline{\pi_{\alpha, \alpha+1}^{-1}\left[\pi_{\alpha}[U(\alpha)] \cap K_{\alpha}\right]} .
$$

Note que $h_{\alpha+1}$ é bem definida: sejam $(x, t) \in K_{\alpha+1}^{1}$ e $W \times I$ um aberto contendo $\left(h_{\alpha}(x), t\right)$ onde $W$ é um aberto de $K_{\alpha}$ contendo $h_{\alpha}(x)$ e $I$ é um intervalo aberto de [0,1] contendo $t$. Como $h_{\alpha}$ é homeomorfismo, $h_{\alpha}^{-1}\left[W \cap K_{\alpha}^{3}\right]$ é aberto em $K_{\alpha}^{1}$. Seja $W^{\prime}$ um aberto de $K_{\alpha}$ contendo $x$ e tal que $W^{\prime} \cap K_{\alpha}^{1}=h_{\alpha}^{-1}\left[W \cap K_{\alpha}^{3}\right]$. Como $(x, t) \in W^{\prime} \times I$, temos $\left(W^{\prime} \times I\right) \cap\left(K_{\alpha+1}\right)_{[0,1 / 3[} \neq \emptyset$ e, portanto, existe $y \in W^{\prime} \cap D\left(\left(f_{n}\right)_{n \in b}\right)$ tal que $0 \leq y(0)<1 / 3$ e $\sum_{n \in b} f_{n}(y) \in I$. Como $y \in$ 
$\left(K_{\alpha}\right)_{[0,1 / 3[}$, existe um aberto $O_{y} \subset\left(K_{\alpha}\right)_{[0,1 / 3[}$ contendo $y$ que intersecta no máximo um número finito de suportes das $f_{n}$ 's. Logo, $h\left[O_{y}\right] \subset\left(K_{\alpha}\right)_{[2 / 3,1]}$ e, assim, $h(y) \in D\left(\left(f_{n}\right)_{n \in b}\right)$. Por (m) do Lema 5.12, $\sum_{n \in b} f_{n}(h(y))=\sum_{n \in b} f_{n}(y) \in I$ e, assim, $\left(h(y), \sum_{n \in b} f_{n}(h(y)) \in W \times I\right.$. Portanto $(h(x), t) \in K_{\alpha+1}^{3}$. A recíproca é análoga. Segue imediatamente da definição de $h_{\alpha+1}$ e do fato de que $h_{\alpha}: K_{\alpha}^{1} \rightarrow K_{\alpha}^{3}$ é homeomorfismo, que $h_{\alpha+1}$ é uma bijeção.

Para provarmos que $h_{\alpha+1}$ é contínua, sejam $(x, t) \in K_{\alpha+1}, W$ um aberto em $K_{\alpha}$ e $I$ um intervalo aberto em [0,1] tais que $\left(h_{\alpha}(x), t\right) \in(W \times I) \cap K_{\alpha+1}^{3}$. Como $h_{\alpha}$ é homemorfismo, $h_{\alpha}^{-1}\left[W \cap K_{\alpha}^{3}\right]$ é aberto em $K_{\alpha}^{1}$. Seja $W^{\prime}$ aberto em $K_{\alpha}$ tal que $W^{\prime} \cap K_{\alpha}^{1}=h_{\alpha}^{-1}\left[W \cap K_{\alpha}^{3}\right]$. Então $O_{x}:=\left(W^{\prime} \times I\right) \cap K_{\alpha+1}^{1}$ é aberto de $K_{\alpha+1}^{1}$ contendo $(x, t)$. Pela continuidade de $\pi_{\alpha, \alpha+1}, K_{\alpha+1}^{1} \subset \pi_{\alpha, \alpha+1}^{-1}\left[K_{\alpha}^{1}\right]$. Logo, se $(z, s) \in O_{x}$, então $z \in h_{\alpha}^{-1}\left[W \cap K_{\alpha}^{3}\right]$. Assim $\left(h_{\alpha}(z), s\right) \in K_{\alpha+1}^{3}$ e $h_{\alpha}(z) \in W \cap K_{\alpha+1}^{3}$, provando que $h_{\alpha}\left[O_{x}\right] \subset(W \times I) \cap K_{\alpha+1}$ e, portanto, que $h_{\alpha+1}$ é contínua.

Faremos agora a construção no caso não trivial e $i(\alpha)=0$. Por $C H$ (que segue de $\diamond$ ), temos $|\alpha| \leq$ $\omega$ e, portanto, $K_{\alpha}$ é metrizável. Aplicando o Lema 5.15 para $K_{\alpha}, Q_{\alpha}, r(\alpha), h_{\alpha}, \varepsilon(\alpha),\left(\mu_{n}(\alpha)\right)_{n \in \mathbb{N}}$, $\left(g_{n}\right)_{n \in \mathbb{N}},\left(l_{n}\right)_{n \in \mathbb{N}}$ e $\mathcal{P}_{\alpha}=\left\{\left(b_{\beta}^{i}, a_{\beta}^{i} \backslash b_{\beta}^{i}\right): i \in\{0,1\}, \beta<\alpha\right\}$, existem $\delta>0$, funções contínuas $g_{n}^{\prime}: K_{\alpha} \rightarrow[0,1] \operatorname{com} \operatorname{supp}\left(f_{n}^{\prime}\right) \subset\left(f_{n}\right)$, e subconjuntos infinitos $a, b^{\prime}$ de $\mathbb{N}$ tais que $b^{\prime} \subset a$ e, para todo $b \subset b^{\prime}$, acontecem (f) até (j) do Lema 5.15.

Fixamos $b \subset b^{\prime}$ tal que $a \backslash b$ é infinito e definimos $K_{\alpha+1}:=K_{\alpha}\left(\left(g_{n}^{\prime}\right)_{n \in b}\right), a_{\alpha}^{i}:=a$ e $b_{\alpha}^{i}:=b$, para $i \in\{0,1\}, Q_{\alpha+1}=\left\{q_{n}^{\alpha \frown t}: n \in \mathbb{N}\right\}$, com $t=\sum_{m \in b} g_{m}^{\prime}\left(q_{n}^{\alpha}\right)$, e $h_{\alpha+1}\left(x^{\frown} t\right)=\left(h_{\alpha}(x)^{\frown} t\right)$, para todo $x^{\frown} t \in K_{\alpha+1}^{1}$. A prova de que $h_{\alpha+1}: K_{\alpha+1}^{1} \rightarrow K_{\alpha+1}^{3}$ é homeomorfismo é como para o caso $i(\alpha)=1$ mas usando E.5. no lugar de (m) do Lema 5.12.

Isso completa a construção no caso ordinal sucessor.

Agora definimos $K=K_{\omega_{1}}, Q=Q_{\omega_{1}}:=\left\{q_{n}: n \in \mathbb{N}\right\}, h=h_{\omega_{1}}$ e demonstramos que verificam o teorema. Denotaremos por $K_{1}$ e $K_{3}$ os conjuntos $\overline{K_{\left[0, \frac{1}{3}[\right.}}$ e $\overline{K_{\left.] \frac{2}{3}, 1\right]}}$, respectivamente.

O item (b) segue imediatamente da construção. A prova dos itens (a), (d) e (e) são a mesma que foi dada na prova do Teorema 6.1. A prova de (f) é consequência imediata do Lema 2.11 e a parte (e). Falta provar o item (c), que faremos através do Teorema 2.18. A prova de que $\overline{K_{[0, r[}}$ e $\overline{K_{11-r, 1]}}$ são fracamente Koszmider, para $r \in[0,2 / 3$ [, é análoga pelo que apresentaremos apenas para $\overline{K_{[0, r[}}$. Note que $Q \cap \overline{K_{[0, r[}}$ é denso em $\overline{K_{[0, r}}$, para todo $\left.r \in\right] 0,2 / 3[$, porque a interseção de qualquer aberto de $K \operatorname{com} K_{[0, r[}$ é aberto em $K$. Assim é suficiente provar $\overline{K_{[0, r[}}$ e $Q_{r}:=Q \cap \overline{K_{[0, r[}}$ verificam as hipóteses desse teorema para $r \in] 1 / 3,2 / 3[$.

Fixemos $r \in] 1 / 3,2 / 3\left[\right.$ arbitrário. Como em (a)-(d) do Teorema 2.18, sejam $\left(f_{n}\right)_{n \in \mathbb{N}}$ uma sequência de funções contínuas de $\overline{K_{[0, r[}}$ em [0,1] com suportes contidos em abertos disjuntos, $\left(q_{l_{k}}\right)_{k \in \mathbb{N}}$ uma sequência de pontos em $Q_{r}$ relativamente discreta tal que $f_{n}\left(q_{l_{m}}\right)=0$ para todo $m, n \in \mathbb{N}, \varepsilon>0$, e $\left(\nu_{k}\right)_{k \in \mathbb{N}}$ uma sequência de medidas regulares sobre $\overline{K_{[0, r[}}$ tais que $\left|\int_{\overline{K_{[0, r[}}} f_{n} d \nu_{n}\right|>\varepsilon$.

Vejamos que acontecem (e)-(g) do Teorema 2.18. Seja $\check{f}_{n}: \overline{K_{[0, r[}} \cup K_{3} \rightarrow[0,1]$ a função definida por

$$
\check{f}_{n}=\left\{\begin{array}{lll}
f_{n} & \text { se } & x \in \overline{K_{[0, r[}} \\
f_{n} \circ h^{-1} & \text { se } & x \in K_{3}
\end{array}\right.
$$

Notemos que $\check{f}_{n}$ é uma extensão contínua de $f_{n}$ ao fechado $\overline{K_{[0, r}} \cup K_{3}$. Pela normalidade de $K$ e o Lema 2.17, existem extensões contínuas $\tilde{f}_{n}: K \rightarrow[0,1]$ das $\check{f}_{n}$ 's com suportes contidos em abertos disjuntos e $\left.\left(\tilde{f} \circ h^{-1}\right)\right|_{K_{3}}=\left.\tilde{f}_{n}\right|_{K_{1}}$. 
Pelo Teorema de Tietze, existem extensões contínuas $\hat{f}_{n}:[0,1]^{2^{\omega}} \rightarrow[0,1]$ das funções $\tilde{f}_{n}$. Pelo Teorema de Mibu, veja Teorema 5.18, cada $\hat{f}_{n}$ depende apenas de um conjunto $A_{n} \subset 2^{\omega}$ enumerável de coordenadas, isto é, se $\left.y\right|_{A_{n}}=\left.z\right|_{A_{n}}$ então $\hat{f}_{n}(z)=\hat{f}_{n}(y)$. Como não existem conjuntos enumeráveis e cofinais em $2^{\omega}$, então existe $\gamma=\sup \cup A_{n}<2^{\omega}$. Assim, para todos $\alpha^{\prime}>\gamma$ e todo $n \in \mathbb{N}, \hat{f}_{n}$ permite definir funções contínuas $\hat{g}_{n}:[0,1]^{\alpha^{\prime}} \rightarrow[0,1]$ por $\hat{g}_{n}(x)=\hat{f}_{n}\left(\left.y\right|_{\alpha^{\prime}}\right)$.

Para todo $n \in \mathbb{N}$, sejam $\tilde{\nu}_{n}$ a medida em $K$ definida por $\tilde{\nu}_{n}[A]=\nu_{n}\left[A \cap \overline{K_{[0, r}}\right]$, para todo $A$ boreliano em $K$, e $\hat{\nu}_{n}$ as medidas em $[0,1]^{\omega_{1}}$ definidas por $\hat{\nu}_{n}[B]=\tilde{\nu}_{n}[B \cap K]$ para todo $B$ boreliano de $[0,1]^{\omega_{1}}$.

Pela enumeração dada no começo, existe $\alpha>\gamma$ tal que $f_{n}(\alpha)=\hat{f}_{n}, l_{n}(\alpha)=l_{n}, i(\alpha)=0$, $j(\alpha)=0, r(\alpha)=r$ e $\mu_{n}(\alpha)=\left.\hat{\nu}_{n}\right|_{\mathcal{B}_{\alpha}}$.

Pela Proposição 1.3 e - trocando $\gamma$, se necessário - o conjunto $\left\{\left.q_{l_{n}}\right|_{\alpha}: n \in \mathbb{N}\right\}$ é relativamente discreto em $K_{\alpha}$ para $\alpha>\gamma$, verificando a condição E.1. Para cada $n \in \mathbb{N}$, seja $g_{n}:=\left.\hat{g}_{n}\right|_{K_{\alpha}}$ onde $\hat{g}_{n}$ é definida como antes. Pelo Lema 5.19, podemos assumir - trocando $\gamma$, se necessário - que $\left(g_{n}\right)_{n \in \mathbb{N}}$ são separadas por abertos disjuntos, satisfazendo a condição E.2. Adicionalmente, $g_{n}\left(\left.q_{l_{m}}\right|_{\alpha}\right)=0$, para todo $m, n \in \mathbb{N}$, satisfazendo a condição E.4.

Pelo Lema 1.18 e a Proposição 1.19 temos

$$
\left|\int_{K_{\alpha}} g_{n} d \mu_{n}(\alpha)\right|=\left|\int_{K_{\alpha}} g_{n} d \tilde{\nu}_{n} \circ \pi_{\alpha}^{-1}\right|=\left|\int_{\overline{K_{[0, r]}}} f_{n} d \nu_{n}\right|>\varepsilon,
$$

provando a condição E.5.

A condição E.3 segue da definição das $g_{n}$ 's e a condição E.6 segue da definição de $\hat{\nu}_{n}$ acima. Portanto, concluímos que o passo $\alpha$ é não trivial.

Seguindo a construção, aplicamos o Lema 5.15 para $Q_{\alpha}=\left\{\left.q_{n}\right|_{\alpha}: n \in \mathbb{N}\right\}, \varepsilon(\alpha),\left(\mu_{n}(\alpha)\right)_{n \in \mathbb{N}}$, $\left(g_{n}\right)_{n \in \mathbb{N}},\left(l_{n}\right)_{n \in \mathbb{N}}, h(\alpha)$ e $\mathcal{P}_{\alpha}=\left\{\left(b_{\beta}^{i}, a_{\beta}^{i} \backslash b_{\beta}^{i}\right): i \in\{0,1\}, \beta<\alpha\right\}$. Consequentemente, existem $\delta>0$, $b \subset a \subset \mathbb{N}$ infinitos e funções $g_{n}^{\prime}: K_{\alpha} \rightarrow[0,1] \operatorname{com} \operatorname{supp}\left(g_{n}^{\prime}\right) \subset \operatorname{supp}\left(g_{n}\right)$ que verificam (f) até (j) do Lema 5.15 e $\left.q_{l_{n}}\right|_{\alpha}=q_{l_{n}}^{\alpha}$.

Portanto, $K_{\alpha+1}:=K_{\alpha}\left(\left(g_{n}^{\prime}\right)_{n \in b}\right)$ é uma extensão completa, $a_{\alpha}^{i}:=a$ e $b_{\alpha}^{i}:=b$, para ambos $i \in\{0,1\}, Q_{\alpha+1}=\left\{\left.q_{n}\right|_{\alpha} \frown t: n \in \mathbb{N}\right\}$, com $t=\sum_{n \in b} g_{n}^{\prime}\left(\left.q_{m}\right|_{\alpha}\right)$.

Note que, pela construção, para todo $\alpha^{\prime}>\alpha,\left.q_{l_{n}}\right|_{\alpha^{\prime}}=q_{l_{n}}^{\alpha^{\prime}}$ e

$$
\overline{\left\{\left.q_{l_{n}}\right|_{\alpha^{\prime}}: n \in b_{\alpha}^{i}\right\}} \cap \overline{\left\{\left.q_{l_{n}}\right|_{\alpha^{\prime}}: n \in a_{\alpha}^{i} \backslash b_{\alpha}^{i}\right\}} \neq \emptyset
$$

com $i \in\{0,1\}$ e o fecho tomado em $K_{\alpha^{\prime}}$. Isso implica, pelo Lema 5.17, que $\overline{\left\{q_{l_{n}}: n \in b_{\alpha}^{i}\right\}} \cap$ $\overline{\left\{q_{l_{n}}: n \in a_{\alpha}^{i} \backslash b_{\alpha}^{i}\right\}} \neq \emptyset$, com o fecho sendo tomado em $K$. Como $q_{l_{n}} \in Q_{r}$ para todo $n \in \mathbb{N}$ e $\overline{K_{[0, r[}}$ é fechado (contendo $Q_{r}$ ) então a intersecção anterior está contida em $\overline{K_{[0, r}}$. Assim temos provada a condição $(\mathrm{g})$ do Teorema 2.18 para $b$ e $a$ obtidos no passo $\alpha$.

Agora, sejam $f_{n}^{\prime}: K \rightarrow[0,1]$, para $n \in \mathbb{N}$, as funções definidas por $g_{n}^{\prime} \circ \pi_{\alpha}$. Temos que cada $f_{n}^{\prime}$ é contínua e $\operatorname{supp}\left(f_{n}^{\prime}\right) \subset \operatorname{supp}\left(g_{n} \circ \pi_{\alpha}\right)=\operatorname{supp}\left(f_{n}\right)$. Vejamos que $\delta, a, b$ e $\left.f_{n}^{\prime}\right|_{K_{[0, r[}}$ verificam (e) e (f) do Teorema 2.18. Como para cada $n \in a_{\alpha}^{i}$, a função $g_{n}^{\prime}$ verifica (f) do Lema 5.15, pelo Lema 1.18 e a Proposição 1.19 ,

$$
\left|\int_{K_{[0, r]}} f_{n}^{\prime}\right|_{\bar{K}_{[0, r[}} d \nu_{n}|=| \int_{K} f_{n}^{\prime} d \tilde{\nu}_{n}|=| \int_{K_{\alpha}} g_{n}^{\prime} d \mu_{n}(\alpha) \mid>\delta
$$


e $\sum\left\{\left.\int_{\overline{K_{[0, r[}}} f_{m}^{\prime}\right|_{\overline{K_{[0, r[}}} d\left|\nu_{n}\right|: m \neq n, m \in a\right\}<\delta / 3$, para todo $n \in a$, garantindo (e) do Teorema 2.18.

Finalmente, pelo Lema 5.6, $\sup \left(g_{n}^{\prime}\right) \circ \pi_{\alpha}=\sup \left(f_{n}^{\prime}\right)$ em $C(K)$ e, $\left.\operatorname{comosup}\left(f_{n}^{\prime}\right)\right|_{\overline{K_{[0, r \mid}}}=\sup \left(\left.f_{n}^{\prime}\right|_{\overline{K_{[0, r]}}}\right)$ em $C\left(\overline{K_{[0, r}}\right)$, temos satisfeita a condição (f) do Teorema 2.18. Adicionando a (e)-(g) o fato que para todo $0<r<s \leq 1, Q_{s} \cap \overline{K_{[0, r}}$ é subespaço denso do fechado $\overline{K_{[0, r}}$, podemos concluir do Teorema 2.18 que $\overline{K_{[0, r}[}$ para todo $\left.r \in\right] 0, \frac{2}{3}$ [ são fracamente Koszmider e, portanto - pelo item (e) e os Lemas 2.9, 2.7 e 2.10 - são espaços de Koszmider, completando a prova de (c).

Teorema 7.2. O espaço $K$ satisfazendo as condições do Teorema 7.1 não contém retrações não triviais.

Demonstração: Sendo $K$ como no enunciado do Teorema 7.1, introduzimos as seguintes notações:

$$
\begin{aligned}
& V_{1}:=K_{\left[0, \frac{1}{3}[\right.} ; \\
& V_{2}:=K_{] \frac{1}{3}, \frac{2}{3}} ; \\
& V_{3}:=K_{] \frac{2}{3}, 1\right]} ; \\
& K_{i}:=\overline{V_{i}} ; \\
& V_{12}=K_{\left[0, \frac{2}{3}[\right.} ; \\
& V_{23}=K_{] \frac{1}{3}, 1\right]} ; \\
& K_{i j}=K_{i} \cup K_{j} ; \\
& \tilde{K}_{1}=K_{1} \cup K_{\left\{\frac{1}{3}\right\}} ; \\
& \tilde{K}_{3}=K_{3} \cup K_{\left\{\frac{2}{3}\right\}} ;
\end{aligned}
$$

Afirmação 7.1. $K=K_{1} \cup K_{2} \cup K_{3}$.

A afirmação segue do fato de que $K_{\left\{\frac{1}{3}, \frac{2}{3}\right\}}$ é raro e, portanto, $K_{\left\{\frac{1}{3}, \frac{2}{3}\right\}} \backslash K_{1} \cup K_{2} \cup K_{3}=\emptyset$.

Afirmação 7.2. $K_{12}=\bar{V}_{12}$ e $K_{23}=\bar{V}_{23}$.

A demonstração é análoga à da afirmação 7.1

Afirmação 7.3. $\left|\overline{V_{12}} \cap K_{3}\right| \geq 2$.

Primeiro tomemos $\overline{V_{12}} \cup K_{3}=K$. De fato, essa união é fechada e, portanto, o complemento é aberto. Como $K \backslash \overline{V_{12}} \cup K_{3} \subset K_{\left\{\frac{2}{3}\right\}}$, isso iria contradizer com o fato desse último conjunto ser raro. Em particular, $K_{1} \cup K_{2} \cup K_{3}=K$.

Se tivéssemos $\overline{V_{12}} \cap K_{3}=\emptyset$, teríamos $K$ desconexo. Se essa intersecção fosse unitária, isso iria contradizer a condição $(f)$, sobre não existência de abertos-borboleta.

Afirmação 7.4. $\left|\overline{V_{23}} \cap K_{1}\right| \geq 2$.

Essa afirmação é análoga à 7.3. 
Afirmação 7.5. Para $i \in\{1,2\},\left|K_{i} \cap K_{i+1}\right| \geq 2$.

Análoga à afirmação 7.3 .

Note que, se $x \in K_{1} \backslash V_{1}$, então $x(0)=\frac{1}{3}$. Além disso, para cada $x \in K_{\left\{\frac{1}{3}\right\}}$, temos que $x \in K_{1}$ se, e somente se, $x^{\prime} \in K_{3}$, onde $x^{\prime}(0)=\frac{2}{3}$ e $x^{\prime}(\alpha)=x(\alpha)$, para $\alpha>0$. Dessa forma, podemos estender $h$ para um homeomorfismo entre $K_{1}$ e $K_{3}$ que associa $x$ a $x^{\prime}$. Por simplicidade, usaremos a mesma notação $h$ para representar a extensão. Note ainda que a extensão satisfaz a mesma propriedade de que $h(x)(0)=1-x(0)$ e $h(x)(\alpha)=x(\alpha)$ para $\alpha>0$.

Seja $f: K \longrightarrow K$ uma retração. Mostraremos que $f$ é constante ou a identidade.

Afirmação 7.6. Se existe $x_{0}$ tal que $x_{0} \neq f\left(x_{0}\right)$ e ambos pertencem a $V_{12}$, então $\left.f\right|_{V_{12}}$ é constante.

Para provarmos a afirmação, chamaremos $f\left(x_{0}\right)$ de $y_{0}$, fixaremos $z \in V_{12}$ e provaremos que $f(z)=y_{0}$. Tome $r<s<\frac{2}{3}$ tal que $r>x_{0}(0), y_{0}(0), z(0)$, e defina $L=\overline{K_{[0, s}}$, que, pela construção, é fracamente Koszmider. Pela Proposição 2.14 o conjunto $f^{-1}\left[\left\{y_{0}\right\}\right] \cap\left(L \backslash\left\{y_{0}\right\}\right)$ é aberto (e obviamente fechado) em $L \backslash\left\{y_{0}\right\}$. Pela conexidade de $L \backslash\left\{y_{0}\right\}$ concluímos que $f^{-1}\left[\left\{y_{0}\right\}\right] \cap L$ é vazio ou todo $L$. Como $x_{0}$ pertence ao conjunto, não pode ser vazio. Assim, concluímos, em particular, que $f(z)=y_{0}$, visto que $z \in L$.

Afirmação 7.7. Se existe $x_{0}$ tal que $x_{0} \neq f\left(x_{0}\right)$ e ambos pertencem a $V_{23}$, então $\left.f\right|_{V_{23}}$ é constante.

Demonstração análoga à da afirmação 7.6.

Afirmação 7.8. $\left.f\right|_{K_{2}}$ é constante ou a identidade.

Da afirmação 7.6 segue que $\left.f\right|_{V_{2}}$ é constante ou a identidade. Como $V_{2}$ é denso em $K_{2}$, temos que $\left.f\right|_{K_{2}}$ é constante ou a identidade.

Afirmação 7.9. Se $f$ não é constante, para cada $i \in\{1,3\}$ existe $j \in\{1,3\}$ tal que $f\left[K_{i}\right] \subset \tilde{K}_{j}$.

Provaremos a afirmação para $i=1$, sendo que para $i=3$ é análogo. Se $f\left[K_{1}\right] \cap V_{2} \neq \emptyset$ temos, pela afirmação 7.6, que $\left.f\right|_{V_{12}}$ é constante. Em particular, $\left.f\right|_{K_{12}}$ é constante. Caso contrário, temos $f\left[K_{1}\right] \subset \tilde{K}_{1} \cup \tilde{K}_{3}$. Pela conexidade de $K_{1}$ temos $f\left[K_{1}\right] \subset \tilde{K}_{j}$, para algum $j \in\{1,3\}$. Logo, se $f\left[K_{i}\right] \cap V_{2} \neq \emptyset$ para ambos $i \in\{1,3\}$, temos que $f$ é constante. Se tivéssemos essa condição verdadeira para apenas um $i \in\{1,3\}$, teríamos que $\operatorname{Im}(f)=\tilde{K}_{j} \cup\{y\}$, para algum $j \in\{1,3\}$ e algum $y \in V_{2}$, contradizendo a conexidade de $K$ e continuidade de $f$. Logo, vale $f\left[K_{i}\right] \cap V_{2}=\emptyset$ para ambos $i \in\{1,3\}$, concluindo a afirmação.

Afirmação 7.10. Se $\left.f\right|_{K_{2}}$ é a identidade, então $f$ é a identidade.

Como $K_{2}$ não é unitário, temos que $f$ não é constante e, pela afirmação 7.9 , temos $f\left[K_{1}\right] \subset \tilde{K}_{1}$ ou $f\left[K_{1}\right] \subset \tilde{K}_{3}$. Pela afirmação 7.5, temos que $K_{1} \cap K_{2} \neq \emptyset$. Tomando $x \in K_{1} \cap K_{2}$, temos $f(x)=x \in K_{1} \subset \tilde{K}_{1}$. Portanto, $f\left[K_{1}\right] \subset \tilde{K}_{1} \subset V_{12}$ e, pela afirmação $7.6,\left.f\right|_{K_{1}}$ é constante ou a identidade. Mas, pela afirmação 7.5, não pode ocorrer o primeiro caso, visto que $K_{1} \cap K_{2}$ tem pelo menos dois elementos e a restrição de $f$ nesse conjunto é a identidade, que é injetora. Logo, $\left.f\right|_{K_{1}}$ é a identidade e, analogamente, $\left.f\right|_{K_{3}}$ também é a identidade. Assim, da afirmação 7.1 concluímos que $f$ é a identidade.

Afirmação 7.11. Se $\left.f\right|_{K_{2}}$ é constante, então $f$ é constante. 
Assumiremos que $f$ não é constante. Como fizemos na afirmação anterior, iremos provar que $\left.f\right|_{K_{1}}$ é constante, sendo a prova de que $\left.f\right|_{K_{3}}$ é constante análoga e suficiente para chegarmos a um absurdo.

Seja $\{y\}=f\left[K_{2}\right]$. Pela afirmação $7.9, f[K] \subset \tilde{K}_{1} \cup \tilde{K}_{3} \cup\{y\}$. Logo, pela conexidade de $f[K]$, não podemos ter $y \in V_{2}$. Logo, $y \in \tilde{K}_{j}$, para algum $j \in\{1,3\}$.

Suponha $y \in \tilde{K}_{1}$. Pela afirmação 7.5 temos que $K_{1} \cap K_{2} \backslash\{y\} \neq \emptyset$. Tomando $x$ nessa intersecção, temos $f(x)=y$, ambos são distintos e pertencentes a $\tilde{K}_{1} \subset V_{12}$, concluindo, pela afirmação 7.6, que $\left.f\right|_{K_{1}}$ é constante.

Suponha $y \in \tilde{K}_{3}$. Pela afirmações 7.9 e 7.5 temos que $f\left[K_{1}\right] \subset \tilde{K}_{3}$. Pela afirmação 7.7 temos que $\left.f\right|_{V_{23}}$ é constante igual a $y$. Logo, se $x \in K_{1}$, temos $f(x) \in \tilde{K}_{3} \subset V_{23}$ e, portanto, $y=f(f(x))=$ $f(x)$, provando que $\left.f\right|_{K_{1}}$ é constante.

Corolário 7.3. Existe um compacto $K$ não fracamente Koszmider que é união finita de espaços de Koszmider e não possui retração não trivial. 


\section{Capítulo 8}

\section{Conclusões}

Desde que Koszmider em 2004, em [Kos04], apresentou os primeiros exemplos de um espaço compacto $K$ tal que $C(K)$ tem poucos operadores, tanto no sentido de que todos os operadores em $C(K)$ são multiplicações fracas quanto no que todos são multiplicadores fraco - espaços esses que posteriormente foram chamados de espaços Koszmider e fracamente Koszmider, respectivamente -, esses se tornaram um objeto de pesquisa bastante promissor. A presente tese contém alguns avanços no estudo desses espaços e apresenta um resumo do que foi mostrado por outros autores. No entanto, muito continua sendo desconhecido sobre esse tema. Contemplamos, neste capítulo, algumas investigações futuras sobre esses espaços.

No Capítulo 2, a caraterização dos operadores multiplicadores fracos usando sequências de funções contínuas com suportes contidos em abertos disjuntos, no lugar de sequências de funções duas a duas disjuntas, permite estender as condições suficientes para um espaço $K$ ser fracamente Koszmider a alguns subespaços. Isso se deve em parte ao fato de que conjuntos enumeráveis de funções com suporte contido em abertos disjuntos contidos num subespaço fechado de $K$ podem ser estendidas a $K$ preservando essa caraterística, como se detalhou nesse mesmo capítulo. Na literatura encontra-se uma prova geral de que as funções pertencentes a uma família enumerável de as funções contínuas definidas num subespaço fechado de $K$, e duas a duas disjuntas, podem ser estendidas continuamente a $K$ preservando a propriedade de serem duas a duas disjuntas (veja Corolário 11 em [BD02]). Mas, com a caracterização que provamos, oferecemos uma demonstração direta e mais simples do que utilizando aquele resultado.

Koszmider, em [Kos04], demonstrou que os espaços fracamente Koszmider que não contêm abertos-borboleta são Koszmider. No Capítulo 2 provamos que os fechos de abertos herdam a propriedade de não possuir abertos-borboleta. Assim, no caso em que um espaço Koszmider $K$ verifique as condições do Teorema 2.18, é possível concluir que todo fecho de um subconjunto aberto de $K$ é um espaço de Koszmider.

Uma consequência imediata dos trabalhos de Koszmider em [Kos04] e Schlackow em [Sch08a] é que, se um espaço compacto conexo é Koszmider, então $C(K)$ é indecomponível. No Capítulo 3 se observou que esses trabalhos também permitem deduzir que a recíproca da afirmação anterior não acontece em geral. Ou seja, existe um espaço $C(K)$ indecomponível tal que $K$ não é Koszmider. No Capítulo 7, usando o axioma $\diamond$, foi construído um exemplo de um espaço compacto e não fracamente Koszmider $K$ tal que $C(K)$ não tem projeções geradas por retrações não triviais em $K$. Este resultado é uma resposta parcial a questão sobre $C(K)$ indecomponível implicar $K$ fracamente Koszmider. 
Baseados nos trabalhos de Fajardo em [Faj10] e Schlackow em [Sch08a], no Capítulo 3 apresentamos um exemplo de um espaço fracamente Koszmider que é decomponível. Pelo Corolário 1.10, devido a Pełczyński, os subespaços complementados de $C(K)$ de dimensão infinita contêm um subespaço isomorfo a $c_{0}$. Portanto, pelo Teorema de Sobczyk (ver Teorema 1.5), o Teorema 1.6, e a Proposição 2.16 se $K$ é fracamente Koszmider nenhum subespaço complementado de $C(K)$ é separável.

Os resultados do Capítulo 3 ainda permitem estabelecer que, se $K$ é fracamente Koszmider, os subespaços complementados de $C(K)$ que são da forma $C(L)$ são fracamente Koszmider. Neste caso não é assumida nenhuma relação entre $L$ e $K$. Além disso, é possível afirmar que nenhum subespaço complementado é isomorfo ao seu complementar. Assim, os resultados do Capítulo 3 poderiam ser uma ferramenta para obter novos espaços fracamente Koszmider a partir de outros.

Desde o surgimento dos conceitos de lineabilidade e espaçabilidade, no começo da década passada, uma extensa quantidade de trabalhos tem usado essa terminologia para descrever o "tamanho linear" de um subconjunto de um espaço vetorial ou espaço vetorial topológico. O conjunto $\operatorname{NWM}(C(K))$ dos operadores não multiplicadores fracos sobre $C(K)$ foi estudado com este enfoque por Fajardo et al., em 2013, em [FKP13]. Nesse artigo foi provado que, se $K$ não é fracamente Koszmider, existe um conjunto enumerável de operadores não multiplicadores fracos em $\mathcal{L}(C(K))$, linearmente independentes e tais que o fecho do espaço gerado por eles está contido em $\operatorname{NWM}(C(K)) \cup\{0\}$, concluindo que $\operatorname{NWM}(C(K))$ é espaçável. No caso em que $C(K)$ contém $c_{0}$ como quociente, os resultados do Capítulo 4 foram mais longe no sentido que foi provando que $\operatorname{NWM}(C(K)) \cup\{0\}$ contém um subespaço de $\mathcal{L}(C(K)))$ isomorfo a $\ell_{\infty}$ e, consequentemente, com densidade não enumerável, enquanto que o subespaço de $\operatorname{NWM}(C(K)) \cup\{0\}$ construído em [FKP13] é separável.

Assim, para o caso que $C(K)$ contém $c_{0}$ como quociente (em particular no caso que contem $c_{0}$ complementado) os resultados do Capítulo 4 demostraram que NWMC $(K)$ é c-espaçável, melhorando o resultado de Fajardo et al. para esses casos.

Fajardo, em [Faj12], assumindo Axioma $\diamond$, construiu um exemplo de um espaço $C(K) \operatorname{com} 2^{\omega}$ quocientes indecomponíveis. Das propriedades desse espaço se deduz que $K$ não contém $\beta \mathbb{N}$ como subespaço. Adicionalmente, nesse mesmo trabalho, é apresentado um exemplo, assumindo $\mathrm{CH}$, de um espaço fracamente Koszmider que contém $\beta \mathbb{N}$ como subespaço. No Capítulo 6 é construído um exemplo de um espaço $C(K)$ que tem $2^{\omega}$ quocientes indecomponíveis e $K$ contém $\beta \mathbb{N}$ como subespaço. O Axioma $\diamond$ é assumido na nossa construção.

As construções dos Capítulos 6 e 7 usam as extensões de espaços compactos por sequências de funções contínuas, duas a duas disjuntas como uma das ferramentas principais. Dados um compacto e uma sequência de funções reais, a extensão de $K$ por essa sequência nem sempre é forte ou completa. Trocar a sequência por uma subsequência, ou por outras funções com suportes contidos nos das que foram originalmente dadas pode ser suficiente para alguns objetivos específicos, como nalgumas construções de espaços fracamente Koszmider. No Capítulo 5 se estudaram extensões por uma sequência de funções contínuas, no caso que existir um homeomorfismo entre dois subconjuntos de $K$ disjuntos que eram fechos de abertos e que as funções respeitavam esse homeomorfismo (i.e. se $\varphi$ é o homeomorfismo então $\left.f_{n} \circ \varphi(x)=f_{n}(x)\right)$. 


\subsection{Comentários finais e problemas em aberto}

Sabe-se, por Koszmider (ver Lema 2.9), que nos espaços conexos compactos $K$ que não contêm abertos-borboleta os subespaços $K \backslash\{x\}$ são $C^{*}$-imersos em $K$. Não sabemos se todo espaço de Koszmider não contém abertos-borboleta. É conhecido que um subespaço $E$ de um espaço topológico $Y$ é $C^{*}$-imerso em $Y$ se, e somente se, quaisquer dois subconjuntos de $E$ disjuntos e funcionalmente fechados (i.e. pré-imagens de 0 por alguma função contínua com imagem em [0,1]) são completamente separados em $X$ (veja o Teorema 3.3 de [Kar15]).

Como foi mencionado antes, existem espaços de Banach $C(K)$ indecomponíveis tais que $K$ não são Koszmider. Mas permanece desconhecido se para todo espaço $C(K)$ indecomponível o compacto $K$ é fracamente Koszmider. Também não sabemos se o Corolário 7.3 acontece em ZFC.

Um problema que abordamos foi se existe em $\mathrm{ZFC}$ ou em $\mathrm{ZFC}+\mathrm{CH}$ um espaço fracamente Koszmider $K$ tal que $C(K)$ tem $2^{\omega}$ quocientes indecomponíveis. O exemplo devido a Fajardo assume o Axioma $\diamond$. Se o Teorema 7.1 acontece em ZFC+CH então o subespaço $L=\overline{K_{[0,2 / 3[}}$ do $K$ tem a propriedade que $C(L)$ tem $2^{\omega}$ quocientes indecomponíveis. Mas uma das limitações para retirar o axioma $\diamond$ quando se usam construções como a do Capítulo 7 é enumerar as medidas de Radon em $[0,1]^{\omega_{1}}$ de maneira que possam ser ser determinas por medidas definidas sobre os compactos construídos ao longo da indução.

$\mathrm{Na}$ introdução foram comentadas as relações entre as condições (A)-(E) relativas aos espaços com poucos operadores. Vimos que a condição (A) (i.e. $K$ Koszmider) implica (B) (i.e. $K$ fracamente Koszmider), e que (B) implica (C) (i.e. $C(K)$ não é isomorfo a seus hiperplanos) mas (B) não implica (A). No caso que $K$ é conexo, (A) implica (D) $(C(K)$ indecomponível). Por outro lado, se $K$ é infinito e totalmente desconexo, $C(K)$ não pode ser indecomponível. Com efeito, se $K$ tem apenas um ponto limite, então $C(K)$ é isomorfo a $c_{0}(I)$, para algum conjunto infinito $I$, o qual pode ser decomposto como $c_{0}(I) \oplus c_{0}(I)$. Se $K$ tem pelo menos dois pontos limites, pelo fato que $K$ é totalmente desconexo, é possível achar dois subconjuntos abertos-fechados disjuntos $A$ e $B$ de $K$ tais que $A \cup B=K$. Neste caso, $C(K) \approx C(A) \oplus C(B)$. Assim (D) implica (E) ( Se $L$ é totalmente desconexo, $C(K)$ não é isomorfo a $C(L))$.

Em [AK13] foi provado que (E) não implica nenhuma das condições (A) até (D). Dos resultados em [Sch08a] (ver Teoremas 3.4 e 3.5) se deduz que (D) não implica (A). No Capítulo 3 foi provado que (B) não implica (D). O seguinte diagrama apresenta um esboço das relações entre as propriedades (A) até (E) no caso $K$ conexo. As setas $\rightarrow$ e $\nrightarrow$ devem ser lidas como "implica" e "não implica", respectivamente.

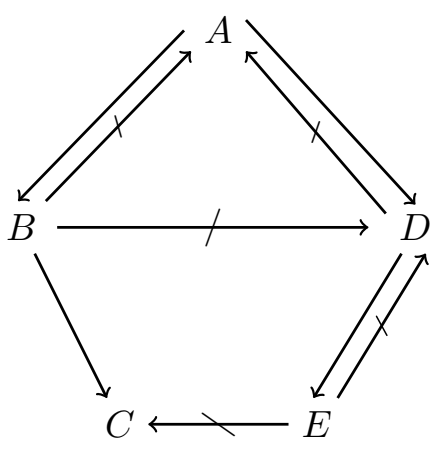

O espaço construído no Capítulo 7 vai na direção de responder (pelo menos parcialmente) à 
pergunta se (D) implica (B). Para completar o diagrama, resta resolver as seguintes perguntas, que permanecem em aberto.

P.1. Existe um espaço de Banach indecomponível $C(K)$ tal que $K$ não é fracamente Koszmider? Ver Fajardo, et al., [FGP19].

P.2. Existe um espaço de Banach indecomponível $C(K)$ isomorfo a seus hiperplanos? Ver Fajardo, et al., [FGP19].

P.3. Existe um espaço $K$ não fracamente Koszmider tal que $C(K)$ não é isomorfo a seus hiperplanos? Ver Fajardo, et al., [FGP19].

P.4. Existe um espaço conexo $K$ não fracamente Koszmider tal que $C(K)$ é isomorfo a $C(L)$, para algum $L$ compacto totalmente desconexo? Ver Koszmider, [Kos10].

P.5. Existe um espaço conexo $K$ tal que $C(K)$ não é isomorfo a seus hiperplanos mas $C(K)$ é isomorfo a $C(L)$, para algum $L$ totalmente desconexo? Ver Fajardo, et al., [FGP19]. 


\section{Referências Bibliográficas}

[AB77] D. Alspach e Y. Benyamini. Primariness of spaces of continuous functions on ordinals. Israel Journal of Mathematics, 27(1):64-92, 1977. 35

[AGS05] R. Aron, V.I. Gurariy e J. Seoane. Lineability and spaceability of sets of functions on $\mathbb{R}$. Proceedings of the American Mathematical Society, 133(3):795 - 803, 2005. 37

[AH11] S. A. Argyros e R. G. Haydon. A hereditarily indecomposable $\mathcal{L}_{\infty}$-space. Acta Mathematica, 206(1):1-54, 2011. 1

[AK06] F. Albiac e N. J. Kalton. Topics in Banach space theory, volume 233. Springer, 2006. 12, $33,35,41$

[AK13] A. Avilés e P. Koszmider. A continuous image of a Radon-Nikodým compact space which is not Radon-Nikodým. Duke Math. J., 162(12):2285-2299, 2013. 3, 89

[BD02] S. Barov e J. J. Dijkstra. On boundary avoiding selections and some extension theorems. Pacific journal of mathematics, 203(1):79-87, 2002. 25, 87

[BF17] A. S. V. Barbeiro e R. A. S. Fajardo. Suprema of continuous functions on connected spaces. São Paulo Journal of Mathematical Sciences, 11(1):189-199, 2017. 45

[BK14] C. Brech e P. Koszmider. $\ell_{\infty}-$ sums and the Banach space $\ell_{\infty} / c_{0}$. Fundamenta Mathematicae, 224:1755-185, 2014. 35

[Cem84] P. Cembranos. $C(K, E)$ contains a complemented copy of $c_{0}$. Proceedings of the American Mathematical Society, 91(4):556-558, 1984. 41

[DR91] L. Drewnowski e J. W. Roberts. On the primariness of the Banach space $\ell_{\infty} / c_{0}$. Proceedings of the American Mathematical Society, 112(4):949-957, 1991. 35

[DS58] N. Dunford e J. T. Schwartz. Linear Operators I. Interscience Piblishers, New York London, 1958. 11

[DT13] R. R. Dias e F. D. Tall. Indestructibility of compact spaces. Topology and its Applications, 160(18):2411-2426, 2013. Special Issue: Fourth Workshop on Coverings, Selections and Games in Topology. 67

[DU77] J. Diestel e J. J. Uhl. Vector measures. math. surveys, 15. Amer. Math. Soc., Providence, Rhode Island, 1977. 11, 12, 33

[Eng89] R. Engelking. General topology. Sigma Series in Pure Mathematics, 6, Heldermann Verlag, Berlin, 2 edição, 1989. 7, 8, 70

[Faj07] R. A. S. Fajardo. Construções consistentes de espaços de Banach $C(K)$ com poucos operadores. Tese de Doutorado, Instituto de Matemática e Estatística, Universidade de São Paulo, Brasil, Setembro 2007. 3, 14

[Faj09] R. A. S. Fajardo. An indecomposable Banach space of continuous functions which has small density. Fundamenta Mathematicae, 202(1):43-63, 2009. 3, 31 
[Faj10] R. A. S. Fajardo. Essentially incomparable Banach spaces of continuous functions. Bulletin of the Polish Academy of Sciences. Mathematics, 3(58):247-258, 2010. 4, 25, 34, 88

[Faj12] R. A. S. Fajardo. Quotients of indecomposable Banach spaces of continuous functions. Studia Mathematica, 3(212):259-283, 2012. 4, 5, 6, 15, 17, 27, 44, 46, 48, 53, 57, 61, 63, $66,67,68,88$

[FGP19] R. A. S. Fajardo, A. Gómez e L. Pellegrini. Decompositions of Banach spaces $C(K)$ with few operators. São Paulo Journal of Mathematical Sciences, 13(1):305-319, 2019. 6, 41, 90

[FKP13] R. A. S. Fajardo, P. L. Kaufmann e L. Pellegrini. Spaceability in sets of operators on $C(K)$. Topology and its Applications, 160(2):387 - 393, 2013. 5, 23, 37, 38, 88

[Fra95] M. Frantz. Controlling Tietze-Urysohn extensions. Pacific Journal of Mathematics, 169(1):53-73, 1995. 24, 25

[GM93] W. T. Gowers e B. Maurey. The unconditional basic sequence problem. Journal of the American Mathematical Society, 6(4):851-874, 1993. 2, 3

[GQ04] V. I. Gurariy e L. Quarta. On lineability of sets of continuous functions. Journal of Mathematical Analysis and Applications, 294(1):62 - 72, 2004. 37

[Jec03] T. Jech. Set Theory. Springer Monographs in Mathematics (Springer-Verlag, Berlin), the third millennium edition, revised and expanded edição, 2003. 9, 15, 66

[JP09] J. F. Mena Jurado e A. Rodríguez Palacios. Weakly compact operators on non-complete normed spaces. Expositiones Mathematicae, 27(2):143 - 151, 2009. 38

[Kar15] O. Karlova. On C-embedded subspaces of the Sorgenfrey plane. Applied General Topology, 16(1):65-74, 2015. 89

[Kos04] P. Koszmider. Banach spaces of continuous functions with few operators. Mathematische Annalen, 330(1):151-183, 2004. 2, 3, 4, 5, 17, 18, 19, 20, 22, 27, 31, 32, 33, 35, 43, 44, 46, $47,53,65,67,68,75,76,87$

[Kos05] P. Koszmider. On decompositions of Banach spaces of continuous functions on Mrówka's spaces. Proceedings of the American Mathematical Society, 133(7):2137-2146, 2005. 33

[Kos10] P. Koszmider. A survey on Banach spaces $C(K)$ with few operators. Revista de la Real Academia de Ciencias Exactas, Fisicas y Naturales. Serie A. Matematicas, 104(2):309326, 2010. 18, 90

[Kun80] K. Kunen. Set theory. An introduction to independence proofs. North Holland, 1980. 9, 15,66

[Lin75] J. Lindenstrauss. Some open problems in Banach space theory. Séminaire Choquet. Initiation à l'analyse, 15:1-9, 1975. 1

[LP71] J. Lindenstrauss e A. Pełczyński. Contributions to the theory of the classical Banach spaces. Journal of Functional Analysis, 8(2):225 - 249, 1971. 35

[Mib] Y. Mibu. On Baire functions on infinite product spaces. Proceedings of the Imperial Academy. 65

[Muj97] J. Mujica. Separable quotiens of Banach spaces. Revista Matematica de la universidad Complutence de Madrid, 10(2):299-330, 1997. 4, 67 
[Peł60] A. Pełczyński. Projections in certain Banach spaces. Studia Mathematica, 2(19):209-228, 1960. 11

[Ple04] G. Plebanek. A construction of a Banach space $C(K)$ with few operators. Topology and its Applications, 143(1-3):217-239, 2004. 3, 19, 22, 32, 75

[PS59] A. Pełczyński e Z. Semadeni. Spaces of continuous functions (iii) (spaces $C(\Omega)$ for $\Omega$ without perfect subsets). Studia Mathematica, 18(2):211-222, 1959. 67

[Sch82] W. Schachermayer. On some classical measure-theoretic theorems for non-sigmacomplete Boolean algebras. Dissertationes Math. (Rozprawy Mat.), 214, 1982. 33, 41

[Sch08a] I. Schlackow. Centripetal operators and Koszmider spaces. Topology and its Applications, 155(11):1227-1236, 2008. 3, 4, 19, 22, 31, 32, 34, 87, 88, 89

[Sch08b] I. Schlackow. Classes of $C(K)$ spaces with few operators. Tese de Doutorado, Oxford University, UK, 2008. 22

[Sem71] Z. Semadeni. Banach spaces of continous functions (monografie matematyczne 55). PWN--Polish Scientific Publ., Warszawa, 1971. 67

[SS88] S. Shelah e J. Steprāns. A Banach space on which there are few operators. Proceedings of the American Mathematical Society, 104(1):101-105, 1988. 2, 33 


\section{Índice Remissivo}

$C^{*}$-imerso, 20

$\lambda$-espaçável, 37

$\lambda$-lineável, 37

c-espaçável, 38, 41

abertos borboleta, 20

abertos-borboleta, 68

axioma

$$
\text { Diamante, } 67
$$

conjunto

dos operadores não multiplicadores fracos, 37,41

raro, 47,68

densidade, 37

Dunford-Pettis, 11

espaçável, 37, 38

espaço

$C(K)$

indecomponível, 31

tendo quociente isomorfo a $c_{0}, 38$

com poucos operadores

da forma $C(K), 19,31$

no sentido Argyros e Haydon, 17

no sentido Gowers e Maurey, 17

com sequências convergentes, 12

de Banach

$$
\text { here }
$$

indecomponível, 1

primário, 35

de Grothendieck, 33

de Koszmider , 19

disperso, 17, 67

fortemente rígido, 22

fracamente Koszmider, 20, 22, 25, 32

frcamente Koszmider

sem pontos isolados, 23

Koszmider, 68, 75

sem sequencias convergentes, 23

totalmente desconexo, 89 extensão de $K$ por uma sequência de funções contínuas, 44

completa, 44, 61, 64

forte, 44,45

funções

disjuntas, 18

König, 66

lineável, 37

medida de Radon, 19

medida regular, 25

Mibu, 65

operador

estritamente singular, 12

fracamente compacto, 12, 38

multiplicação fraca, 18

multiplicador fraco, 18

quocientes indecomponíveis, 75

Riesz, 15

Rosenthal, 11

Schachermayer, 33

sistema inverso, 7

limite do, 7,8

l, Sobczyk, 10

subespaço

complementado de $C(K), 32$ 